MÉMOIRES DE LA SMF 93

\title{
PROPRIÉTÉS DE L'INTÉGRALE DE CAUCHY HARISH-CHANDRA POUR CERTAINES PAIRES DUALES D'ALGÈBRES DE LIE
}

Florent Bernon

Société Mathématique de France 2003

Publié avec le concours du Centre National de la Recherche Scientifique 


\section{F. Bernon}

Laboratoire analyse, géométrie et applications, UMR CNRS 7539,

Département de mathématiques, Université Paris 13, 93430 Villetaneuse, France.

E-mail : bernon@math.univ-paris13.fr

Mathematisches Institut, Georg-August Universität Göttingen, Bunsenstrasse 3-5, D-37073 Göttingen, Allemagne.

E-mail : bernon@uni-math.gwdg.de 


\title{
PROPRIÉTÉS DE L'INTÉGRALE DE CAUCHY HARISH-CHANDRA POUR CERTAINES PAIRES DUALES D'ALGĖBRES DE LIE
}

\author{
Florent Bernon
}

\begin{abstract}
Résumé. - On considère un groupe symplectique $S p$ et une paire duale réductive et irréductible $\left(G, G^{\prime}\right)$ de $S p$ au sens de R. Howe. On désigne par $\mathfrak{g}$ (resp. $\left.\mathfrak{g}^{\prime}\right)$ les algèbres de Lie de $G$ (resp. $G^{\prime}$ ). T. Przebinda définit une application appelée intégrale de Cauchy Harish-Chandra et notée Chc qui associe à toute fonction de $\mathcal{D}(\mathfrak{g})$ une fonction définie sur $\mathfrak{g}^{\text {/reg }}$, l'ouvert des éléments semi-simples réguliers. Dans cet article, on montre que ces fonctions sont des intégrales invariantes si la paire est de type II et possèdent les propriétés locales des intégrales invariantes si la paire est formée de groupes unitaires de même rang. Les relations de saut sont alors obtenues à une constante multiplicative près.
\end{abstract}

\section{Abstract (Properties of the Cauchy Harish-Chandra integral for some dual pairs of Lie algebras)}

We consider a symplectic group $S p$ and an irreductible dual pair $\left(G, G^{\prime}\right)$ in $S p$ in the sense of R. Howe. Let $\mathfrak{g}$ (resp. $\mathfrak{g}^{\prime}$ ) be the Lie algebra of $G$ (resp. $G^{\prime}$ ). T. Przebinda has defined a map Chc, called the Cauchy Harish-Chandra integral from the space of smooth compactly supported functions of $\mathfrak{g}$ to the space of functions defined on the open set $\mathfrak{g}^{\text {reg }}$ of semisimple regular elements of $\mathfrak{g}^{\prime}$. We prove that these functions are invariant integrals if $G$ and $G^{\prime}$ are linear groups and behave locally like invariant integrals if $G$ and $G^{\prime}$ are unitary groups of same rank. In this last case, we obtain the jump relations up to a multiplicative constant which only depends on the dual pair. 



\section{TABLE DES MATIÈRES}

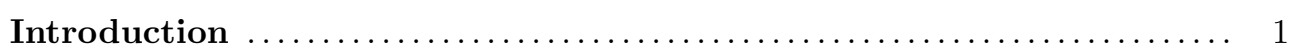

1. Propriétés de Chc pour les paires de type II $\ldots \ldots \ldots \ldots \ldots \ldots \ldots \ldots$

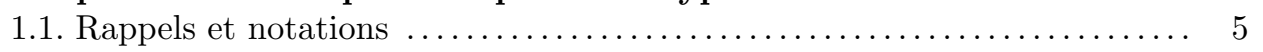

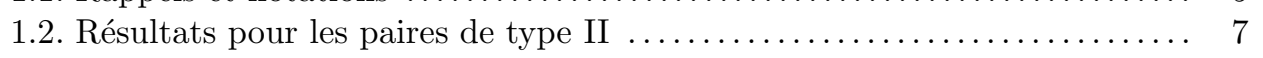

2. Définition de Chc pour les paires duales unitaires $\ldots \ldots \ldots \ldots \ldots \ldots .9$

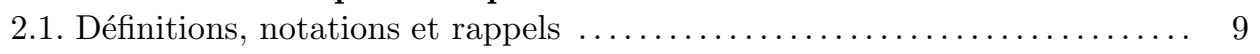

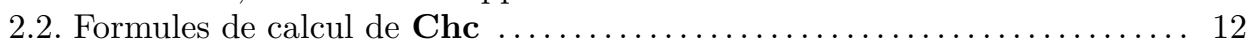

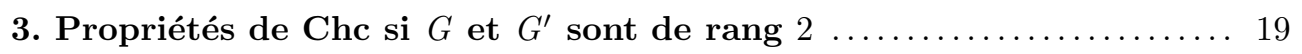

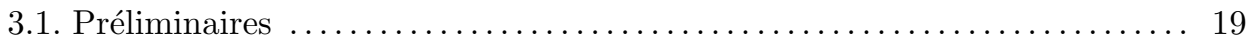

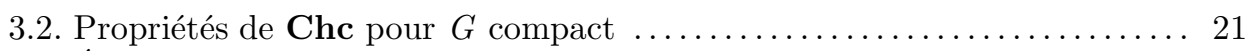

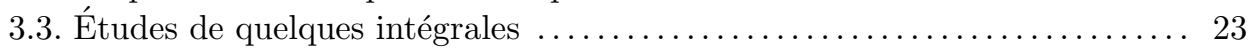

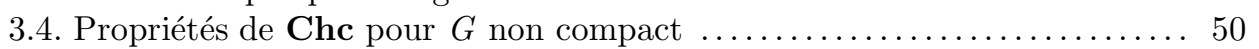

3.5. Propriétés de Chc pour $G$ compact ou non $\ldots \ldots \ldots \ldots \ldots \ldots \ldots \ldots \ldots$

4. Propriétés des intégrales invariantes $\ldots \ldots \ldots \ldots \ldots \ldots \ldots \ldots \ldots \ldots$

4.1. L'intégrale invariante de Harish-Chandra $\ldots \ldots \ldots \ldots \ldots \ldots \ldots \ldots \ldots \ldots$

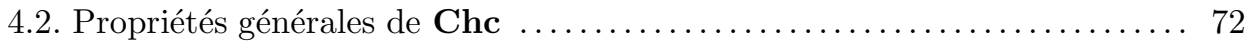

4.3. Décomposition des intégrales invariantes $\ldots \ldots \ldots \ldots \ldots \ldots \ldots \ldots \ldots \ldots$

4.4. Décomposition en sous-espaces élémentaires $\ldots \ldots \ldots \ldots \ldots \ldots \ldots \ldots \ldots$

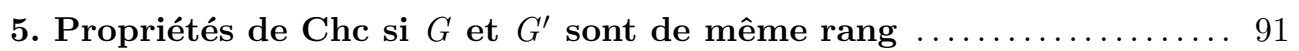

5.1. Pour une sous-algèbre de Cartan compacte . . . . . . . . . . . . . . . . . 91

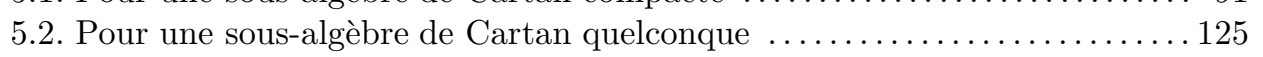

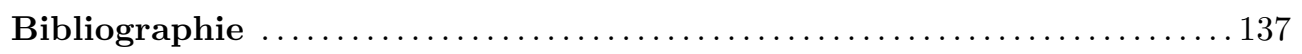





\section{INTRODUCTION}

Soit $W$ un $\mathbb{R}$-espace vectoriel de dimension finie muni d'un produit symplectique $\langle$,$\rangle . On note S p(W)$ et $\mathfrak{s p}(W)$ le groupe et l'algèbre de Lie associés. Soit $\left(G, G^{\prime}\right)$ une paire duale irréductible de $S p(W)$ au sens de Howe [4]. Pour un sous-groupe de Cartan $H^{\prime}$ de $G^{\prime}$, on note $T^{\prime}$ (resp. $A^{\prime}$ ) sa partie compacte (resp. sa partie déployée) c'est à dire les éléments de $H^{\prime}$ qui ont leurs valeurs propres de module 1 (resp. réelles positives). Soient $A^{\prime \prime}=S p(W)^{A^{\prime}}$ le centralisateur de $A^{\prime}$ dans $S p(W)$ et $A^{\prime \prime \prime}$ le centralisateur de $A^{\prime \prime}$ dans $S p(W)$. Il existe un ouvert dense de $W$ que l'on note $W_{A^{\prime \prime \prime}}$ tel que $A^{\prime \prime \prime} \backslash W_{A^{\prime \prime \prime}}$ soit une variété d'après [6, p.302]. On considère une mesure $d \dot{w}$ sur $A^{\prime \prime \prime} \backslash W_{A^{\prime \prime \prime}}$. Pour un sous-espace $u$ de $\mathfrak{s p}(W)$, on considère l'application moment $\tau_{u}: W \rightarrow u^{*}$ (le dual de $u$ ) telle que $\tau_{u}(w)(x)=\langle x w, w\rangle$ pour $x \in u$ et $w \in W$. Pour $x \in \mathbb{R}$, on pose $\chi(x)=e^{2 \mathrm{i} \pi x}$ et pour $w \in W$

$$
\chi_{x}(w)=\chi\left(\frac{1}{4} \tau_{\mathfrak{s p}(W)}(w)(x)\right) .
$$

On désigne par $\mathfrak{a}^{\prime \prime}$ l'algèbre de Lie de $A^{\prime \prime}$. Pour tout $\psi \in \mathcal{D}\left(\mathfrak{a}^{\prime \prime}\right)$ (l'espace des fonctions lisses à support compact), Przebinda $([\mathbf{6}])$ montre que

$$
\int_{A^{\prime \prime \prime} \backslash W_{A^{\prime \prime \prime}}}\left|\int_{\mathfrak{a}^{\prime \prime}} \psi(x) \chi_{x}(w) d x\right| d \dot{w}<\infty .
$$

On considère la distribution sur $\mathfrak{a}^{\prime \prime}$

$$
C h c(\psi)=\int_{A^{\prime \prime \prime} \backslash W_{A^{\prime \prime \prime}}} \int_{\mathfrak{a}^{\prime \prime}} \psi(x) \chi_{x}(w) d x d \dot{w} .
$$

Pour $x^{\prime} \in \mathfrak{h}^{\prime}$ semi-simple régulier, l'injection $i_{x^{\prime}}: \mathfrak{g} \rightarrow \mathfrak{a}^{\prime \prime}, x \mapsto x+x^{\prime}$, est transverse au front d'onde $W F(C h c)$ de $C h c$.

Cela permet de définir l'image réciproque de $C h c$ par l'injection $i_{x^{\prime}}$ que l'on note $C h c_{x^{\prime}}$. Pour $\phi \in \mathcal{D}(\mathfrak{g})$, on note $\operatorname{Chc}(\phi)$ la fonction définie sur $\mathfrak{g}^{\text {'reg }}$ par

$$
\operatorname{Chc}(\phi)\left(x^{\prime}\right)=C h c_{x^{\prime}}(\phi)
$$


Dans le cas où $\left(G, G^{\prime}\right)$ est une paire dite «stable range », pour toute orbite nilpotente $\mathcal{O}^{\prime}$ de $\mathfrak{g}^{\prime *}$, il existe une unique orbite nilpotente $\mathcal{O}$ de $\mathfrak{g}^{*}$ dense dans $\tau_{\mathfrak{g}} \circ \tau_{\mathfrak{g}^{\prime}}{ }^{-1}\left(\mathcal{O}^{\prime}\right)$. On note $\mu(\mathcal{O})$ et $\mu\left(\mathcal{O}^{\prime}\right)$ les mesures positives sur $\mathcal{O}$ et $\mathcal{O}^{\prime}$ respectivement $G$ et $G^{\prime}$ invariantes. D'après le théorème 1.19 de $[6]$ à une constante multiplicative près, on a

$$
\widehat{\mu}_{\mathcal{O}}(\phi)=\sum \frac{1}{\left|W\left(H^{\prime}\right)\right|} \int_{\mathfrak{h}^{\prime}} \widehat{\mu}_{\mathcal{O}^{\prime}}\left(x^{\prime}\right) D_{\mathfrak{g}^{\prime}}\left(x^{\prime}\right)^{2} \mathbf{C h c}(\phi)\left(x^{\prime}\right) d x^{\prime}
$$

pour $\phi \in \mathcal{D}(\mathfrak{g})$ où la somme s'effectue sur un système de représentants de sous-algèbres de Cartan de $\mathfrak{g}$ et $D_{\mathfrak{g}^{\prime}}\left(x^{\prime}\right)=\mid \operatorname{det}\left(\left.a d\left(x^{\prime}\right)_{\mathfrak{g}^{\prime} / \mathfrak{h}^{\prime}}\right|^{1 / 2}\right.$ pour $x^{\prime} \in \mathfrak{h}^{\text {reg }}$. Cette égalité suggère que $\mathbf{C h c}(\phi)$ a les propriétés locales d'une intégrale orbitale.

L'objet de cet article est de montrer que pour les paires duales irréductibles de type I formées de groupes unitaires de même rang, la fonction $\mathbf{C h c}(\phi)(\phi \in \mathcal{D}(\mathfrak{g}))$ qui est définie sur $\mathfrak{g}^{\text {/reg }}$ possède les propriétés locales des intégrales orbitales de $\mathfrak{g}^{\prime}$. Plus précisément, on montre que la fonction $\mathbf{C h c}(\phi)$ est lisse sur $\mathfrak{g}^{\prime \text { reg }}$ et $G^{\prime}$-invariante. On exhibe une famille de sous-algèbres de Cartan $\left(\mathfrak{h}_{N}\right)_{N \in \Omega^{\prime}}$ de $\mathfrak{g}^{\prime}$ indexée sur un ensemble $\Omega^{\prime}$ qui représente l'ensemble des systèmes de racines admissibles d'un système de racines positives d'une sous-algèbre de Cartan compacte de $\mathfrak{g}^{\prime}$. Pour chaque $N \in \Omega^{\prime}$, on construit une fonction $\operatorname{Chc}(\phi)_{N}$ sur $\mathfrak{h}_{N}^{\text {reg }}$.

Puis, on montre que toutes les dérivées de $\mathbf{C h c}(\phi)_{N}$ sont localement bornées et se prolongent par continuité sur l'adhérence des composantes connexes de l'ensemble des éléments de $\mathfrak{h}_{N}^{\prime}$ qui ne s'annulent pour aucune racine imaginaire non compacte de $\mathfrak{h}_{N}^{\prime}$. Pour une racine $\alpha$ imaginaire non compacte, on note $\left\langle\partial(w) \operatorname{Chc}(\phi)_{N}\right\rangle$ la fonction saut qui est définie d'après ce qui précède. On montre enfin que pour deux sous-algèbres de Cartan $\mathfrak{h}_{N}^{\prime}$ et $\mathfrak{h}_{M}^{\prime}$ (avec $N, M \in \Omega^{\prime}$ ) successives dans l'ordre de Hiraï, il existe une constante $C$ telle que pour tout élément $w$ de l'algèbre symétrique de $\mathfrak{h}_{N}^{\prime}$, on a

$$
\left\langle\partial(w) \mathbf{C h c}(\phi)_{N}\right\rangle=\mathrm{i} C \partial\left(w^{\prime}\right) \mathbf{C h c}(\phi)_{M}
$$

où $w^{\prime}$ est l'image de $w$ par une transformée de Cayley.

Le plan de cet article est le suivant :

Dans le chapitre 1, en utilisant une expression obtenue par T. Przebinda dans [6] de Chc pour les paires de type II, on montre qu'il existe une fonction $\psi \in \mathcal{D}\left(\mathfrak{g}^{\prime}\right)$ telle que $\mathbf{C h c}(\phi)$ représente l'intégrale orbitale de $\psi$, Théorème 1.2.2.

Dans le chapitre 2, on introduit les notations nécessaires à la définition de $\mathbf{C h c}$ pour les paires de groupes unitaires et on précise les expressions de $\mathbf{C h c}(\phi)$ obtenues par T. Przebinda dans [6] pour une sous-algèbre de Cartan compacte de $\mathfrak{g}^{\prime}$, Théorème 2.2.4 puis pour une sous-algèbre de Cartan quelconque de $\mathfrak{g}^{\prime}$, Théorème 2.2.7. Cette dernière expression est définie modulo une constante qui détermine $C$.

Dans le chapitre 3 , on considère les paires $\left(\mathfrak{g}, \mathfrak{g}^{\prime}\right)$ avec $\mathfrak{g}$ et $\mathfrak{g}^{\prime}$ de rang 2 . Après avoir rappelé les propriétés des intégrales invariantes de Harish-Chandra sur $\mathfrak{u}(2)$ et $\mathfrak{u}(1,1)$, on obtient les propriétés de Chc aisément si $\mathfrak{g}$ est compacte (i.e. $\mathfrak{u}(2))$ dans la section 1. Dans la section 2, on étudie le développement asymptotique de certaines 
fonctions qui permettent dans la section 3 d'obtenir les propriétés locales de Chc si $\mathfrak{g}$ est non compacte (i.e. $\mathfrak{u}(1,1))$. Dans la section 4 , on rassemble les résultats obtenus pour $\left(\mathfrak{g}, \mathfrak{g}^{\prime}\right)$ avec $\operatorname{rang}(\mathfrak{g})=\operatorname{rang}\left(\mathfrak{g}^{\prime}\right)=2$, Théorème 3.5.1.

Les formules obtenues par T. Przebinda rappelées dans le chapitre 2 montrent que $\operatorname{Chc}(\phi)$ ne dépend que de l'intégrale orbitale de $\phi$. Pour généraliser aux paires de rang quelconque, on montre dans la section 3 du chapitre 4 que les intégrales orbitales de $\mathfrak{g}$ (avec $\operatorname{rang}(\mathfrak{g}) \geqslant 2$ ) peuvent être approchées par des sommes de produits d'intégrales orbitales de sous-groupes réductifs de $G$ de rang $\leqslant 2$, Théorèmes 4.3.11 et 4.4.4. Ces propriétés permettent d'utiliser les résultats du chapitre 3 pour généraliser aux paires $\left(\mathfrak{g}, \mathfrak{g}^{\prime}\right)$ de rang supérieur. Le principe de cette réduction est le suivant : Désignons l'espace des intégrales orbitales de $\mathfrak{g}$ par $\mathcal{E}$, alors on construit un espace topologique $\widetilde{\mathcal{E}}$ contenant $\mathcal{E}$ tel que $\mathbf{C h c}$ se prolonge naturellement à $\widetilde{\mathcal{E}}$. Les résultats de décomposition mentionnés précédemment exhibent une partie $\mathcal{E}^{\prime}$ de $\widetilde{\mathcal{E}}$ telle que tout élément de $\mathcal{E}$ est dans l'adhérence de $\mathcal{E}^{\prime}$. Dans le chapitre suivant, on utilise le fait que le Chc d'un élément de $\mathcal{E}^{\prime}$ s'exprime simplement à partir du Chc pour des paires de rang $\leqslant 2$.

Dans le chapitre 5 , dans un premier temps, on étudie les propriétés de $\mathbf{C h c}(\phi)$ sur la sous-algèbre de Cartan compacte $\mathfrak{h}_{\varnothing}^{\prime}$. On montre que toutes les dérivées de Chc $(\phi)_{\varnothing}$ se prolongent par continuité sur l'adhérence des composantes connexes de $\mathfrak{h}_{\varnothing}^{\text {reg }}$, Théorème 5.1.25 puis on calcule le saut de cette fonction par rapport aux hyperplans associés aux racines (imaginaires) de $\mathfrak{h}_{\varnothing}^{\prime}$. La fonction saut obtenue est reliée au Chc pour une paires $\left(\mathfrak{g}_{1}, \mathfrak{g}_{1}^{\prime}\right)$ telle que $\operatorname{rang}\left(\mathfrak{g}_{1}\right)=\operatorname{rang}\left(\mathfrak{g}_{1}^{\prime}\right)=\operatorname{rang}(\mathfrak{g})-2$, Théorème 5.1.30. Dans la deuxième section, on obtient les propriétés locales de $\mathbf{C h c}(\phi)$ pour une sous-algèbre de Cartan quelconque de $\mathfrak{g}^{\prime}$, Théorème 5.2.4 et on calcule le 'saut' de cette fonction, Théorème 5.2.5.

Ce travail a fait l'objet d'une note [1] .

Remerciements. - Je tiens à remercier le Professeur A. Bouaziz pour de très utiles conversations pendant l'élaboration de ce travail. Je remercie également le Professeur T. Przebinda pour son aide. 



\section{CHAPITRE 1}

\section{PROPRIÉTÉS DE Chc POUR LES PAIRES DE TYPE II}

\subsection{Rappels et notations}

DÉFInITION 1.1.1. — Les paires de type II sont les paires

$$
\left(G L(\mathrm{n}, \mathbb{R}), G L\left(\mathrm{n}^{\prime}, \mathbb{R}\right)\right),\left(G L(\mathrm{n}, \mathbb{C}), G L\left(\mathrm{n}^{\prime}, \mathbb{C}\right)\right) \text { et }\left(G L(\mathrm{n}, \mathbb{H}), G L\left(\mathrm{n}^{\prime}, \mathbb{H}\right)\right)
$$

avec $n, n^{\prime} \in \mathbb{N}$.

On note $\mathbb{D}$ l'un des corps $\mathbb{R}, \mathbb{C}$ ou $\mathbb{H} ; V$ (resp. $\left.V^{\prime}\right)$ un $\mathbb{D}$-espace vectoriel de dimension $\mathrm{n}\left(\right.$ resp. $\left.\mathrm{n}^{\prime}\right)$ avec $\mathrm{n}^{\prime} \leqslant \mathrm{n}$. On peut supposer que $V^{\prime}$ est un sous-espace de $V$ et on fixe un supplémentaire $U$ de $V^{\prime}$ dans $V$. On note $G=G L(V), G^{\prime}=G L\left(V^{\prime}\right)$ et $\mathfrak{g}, \mathfrak{g}^{\prime}$ les algèbres de Lie de $G$ et $G^{\prime}$. On considère les sous-algèbres de $\mathfrak{g}$ suivantes :

$$
\begin{gathered}
\mathfrak{p}=\left\{x \in \mathfrak{g} \mid x\left(V^{\prime}\right) \subset V^{\prime}\right\}, \\
\mathfrak{n}=\operatorname{Hom}\left(U, V^{\prime}\right) \text { et } \mathfrak{l}=\mathfrak{g}^{\prime} \oplus \mathfrak{g l}(U) .
\end{gathered}
$$

La sous-algèbre $\mathfrak{p}$ est une sous-algèbre parabolique de $\mathfrak{g}$ de radical nilpotent $\mathfrak{n}$ et $\mathfrak{l}$ est un facteur de Levi de $\mathfrak{p}$. On note $\operatorname{Car}(\mathfrak{g})$ (resp. $\left.\operatorname{Car}\left(\mathfrak{g}^{\prime}\right)\right)$ l'ensemble des sous-algèbres de Cartan de $\mathfrak{g}$. Pour $\mathfrak{h} \in \operatorname{Car}(\mathfrak{g})\left(\operatorname{resp} . \mathfrak{h}^{\prime} \in \operatorname{Car}\left(\mathfrak{g}^{\prime}\right)\right)$, on note $H$ (resp. $\left.H^{\prime}\right)$ le sousgroupe de Cartan associé de $G$ (resp. $\left.G^{\prime}\right)$. On désigne par $K$ un sous-groupe compact maximal de $G$. On fixe une forme $G$-invariante $\kappa_{\mathfrak{g}}$ non dégénérée sur $\mathfrak{g}$ à laquelle on associe une mesure de Haar $d g$ sur $G$. La forme $\kappa_{\mathfrak{g}}$ se restreint sur $\mathfrak{h}$ avec $\mathfrak{h} \in \operatorname{Car}(\mathfrak{g})$ en une forme non dégénérée à laquelle on associe une mesure $d h$ sur $H$. Sur l'espace quotient $G / H$, on considère la mesure quotient $d g / d h$ habituelle. Sur $\mathfrak{g}^{\prime}$, on procède de la même manière avec une forme $\kappa_{\mathfrak{g}^{\prime}} G^{\prime}$-invariante non dégénérée. On note $\mathfrak{g}^{\text {reg }}$ l'ensemble des éléments semi-simples réguliers de $\mathfrak{g}$ et pour un sous-espace vectoriel $u$ de $\mathfrak{g}$, on note $u^{\text {reg }}=\mathfrak{g}^{\text {reg }} \cap u$. On fixe aussi des mesures de Haar sur $\mathfrak{n}$ et $K$, respectivement $d n$ et $d k$, de masse 1 pour cette dernière. Le choix de la mesure $d n$ sera précisé ultérieurement. 
DÉfinition 1.1.2. - Une partie $P$ de $\mathfrak{g}$ sera dite compacte modulo $G$ si elle est fermée $G$-invariante et si $P \cap \mathfrak{h}$ est compacte pour tout $\mathfrak{h} \in \operatorname{Car}(\mathfrak{g})$.

DÉfinition 1.1.3. - On note $I_{c}(\mathfrak{g})$ l'ensemble des fonctions $\phi$ lisses sur $\mathfrak{g}^{\text {reg }}$ dont le support dans $\mathfrak{g}$ est une partie compacte modulo $G$ telles que

$$
\sup _{x \in \mathfrak{h}^{\text {reg }}}|\partial(w) \phi(x)|<\infty
$$

pour tout $\mathfrak{h} \in \operatorname{Car}(\mathfrak{g})$ et $w \in \operatorname{Sym}\left(\mathfrak{h}_{\mathbb{C}}\right)$. Pour une partie $P$ compacte modulo $G$, on note $I_{P}$ le sous-espace des fonctions de $I_{c}$ dont le support est inclus dans $P$. On considère sur ce sous-espace la topologie définie par les semi-normes

$$
p_{\mathfrak{h}, w}(\phi)=\sup _{x \in \mathfrak{h}^{\text {reg }}}|\partial(w) \phi(x)|
$$

pour $w \in \operatorname{Sym}\left(\mathfrak{h}_{\mathbb{C}}\right)$ et $\mathfrak{h} \in \operatorname{Car}(\mathfrak{g})$. Sur $I_{c}(\mathfrak{g})$, on considère la topologie de limite inductive $\lim _{P} I_{P}(\mathfrak{g})$.

Pour $\mathfrak{h} \in \operatorname{Car}(\mathfrak{g})$ et $x \in \mathfrak{h}^{\text {reg }}$, on note

$$
D_{\mathfrak{g}}(x)=\left|\operatorname{det}\left(\left.\operatorname{ad}(x)\right|_{\mathfrak{g} / \mathfrak{h}}\right)\right|^{1 / 2} .
$$

Sur $\mathfrak{g}^{\text {'reg, }}$, la fonction similaire est notée $D_{\mathfrak{g}^{\prime}}$.

DÉfinition 1.1.4. - Pour $\phi \in \mathcal{D}(\mathfrak{g})$, on considère la fonction $\mathfrak{I}(\phi)$ définie sur $\mathfrak{g}^{\text {reg }}$ par

$$
\mathfrak{I}(\phi)(x)=D_{\mathfrak{g}}(x) \int_{G / H} \phi(g \cdot x) d \dot{g}
$$

pour $x \in \mathfrak{h}^{\text {reg }}$ avec $\mathfrak{h} \in \operatorname{Car}(\mathfrak{g})$.

On a la proposition suivante dû à Harish-Chandra :

Proposition 1.1.5 ([2, Lemme 3.2.2]). - Pour $\phi \in \mathcal{D}(\mathfrak{g})$, la fonction $\mathfrak{I}(\phi)$ appartient à $I_{c}(\mathfrak{g})$, de plus l'application

$$
\begin{aligned}
\mathfrak{I}: \mathcal{D}(\mathfrak{g}) & \longrightarrow I_{c}(\mathfrak{g}) \\
\phi & \longmapsto \mathfrak{I}(\phi)
\end{aligned}
$$

est continue. On utilisera aussi dans la suite $I_{c}\left(\mathfrak{g}^{\prime}\right)$ et $\mathfrak{I}(\phi)$ pour $\phi \in \mathcal{D}\left(\mathfrak{g}^{\prime}\right)$. On souhaite à présent rappeler la définition de Chc. Pour une distribution $u$, on note $W F(u)$ le front d'onde de $u$. Pour $x \in \mathbb{R}$, on pose

$$
\chi(x)=e^{2 \mathrm{i} \pi x} .
$$

Pour un sous-espace vectoriel $u$ de $\mathfrak{s p}$, on considère l'application moment $\tau_{u}: W \rightarrow u^{*}$ définie par

$$
\tau_{u}(w)(x)=\langle x(w), w\rangle \quad x \in u, w \in W .
$$

Et enfin, on pose pour $w \in W$

$$
\chi_{x}(w)=\chi\left(\frac{1}{4} \tau_{\mathfrak{s p}}(w)(x)\right) .
$$


Proposition 1.1.6 ([6, Lemme 1.7]). - Soit $\mathfrak{a}^{\prime \prime}$ l'algèbre de Lie de $A^{\prime \prime}$. Pour tout $\psi \in \mathcal{D}\left(\mathfrak{a}^{\prime \prime}\right)$,

$$
\int_{A^{\prime \prime \prime} \backslash W_{A^{\prime \prime \prime}}}\left|\int_{\mathfrak{a}^{\prime \prime}} \psi(x) \chi_{x}(w) d x\right| d \dot{w}<\infty
$$

On pose

$$
C h c(\psi)=\int_{A^{\prime \prime \prime} \backslash W_{A^{\prime \prime \prime}}} \int_{\mathfrak{a}^{\prime \prime}} \psi(x) \chi_{x}(w) d x d \dot{w},
$$

Chc est une distribution sur $\mathfrak{a}^{\prime \prime}$ et

$$
W F(C h c)=\left\{\left(x, \tau_{\mathfrak{a}^{\prime \prime}}(w)\right) \mid x(w)=0, \quad x \in \mathfrak{a}^{\prime \prime} \text { et } w \in W\right\} .
$$

Proposition 1.1.7 ([6, Proposition 1.8]). - Pour $\mathfrak{h}^{\prime} \in \operatorname{Car}\left(\mathfrak{g}^{\prime}\right)$ et $x^{\prime} \in \mathfrak{h}^{\text {,reg }}$, l'intersection de $W F(C h c)$ avec le fibré conormal de l'injection

$$
\begin{aligned}
i_{x^{\prime}}: \mathfrak{g} & \longrightarrow \mathfrak{a}^{\prime \prime} \\
x & \longmapsto x^{\prime}+x
\end{aligned}
$$

est vide.

Remarque. - Ce résultat montre que l'on peut définir l'image réciproque de Chc par l'injection $i_{x^{\prime}}$ d'après [3, Théorème 8.2.4].

DÉfinition 1.1.8. - On note $C h c_{x^{\prime}}$ l'image réciproque de $C h c$ par $i_{x^{\prime}}$ et pour $\phi \in$ $\mathcal{D}(\mathfrak{g}), \mathbf{C h c}(\phi)$ représente la fonction définie sur $\mathfrak{g}^{\text {reg }}$ par

$$
\operatorname{Chc}(\phi)\left(x^{\prime}\right)=C h c_{x^{\prime}}(\phi) .
$$

Remarque. - La définition de Chc est identique pour les paires de type I.

\subsection{Résultats pour les paires de type II}

On considère l'application

$$
\begin{aligned}
\operatorname{Res}: \mathcal{D}(\mathfrak{g}) & \longrightarrow \mathcal{D}(\mathfrak{l}) \\
\psi & \longmapsto \psi_{\mathfrak{n}}^{K}
\end{aligned}
$$

où

$$
\psi_{\mathfrak{n}}^{K}(x)=\int_{\mathfrak{n}, K} \psi(k \cdot(x+n)) d n d k \text { pour } x \in \mathfrak{l} .
$$

Il est bien connu que cette application est continue (cf. [11, p.53]). Le choix de la mesure $d n$ est tel que

$$
\int_{G / H} \psi(g \cdot x) d \dot{g}=\frac{1}{\left|\operatorname{det}\left(\left.a d(x)\right|_{\mathrm{n}}\right)\right|} \int_{L / H} \psi_{\mathrm{n}}^{K}(l \cdot x) d i
$$

pour tout sous-groupe de Cartan $H$ de $L$ avec $x \in \mathfrak{h}^{\text {reg }}$ où $\mathfrak{h}$ est l'algèbre de Lie de $H$. 
Proposition 1.2.1 ([6, Proposition 7.21]). - Pour $\psi \in \mathcal{D}(\mathfrak{g}), \mathfrak{h}^{\prime} \in \operatorname{Car}\left(\mathfrak{g}^{\prime}\right)$ et $x^{\prime} \in$ $\mathfrak{h}^{\text {reg }}$, on a

$$
\operatorname{Chc}(\psi)\left(x^{\prime}\right)=\int_{G^{\prime} / H^{\prime}} \int_{\operatorname{End}(U)} \operatorname{Res}(\psi)\left(g^{\prime} \cdot x^{\prime}+x\right) d x d \dot{g}^{\prime} .
$$

ThÉORÈme 1.2.2. - Pour $\psi \in \mathcal{D}(\mathfrak{g})$, il existe une fonction $\theta \in \mathcal{D}\left(\mathfrak{g}^{\prime}\right)$ telle que

$$
D_{\mathfrak{g}^{\prime}}\left(x^{\prime}\right) \mathbf{C h c}(\psi)\left(x^{\prime}\right)=\mathfrak{I}(\theta)\left(x^{\prime}\right)
$$

pour tout $x^{\prime} \in \mathfrak{g}^{\prime \text { reg }}$. De plus, l'application

$$
\begin{aligned}
\mathcal{D}(\mathfrak{g}) & \longrightarrow I_{c}\left(\mathfrak{g}^{\prime}\right) \\
\phi & \longmapsto\left(x^{\prime} \in \mathfrak{g}^{\prime \mathrm{reg}} \mapsto D_{\mathfrak{g}^{\prime}}\left(x^{\prime}\right) \mathbf{C h c}(\phi)\left(x^{\prime}\right)\right)
\end{aligned}
$$

est continue.

Démonstration. - Posons

$$
\theta\left(x^{\prime}\right)=\int_{\operatorname{End}(U)} \operatorname{Res}(\psi)\left(x^{\prime}+x\right) d x
$$

pour $x^{\prime} \in \mathfrak{g}^{\prime}$. On a alors $\theta \in \mathcal{D}\left(\mathfrak{g}^{\prime}\right)$ et l'application

$$
\begin{aligned}
\mathcal{D}(\mathfrak{g}) & \longrightarrow \mathcal{D}\left(\mathfrak{g}^{\prime}\right) \\
\psi & \longmapsto \theta
\end{aligned}
$$

est continue. D'après la proposition 1.2 .1 , on a la relation

$$
D_{\mathfrak{g}^{\prime}}\left(x^{\prime}\right) \mathbf{C h c}(\psi)\left(x^{\prime}\right)=\mathfrak{I}(\theta)
$$

pour $x^{\prime} \in \mathfrak{g}^{\prime \text { reg }}$. D'après la proposition 1.1.5, on obtient la continuité de l'application

$$
\psi \longmapsto \Im(\theta)
$$

d'où le résultat. 


\section{CHAPITRE 2}

\section{DÉFINITION DE Chc POUR LES PAIRES DUALES UNITAIRES}

\subsection{Définitions, notations et rappels}

Dans cette section, après avoir introduit des notations générales en particulier sur les sous-algèbres de Cartan et les systèmes de racines, on rappelle la définition de l'intégrale invariante de Harish-Chandra.

2.1.1. Notations. - Soit $V\left(\operatorname{resp} . V^{\prime}\right)$ un $\mathbb{C}$-espace vectoriel de dimension $\mathrm{n}$ (resp. $\mathrm{n}^{\prime}$ ). On munit $V$ (resp. $V^{\prime}$ ) d'une structure hermitienne $($,$\left.) ) (resp. (,)^{\prime}\right)$ de signature $(p, q)$ (resp. $(r, s))$. On supposera que $p \leqslant q$ et $r \leqslant s$. On pose alors $W=\operatorname{Hom}_{\mathbb{C}}\left(V^{\prime}, V\right)$. On note $G$ (resp. $\left.G^{\prime}\right)$ le groupe des isométries sur l'espace hermitien $(V,()$,$) (resp. \left(V^{\prime},(,)^{\prime}\right)$ ). On désigne par $\mathfrak{g}$ et $\mathfrak{g}^{\prime}$ les algèbres de Lie de $G$ et $G^{\prime}$. Les actions de $G, \mathfrak{g}, G^{\prime}$ et $\mathfrak{g}^{\prime}$ sur $W$ sont définies ainsi :

$$
\begin{array}{ll}
g \cdot w=g \circ w & \text { pour } w \in W \text { et } g \in G \text { ou } \mathfrak{g}, \\
g^{\prime} \cdot w=w \circ g^{\prime-1} & \text { pour } w \in W \text { et } g^{\prime} \in G^{\prime}, \\
g^{\prime} \cdot w=-w \circ g^{\prime} & \text { pour } w \in W \text { et } g^{\prime} \in \mathfrak{g}^{\prime} .
\end{array}
$$

Pour $w \in W$, il existe $w^{*} \in \operatorname{Hom}_{\mathbb{C}}\left(V, V^{\prime}\right)$ unique tel que :

$$
\left(w\left(v^{\prime}\right), v\right)=\mathrm{i}\left(v^{\prime}, w^{*}(v)\right)^{\prime} \quad \forall v \in V \text { et } v^{\prime} \in V^{\prime} .
$$

On pose

$$
\left\langle w, w^{\prime}\right\rangle=\operatorname{Tr}_{\mathbb{R}}\left(w^{\prime *} w\right) \quad w, w^{\prime} \in W .
$$

$W$ muni de $\langle$,$\rangle est alors un \mathbb{R}$-espace symplectique de dimension $2 \mathbf{n} \cdot \mathbf{n}^{\prime}$. On note $S p=$ $S p(W)$ le groupe symplectique associé, son algèbre de Lie s'identifie à $\mathfrak{s p}=\mathfrak{s p}(W)$. On fixe une forme bilinéaire $S p$-invariante $\kappa$ non dégénérée sur $\mathfrak{s p}$. Sur chaque sousgroupe $L$ de $S p$ d'algèbre de Lie $\mathfrak{l}$ où la restriction de $\kappa$ à $\mathfrak{l}$ est non dégénérée, on note $\kappa_{\mathfrak{l}}$ cette restriction et on considère sur $L$ et $\mathfrak{l}$ les mesures associées à $\kappa_{\mathfrak{l}}$. Les actions définies précédemment montrent que $G, \mathfrak{g}, G^{\prime}$ et $\mathfrak{g}^{\prime}$ peuvent être considérés comme des sous-groupes de $S p(W)$ ou des sous-algèbres de $\mathfrak{s p}(W)$. On montre alors que $\left(G, G^{\prime}\right)$ 
est une paire duale irréductible dans $S p(W)$ au sens de Howe, [4] et [5]. La forme $\kappa$ induit sur $\mathfrak{g}$ et $\mathfrak{g}^{\prime}$ des formes invariantes et non dégénérées. Pour un sous-groupe de Cartan $H^{\prime}$ de $G^{\prime}$, on note $T^{\prime}$ (resp. $A^{\prime}$ ) sa partie compacte (resp. sa partie déployée) c'est à dire les éléments de $H^{\prime}$ qui ont leurs valeurs propres de module 1 (resp. réelles positives ). On rappelle que $A^{\prime \prime}=S p^{A^{\prime}}$ désigne le centralisateur de $A^{\prime}$ dans $S p$; de même, on note $A^{\prime \prime \prime}$ le centralisateur de $A^{\prime \prime}$ dans $S p$. On montre alors qu'il existe un ouvert dense de $W$ que l'on note $W_{A^{\prime \prime \prime}}$ tel que $A^{\prime \prime \prime} \backslash W_{A^{\prime \prime \prime}}$ soit une variété (cf. [6, p. 302]). D'après ce qui précède, $A^{\prime \prime \prime}$ possède une mesure déterminée que l'on note $d a$; la mesure quotient $d a \backslash d w$ sur $A^{\prime \prime \prime} \backslash W_{A^{\prime \prime \prime}}$ sera notée $d \dot{w}$. Pour tout sous-espace $u$ de $\mathfrak{g}$ (resp. de $\mathfrak{g}^{\prime}$ ), on note $u^{\text {reg }}$ l'ensemble des éléments semi-simples réguliers de $u$ dans $\mathfrak{g}$ $\left(\right.$ resp. $\left.\mathfrak{g}^{\prime}\right)$. L'ensemble $\operatorname{Car}(\mathfrak{g})$ (resp. $\operatorname{Car}\left(\mathfrak{g}^{\prime}\right)$ ) désigne l'ensemble des sous-algèbres de Cartan de $\mathfrak{g}$ (resp. $\left.\mathfrak{g}^{\prime}\right)$.

Soient $X$ un $\mathbb{R}$-espace vectoriel de dimension finie, $U$ un ouvert de $X$ et $p \in \mathbb{N} \cup\{\infty\}$. On désigne par $X_{\mathbb{C}}$ le complexifié de $X$ et $\mathcal{E}(X, p)$ les éléments de $\operatorname{Sym}\left(X_{\mathbb{C}}\right)$ de degré inférieur à $p$. On identifie l'algèbre des opérateurs différentiels à coefficients constants de $X$ avec $\operatorname{Sym}\left(X_{\mathbb{C}}\right)$. On note $\mathcal{C}^{p}(U)$ l'espace des fonctions de classe $C^{p}$ sur $U$. Pour une partie compacte $\mathcal{K}$ de $U$, on note $\mathcal{C}_{c}^{p}(U)$ (resp. $\left.\mathcal{C}_{\mathcal{K}}^{p}(U)\right)$ le sous-espace de $\mathcal{C}^{p}(U)$ des fonctions à support borné (resp. dans $\mathcal{K}$ ). Pour $\phi \in \mathcal{C}_{c}(U)$, on note $\operatorname{Supp}(\phi)$ le support de $\phi$. Sur $\mathcal{C}_{\mathcal{K}}^{p}(U)$, on considère la topologie définie par les semi-normes

$$
p_{w}(\phi)=\sup _{x \in U}|\partial(w) \phi(x)|
$$

pour $w \in \mathcal{E}(X, p)$. On considère $\operatorname{sur} \mathcal{C}_{c}^{p}(U)$ la topologie de limite inductive $\lim _{\mathcal{K}} \mathcal{C}_{\mathcal{K}}^{p}(U)$. Si $p=\infty$, on notera les espaces $\mathcal{C}_{c}^{\infty}(U)$ et $\mathcal{C}_{\mathcal{K}}^{\infty}(U)$ respectivement $\mathcal{D}(U)$ et $\mathcal{D}_{\mathcal{K}}(U)$.

Soient $\mathcal{K}$ une partie compacte de $X$ et $p \in \mathbb{N} \cup\{\infty\}$. On note $\mathcal{H}(U, p)$ (resp. $\mathcal{H}_{c}(U, p)$, $\left.\mathcal{H}_{\mathcal{K}}(U, p)\right)$ le sous-espace de $\mathcal{C}^{p}(U)$ (resp. $\left.\mathcal{C}_{c}^{p}(U), \mathcal{C}_{\mathcal{K}}^{p}(U)\right)$ des fonctions $\phi$ telles que $\partial(w) \phi$ se prolonge par continuité sur l'adhérence de chaque composante connexe de $U$ dans $E$ pour tout $w \in \mathcal{E}(X, p)$. On considère sur $\mathcal{H}_{\mathcal{K}}(U, p)$ la topologie définie par les semi-normes

$$
p_{w}(\phi)=\sup _{x \in U}|\partial(w) \phi(x)|
$$

avec $w \in \mathcal{E}(X, p)$. On considère sur $\mathcal{H}_{c}(U, p)$ la topologie de limite inductive $\lim _{\mathcal{K}} \mathcal{H}_{\mathcal{K}}(U, p)$. Sur l'espace $\mathcal{H}(U, p)$, on considère la topologie définie par les seminormes

$$
p_{w, \mathcal{K}}(\phi)=\sup _{x \in \mathcal{K}}|\partial(w) \phi(x)|
$$

2.1.2. Systèmes de racines et système de représentants de $\operatorname{Car}(\mathfrak{g})$. - Pour $\mathfrak{h} \in \operatorname{Car}(\mathfrak{g})$, on note $\Sigma(\mathfrak{h})$ (resp. $\Sigma^{i}(\mathfrak{h}), \Sigma^{\text {nc }}(\mathfrak{h})$ ), l'ensemble des racines (resp. racines imaginaires, racines imaginaires non compactes) de $\mathfrak{h}$; on considère les ouverts suivants $: \mathfrak{h}^{\text {reg }}$ (l'ensemble des éléments semi-simples réguliers de $\mathfrak{h}$ ) et $\mathfrak{h}^{\text {nc }}$ l'ensemble

$$
\left\{x \in \mathfrak{h} \mid \alpha(x) \neq 0 \forall \alpha \in \Sigma^{\mathrm{nc}}(\mathfrak{h})\right\} .
$$


Il existe une base orthogonale $\left(f_{i}\right)_{1 \leqslant i \leqslant n}\left(\right.$ resp. $\left.\left(f_{i}^{\prime}\right)_{1 \leqslant i \leqslant n}\right)$ de $V$ (resp. $\left.V^{\prime}\right)$ telle que

$$
\left(f_{i}, f_{i}\right)= \begin{cases}1 & \text { si } 1 \leqslant i \leqslant \mathrm{p} \\ -1 & \text { si } \mathrm{p}<i \leqslant \mathrm{n}\end{cases}
$$

et

$$
\left(f_{i}^{\prime}, f_{i}^{\prime}\right)= \begin{cases}1 & \text { si } 1 \leqslant i \leqslant \mathrm{r} \\ -1 & \text { si } \mathrm{r}<i \leqslant \mathrm{n}^{\prime}\end{cases}
$$

L'espace $V$ (resp. $\left.V^{\prime}\right)$ s'identifie alors avec $\mathbb{C}^{\text {n }}\left(\right.$ resp. $\left.\mathbb{C}^{\mathrm{n}^{\prime}}\right)$ et $\mathfrak{g}$ avec :

$$
\left\{z \in \mathfrak{g l}_{\mathrm{n}}(\mathbb{C}) \mid z I_{\mathrm{p}, \mathrm{q}}+I_{\mathrm{p}, \mathrm{q}}{ }^{t} \bar{z}=0\right\} \quad \text { où } I_{\mathrm{p}, \mathrm{q}}=\left(\begin{array}{cc}
I_{\mathrm{p}} & 0 \\
0 & -I_{\mathrm{q}}
\end{array}\right) .
$$

De même, $\mathfrak{g}^{\prime}$ s'identifie avec

$$
\left\{z \in \mathfrak{g l}_{\mathrm{n}^{\prime}}(\mathbb{C}) \mid z I_{\mathrm{r}, \mathrm{s}}+I_{\mathrm{r}, \mathrm{s}}{ }^{t} \bar{z}=0\right\} \quad \text { où } I_{\mathrm{r}, \mathrm{s}}=\left(\begin{array}{cc}
I_{\mathrm{r}} & 0 \\
0 & -I_{\mathrm{s}}
\end{array}\right) .
$$

On note $\mathfrak{h}_{\varnothing}$ la sous-algèbre de Cartan diagonale (et compacte) de $\mathfrak{g}$ et $E_{i, j}$ l'élément de $\mathfrak{g l}_{\mathrm{n}}(\mathbb{C})$ qui vaut 1 en $(i, j)$ et 0 ailleurs. On désigne par $\left(e_{i}\right)_{1 \leqslant i \leqslant n}$ la base duale de $\left(E_{i, i}\right)_{1 \leqslant i \leqslant n}$ dans $\mathfrak{h}_{\varnothing, \mathbb{C}}\left(\right.$ la complexifiée de $\left.\mathfrak{h}_{\varnothing}\right)$. Alors

$$
\Phi\left(\mathfrak{h}_{\varnothing}\right)=\left\{e_{i}-e_{j} \mid 1 \leqslant i<j \leqslant \mathrm{n}\right\}
$$

constitue un système de racines positives pour $\mathfrak{h}_{\varnothing, \mathbb{C}}$. L'ensemble des racines (imaginaires) non compactes de $\Phi\left(\mathfrak{h}_{\varnothing}\right)$ est égal à

$$
\Phi^{\mathrm{nc}}\left(\mathfrak{h}_{\varnothing}\right)=\left\{e_{i}-e_{j} \mid 1 \leqslant i \leqslant p<j \leqslant \mathrm{n}\right\}=\Sigma^{\mathrm{nc}}\left(\mathfrak{h}_{\varnothing}\right) \cap \Phi\left(\mathfrak{h}_{\varnothing}\right) .
$$

On considère la fonction polynomiale sur $\mathfrak{h}_{\varnothing, \mathbb{C}}$ :

$$
\pi_{\mathfrak{h} \varnothing}=\prod_{1 \leqslant i<j \leqslant n}\left(e_{i}-e_{j}\right) \quad \text { (le produit des racines positives). }
$$

Pour $1 \leqslant j \leqslant \mathrm{p}$, on note $\alpha_{j}=e_{j}-e_{j+\mathrm{p}} \in \Phi^{\mathrm{nc}}\left(\mathfrak{h}_{\varnothing}\right), \mathcal{S}_{m}=\left\{\alpha_{1}, \ldots, \alpha_{m}\right\}$ pour $1 \leqslant m \leqslant$ p et $\mathcal{S}_{0}=\varnothing$. Le choix des $\alpha_{j}$ est tel que $\mathcal{S}_{m}$ est un système de racines fortement orthogonales. Pour $1 \leqslant j \leqslant \mathrm{p}$, on note $P_{j}=E_{j, j+\mathrm{p}}+E_{j+\mathrm{p}, j}$ et $A_{j}=\mathrm{i}\left(E_{j, j}+E_{\mathrm{p}+j, \mathrm{p}+j}\right) ;$ on pose alors

$$
\mathfrak{h}_{\mathcal{S}_{m}}=\oplus_{j=1}^{m} \mathbb{R} P_{j} \oplus_{j=1}^{m} \mathbb{R} A_{j} \oplus \underset{\substack{m<j \leqslant \mathrm{p} \\ \text { ou } \mathrm{p}+m<j \leqslant \mathrm{n}}}{m i E_{j, j}} .
$$

La famille $\left(\mathfrak{h}_{\mathcal{S}_{m}}\right)_{0 \leqslant m \leqslant p}$ constitue un système de représentants de sous-algèbres de Cartan de $\mathfrak{g}$. On considère sur $\mathfrak{h}_{\mathcal{S}_{m}}$ le système de coordonnées associées suivant :

$$
\left(u_{j}\right)_{1 \leqslant j \leqslant m}, \quad\left(v_{j}\right)_{1 \leqslant j \leqslant m} \quad \text { et } \quad\left(x_{j}\right)_{\substack{m<j \leqslant \mathrm{p} \\ \text { ou } \mathrm{p}+m<j \leqslant \mathrm{n}}} .
$$


Ensuite, on définit l'application $c_{\mathcal{S}_{m}}$ appelée transformée de Cayley de $\mathfrak{h}_{\varnothing, \mathbb{C}}$ dans $\mathfrak{h}_{\mathcal{S}_{m}, \mathbb{C}}$ ainsi :

$$
\left(c_{\mathcal{S}_{m}}^{-1}(x)\right)_{j}= \begin{cases}-\mathrm{i} u_{j}+v_{j} & \text { si } 1 \leqslant j \leqslant m \\ \mathrm{i} u_{j-\mathrm{p}}+v_{j-\mathrm{p}} & \text { si } \mathrm{p}<j \leqslant \mathrm{p}+m \\ x_{j} & \text { si } m<j \leqslant \mathrm{p} \text { ou } \mathrm{p}+m<j \leqslant \mathrm{n} .\end{cases}
$$

On peut vérifier que $c_{\mathcal{S}_{m}}\left(\mathfrak{h}_{\varnothing, \mathbb{C}}\right) \cap \mathfrak{g}=\mathfrak{h}_{\mathcal{S}_{m}}$. On considère aussi pour $\mathfrak{g}^{\prime}$, la sous-algèbre de Cartan diagonale $\mathfrak{h}_{\varnothing}^{\prime}$; sur $\mathfrak{h}_{\varnothing, \mathbb{C}}$ et $\mathfrak{h}_{\varnothing, \mathbb{C}}^{\prime}$ les coordonnées sont fournies par les bases $\left(\mathrm{i} E_{j}\right)_{1 \leqslant j \leqslant n}$ et $\left(\mathrm{i} E_{j}\right)_{1 \leqslant j \leqslant \mathrm{n}^{\prime}}$.

2.1.3. Intégrale invariante de Harish-Chandra. - Pour $\mathcal{S}=\mathcal{S}_{0}, \ldots, \mathcal{S}_{p}$, on note $H_{\mathcal{S}}$ le sous-groupe de Cartan de $G$ associé à $\mathfrak{h}_{\mathcal{S}}$ et $\Phi\left(\mathfrak{h}_{\mathcal{S}}\right)$ le système de racines positives issu de $\Phi\left(\mathfrak{h}_{\varnothing}\right)$ par $c_{\mathcal{S}}$. Pour $x \in \mathfrak{h}_{\mathcal{S}}^{\text {reg }}$, on pose

$$
\mathbf{e}_{\mathcal{S}}(x)=\operatorname{sign}\left(\prod_{\alpha \in S} \bar{\alpha} \circ c_{\mathcal{S}}^{-1}(x)\right) .
$$

On a la définition suivante :

DÉFInition 2.1.1. - L'intégrale invariante de Harish-Chandra d'une fonction $\psi \in$ $\mathcal{D}(\mathfrak{g})$ est définie pour $x \in \mathfrak{h}_{\mathcal{S}}^{\text {reg }}$ par

$$
\psi_{\mathcal{S}}(x)=\overline{\pi_{\mathfrak{h} \varnothing}} \circ c_{\mathcal{S}}^{-1}(x) \mathbf{e}_{\mathcal{S}}(x) \int_{G / H_{\mathcal{S}}} \psi\left(g x g^{-1}\right) d \dot{g} .
$$

Les propriétés de ces fonctions sont précisées si $G$ est de rang 2 dans la sous-section 3.1.1 et pour $G$ quelconque dans la sous-section 4.1.2.

\subsection{Formules de calcul de Chc}

La définition de l'intégrale de Cauchy Harish-Chandra pour la paires duale $(\mathfrak{u}(\mathrm{p}, \mathrm{q}), \mathfrak{u}(\mathrm{r}, \mathrm{s}))$ est identique à celle donnée pour les paires de type II dans la première partie.

Dans cette section, on donne une expression de l'intégrale de Cauchy HarishChandra tout d'abord pour les sous-algèbres de Cartan compactes de $\mathfrak{g}^{\prime}$, Théorème 2.2.4 puis pour les sous-algèbres de Cartan quelconque de $\mathfrak{g}^{\prime}$, Théorème 2.2.7. L'expression de Chc dans le premier cas, nécessite l'introduction de fonctions polynomiales qui sont définies dans un premier temps ci-dessous.

Dans toute cette section, on considère $n$ et $n^{\prime}$ deux entiers tels que $n^{\prime} \leqslant n$. 
2.2.1. Préliminaires. - Pour $x \in \mathbb{C}^{\mathrm{n}}$ (resp. $\left.x^{\prime} \in \mathbb{C}^{\mathrm{n}^{\prime}}\right)$, on pose :

$$
\begin{aligned}
& \pi(x)=\left\{\begin{array}{ll}
\prod_{1 \leqslant i<j \leqslant n}\left(x_{i}-x_{j}\right) & \text { si } \mathrm{n}>1 \\
1 & \text { si } \mathrm{n}=1
\end{array},\right. \\
& \pi^{\prime}\left(x^{\prime}\right)=\left\{\begin{array}{ll}
\prod_{1 \leqslant i<j \leqslant \mathrm{n}^{\prime}}\left(x_{i}^{\prime}-x_{j}^{\prime}\right) & \text { si } \mathrm{n}^{\prime}>1 \\
1 & \text { si } \mathrm{n}^{\prime}=1
\end{array},\right. \\
& M_{p}\left(x^{\prime}\right)=\prod_{j=1}^{\mathrm{n}^{\prime}}\left(1+\mathrm{i} x_{j}^{\prime}\right)^{p} \text { pour } p \in \mathbb{N} \text { et } Q_{L}\left(x, x^{\prime}\right)=\prod_{\substack{(i, j) \in\{1, \ldots, \mathrm{n}\} \times\left\{1, \ldots, \mathbf{n}^{\prime}\right\} \\
i \neq L(j)}}\left(x_{i}-x_{j}^{\prime}\right)
\end{aligned}
$$

où $L$ est une application de $\left\{1, \ldots, \mathbf{n}^{\prime}\right\}$ dans $\{1, \ldots, \mathbf{n}\}$. Si $\mathbb{K}$ désigne un des corps $\mathbb{R}$ ou $\mathbb{C}, \mathbb{K}[x]$ (resp. $\mathbb{K}(x)$ ) désigne l'algèbre des fonctions polynomiales (resp. des fractions rationnelles) à coefficient dans $\mathbb{K}$ en $\left(x_{i}\right)_{1 \leqslant i \leqslant n}$. Pour tout ensemble fini $E, \mathfrak{S}(E)$ désigne le groupe des permutations de $E$. Dans la suite de cette sous-section, $L$ désignera toujours une application de $\left\{1, \ldots, \mathrm{n}^{\prime}\right\}$ dans $\{1, \ldots, \mathrm{n}\}$. On définit alors

$$
\begin{aligned}
& \varepsilon(L)=(-1)^{\operatorname{Card}\left\{(i, j) \in\left\{1, \ldots, \mathrm{n}^{\prime}\right\} \times\left\{1, \ldots, \mathrm{n}^{\prime}\right\} \mid i<j, L(i)>L(j)\right\}} \\
& \eta(L)=(-1)^{\operatorname{Card}\{(i, j) \in\{1, \ldots, \mathrm{n}\} \times\{1, \ldots, \mathrm{n}\} \mid j \in \operatorname{im}(L), j<i\}}
\end{aligned}
$$

où $\operatorname{im}(L)$ représente l'image de $L$.

Remarque. - Si $\mathrm{n}=\mathrm{n}^{\prime}$ et $L$ est une application bijective de $\{1, \ldots, \mathrm{n}\}$ dans $\{1, \ldots, \mathrm{n}\}$ alors $\varepsilon(L)$ est la signature de $L$ au sens habituel.

Proposition 2.2.1. - Pour $p \in\left\{0, \ldots, \mathrm{n}-\mathrm{n}^{\prime}\right\}$, il existe une unique famille de fonctions polynomiales $\left(P_{L, p}\right)_{L}$ de $\mathbb{R}[x]$ indexée sur l'ensemble des injections $L$ de $\left\{1, \ldots, \mathrm{n}^{\prime}\right\}$ dans $\{1, \ldots, \mathrm{n}\}$, telle que:

$$
M_{p}\left(x^{\prime}\right) \pi(x) \pi^{\prime}\left(x^{\prime}\right)=\sum_{L} P_{L, p}(x) Q_{L}\left(x, x^{\prime}\right) \quad \forall x \in \mathbb{C}^{\mathrm{n}}, x^{\prime} \in \mathbb{C}^{\mathrm{n}^{\prime}} .
$$

On a de plus les propriétés suivantes :

(1) $P_{L, p}(x)=\varepsilon(L) \eta(L) M_{p}\left(x_{L}^{\prime}\right) \prod_{\substack{1 \leqslant i<j \leqslant n \\ i, j \notin \operatorname{im}(L)}}\left(x_{i}-x_{j}\right)$

où $x_{L}^{\prime}$ est l'élément de $\mathbb{C}^{\mathrm{n}^{\prime}}$ définit par $\left(x_{L}^{\prime}\right)_{i}=x_{L(i)}$ pour $i \in\left\{1, \ldots, \mathrm{n}^{\prime}\right\}$.

(2) $P_{\sigma \cdot L, p}(\sigma \cdot x)=\varepsilon(\sigma) P_{L, p}(x) \forall \sigma \in \mathfrak{S}(\{1, \ldots, \mathrm{n}\})$.

Démonstration. - Première étape : Montrons tout d'abord que la famille $\left(Q_{L}\right)_{L}$ indexée sur l'ensemble des applications de $\left\{1, \ldots, \mathrm{n}^{\prime}\right\}$ dans $\{1, \ldots, \mathrm{n}\}$ est une base $\mathrm{du}$ sous-espace vectoriel $\mathcal{E}\left(\mathrm{n}, \mathrm{n}^{\prime}\right)$ de $\mathbb{C}(x) \otimes \mathbb{C}\left[x^{\prime}\right]$ des fonctions polynomiales $P$ à coefficients dans $\mathbb{C}(x)$ telles que $\operatorname{deg}_{x_{i}^{\prime}}(P) \leqslant \mathrm{n}-1$. Pour $\mathrm{n}^{\prime}=1$, on note $Q_{i}$ pour $Q_{L}$ si $i=L(1)$. En évaluant $Q_{i}(x,$.$) en x_{j}$, on a

$$
Q_{i}\left(x, x_{j}\right)= \begin{cases}\prod_{k \neq j}\left(x_{k}-x_{j}\right) & \text { si } j=i, \\ 0 & \text { sinon. }\end{cases}
$$


On en déduit que $\left(Q_{i}\right)_{i \in\{1, \ldots, \mathrm{n}\}}$ est une base de $\mathcal{E}(\mathrm{n}, 1)$ en tant que $\mathbb{C}(x)$-espace vectoriel. Pour $\mathrm{n}^{\prime}>1$ quelconque, on note pour $i \in\left\{1, \ldots, \mathrm{n}^{\prime}\right\}, \mathcal{E}_{i}(\mathrm{n}, 1)$ l'espace des fonctions polynomiales de $\mathcal{E}(\mathrm{n}, 1)$ en la variable $x_{i}^{\prime}$ (en remplaçant $x_{1}^{\prime}$ par $x_{i}^{\prime}$ ). On obtient le résultat avec ce qui précède en remarquant les points suivants :

(1) $\mathcal{E}\left(\mathrm{n}, \mathrm{n}^{\prime}\right)=\mathcal{E}_{1}(\mathrm{n}, 1) \otimes \cdots \otimes \mathcal{E}_{\mathrm{n}^{\prime}}(\mathrm{n}, 1)$,

(2) $Q_{L}\left(x, x^{\prime}\right)=\prod_{i \in\left\{1, \ldots, \mathrm{n}^{\prime}\right\}} Q_{L(i)}\left(x, x_{i}^{\prime}\right) \forall L:\left\{1, \ldots, \mathrm{n}^{\prime}\right\} \rightarrow\{1, \ldots, \mathrm{n}\}$.

Deuxième étape : Comme $\operatorname{deg}_{x_{i}^{\prime}}\left(M_{p}\left(x^{\prime}\right) \pi^{\prime}\left(x^{\prime}\right)\right) \leqslant \mathrm{n}-1$, on peut considérer les coordonnées $P_{L, p} \in \mathbb{C}(x)$ de $M_{p}\left(x^{\prime}\right) \pi(x) \pi^{\prime}\left(x^{\prime}\right)$ dans cette base, ainsi :

$$
M_{p}\left(x^{\prime}\right) \pi(x) \pi^{\prime}\left(x^{\prime}\right)=\sum_{L:\left\{1, \ldots, \mathrm{n}^{\prime}\right\} \rightarrow\{1, \ldots, \mathrm{n}\}} P_{L, p}(x) Q_{L}\left(x, x^{\prime}\right) .
$$

On considère $j_{1}, j_{2} \in\left\{1, \ldots, \mathbf{n}^{\prime}\right\}$ tels que $j_{1} \neq j_{2}$ et $i_{0} \in\{1, \ldots, \mathbf{n}\}$. En évaluant l'égalité précédente pour les variables $x_{j_{1}}^{\prime}$ et $x_{j_{2}}^{\prime}$ en $x_{i_{0}}$. Le membre de gauche devient nul et on a

$$
\left.Q_{L}\left(x, x^{\prime}\right)\right|_{x_{j_{1}}^{\prime}=x_{i_{0}}} ^{x_{j_{2}}^{\prime}=x_{i_{0}}}= \begin{cases}\prod_{\substack{(i, j) \in\left\{1, \ldots, \mathbf{n}^{\prime}\right\} \times\{1, \ldots, \mathrm{n}\} \\ i \neq L(j), j \neq j_{1} \text { et } j \neq j_{2}}}\left(x_{i}-x_{j}^{\prime}\right)\left(\prod_{i \neq i_{0}}\left(x_{i}-x_{i_{0}}\right)\right)^{2} \\ 0 & \text { si } L\left(j_{1}\right)=L\left(j_{2}\right)=i_{0} \\ 0 & \text { sinon. }\end{cases}
$$

On pose

En notant $\widehat{L}=\left.L\right|_{\left\{1, \ldots, \mathrm{n}^{\prime}\right\} \backslash\left\{j_{1}, j_{2}\right\}}$, on a

$$
\widetilde{Q}_{L}\left(x, x^{\prime}\right)=\left.Q_{L}\left(x, x^{\prime}\right)\right|_{\begin{array}{c}
x_{j_{1}}^{\prime}=x_{i_{0}} \\
x_{j_{2}}^{\prime}=x_{i_{0}}
\end{array}}
$$

$$
\widetilde{Q}_{L}\left(x, x^{\prime}\right)=R Q_{\widehat{L}}\left(x, x^{\prime}\right), \quad \text { où } R(x)=\left(\prod_{i \neq i_{0}}\left(x_{i}-x_{i_{0}}\right)\right)^{2} .
$$

L'évaluation de l'égalité (2.2.1) devient après simplification par $R$ :

$$
\sum_{\substack{L:\left\{1, \ldots, \mathbf{n}^{\prime}\right\} \rightarrow\{1, \ldots, \mathrm{n}\} \\ L\left(j_{1}\right)=L\left(j_{2}\right)=i_{0}}} P_{L, p}(x) Q_{\widehat{L}}\left(x, x^{\prime}\right)=0
$$

L'indépendance de la famille $\left(Q_{\widehat{L}}\right)_{\widehat{L}}$ montre que $P_{L, p}=0$ si $L\left(j_{1}\right)=L\left(j_{2}\right)=i_{0}$. On en déduit que $P_{L, p}=0$ dès que $L$ est non injectif. Pour montrer que $P_{L, p} \in \mathbb{R}[x]$, il suffit d'évaluer l'égalité (2.2.1) en $x^{\prime}$ en posant $x_{j}^{\prime}=x_{L(j)} \forall j \in\left\{1, \ldots, \mathrm{n}^{\prime}\right\}$; on obtient alors :

$$
\begin{aligned}
& P_{L, p}(x)=M_{p}\left(x_{L}^{\prime}\right) \frac{\prod_{i_{1}<i_{2}}\left(x_{i_{1}}-x_{i_{2}}\right) \prod_{j_{1}<j_{2}}\left(x_{L\left(j_{1}\right)}-x_{L\left(j_{2}\right)}\right)}{\prod_{i \neq j, j \in \operatorname{im}(L)}\left(x_{i}-x_{j}\right)} \\
& =M_{p}\left(x_{L}^{\prime}\right) \varepsilon(L) \eta(L) \prod_{\substack{1 \leqslant i<j \leqslant n \\
i, j \notin \operatorname{im}(L)}}\left(x_{i}-x_{j}\right) .
\end{aligned}
$$

On a ainsi le point (1). Le point (2) de la proposition découle des relations suivantes

$$
\pi(\sigma \cdot x)=\varepsilon(\sigma) \pi(x) \quad \text { et } \quad Q_{\sigma \cdot L}\left(\sigma \cdot x, x^{\prime}\right)=Q_{L}\left(x, x^{\prime}\right) .
$$




\section{Corollaire 2.2 .2}

(1) $P_{L, 0}$ ne dépend que des variables $x_{i}$ avec $i \notin \operatorname{im}(L)$.

(2) $S i \mathrm{n}=\mathrm{n}^{\prime}, P_{L, 0}=\varepsilon(L)(-1)^{\mathrm{n}(\mathrm{n}-1) / 2}$.

2.2.2. Calcul de Chc pour une sous-algèbre de Cartan compacte. - On

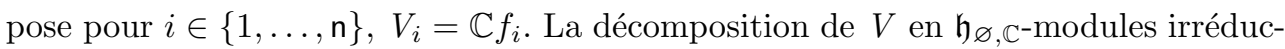
tibles s'écrit

$$
V=\oplus_{1 \leqslant i \leqslant n} V_{i} .
$$

On a le même résultat pour le $\mathfrak{h}_{\varnothing, \mathbb{C}^{-}}^{\prime}$ module $V^{\prime}$ en posant pour $i \in\left\{1, \ldots, \mathrm{n}^{\prime}\right\}, V_{i}^{\prime}=$ $\mathbb{C} f_{i}^{\prime}$. On obtient alors en posant $W_{i, j}=\operatorname{Hom}_{\mathbb{C}}\left(V_{i}^{\prime}, V_{j}\right)$, la décomposition de $W$ en $\mathfrak{h}_{\varnothing, \mathbb{C}} \oplus \mathfrak{h}_{\varnothing, \mathbb{C}}^{\prime}$-modules irréductibles :

$$
W=\oplus_{i, j} W_{i, j} .
$$

Pour chaque espace $A$ muni d'une forme hermitienne de signature $(a, b)$, on pose $\operatorname{sign}(A)=(-1)^{b}$ ainsi, on a

$$
\begin{gathered}
\operatorname{sign}(V)=(-1)^{\mathrm{q}}, \quad \operatorname{sign}\left(V^{\prime}\right)=(-1)^{\mathrm{s}} \\
\operatorname{sign}\left(V_{i}\right)=\left\{\begin{array}{ll}
1 & \text { si } 1 \leqslant i \leqslant \mathrm{p} \\
-1 & \text { si } \mathrm{p}<i \leqslant \mathrm{n}
\end{array} \text { et } \quad \operatorname{sign}\left(V_{i}^{\prime}\right)= \begin{cases}1 & \text { si } 1 \leqslant i \leqslant \mathrm{r} \\
-1 & \text { si } \mathrm{r}<i \leqslant \mathrm{n}^{\prime}\end{cases} \right.
\end{gathered}
$$

On considère les fonctions suivantes pour $x \in \mathfrak{h}_{\varnothing, \mathbb{C}}$ et $x^{\prime} \in \mathfrak{h}_{\varnothing, \mathbb{C}}^{\prime} \operatorname{avec}^{\operatorname{det}_{W}}\left(x+x^{\prime}\right) \neq 0$ :

$$
\begin{aligned}
& \operatorname{chc}^{(i, j)}\left(x+x^{\prime}\right)=\frac{2 \mathrm{i} \operatorname{sign}\left(V_{j}\right) \operatorname{sign}\left(V_{i}^{\prime}\right)}{x_{j}-x_{i}^{\prime}}, \quad \quad \operatorname{chc}^{L}=\frac{1}{\prod_{1 \leqslant i \leqslant \mathrm{n}^{\prime}}\left(x_{L(i)}-x_{i}^{\prime}\right)} \\
& \text { chc }=\prod_{(i, j) \in\{1, \ldots, \mathrm{n}\} \times\left\{1, \ldots, \mathrm{n}^{\prime}\right\}} \operatorname{chc}^{(i, j)} \text { et } \quad M_{p, \mathfrak{h}_{\varnothing}^{\prime}}\left(x^{\prime}\right)=M_{p}\left(x^{\prime}\right) .
\end{aligned}
$$

On note $C_{\mathfrak{g}, \mathfrak{g}^{\prime}}=2^{\mathrm{nn} \mathrm{n}^{\prime}} \operatorname{sign}\left(V^{\prime}\right)^{\mathrm{n}} \operatorname{sign}(V)^{\mathrm{n}^{\prime}} \mathrm{i}^{\mathrm{nn} \mathrm{n}^{\prime}+\frac{\mathrm{n}(\mathrm{n}-1)}{2}-\frac{\mathrm{n}^{\prime}\left(\mathrm{n}^{\prime}-1\right)}{2}}$.

Proposition 2.2.3. - On a pour $x^{\prime} \in \mathfrak{h}_{\varnothing, \mathbb{C}}^{\prime}$ et $x \in \mathfrak{h}_{\varnothing, \mathbb{C}}$ avec $\operatorname{det}_{W}\left(x+x^{\prime}\right) \neq 0$

$$
M_{p, \mathfrak{h}_{\varnothing}^{\prime}}\left(x^{\prime}\right) \overline{\pi_{\mathfrak{h}_{\varnothing}^{\prime}}}\left(x^{\prime}\right) \pi_{\mathfrak{h} \varnothing}(x) \operatorname{chc}\left(x+x^{\prime}\right)=C_{\mathfrak{g}, \mathfrak{g}^{\prime}} \sum P_{L, p}(x) \operatorname{chc}^{L}\left(x+x^{\prime}\right)
$$

où la somme s'effectue sur les injections $L$ de $\left\{1, \ldots, \mathrm{n}^{\prime}\right\}$ dans $\{1, \ldots, \mathrm{n}\}$.

Démonstration. - Cela découle directement de la proposition 2.2.1.

Pour une injection $L$ de $\left\{1, \ldots, \mathrm{n}^{\prime}\right\}$ dans $\{1, \ldots, \mathrm{n}\}$, on considère $y^{L} \in \mathfrak{h}_{\varnothing}$ et $y_{\mathcal{S}}^{L} \in \mathfrak{h}_{\mathcal{S}}$ définis ainsi :

$$
y_{k}^{L}=\left\{\begin{array}{ll}
\operatorname{sign}\left(V_{k}\right) \operatorname{sign}\left(V_{j}^{\prime}\right) & \text { si } k=L(j) \\
0 & \text { sinon }
\end{array} \text { et } y_{\mathcal{S}, k}^{L}=\left\{\begin{array}{ll}
y_{k}^{L} & \text { si } \alpha\left(E_{k, k}\right)=0 \forall \alpha \in \mathcal{S} \\
0 & \text { sinon }
\end{array} .\right.\right.
$$

La forme $\kappa_{\mathfrak{g}}$ fournit la décomposition de Cartan suivante $\mathfrak{g}=\mathbf{k} \oplus \mathfrak{p}$. On a alors $\mathfrak{h}_{\mathcal{S}}=\left(\mathfrak{h}_{\mathcal{S}} \cap \mathbf{k}\right) \oplus\left(\mathfrak{h}_{\mathcal{S}} \cap \mathfrak{p}\right)$. On fixe une chambre de Weyl de $\mathfrak{p} \cap \mathfrak{h}_{\mathcal{S}}$ où $\mathbf{e}_{\mathcal{S}}>0$ que l'on note $\left(\mathfrak{p} \cap \mathfrak{h}_{\mathcal{S}}\right)^{+}$et on pose $\mathfrak{h}_{\mathcal{S}}^{+}=\mathrm{k} \cap \mathfrak{h}_{\mathcal{S}}+\left(\mathfrak{p} \cap \mathfrak{h}_{\mathcal{S}}\right)^{+}$. De plus, on note $n_{\mathcal{S}}$ le quotient 
du cardinal de la restriction de $W\left(H_{\mathcal{S}}\right)$ à $\mathfrak{p} \cap \mathfrak{h}_{\mathcal{S}}$ par le cardinal de $W\left(H_{\mathcal{S}}\right)$ où $W\left(H_{\mathcal{S}}\right)$ désigne le groupe de Weyl de $H_{\mathcal{S}}$. On considère l'application :

$$
\begin{aligned}
C_{\mathcal{S}}^{L}: \mathfrak{h}_{\mathcal{S}}^{+} \times[0,1] & \longrightarrow \mathfrak{h}_{\mathcal{S}, \mathbb{C}} \quad\left(\text { la complexifiée de } \mathfrak{h}_{\mathcal{S}}\right) \\
(x, t) & \longmapsto x+\mathrm{i} t y_{\mathcal{S}}^{L}
\end{aligned}
$$

On étend la définition de $\Phi^{\mathrm{nc}}\left(\mathfrak{h}_{\varnothing}\right)$ aux sous-algèbres de Cartan $\mathfrak{h}_{\mathcal{S}}$ en posant :

$\Phi^{\text {nc }}\left(\mathfrak{h}_{\mathcal{S}}\right)=\left\{\alpha \in \Phi\left(\mathfrak{h}_{\varnothing}\right) \mid \alpha \circ c_{\mathcal{S}}^{-1}\right.$ est une racine imaginaire non compacte de $\left.\mathfrak{h}_{\mathcal{S}}\right\}$

Pour $N \in \mathbb{N}$ et $v \in \operatorname{Sym}\left(\mathfrak{g}_{\mathbb{C}}\right)$, on pose :

$$
q_{N}(v)=\sum_{p=0}^{N} \frac{v^{p}}{p !} \in \operatorname{Sym}\left(\mathfrak{g}_{\mathbb{C}}\right)
$$

On rappelle que l'on identifie les opérateurs différentiels à coefficients constants de $\mathfrak{g}$ avec $\operatorname{Sym}\left(\mathfrak{g}_{\mathbb{C}}\right)$. Pour une fonction $\psi$ lisse sur $\mathfrak{g}^{\text {reg }}$ et $N \in \mathbb{N}$, l'extension de degré $N$ (cf. [6, Appendice $A]$ ) de $\psi$ est la fonction $\psi_{N}$ définie sur l'ensemble

$$
\left\{u+\mathrm{i} v \mid u \in \mathfrak{h}_{\mathcal{S}}^{\text {reg }}, v \in \mathfrak{h}_{\mathcal{S}}\right\}
$$

que l'on note $\mathfrak{h}_{\mathcal{S}, \mathbb{C}}^{\text {reg }}$ par

$$
\psi_{N}(u+\mathrm{i} v)=q_{N}(\mathrm{i} v) \psi(u) .
$$

On note ainsi $\psi_{\mathcal{S}, N}$ l'extension de degré $N$ de $\psi_{\mathcal{S}}$ définie sur $\mathfrak{h}_{\mathcal{S}, \mathbb{C}}^{\text {reg }}$. Pour $\alpha \in \Phi^{\text {nc }}\left(\mathfrak{h}_{\mathcal{S}}\right)$, $\left.C_{\mathcal{S}}^{L}\right|_{\alpha}$ représente la restriction de $C_{\mathcal{S}}^{L}$ à $\operatorname{ker}\left(\alpha \circ c_{\mathcal{S}}^{-1}\right) \times[0,1]$; de même, $C_{\mathcal{S}}^{L}(1)$ représente la restriction de $C_{\mathcal{S}}^{L}$ pour $t=1$. On considère aussi sur $\mathfrak{h}_{\mathcal{S}, \mathbb{C}}$

$$
\nu_{L, x^{\prime}, \mathcal{S}, N}(z)=P_{L, p}\left(c_{\mathcal{S}}^{-1}(z)\right) \operatorname{chc}^{L}\left(c_{\mathcal{S}}^{-1}(z)+x^{\prime}\right) \cdot \psi_{\mathcal{S}, N}(z) \mu_{\mathcal{S}}
$$

où $\mu_{\mathcal{S}}$ désigne la mesure induite par $\kappa_{\mathfrak{g}}$ sur $\mathfrak{h}_{\mathcal{S}}$ en considérant la base de la décomposition (2.1.1) directe.

THÉorème 2.2.4 ([6, Théorème 10.19]). - Pour $x^{\prime} \in \mathfrak{h}_{\varnothing}^{\text {reg }}$ et $N \geqslant 0$ assez grand, on a

$$
\begin{aligned}
& M_{p, \mathfrak{h}_{\varnothing}^{\prime}}\left(x^{\prime}\right) \overline{\pi_{\mathfrak{h}^{\prime}}}\left(x^{\prime}\right) \mathbf{C h c}(\psi)\left(x^{\prime}\right) \\
& \quad=C_{\mathfrak{g}, \mathfrak{g}^{\prime}} \sum n_{\mathcal{S}}\left(\int_{C_{\mathcal{S}}^{L}(1)} \nu_{L, x^{\prime}, \mathcal{S}, N}-\int_{C_{\mathcal{S}}^{L}} d\left(\nu_{L, s^{\prime}, \mathcal{S}, N}\right)-\sum_{\alpha \in \Phi_{\mathcal{S}}^{\text {nc }}} \int_{\left.C_{\mathcal{S}}^{L}\right|_{\alpha}} \nu_{L, x^{\prime}, \mathcal{S}, N}\right)
\end{aligned}
$$

où la somme s'effectue sur les injections $L$ de $\left\{1, \ldots, \mathrm{n}^{\prime}\right\}$ dans $\{1, \ldots, \mathrm{n}\}$ et la famille de systèmes de racines fortement orthogonales $\mathcal{S}=\mathcal{S}_{0}, \ldots, \mathcal{S}_{\mathrm{p}}$. Chaque intégrale est absolument convergente.

On note $\nu_{L, x^{\prime}, \mathcal{S}}=\nu_{L, x^{\prime}, \mathcal{S}, 0}$. Le théorème précédent permet d'obtenir l'expression suivante : 
Corollaire 2.2.5. - Pour $x^{\prime} \in \mathfrak{h}_{\varnothing}^{\text {reg }}$, on a

$$
M_{p, \mathfrak{h}_{\varnothing}^{\prime}}\left(x^{\prime}\right) \overline{\pi_{\mathfrak{h}_{\varnothing}^{\prime}}}\left(x^{\prime}\right) \mathbf{C h c}(\psi)\left(x^{\prime}\right)=C_{\mathfrak{g}, \mathfrak{g}^{\prime}} \sum n_{\mathcal{S}} \lim _{t \rightarrow 0, t>0} \int_{C_{\mathcal{S}}^{L}(t)} \nu_{L, x^{\prime}, \mathcal{S}}
$$

où la somme s'effectue sur les injections $L$ de $\left\{1, \ldots, \mathrm{n}^{\prime}\right\}$ dans $\{1, \ldots, \mathrm{n}\}$ et la famille de systèmes de racines fortement orthogonales $\mathcal{S}=\mathcal{S}_{0}, \ldots, \mathcal{S}_{\mathrm{p}}$.

Démonstration. - On obtient cette égalité en considérant l'égalité $A .3$ de l'appendice $A$ de $[6]$.

2.2.3. Calcul de Chc pour une sous-algèbre de Cartan quelconque. - On considère $H^{\prime}$ un sous-groupe de Cartan quelconque de $G^{\prime}$. Sa décomposition de Cartan s'écrit $H^{\prime}=T^{\prime} A^{\prime}$. On pose alors $V_{c}^{\prime}=\left\{x \in V^{\prime} \mid a \cdot x=x \forall a \in A^{\prime}\right\}$ et $V_{s}^{\prime}=V_{c}^{\prime \perp}$ son orthogonal dans $V^{\prime}$. Il existe alors deux lagrangiens $X^{\prime}$ et $Y^{\prime}$ de $V_{s}^{\prime}, H^{\prime}$-invariants tels que $V_{s}^{\prime}=X^{\prime} \oplus Y^{\prime}$.

Proposition 2.2.6 ([6, Lemme 11.11]). - On a l'alternative suivante :

(1) Si $V$ ne contient pas un sous-espace isotrope de dimension égale à $\operatorname{dim}_{\mathbb{C}} X^{\prime}$ alors $\mathbf{C h c}(\psi)\left(x^{\prime}\right)=0$ pour $x^{\prime} \in \mathfrak{h}^{\text {reg }}$ et $\psi \in \mathcal{D}(\mathfrak{g})$.

(2) Sinon, on peut supposer que $V_{s}^{\prime} \subset V$ et la restriction de (, ) à $V_{s}^{\prime}$ est non dégénérée.

On suppose jusqu'à la fin de cette sous-section que l'on se trouve dans la deuxième situation. On adopte les notations suivantes. On pose $U=V_{s}^{\prime \perp}(\subset V)$ et $G(U)$ le groupe des isométries associé à $(U,()$,$) ; on note \mathfrak{g}(U)$ son algèbre de Lie. On a les injections naturelles suivantes $\operatorname{End}\left(X^{\prime}\right) \hookrightarrow \mathfrak{g}, \operatorname{Hom}\left(U, X^{\prime}\right) \hookrightarrow \mathfrak{g}$ et $\mathfrak{g}(U) \hookrightarrow \mathfrak{g}$. Enfin, on note $\mathfrak{n}$ (resp. $\mathfrak{n}^{\prime}$ ) le radical unipotent de la sous-algèbre de $\mathfrak{g}$ (resp. $\mathfrak{g}^{\prime}$ ) préservant $X^{\prime}$ et $K$ un sous-groupe compact maximal de $G$. On considère sur $K$ la mesure de Haar de masse 1 . On rappelle qu'il existe une mesure de Haar $d n$ sur $\mathfrak{n}$ telle que :

$$
\int_{G / H} \psi(g \cdot x) d \dot{g}=\frac{1}{\left|\operatorname{det}\left(\left.\operatorname{ad}(x)\right|_{\mathfrak{n}}\right)\right|} \int_{L / H} \psi_{\mathfrak{n}}^{K}(l \cdot x) d \dot{l} \text { pour } x \in \mathfrak{h}^{\text {reg }}
$$

pour tout $\mathfrak{h} \in \operatorname{Car}(\mathfrak{l})$ et $\psi \in \mathcal{D}(\mathfrak{g}) ;$ où $\mathfrak{l}=\operatorname{End}\left(X^{\prime}\right)+\mathfrak{g}(U)$ ( $L$ le groupe correspondant $)$ et

$$
\psi_{\mathfrak{n}}^{K}(x)=\int_{\mathfrak{n}, K} \psi(k \cdot(x+n)) d n d k .
$$

On pose $G^{\prime}\left(V_{c}^{\prime}\right)$ le groupe des isométries associé à $\left(V_{c}^{\prime},(,)^{\prime}\right)$; on note $\mathfrak{g}^{\prime}\left(V_{c}^{\prime}\right)$ son algèbre de Lie. La paire de groupes $\left(G(U), G^{\prime}\left(V_{c}^{\prime}\right)\right)$ est une paire duale réductive qui est réalisée dans le groupe symplectique $S p\left(W_{1}\right)$ avec $W_{1}=\operatorname{Hom}_{\mathbb{C}}\left(V_{c}^{\prime}, U\right)$. On note l'intégrale de Cauchy Harish-Chandra associée à cette paire $\mathbf{C h c}^{U, V_{c}^{\prime}}$. On remarque ensuite que la restriction de la sous-algèbre de Cartan $\mathfrak{h}^{\prime}$ à $V_{c}^{\prime}$ est une sous-algèbre de Cartan compacte de $\mathfrak{g}^{\prime}\left(V_{c}^{\prime}\right)$. Le théorème suivant fournit un lien entre Chc et Chc $^{U, V_{c}^{\prime}}$. 
ThÉorème 2.2.7 ([6, Proposition 11.14]). - Pour $\mathfrak{h}^{\prime} \in \operatorname{Car}\left(\mathfrak{g}^{\prime}\right)$, il existe une constante $\mathfrak{C}_{\mathfrak{g}, \mathfrak{g}^{\prime}, \mathfrak{h}^{\prime}}$ telle que pour $x^{\prime} \in \mathfrak{h}^{\text {reg }}$ et $\psi \in \mathcal{D}(\mathfrak{g})$, on a :

$\operatorname{Chc}(\psi)\left(x^{\prime}\right)=\frac{\mathfrak{C}_{\mathfrak{g}, \mathfrak{g}^{\prime}, \mathfrak{h}^{\prime}}}{\left|\operatorname{det}\left(\left.\operatorname{ad}\left(x^{\prime}\right)\right|_{\mathfrak{n}^{\prime}}\right)\right|} \mathbf{C h c}^{U, V_{c}^{\prime}}\left(\int_{G L\left(X^{\prime}\right) / H_{s}^{\prime}} \psi_{\mathfrak{n}}^{K}\left(g x_{s}^{\prime} g^{-1}+.\right) d \dot{g}\right)\left(x_{c}^{\prime}\right)$, où $H_{s}^{\prime}=H^{\prime} \mid X^{\prime}$ et $x_{c}^{\prime}=x^{\prime} \mid V_{c}^{\prime}$ et $x_{s}^{\prime}=x^{\prime} \mid X^{\prime}$.

REMARQUE

(1) On a $\mathfrak{C}_{\mathfrak{g}, \mathfrak{g}^{\prime}, \mathfrak{h}^{\prime}}=\mathfrak{C}_{\mathfrak{g}, \mathfrak{g}^{\prime}, \mathfrak{h}_{1}^{\prime}}$ si $\mathfrak{h}^{\prime}$ et $\mathfrak{h}_{1}^{\prime}$ sont conjuguées.

(2) $\mathfrak{C}_{\mathfrak{g}, \mathfrak{g}^{\prime}, \mathfrak{h}_{\varnothing}^{\prime}}=1$. 


\section{CHAPITRE 3}

\section{PROPRIÉTÉS DE Chc SI $G$ ET $G^{\prime}$ SONT DE RANG 2}

Dans ce chapitre, on étudie les propriétés de Chc pour les paires

$$
\left(G, G^{\prime}\right) \in\{(U(2), U(2)),(U(2), U(1,1)),(U(1,1), U(2)),(U(1,1), U(1,1))\} .
$$

On rappelle dans la première section les propriétés des intégrales invariantes de HarishChandra pour $\mathfrak{g}=\mathfrak{u}(2)$ et $\mathfrak{u}(1,1)$. Dans la section suivante, on obtient les propriétés de $\operatorname{Chc}(\psi)$ si $\mathfrak{g}=\mathfrak{u}(2)$. Puis dans la section 3 , on calcule des développements asymptotiques d'intégrales qui permettent dans la sous-section 4 d'obtenir les propriétés si $\mathfrak{g}=\mathfrak{u}(1,1)$. Dans la sous-section 5 , on rassemble toutes ces propriétés locales.

\subsection{Préliminaires}

On adopte les notations suivantes dans cette partie. On pose $\kappa_{\mathfrak{g}}=\kappa_{\mathfrak{g}^{\prime}}=\operatorname{Tr}_{\mathbb{R}}(x y)$ pour $x, y \in \mathfrak{g l}_{2}(\mathbb{C})$. On note

$$
\begin{array}{ll}
\mathfrak{h}_{\varnothing}=\left\{\left(\begin{array}{cc}
\mathrm{i} x_{1} & 0 \\
0 & \mathrm{i} x_{2}
\end{array}\right) \mid x_{1}, x_{2} \in \mathbb{R}\right\} ; \mathfrak{h}_{\varnothing}^{\prime}=\left\{\left(\begin{array}{cc}
\mathrm{i} x_{1}^{\prime} & 0 \\
0 & \mathrm{i} x_{2}^{\prime}
\end{array}\right) \mid x_{1}^{\prime}, x_{2}^{\prime} \in \mathbb{R}\right\} \\
\mathfrak{h}_{\mathcal{S}}=\left\{\left(\begin{array}{cc}
\mathrm{i} v & u \\
u & \mathrm{i} v
\end{array}\right) \mid u, v \in \mathbb{R}\right\} ; & \mathfrak{h}_{\mathcal{S}}^{\prime}=\left\{\left(\begin{array}{cc}
\mathrm{i} v^{\prime} & u^{\prime} \\
u^{\prime} & \mathrm{i} v^{\prime}
\end{array}\right) \mid u^{\prime}, v^{\prime} \in \mathbb{R}\right\} .
\end{array}
$$

On pose $\alpha=\left(x_{2}^{\prime}-x_{1}^{\prime}\right) / 2$ et $\beta=\left(x_{2}^{\prime}+x_{1}^{\prime}\right) / 2$. On désigne par $(1,2)$ la transposition et par $i d$ l'élément neutre de $\mathfrak{S}(\{1,2\})$.

3.1.1. Intégrales invariantes de Harish-Chandra. — Soit $\psi \in \mathcal{D}(\mathfrak{g})$, les intégrales invariantes de $\psi$ sont définies ainsi : Si $\mathfrak{g}=\mathfrak{u}(2)$ ou $\mathfrak{u}(1,1)$, on pose

$$
\begin{aligned}
\psi_{\varnothing}: \mathfrak{h}_{\varnothing}^{\mathrm{reg}} & \longrightarrow \mathbb{C} \\
x & \longmapsto \mathrm{i}\left(x_{2}-x_{1}\right) \int_{G / H_{\varnothing}} \psi(g \cdot x) d \dot{g} .
\end{aligned}
$$

Si $\mathfrak{g}=\mathfrak{u}(1,1)$, on pose

$$
\begin{aligned}
\psi_{\mathcal{S}}: \mathfrak{h}_{\mathcal{S}}^{\mathrm{reg}} & \longrightarrow \mathbb{C} \\
x & \longmapsto 2|u| \int_{G / H_{\mathcal{S}}} \psi(g \cdot x) d \dot{g}
\end{aligned}
$$


Si $\mathfrak{g}=\mathfrak{u}(1,1)$, on considère la transformée de Cayley définie ainsi

$$
c_{\mathcal{S}}\left(\left(\begin{array}{cc}
\mathrm{i}(\beta+\alpha) & 0 \\
0 & \mathrm{i}(\beta-\alpha)
\end{array}\right)\right)=\left(\begin{array}{ll}
\mathrm{i} \beta & \mathrm{i} \alpha \\
\mathrm{i} \alpha & \mathrm{i} \beta
\end{array}\right) .
$$

Cette application induit un isomorphisme d'algèbres entre $\operatorname{Sym}\left(\mathfrak{h}_{\varnothing, \mathbb{C}}\right)$ et $\operatorname{Sym}\left(\mathfrak{h}_{\mathcal{S}, \mathbb{C}}\right)$ que l'on note aussi $c_{\mathcal{S}}$. Pour toute fonction $\theta$ définie sur $\mathfrak{h}_{\varnothing}^{\text {reg }}$ ou $\mathfrak{h}_{\varnothing}^{\text {reg }}$ telle que les limites

$$
\lim _{\substack{t \rightarrow 0 \\ t>0}} \theta(v+t, v-t) \text { et } \lim _{\substack{t \rightarrow 0 \\ t<0}} \theta(v+t, v-t)
$$

ont un sens, on notera

$$
\langle\theta\rangle(v)=\lim _{\substack{t \rightarrow 0 \\ t>0}} \theta(v+t, v-t)-\lim _{\substack{t \rightarrow 0 \\ t<0}} \theta(v+t, v-t) .
$$

Les fonctions $\psi_{\varnothing}$ et $\psi_{\mathcal{S}}$ ont les propriétés suivantes :

THÉORÈmE 3.1.1. - Si $\mathfrak{g}=\mathfrak{u}(2)$, la fonction $\psi_{\varnothing}$ se prolonge en une fonction lisse sur $\mathfrak{h}_{\varnothing}$ à support compact et vérifie la relation

$$
\psi_{\varnothing}\left(x_{1}, x_{2}\right)=-\psi_{\varnothing}\left(x_{2}, x_{1}\right)
$$

pour $x \in \mathfrak{h}_{\varnothing}$.

Pour $\mathfrak{g}=\mathfrak{u}(2)$ ou $\mathfrak{u}(1,1)$, le centre de $\mathfrak{g}$ s'identifie à $\mathbb{R}$ par l'application

$$
\begin{aligned}
& \mathbb{R} \longrightarrow \mathfrak{g} \\
& v \longmapsto\left(\begin{array}{cc}
\mathbf{i} v & 0 \\
0 & \mathbf{i} v
\end{array}\right) .
\end{aligned}
$$

THÉORÈme 3.1.2. - Si $\mathfrak{g}=\mathfrak{u}(1,1)$,

(1) La fonction $\psi_{\mathcal{S}}$ se prolonge en une fonction lisse à support compact sur $\mathfrak{h}_{\mathcal{S}}$ et vérifie la relation

$$
\psi_{\mathcal{S}}(u, v)=\psi_{\mathcal{S}}(-u, v)
$$

pour $x \in \mathfrak{h}_{\mathcal{S}}$.

(2) La fonction $\psi_{\varnothing}$ est lisse sur $\mathfrak{h}_{\varnothing}^{\mathrm{reg}}$ et à support borné.

(3) Pour tout $w \in \operatorname{Sym}\left(\mathfrak{h}_{\varnothing, \mathbb{C}}\right)$, la fonction $\partial(w) \psi_{\varnothing}$ se prolonge par continuité sur les ensembles $\left\{x \in \mathfrak{h}_{\varnothing} \mid x_{1} \leqslant x_{2}\right\}$ et $\left\{x \in \mathfrak{h}_{\varnothing} \mid x_{1} \geqslant x_{2}\right\}$. On peut donc considérer $\left\langle\partial(w) \psi_{\varnothing}\right\rangle$.

(4) On a la relation de saut

$$
\left\langle\partial(w) \psi_{\varnothing}\right\rangle(v)=\mathrm{i} \partial\left(c_{\mathcal{S}}(w)\right) \psi_{\mathcal{S}}(0, v) .
$$

REMARQUE. - Soit $s$ la réflexion de $\mathfrak{h}_{\varnothing, \mathbb{C}}$ définie par

$$
s\left(\left(\begin{array}{ll}
a & 0 \\
0 & b
\end{array}\right)\right)=\left(\begin{array}{ll}
b & 0 \\
0 & a
\end{array}\right) .
$$


L'application $s$ induit un automorphisme de l'algèbre symétrique $\operatorname{Sym}\left(\mathfrak{h}_{\varnothing, \mathbb{C}}\right)$ que l'on note aussi $s$. Pour $w \in \operatorname{Sym}\left(\mathfrak{h}_{\varnothing, \mathbb{C}}\right)$, si $s(w)=-w$, on a $\left\langle\partial(w) \psi_{\varnothing}\right\rangle=0$ et la fonction $\partial(w) \psi_{\varnothing}$ se prolonge en une fonction continue sur $\mathfrak{h}_{\varnothing}$.

3.1.2. Fonctions associées à Chc. - Soit $\psi \in \mathcal{D}(\mathfrak{g})$. On associe à la fonction $\operatorname{Chc}(\psi)$ les fonctions suivantes.

Si $\mathfrak{g}^{\prime}=\mathfrak{u}(1,1)$ ou $\mathfrak{u}(2)$, on pose

$$
\begin{aligned}
\operatorname{Chc}(\psi)_{\varnothing}: \mathfrak{h}_{\varnothing}^{\text {reg }} & \longrightarrow \mathbb{C} \\
x^{\prime} & \longmapsto \mathrm{i}\left(x_{2}^{\prime}-x_{1}^{\prime}\right) \mathbf{C h c}(\psi)\left(x^{\prime}\right) .
\end{aligned}
$$

Si $\mathfrak{g}^{\prime}=\mathfrak{u}(1,1)$, on pose

$$
\begin{aligned}
\operatorname{Chc}(\psi)_{\mathcal{S}}: \mathfrak{h}_{\mathcal{S}}^{\prime \text { reg }} & \longrightarrow \mathbb{C} \\
x^{\prime} & \longmapsto 2\left|u^{\prime}\right| \mathbf{C h c}(\psi)\left(x^{\prime}\right) .
\end{aligned}
$$

\subsection{Propriétés de Chc pour $G$ compact}

L'espace $V$ est un espace hermitien de signature ${ }^{(1)}(0,2)(\mathbf{p}=0, \mathbf{q}=2)$. On a ainsi $\operatorname{sign}\left(V_{1}\right)=\operatorname{sign}\left(V_{2}\right)=-1$.

3.2.1. Pour $G^{\prime}=U(2)$. — Dans cette sous-section, par un calcul explicite, on obtient le corollaire 3.2.2 qui précise les propriétés locales de Chc.

Le corollaire 2.2.5 permet d'obtenir :

Proposition 3.2.1. - Pour $\psi \in \mathcal{D}(\mathfrak{g})$ et $x^{\prime} \in \mathfrak{h}_{\varnothing}^{\text {reg }}$, on $a:$

$$
\begin{aligned}
& \mathbf{C h c}(\psi)_{\varnothing}\left(x^{\prime}\right)=-16 \int_{\mathbb{R}^{2}} \ln \left|x_{1}-x_{1}^{\prime}\right| \ln \left|x_{2}-x_{2}^{\prime}\right| \partial_{1,2} \psi_{\varnothing}(x) d x \\
&-16 \cdot \mathrm{i} \pi\left\{\int_{\mathbb{R}} \partial_{2} \psi_{\varnothing}\left(x_{1}^{\prime}, x_{2}\right) \ln \left|x_{2}-x_{2}^{\prime}\right| d x_{2}+\int_{\mathbb{R}} \partial_{1} \psi_{\varnothing}\left(x_{1}, x_{2}^{\prime}\right) \ln \left|x_{1}-x_{1}^{\prime}\right| d x_{1}\right\} \\
&+16 \pi^{2} \psi_{\varnothing}\left(x_{1}^{\prime}, x_{2}^{\prime}\right) .
\end{aligned}
$$

Démonstration. - Avec les notations de la section 2.2, on a pour $L=i d$ ou $(1,2)$

$$
y_{L(k)}^{L}=\operatorname{sign}\left(V_{k}^{\prime}\right) \operatorname{sign}\left(V_{L(k)}\right) \text { avec } k=1,2
$$

ainsi

$$
y^{L}=\left(\begin{array}{l}
1 \\
1
\end{array}\right)
$$

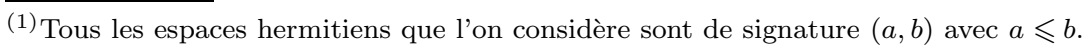


Ensuite, on a $P_{L, 0}=-\varepsilon(L)$ d'après le corollaire $2.2 .2, n_{\varnothing}=\frac{1}{2}$ (p. 15) et $C_{\mathfrak{g}, \mathfrak{g}^{\prime}}=16$ (p. 15); d'où :

$$
\begin{aligned}
& \operatorname{Chc}(\psi)_{\varnothing}\left(x^{\prime}\right)=\overline{\pi_{\mathfrak{h}_{\varnothing}^{\prime}}}\left(x^{\prime}\right) \mathbf{C h c}(\psi)\left(x^{\prime}\right) \\
& =\lim _{\substack{t \rightarrow 0 \\
t>0}}\left\{-8 \cdot \int_{\mathbb{R}^{2}} \frac{\psi_{\varnothing}(x)}{\left(x_{1}+\mathrm{i} t-x_{1}^{\prime}\right)\left(x_{2}+\mathrm{i} t-x_{2}^{\prime}\right)} d x\right. \\
& \left.\quad+8 \cdot \int_{\mathbb{R}^{2}} \frac{\psi_{\varnothing}(x)}{\left(x_{2}+\mathrm{i} t-x_{1}^{\prime}\right)\left(x_{1}+\mathrm{i} t-x_{2}^{\prime}\right)} d x\right\} .
\end{aligned}
$$

Comme $\psi_{\varnothing}$ est une fonction antisymétrique, c'est à dire vérifie

$$
\psi_{\varnothing}\left(x_{1}, x_{2}\right)=-\psi_{\varnothing}\left(x_{2}, x_{1}\right)
$$

on a l'égalité

$$
\int_{\mathbb{R}^{2}} \frac{\psi_{\varnothing}(x)}{\left(x_{2}+\mathrm{i} t-x_{1}^{\prime}\right)\left(x_{1}+\mathrm{i} t-x_{2}^{\prime}\right)} d x=-\int_{\mathbb{R}^{2}} \frac{\psi_{\varnothing}(x)}{\left(x_{2}+\mathrm{i} t-x_{2}^{\prime}\right)\left(x_{1}+\mathrm{i} t-x_{1}^{\prime}\right)} d x .
$$

ainsi, l'égalité (3.2.1) s'écrit

$$
\operatorname{Chc}(\psi)_{\varnothing}\left(x^{\prime}\right)=\lim _{\substack{t \rightarrow 0 \\ t>0}}-16 \int_{\mathbb{R}^{2}} \frac{\psi_{\varnothing}(x)}{\left(x_{1}+\mathrm{i} t-x_{1}^{\prime}\right)\left(x_{2}+\mathrm{i} t-x_{2}^{\prime}\right)} d x ;
$$

puis en intégrant par parties par rapport à $x_{1}$ et $x_{2}$, l'expression précédente devient

$$
\begin{aligned}
&- 16 \int_{\mathbb{R}^{2}} \ln \left|x_{1}-x_{1}^{\prime}\right| \ln \left|x_{2}-x_{2}^{\prime}\right| \partial_{1,2} \psi_{\varnothing}(x) d x \\
&-16 \cdot \mathrm{i} \pi\left\{\int_{\mathbb{R}} \partial_{2} \psi_{\varnothing}\left(x_{1}^{\prime}, x_{2}\right) \ln \left|x_{2}-x_{2}^{\prime}\right| d x_{2}+\int_{\mathbb{R}} \partial_{1} \psi_{\varnothing}\left(x_{1}, x_{2}^{\prime}\right) \ln \left|x_{1}-x_{1}^{\prime}\right| d x_{1}\right\} \\
&+16 \pi^{2} \psi_{\varnothing}\left(x_{1}^{\prime}, x_{2}^{\prime}\right) .
\end{aligned}
$$

Cette proposition permet d'obtenir le résultat suivant :

Corollaire 3.2.2. - La fonction

$$
\operatorname{Chc}(\psi)_{\varnothing}
$$

se prolonge en une fonction lisse sur $\mathfrak{h}_{\varnothing}^{\prime}$.

3.2.2. Pour $G^{\prime}=U(1,1)$. — De nouveau par un calcul explicite, on obtient les propriétés de Chc dans la proposition suivante et dans le corollaire 3.2.4.

Proposition 3.2.3. - Pour $\psi \in \mathcal{D}(\mathfrak{g})$, on a

(1) Si $x^{\prime} \in \mathfrak{h}_{\mathcal{S}}^{\text {/reg }}$, on a $\operatorname{Chc}(\psi)\left(x^{\prime}\right)=0$. 
(2) Si $x^{\prime} \in \mathfrak{h}_{\varnothing}^{\text {reg }}$, on $a$ :

$$
\begin{array}{r}
\operatorname{Chc}(\psi)_{\varnothing}\left(x^{\prime}\right)=-16 \int_{\mathbb{R}^{2}} \ln \left|x_{1}-x_{1}^{\prime}\right| \ln \left|x_{2}-x_{2}^{\prime}\right| \partial_{1,2} \psi_{\varnothing}(x) d x \\
+16 \mathbf{i} \pi \int_{\mathbb{R}} \ln \left|u-x_{2}^{\prime}\right| \partial_{2} \psi_{\varnothing}\left(x_{1}^{\prime}, u\right) d u-16 \mathbf{i} \pi \int_{\mathbb{R}} \ln \left|u-x_{1}^{\prime}\right| \partial_{1} \psi_{\varnothing}\left(u, x_{2}^{\prime}\right) d u \\
\quad-16 \pi^{2} \psi_{\varnothing}\left(x_{1}^{\prime}, x_{2}^{\prime}\right) .
\end{array}
$$

Démonstration. — Le point (1) découle de la proposition 2.2.6. Pour le point (2), on a

$$
\operatorname{sign}\left(V_{1}\right)=\operatorname{sign}\left(V_{2}\right)=\operatorname{sign}\left(V_{2}^{\prime}\right)=-1 \text { et } \operatorname{sign}\left(V_{1}^{\prime}\right)=1
$$

ainsi

$$
y^{i d}=\left(\begin{array}{c}
-1 \\
1
\end{array}\right) \quad y^{(1,2)}=\left(\begin{array}{c}
1 \\
-1
\end{array}\right) .
$$

D'après le corollaire 2.2.5, on obtient

$$
\begin{aligned}
& \operatorname{Chc}(\psi)_{\varnothing}\left(x^{\prime}\right) \\
& =\lim _{\substack{t \rightarrow 0 \\
t>0}}\left\{-8 \int_{\mathbb{R}^{2}} \frac{\psi_{\varnothing}(x)}{\left(x_{1}-\mathrm{i} t-x_{1}^{\prime}\right)\left(x_{2}+\mathrm{i} t-x_{2}^{\prime}\right)} d x\right. \\
& \left.+8 \int_{\mathbb{R}^{2}} \frac{\psi_{\varnothing}(x)}{\left(x_{2}-\mathrm{i} t-x_{1}^{\prime}\right)\left(x_{1}+\mathrm{i} t-x_{2}^{\prime}\right)} d x\right\} .
\end{aligned}
$$

Comme $\psi_{\varnothing}$ est antisymétrique, cette expression s'écrit :

$$
-16 \lim _{\substack{t \rightarrow 0 \\ t>0}} \int_{\mathbb{R}^{2}} \frac{\psi_{\varnothing}(x)}{\left(x_{1}-\mathrm{i} t-x_{1}^{\prime}\right)\left(x_{2}+\mathrm{i} t-x_{2}^{\prime}\right)} d x .
$$

Puis en intégrant par parties par rapport à $x_{1}$ et $x_{2}$, on obtient :

$$
\begin{gathered}
-16 \int_{\mathbb{R}^{2}} \ln \left|x_{1}-x_{1}^{\prime}\right| \ln \left|x_{2}-x_{2}^{\prime}\right| \partial_{1,2} \psi_{\varnothing}(x) d x+16 \mathbf{i} \pi \int_{\mathbb{R}} \ln \left|x_{2}-x_{2}^{\prime}\right| \partial_{2} \psi_{\varnothing}\left(x_{1}^{\prime}, x_{2}\right) d x_{2} \\
-16 \mathbf{i} \pi \int_{\mathbb{R}} \ln \left|x_{1}-x_{1}^{\prime}\right| \partial_{1} \psi_{\varnothing}\left(x_{1}, x_{2}^{\prime}\right) d x_{1}-16 \pi^{2} \psi_{\varnothing}\left(x_{1}^{\prime}, x_{2}^{\prime}\right) .
\end{gathered}
$$

Corollaire 3.2.4. - La fonction

\section{$\operatorname{Chc}(\psi)_{\varnothing}$}

se prolonge en une fonction lisse sur $\mathfrak{h}_{\varnothing}^{\prime}$.

\section{3. Études de quelques intégrales}

Le calcul de $\operatorname{Chc}(\psi)_{\varnothing}$ dans le cas où $G=U(1,1)$ (section 4) nécessitent l'étude de quelques intégrales, c'est l'objet de cette section. Dans la première sous-section, on s'intéresse à une intégrale qui intervient dans la contribution de $\psi_{\mathcal{S}}$. On utilise le lemme 3.3.2 dans la sous-section 4.1. Dans la deuxième sous-section, on s'occupe de 
certaines intégrales qui apparaissent dans l'étude des termes dépendant de $\psi_{\varnothing}$. Ces résultats sont utilisés dans la section suivante.

3.3.1. Étude d'une intégrale liée à la partie déployée. — On désigne par Arg l'argument principal (i.e. défini sur $\mathbb{C} \backslash \mathbb{R}_{-}$) et $P_{+}$le demi-plan de Poincaré.

DÉfinition 3.3.1. - Pour une fonction $\phi \in \mathcal{C}_{c}^{1}(\mathbb{C})$, on pose :

$$
\Xi_{\phi}\left(x_{1}^{\prime}, x_{2}^{\prime}\right)=\int_{\left.\mathbb{R}_{+} \times\right] 0, \pi[}\left(\cos (\theta) \partial_{1} \phi+\sin (\theta) \partial_{2} \phi\right)\left(\rho e^{\mathrm{i} \theta}+x_{1}^{\prime}\right) \operatorname{Arg}\left(\rho e^{\mathrm{i} \theta}+x_{1}^{\prime}-x_{2}^{\prime}\right) d \rho d \theta
$$

et $\widehat{\Xi}_{\phi}\left(x_{1}^{\prime}, x_{2}^{\prime}\right)=\Xi_{\phi}\left(x_{1}^{\prime}, x_{2}^{\prime}\right)-\Xi_{\phi}\left(x_{2}^{\prime}, x_{1}^{\prime}\right)$.

Pour une fonction $\phi \in \mathcal{C}_{c}^{0}(\mathbb{C})$ et $\beta \in \mathbb{R}$, on note $\phi(\beta+$.) la fonction définie par

$$
\phi(\beta+.)(v)=\phi(\beta+v) .
$$

On désigne par $d$ un élément de $\mathbb{N}$. L'objectif de cette sous-section est de montrer le résultat suivant :

Proposition 3.3.2. - Soit $\phi \in \mathcal{C}_{c}^{d+2}(\mathbb{C})$ telle que :

$$
\phi(z)=\phi(\bar{z}) \quad \forall z \in \mathbb{C}
$$

alors $\widehat{\Xi}_{\phi} \in \mathcal{C}^{d}\left(\mathbb{R}^{2}\right)$. Pour démontrer cette proposition (p. 34), on aura besoin de trois lemmes qui nécessitent l'introduction des notations suivantes. Pour $\phi \in \mathcal{C}_{c}^{1}(\mathbb{C})$, on pose

$$
\begin{gathered}
D \phi=\partial_{1} \phi+\mathrm{i} \partial_{2} \phi \\
\widetilde{\Xi}_{\phi}(\alpha)=\int_{P_{+}} \frac{\operatorname{Arg}(z-2 \alpha)}{z} D \phi(z-\alpha) d z \quad(\alpha \neq 0)
\end{gathered}
$$

et

$$
\Omega_{\phi}(\alpha)=\int_{P_{+}} \frac{\operatorname{Arg}(w)}{w+2 \varepsilon} D \phi(|\alpha| w+\alpha) d w \quad(\alpha \neq 0)
$$

où $\varepsilon=\operatorname{sign}(\alpha)$.

REMARQUE. - Si $\phi$ est à valeurs réelles, on a la relation

$$
\Re\left(e^{-\mathrm{i} \theta} D \phi\right)=\cos (\theta) \partial_{1} \phi+\sin (\theta) \partial_{2} \phi=\partial_{\rho}\left(\phi\left(\rho e^{\mathrm{i} \theta}\right)\right) .
$$

Les égalités (3.3.2) et (3.3.20) (p.35) précisent le lien entre $\widetilde{\Xi}_{\phi}$ et $\Omega_{\phi}$ d'une part et $\Xi_{\phi}$ et $\widetilde{\Xi}_{\phi}$ d'autre part.

LEMme 3.3.3. - Soit $\phi \in \mathcal{C}_{c}^{d+1}(\mathbb{C})$. Les fonctions

$$
\begin{aligned}
\{\alpha \neq 0\} & \subset \mathbb{R}^{2} \longrightarrow \mathbb{C} \\
(\alpha, \beta) & \longmapsto \widetilde{\Xi}_{\phi(\beta+.)}(\alpha) \\
(\alpha, \beta) & \longmapsto \Omega_{\phi(\beta+.)}(\alpha)
\end{aligned}
$$

appartiennent à $\mathcal{C}^{d}(\{\alpha \neq 0\})$. 
Démonstration. - On a

$$
\Omega_{\phi(\beta+.)}(\alpha)=\int_{P_{+}} \frac{\operatorname{Arg}(w)}{w+2 \varepsilon} D \phi(|\alpha| w+\alpha+\beta) d w,
$$

ainsi, on peut dériver sous le signe somme et cette fonction est de classe $C^{d}$ sur l'ouvert $\{\alpha \neq 0\} \subset \mathbb{R}^{2}$. Ensuite, avec le changement de variable $z=|\alpha| w+2 \alpha$, on obtient la relation

$$
\widetilde{\Xi}_{\phi}(\alpha)=|\alpha| \Omega_{\phi}(\alpha)
$$

ainsi, on a

$$
\widetilde{\Xi}_{\phi(\beta+.)}(\alpha)=|\alpha| \Omega_{\phi(\beta+.)}(\alpha) .
$$

On en déduit le résultat pour la première fonction.

Lemme 3.3.4. - Pour $\phi \in \mathcal{C}_{c}^{1}(\mathbb{C})$ impaire telle que

$$
\phi(z)=\phi(\bar{z}) \forall z \in \mathbb{C}
$$

on $a$

$$
\widehat{\Xi}_{\phi}(-\alpha, \alpha)=\pi^{2} \phi(\alpha) \text {. }
$$

Démonstration. - Il suffit de démontrer ce résultat dans le cas où $\phi$ est à valeurs réelles. Soit $\phi \in \mathcal{C}_{c}^{1}(\mathbb{C})$ vérifiant cette hypothèse. Par définition, on a la relation

$$
\phi(z)=-\phi(-z) \forall z \in \mathbb{C}
$$

qui permet d'obtenir

$$
D \phi(-z)=-D \phi(z)
$$

De plus grâce à (3.3.3), on a

$$
D \phi(\bar{z})=\overline{D \phi(z)}
$$

ainsi, on a

$$
D \phi(z)=\overline{D \phi(-\bar{z})} .
$$

En considérant le changement de variables $z \mapsto-\bar{z}$, on obtient les égalités

$$
\begin{aligned}
& \widetilde{\Xi}_{\phi}(\alpha)=-\int_{P_{+}} \frac{\operatorname{Arg}(-\bar{z}-2 \alpha)}{\bar{z}} D \phi(-\bar{z}-\alpha) d z \\
& =\int_{P_{+}} \frac{\operatorname{Arg}(z+2 \alpha)}{\bar{z}} \overline{D \phi(z+\alpha)} d z-\pi \int_{P_{+}} \frac{\overline{D \phi(z+\alpha)}}{\bar{z}} d z \\
& =\overline{\widetilde{\Xi}_{\phi}(-\alpha)}-\pi \int_{P_{+}} \frac{\overline{D \phi(z+\alpha)}}{\bar{z}} d z \text {. }
\end{aligned}
$$


Puis, on a en posant $z=\rho e^{\mathrm{i} \theta}$

$$
\begin{aligned}
\widehat{\Xi}_{\phi}(-\alpha, \alpha) & =-\pi \int_{P_{+}} \Re\left\{\frac{\overline{D \phi(z+\alpha)}}{\bar{z}}\right\} d z \\
& =-\pi \int_{\left.\mathbb{R}_{+} \times\right] 0, \pi[} \Re\left\{e^{\mathrm{i} \theta \overline{D \phi\left(\rho e^{\mathrm{i} \theta}+\alpha\right)}}\right\} d \rho d \theta \\
& =-\pi \int_{\left.\mathbb{R}_{+} \times\right] 0, \pi[} \partial_{\rho} \phi\left(\rho e^{\mathrm{i} \theta}+\alpha\right) d \rho d \theta=\pi^{2} \phi(\alpha) .
\end{aligned}
$$

LEMME 3.3.5. - Soit $\phi \in \mathcal{C}_{c}^{d+1}(\mathbb{C})$ paire ${ }^{(2)}$ avec d pair telle que

$$
\phi(z)=\phi(\bar{z}) \forall z \in \mathbb{C}
$$

alors la fonction

$$
\alpha \in \mathbb{R}^{*} \longmapsto \widehat{\Xi}_{\phi}(-\alpha, \alpha)
$$

appartient à $\mathcal{C}^{d}(\mathbb{R})$. De plus, pour toute partie compacte $\mathcal{K}$ de $\mathbb{C}, n \leqslant d$ pair, il existe une famille finie d'éléments $w_{1}, \ldots, w_{m}$ de Sym $(\mathbb{C})$ de degrés inférieurs à $n+1$ telle que pour $\varepsilon>0$, il existe $\eta>0$ vérifiant

$$
\left|\left(\widehat{\Xi}_{\phi}(-\alpha, \alpha)\right)^{(n)}\right| \leqslant \varepsilon \sup _{\substack{x \in \mathbb{C} \\ 1 \leqslant i \leqslant m}}\left|\partial\left(w_{i}\right) \phi(x)\right|
$$

dès que $|\alpha| \leqslant \eta$ pour $\phi \in \mathcal{C}_{\mathcal{K}}^{d+1}(\mathbb{C})$.

REmarque. - Cette inégalité exprime que $\left(\widehat{\Xi}_{\phi}(-\alpha, \alpha)\right)^{(n)}$ converge vers 0 si $\alpha$ tend vers 0 uniformément en $\phi$.

Démonstration. - Il suffit de montrer le résultat pour $\phi$ à valeurs réelles. On suppose $\phi$ à valeurs réelles. Comme $\phi$ est une fonction paire sur $\mathbb{C}$, les deux égalités :

$$
\phi(z)=\phi(\bar{z}) \text { et } \phi(z)=\phi(-z)
$$

impliquent

$$
\partial_{1,2}^{p, q} \phi(0)=0
$$

dès que $p$ ou $q$ est impair. Le changement de variable $z=|\alpha| w+2 \alpha$ permet d'exprimer la fonction $\widetilde{\Xi}_{\phi}$ ainsi :

$$
|\alpha| \int_{P_{+}} \frac{\operatorname{Arg}(w)}{w+2 \varepsilon} D \phi(|\alpha| w+\alpha) d w=|\alpha| \Omega_{\phi}(\alpha)
$$

où $\varepsilon=\operatorname{sign}(\alpha)$. Pour $n \leqslant d$, on a :

$$
\widetilde{\Xi}_{\phi}^{(n)}(\alpha)=|\alpha| \Omega_{\phi}^{(n)}(\alpha)+n \varepsilon \Omega_{\phi}^{(n-1)}(\alpha) .
$$

$\overline{(2)}$ i.e. $\phi(z)=\phi(-z)$ pour $z \in \mathbb{C}$. 
La dérivée d'ordre $n$ de $\Omega_{\phi}(\alpha)$ en $\alpha(\alpha \neq 0)$ est égale à :

$$
\sum_{p=0}^{n} \mathcal{C}_{n}^{p} \int_{P_{+}} \frac{\operatorname{Arg}(w)}{w+2 \varepsilon}\left(\varepsilon w_{1}+1\right)^{p}\left(\varepsilon w_{2}\right)^{n-p} \partial_{1,2}^{p, n-p}(D \phi)(|\alpha| w+\alpha) d w
$$

où $w_{1}$ (resp. $w_{2}$ ) désigne la partie réelle (resp. la partie imaginaire) de $w$. On pose maintenant : $u=|\alpha| w ;$ l'expression précédente devient :

$$
\frac{\varepsilon}{\alpha^{n+1}} \sum_{p=0}^{n} \mathcal{C}_{n}^{p} \int_{P_{+}} \frac{\operatorname{Arg}(u)}{u+2 \alpha}\left(u_{1}+\alpha\right)^{p} u_{2}^{n-p} \partial_{1,2}^{p, n-p}(D \phi)(u+\alpha) d u .
$$

En développant les polynômes en $u_{1}$, on obtient :

$$
\varepsilon \sum_{p=0}^{n} \sum_{r=0}^{p} \frac{\mathcal{C}_{n}^{p} \mathcal{C}_{p}^{r}}{\alpha^{n+1+r-p}} \int_{P_{+}} \frac{\operatorname{Arg}(u)}{u+2 \alpha} u_{1}^{r} u_{2}^{n-p} \partial_{1,2}^{p, n-p}(D \phi)(u+\alpha) d u .
$$

Jusqu'à la fin de cette preuve, $n$ désigne un entier pair. Dans un premier temps, on détermine le développement asymptotique de

$$
\alpha \longmapsto|\alpha| \Omega_{\phi}^{(n)}(\alpha)
$$

en 0 puis on s'intéresse (p. 31) à

$$
\alpha \longmapsto \varepsilon \Omega_{\phi}^{(n-1)}(\alpha)
$$

D'après (3.3.5), on a l'égalité :

$$
|\alpha| \Omega_{\phi}^{(n)}(\alpha)=\sum_{p=0}^{n} \sum_{r=0}^{p} \frac{\mathcal{C}_{n}^{p} \mathcal{C}_{p}^{r}}{\alpha^{n+r-p}} \int_{P_{+}} \frac{\operatorname{Arg}(u)}{u+2 \alpha} u_{1}^{r} u_{2}^{n-p} \partial_{1,2}^{p, n-p}(D \phi)(u+\alpha) d u .
$$

On a la relation si $u \notin\{0,-2 \alpha\}$

$$
\frac{1}{u+2 \alpha}=\sum_{k=0}^{n+r-p}(-1)^{k} \frac{2^{k} \alpha^{k}}{u^{k+1}}+\left(-\frac{2 \alpha}{u}\right)^{n+r-p+1} \frac{1}{u+2 \alpha}
$$

En remplaçant $\frac{1}{u+2 \alpha}$ dans l'expression (3.3.6), il apparaît l'expression

$$
(-2)^{n+r-p+1} \alpha \int_{P_{+}} \frac{\operatorname{Arg}(u)}{u+2 \alpha} \frac{u_{1}^{r} u_{2}^{n-p}}{u^{n+r-p+1}} \partial_{1,2}^{p, n-p}(D \phi)(u+\alpha) d u
$$

Montrons que cette expression tend vers 0 si $\alpha$ tend vers 0 uniformément en $\phi$. Soit $\mathcal{K}$ une partie compacte de $\mathbb{C}$ et $\phi \in \mathcal{C}_{\mathcal{K}}^{n+1}(\mathbb{C})$, on a l'inégalité

$$
\begin{aligned}
& \left|(-2)^{n+r-p+1} \alpha \int_{P_{+}} \frac{\operatorname{Arg}(u)}{u+2 \alpha} \frac{u_{1}^{r} u_{2}^{n-p}}{u^{n+r-p+1}} \partial_{1,2}^{p, n-p}(D \phi)(u+\alpha) d u\right| \\
& \leqslant 2^{n+r-p-1} \pi \sup _{u \in \mathbb{C}}\left|\partial_{1,2}^{p, n-p}(D \phi)(u)\right| \int_{\mathcal{K}-\alpha}\left|\frac{1}{u+2 \alpha}-\frac{1}{u}\right| d u .
\end{aligned}
$$

Comme

$$
\lim _{\alpha \rightarrow 0} \int_{\mathcal{K}}\left|\frac{1}{u+\alpha}-\frac{1}{u-\alpha}\right| d u=0
$$


d'après le théorème de convergence dominée, l'expression (3.3.6) devient, en considérant la relation (3.3.7) :

(3.3.8) $\sum_{p=0}^{n} \sum_{r=0}^{p} \sum_{k=0}^{n+r-p}(-1)^{k} \frac{2^{k} \mathcal{C}_{n}^{p} \mathcal{C}_{p}^{r}}{\alpha^{n+r-p-k}} \int_{P_{+}} \frac{\operatorname{Arg}(u)}{u^{k+1}} u_{1}^{r} u_{2}^{n-p} \partial_{1,2}^{p, n-p}(D \phi)(u+\alpha) d u+o(1)$

où $o(1)$ représente une fonction qui tend vers 0 si $\alpha$ tend vers 0 uniformément en $\phi$. On pose $\sigma=r+n-p$. L'expression (3.3.8) devient :

$$
\sum_{\sigma=0}^{n} \sum_{r=0}^{\sigma} \sum_{k=0}^{\sigma}(-1)^{k} \frac{2^{k} \mathcal{C}_{n}^{r+n-\sigma} \mathcal{C}_{r+n-\sigma}^{r}}{\alpha^{\sigma-k}} \int_{P_{+}} \frac{\operatorname{Arg}(u)}{u^{k+1}} u_{1}^{r} u_{2}^{\sigma-r} \partial_{1,2}^{r+n-\sigma, \sigma-r}(D \phi)(u+\alpha) d u .
$$

On remarque que l'on a l'égalité suivante :

$$
\mathcal{C}_{n}^{r+n-\sigma} \mathcal{C}_{r+n-\sigma}^{r}=\mathcal{C}_{n}^{\sigma} \mathcal{C}_{\sigma}^{r},
$$

On en déduit la nouvelle expression

$$
\sum_{\sigma=0}^{n} \sum_{r=0}^{\sigma} \sum_{k=0}^{\sigma}(-1)^{k} \frac{2^{k} \mathcal{C}_{n}^{\sigma} \mathcal{C}_{\sigma}^{r}}{\alpha^{\sigma-k}} \int_{P_{+}} \frac{\operatorname{Arg}(u)}{u^{k+1}} u_{1}^{r} u_{2}^{\sigma-r} \partial_{1,2}^{r, \sigma-r} \partial_{1}^{n-\sigma}(D \phi)(u+\alpha) d u .
$$

On considère les coordonnées polaires définies par $u=\rho e^{\mathrm{i} \theta} ;$ l'expression précédente devient :

$$
\begin{aligned}
& \sum_{\sigma=0}^{n} \sum_{k=0}^{\sigma} \sum_{r=0}^{\sigma} \\
& (-1)^{k} \frac{2^{k} \mathcal{C}_{n}^{\sigma} \mathcal{C}_{\sigma}^{r}}{\alpha^{\sigma-k}} \int_{\left.\mathbb{R}_{+} \times\right] 0, \pi[}^{\sigma-k} e^{-\mathrm{i}(k+1) \theta} \cos ^{r}(\theta) \sin ^{\sigma-r}(\theta) \partial_{1,2}^{r, \sigma-r} \partial_{1}^{n-\sigma}(D \phi)\left(\rho e^{\mathrm{i} \theta}+\alpha\right) d \rho d \theta .
\end{aligned}
$$

L'égalité suivante :

$$
\partial_{\rho}^{\sigma}\left(\partial_{1}^{n-\sigma}(D \phi)\left(\rho e^{\mathrm{i} \theta}+\alpha\right)\right)=\sum_{r=0}^{\sigma} \mathcal{C}_{\sigma}^{r} \cos ^{r}(\theta) \sin ^{\sigma-r}(\theta) \partial_{1,2}^{r, \sigma-r} \partial_{1}^{n-\sigma}(D \phi)\left(\rho e^{\mathrm{i} \theta}+\alpha\right)
$$

permet de réécrire (3.3.9) ainsi

$$
\sum_{\sigma=0}^{n} \sum_{k=0}^{\sigma}(-1)^{k} \frac{2^{k} \mathcal{C}_{n}^{\sigma}}{\alpha^{\sigma-k}} \int_{\left.\mathbb{R}_{+} \times\right] 0, \pi[} \theta \rho^{\sigma-k} e^{-\mathrm{i}(k+1) \theta} \partial_{\rho}^{\sigma}\left(\partial_{1}^{n-\sigma}(D \phi)\left(\rho e^{\mathrm{i} \theta}+\alpha\right)\right) d \rho d \theta .
$$

En intégrant en $\rho, \sigma-k$ fois, on obtient la nouvelle formule :

$$
\sum_{\sigma=0}^{n} \sum_{k=0}^{\sigma}(-1)^{\sigma} \frac{2^{k} \mathcal{C}_{n}^{\sigma}(\sigma-k) !}{\alpha^{\sigma-k}} \int_{\left.\mathbb{R}_{+} \times\right] 0, \pi[} \theta e^{-\mathrm{i}(k+1) \theta} \partial_{\rho}^{k}\left(\partial_{1}^{n-\sigma}(D \phi)\left(\rho e^{\mathrm{i} \theta}+\alpha\right)\right) d \rho d \theta .
$$

En revenant aux coordonnées $\left(u_{1}, u_{2}\right)$, cette expression devient

$$
\sum_{\sigma=0}^{n} \sum_{k=0}^{\sigma} \sum_{s=0}^{k}(-1)^{\sigma} \frac{2^{k} \mathcal{C}_{n}^{\sigma} \mathcal{C}_{k}^{s}(\sigma-k) !}{\alpha^{\sigma-k}} \int_{P_{+}} \frac{\operatorname{Arg}(u)}{u^{k+1}} u_{1}^{s} u_{2}^{k-s} \partial_{1,2}^{s, k-s} \partial_{1}^{n-\sigma}(D \phi)(u+\alpha) d u
$$


En utilisant le développement de Taylor de $\partial_{1,2}^{s, k-s} \partial_{1}^{n-\sigma}(D \phi)(u+\alpha)$ en $\alpha=0$ à l'ordre $\sigma-k$, on a :

$$
\begin{aligned}
& R_{1}+\sum_{\sigma=0}^{n} \sum_{k=0}^{\sigma} \sum_{s=0}^{k} \sum_{t=0}^{\sigma-k} \\
& \quad(-1)^{\sigma} \frac{2^{k} \mathcal{C}_{n}^{\sigma} \mathcal{C}_{k}^{s}(\sigma-k) !}{t ! \alpha^{\sigma-k-t}} \int_{P_{+}} \frac{\operatorname{Arg}(u)}{u^{k+1}} u_{1}^{s} u_{2}^{k-s} \partial_{1,2}^{s+t, k-s} \partial_{1}^{n-\sigma}(D \phi)(u) d u .
\end{aligned}
$$

où

$$
\begin{aligned}
R_{1}=\sum_{\sigma=0}^{n} \sum_{k=0}^{\sigma} \sum_{s=0}^{k}(-1)^{\sigma} \frac{2^{k} \mathcal{C}_{n}^{\sigma} \mathcal{C}_{k}^{s}(\sigma-k) !}{(\sigma-k) !} \\
\quad \int_{P_{+}} \frac{\operatorname{Arg}(u)}{u^{k+1}} u_{1}^{s} u_{2}^{k-s} \int_{0}^{\alpha}(\alpha-v)^{\sigma-k} \partial_{1,2}^{s+\sigma-k, k-s} \partial_{1}^{n-\sigma}(D \phi)(u+v) d v d u
\end{aligned}
$$

Montrons que $R_{1}$ tend vers 0 si $\alpha$ tend vers 0 uniformément en $\phi$. Soit $\mathcal{K}$ une partie compacte de $\mathbb{C}$ et $\phi \in \mathcal{C}_{\mathcal{K}}^{n+1}(\mathbb{C})$. On pose $\mathcal{K}_{1}=\mathcal{K}+[-1,1]$, on a alors pour $|\alpha| \leqslant 1$,

$$
\begin{aligned}
& \left|\int_{P_{+}} \frac{\operatorname{Arg}(u)}{u^{k+1}} u_{1}^{s} u_{2}^{k-s} \int_{0}^{\alpha}(\alpha-v)^{\sigma-k} \partial_{1,2}^{s+\sigma-k, k-s} \partial_{1}^{n-\sigma}(D \phi)(u+v) d v d u\right| \\
& \leqslant \int_{\mathcal{K}_{1}}\left|\frac{\operatorname{Arg}(u)}{u^{k+1}} u_{1}^{s} u_{2}^{k-s}\right| d u\left|\int_{0}^{\alpha}\right|(\alpha-v)^{\sigma-k}|d v| \sup _{x \in \mathbb{C}}\left|\partial_{1,2}^{s+\sigma-k, k-s} \partial_{1}^{n-\sigma}(D \phi)(x)\right| \\
& \leqslant \frac{|\alpha|^{\sigma-k+1}}{\sigma-k+1} \int_{\mathcal{K}_{1}}\left|\frac{\operatorname{Arg}(u)}{u^{k+1}} u_{1}^{s} u_{2}^{k-s}\right| d u \sup _{x \in \mathbb{C}}\left|\partial_{1,2}^{s+\sigma-k, k-s} \partial_{1}^{n-\sigma}(D \phi)(x)\right|
\end{aligned}
$$

On en déduit la propriété annoncée, on peut donc négliger le terme $R_{1}$ et ne considérer que l'expression

$$
\sum_{\sigma=0}^{n} \sum_{k=0}^{\sigma} \sum_{s=0}^{k} \sum_{t=0}^{\sigma-k}(-1)^{\sigma} \frac{2^{k} \mathcal{C}_{n}^{\sigma} \mathcal{C}_{k}^{s}(\sigma-k) !}{t ! \alpha^{\sigma-k-t}} \int_{P_{+}} \frac{\operatorname{Arg}(u)}{u^{k+1}} u_{1}^{s} u_{2}^{k-s} \partial_{1,2}^{s+t, k-s} \partial_{1}^{n-\sigma}(D \phi)(u) d u
$$

qui s'exprime en posant $u=\rho e^{\mathrm{i} \theta}$,

$$
\sum_{\sigma=0}^{n} \sum_{t=0}^{\sigma} \sum_{k=0}^{\sigma-t}(-1)^{\sigma} \frac{2^{k} \mathcal{C}_{n}^{\sigma}(\sigma-k) !}{t ! \alpha^{\sigma-t-k}} \int_{\left.\mathbb{R}_{+} \times\right] 0, \pi[} \theta e^{-\mathrm{i}(k+1) \theta} \partial_{\rho}^{k}\left(\partial_{1}^{n-\sigma+t}(D \phi)\left(\rho e^{\mathrm{i} \theta}\right)\right) d \rho d \theta .
$$

Ensuite, en posant $\nu=\sigma-t-k$, l'expression précédente devient

$$
\begin{aligned}
\sum_{\nu=0}^{n} \frac{1}{\alpha^{\nu}}\left\{\sum_{\sigma=\nu}^{n} \sum_{t=0}^{\sigma-\nu}(-1)^{\sigma} \frac{2^{\sigma-t-\nu} \mathcal{C}_{n}^{\sigma}(t+\nu) !}{t !}\right. \\
\left.\int_{\left.\mathbb{R}_{+} \times\right] 0, \pi[} \theta e^{-\mathrm{i}(\sigma-t-\nu+1) \theta} \partial_{\rho}^{\sigma-t-\nu}\left(\partial_{1}^{n-\sigma+t}(D \phi)\left(\rho e^{\mathrm{i} \theta}\right)\right) d \rho d \theta\right\} .
\end{aligned}
$$


En considérant le changement d'indice $\sigma \rightarrow \sigma+\nu$, l'expression ci-dessus s'écrit :

$$
\sum_{\nu=0}^{n} \frac{(-1)^{\nu}}{\alpha^{\nu}} \Gamma_{\nu}
$$

où

$$
\begin{aligned}
\Gamma_{\nu}= & \sum_{\sigma=0}^{n-\nu} \sum_{t=0}^{\sigma} \\
& \quad(-1)^{\sigma} \frac{2^{\sigma-t} \mathcal{C}_{n}^{\sigma+\nu}(t+\nu) !}{t !} \int_{\left.\mathbb{R}_{+} \times\right] 0, \pi[} \theta e^{-\mathrm{i}(\sigma-t+1) \theta} \partial_{\rho}^{\sigma-t}\left(\partial_{1}^{n-\sigma-\nu+t}(D \phi)\left(\rho e^{\mathrm{i} \theta}\right)\right) d \rho d \theta .
\end{aligned}
$$

L'étape suivante est d'exprimer $\Gamma_{\nu}$ en une somme de $\partial_{1,2}^{p, q} \phi(0)$. En posant $\eta=\sigma-t$ puis $\sigma \rightarrow \sigma+\eta$, on obtient :

$$
\begin{aligned}
\Gamma_{\nu} & =\sum_{\eta=0}^{n-\nu} \sum_{\sigma=\eta}^{n-\nu} \\
& \frac{(-1)^{\sigma} 2^{\eta} \mathcal{C}_{n}^{\sigma+\nu}(\sigma-\eta+\nu) !}{(\sigma-\eta) !} \int_{\left.\mathbb{R}_{+} \times\right] 0, \pi[} \theta e^{-\mathrm{i}(\eta+1) \theta} \partial_{\rho}^{\eta}\left(\partial_{1}^{n-\eta-\nu}(D \phi)\left(\rho e^{\mathrm{i} \theta}\right)\right) d \rho d \theta \\
= & \sum_{\eta=0}^{n-\nu}(-1)^{\eta} 2^{\eta} \sum_{\sigma=0}^{n-\nu-\eta} \\
& \frac{(-1)^{\sigma} \mathcal{C}_{n}^{\sigma+\eta+\nu}(\sigma+\nu) !}{\sigma !} \int_{\left.\mathbb{R}_{+} \times\right] 0, \pi[} \theta e^{-\mathrm{i}(\eta+1) \theta} \partial_{\rho}^{\eta}\left(\partial_{1}^{n-\nu-\eta}(D \phi)\left(\rho e^{\mathrm{i} \theta}\right)\right) d \rho d \theta
\end{aligned}
$$

Le terme d'indice $\eta=0$ de la somme précédente se calcule ainsi :

$$
\begin{aligned}
& \sum_{\sigma=0}^{n-\nu} \frac{(-1)^{\sigma} n !}{(n-\sigma-\nu) ! \sigma !} \int_{\left.\mathbb{R}_{+} \times\right] 0, \pi[} \theta e^{-\mathrm{i} \theta} \partial_{1}^{n-\nu}(D \phi)\left(\rho e^{i \theta}\right) d \rho d \theta \\
& =\frac{n !}{(n-\nu) !}\left(\sum_{\sigma=0}^{n-\nu}(-1)^{\sigma} \mathcal{C}_{n-\nu}^{\sigma}\right) \int_{\left.\mathbb{R}_{+} \times\right] 0, \pi[} \theta e^{-\mathrm{i} \theta} \partial_{1}^{n-\nu}(D \phi)\left(\rho e^{\mathrm{i} \theta}\right) d \rho d \theta .
\end{aligned}
$$

Si $\nu<n$, cette expression est nulle. Les autres termes d'indice $\eta \geqslant 1$ s'écrivent :

$$
\begin{aligned}
& -\sum_{\eta=1}^{n-\nu}(-1)^{\eta} 2^{\eta} \sum_{\sigma=0}^{n-\nu-\eta} \\
& \quad \frac{(-1)^{\sigma} \mathcal{C}_{n}^{\sigma+\eta+\nu}(\sigma+\nu) !}{\sigma !} \int_{0}^{\pi} \theta e^{-\mathrm{i}(\eta+1) \theta} \partial_{\rho}^{\eta-1}\left(\partial_{1}^{n-\nu-\eta}(D \phi)\left(\rho e^{\mathrm{i} \theta}\right)\right)_{\rho=0} d \theta .
\end{aligned}
$$

L'intégrale ci-dessus s'écrit ainsi :

$$
\begin{aligned}
& \int_{0}^{\pi} \theta e^{-\mathrm{i}(\eta+1) \theta} \partial_{\rho}^{\eta-1}\left(\partial_{1}^{n-\nu-\eta}(D \phi)\left(\rho e^{\mathrm{i} \theta}\right)\right)_{\rho=0} d \theta \\
= & \sum_{b=0}^{\eta-1} \mathcal{C}_{\eta-1}^{b} \int_{0}^{\pi} \theta e^{-\mathrm{i}(\eta+1) \theta} \cos ^{b}(\theta) \sin ^{\eta-1-b}(\theta) d \theta \partial_{1,2}^{n+b-\nu-\eta+b, \eta-1-b}(D \phi)(0) .
\end{aligned}
$$


Ainsi, pour $\nu<n$, on en déduit qu'il existe une famille de nombres complexes $\left(\Gamma_{\nu, s}\right)_{s=0, \ldots, n-\nu-1}$ telle que

$$
\Gamma_{\nu}=\sum_{s=0}^{n-\nu-1} \Gamma_{\nu, s} \partial_{1,2}^{s, n-s-\nu-1}(D \phi)(0)
$$

D'après l'expression (3.3.12), on sait que la fonction

$$
\alpha \in \mathbb{R}^{*} \longmapsto|\alpha| \Omega_{\phi}^{(n)}(\alpha)
$$

possède un développement asymptotique en 0 en puissances de $\alpha^{-\nu}$ avec $0 \leqslant \nu \leqslant n$. Ainsi la partie impaire de la partie réelle de cette fonction

$$
\alpha \longmapsto \Re\left(|\alpha| \Omega_{\phi}^{(n)}(\alpha)\right)-\Re\left(|\alpha| \Omega_{\phi}^{(n)}(-\alpha)\right)
$$

possède un développement asymptotique en puissances de $\alpha^{\nu}$ avec $\nu$ impair et $0 \leqslant \nu<n$. D'après (3.3.15), les coefficients de ce développement dépendent des dérivées d'ordre $n-\nu$ qui sont nulles d'après (3.3.4) puisque $n$ est pair et $\nu$ impair. Ainsi, cette fonction se prolonge par continuité en 0 et tend vers 0 si $\alpha$ tend vers 0 uniformément en $\phi$.

On s'intéresse maintenant au calcul du développement asymptotique de $\varepsilon \Omega_{\phi}^{(n-1)}(\alpha)$ en 0 ( $n$ désigne toujours un entier pair). On rappelle que $\varepsilon=\operatorname{sign}(\alpha)$. On a, en considérant l'égalité (3.3.5) :

$$
\varepsilon \Omega_{\phi}^{(n-1)}(\alpha)=\sum_{p=0}^{n-1} \sum_{r=0}^{p} \frac{\mathcal{C}_{n-1}^{p} \mathcal{C}_{p}^{r}}{\alpha^{n+r-p}} \int_{P_{+}} \frac{\operatorname{Arg}(u)}{u+2 \alpha} u_{1}^{r} u_{2}^{n-p-1} \partial_{1,2}^{p, n-1-p}(D \phi)(u+\alpha) d u
$$

On va considérer la partie impaire (en $\alpha$ ) de l'expression (3.3.16). On fixe $p, r$ et $n$. Il existe deux fonctions $\Gamma_{1}$ et $\Gamma_{2}$ sur $\mathbb{C} \times \mathbb{R}$ paires en la deuxième variable telles que

$$
\partial_{1,2}^{p, n-1-p}(D \phi)(u+\alpha)=\Gamma_{1}(u, \alpha)+\alpha \Gamma_{2}(u, \alpha)
$$

pour $u \in \mathbb{C}$ et $\alpha \in \mathbb{R}$. On souhaite tout d'abord étudier la partie impaire de l'expression

$$
\frac{1}{\alpha^{n+r-p}} \int_{P_{+}} \frac{\operatorname{Arg}(u)}{u+2 \alpha} u_{1}^{r} u_{2}^{n-p-1} \Gamma_{1}(u, \alpha) d u .
$$

On a la relation si $u+2 \alpha \neq 0$

$$
\frac{1}{u+2 \alpha}=\sum_{k=0}^{n+r-p}(-1)^{k} \frac{2^{k} \alpha^{k}}{u^{k+1}}+\left(-\frac{2 \alpha}{u}\right)^{n+r+1-p} \frac{1}{u+2 \alpha} .
$$

Le développement (3.3.18) multiplié par $1 / \alpha^{n+r-p}$ est égal à

$$
\frac{1}{\alpha^{n+r-p}(u+2 \alpha)}=\sum_{k=0}^{n+r-p}(-1)^{k} \frac{2^{k} \alpha^{k+p-n-r}}{u^{k+1}}+\left(-\frac{2}{u}\right)^{n+r-p}\left(\frac{1}{u+2 \alpha}-\frac{1}{u}\right) .
$$


La partie impaire de cette expression s'écrit

$$
\begin{aligned}
\text { 19) } & \frac{1}{2}\left\{\frac{1}{\alpha^{n+r-p}(u+2 \alpha)}-\frac{1}{(-\alpha)^{n+r-p}(u-2 \alpha)}\right\} \\
= & \sum_{\substack{k \in\{0, \ldots, n+r-p\} \\
k+p-n-r \text { impair }}}(-1)^{k} \frac{2^{k} \alpha^{k+p-n-r}}{u^{k+1}}+\frac{1}{2}\left(-\frac{2}{u}\right)^{n+r-p+1}\left(\frac{1}{u+2 \alpha}-\frac{1}{u-2 \alpha}\right) .
\end{aligned}
$$

On observe alors que chacun des termes de l'expression précédente multipliée par $u_{1}^{r} u_{2}^{n-1-p}$ est une fonction localement intégrable en $u$. En utilisant l'égalité (3.3.19) dans l'expression (3.3.17), on obtient

$$
\sum_{k=0}^{n+r-p}(-1)^{k} \frac{2^{k} \mathcal{C}_{n-1}^{p} \mathcal{C}_{p}^{r}}{t ! \alpha^{n+r-p-k}} \int_{P_{+}} \frac{\operatorname{Arg}(u)}{u^{k+1}} u_{1}^{r} u_{2}^{n-1-p} \Gamma_{1}(u, \alpha) d u+R_{1}
$$

avec $k+p-n-r$ impair et $R_{1}$ est égal à l'intégrale

$$
(-2)^{n+r-p} \int_{P_{+}}\left(\frac{1}{u+2 \alpha}-\frac{1}{u-2 \alpha}\right) \frac{\operatorname{Arg}(u)}{u^{n+r-p}} u_{1}^{r} u_{2}^{n-1-p} \Gamma_{1}(u, \alpha) d u
$$

On montre que cette expression tend vers 0 si $\alpha$ tend vers 0 uniformément en $\phi$. Soit $\mathcal{K}$ une partie compacte de $\mathbb{C}$ et $\phi \in \mathcal{C}_{\mathcal{K}}^{n+1}(\mathbb{C})$, on a l'inégalité

$$
\begin{array}{r}
\left|(-2)^{n+r-p} \int_{P_{+}}\left(\frac{1}{u+2 \alpha}-\frac{1}{u-2 \alpha}\right) \frac{\operatorname{Arg}(u)}{u^{n+r-p}} u_{1}^{r} u_{2}^{n-1-p} \Gamma_{1}(u, \alpha) d u\right| \\
\leqslant 2^{n+r-p-1} \pi \sup _{u \in \mathcal{K}-\alpha}\left|\Gamma_{1}(u, \alpha)\right| \int_{\mathcal{K}-\alpha}\left|\frac{1}{u+2 \alpha}-\frac{1}{u-2 \alpha}\right| d u
\end{array}
$$

Comme

$$
\lim _{\alpha \rightarrow 0} \int_{\mathcal{K}-\alpha}\left|\frac{1}{u+2 \alpha}-\frac{1}{u-2 \alpha}\right| d u=0
$$

la propriété est démontrée et $R_{1}$ tend vers 0 si $\alpha$ tend vers 0 uniformément en $\phi$. Ensuite, il s'agit de procéder de la même manière avec la fonction $\Gamma_{2}$. On en déduit que la partie impaire de l'expression (3.3.16) possède le même développement asymptotique en 0 que la partie impaire de

$$
\sum_{p=0}^{n-1} \sum_{r=0}^{p} \sum_{k=0}^{n+r-p}(-1)^{k} \frac{2^{k} \mathcal{C}_{n-1}^{p} \mathcal{C}_{p}^{r}}{\alpha^{n+r-p-k}} \int_{P_{+}} \frac{\operatorname{Arg}(u)}{u^{k+1}} u_{1}^{r} u_{2}^{n-1-p} \partial_{1,2}^{p, n-1-p}(D \phi)(u+\alpha) d u
$$


En posant $\sigma=n+r-p-1$ puis $\sigma \rightarrow n-1-\sigma$ l'expression précédente devient :

$\sum_{p=0}^{n-1} \sum_{\sigma=n-p-1}^{n-1} \sum_{k=0}^{\sigma+1}$

$(-1)^{k} \frac{2^{k} \mathcal{C}_{n-1}^{p} \mathcal{C}_{p}^{\sigma+p+1-n}}{\alpha^{\sigma+1-k}} \int_{P_{+}} \frac{\operatorname{Arg}(u)}{u^{k+1}} u_{1}^{\sigma+p+1-n} u_{2}^{n-1-p} \partial_{1,2}^{p, n-1-p}(D \phi)(u+\alpha) d u$

$\sum_{\sigma=0}^{n-1} \sum_{p=\sigma}^{n-1} \sum_{k=0}^{n-\sigma}$

$(-1)^{k} \frac{2^{k} \mathcal{C}_{n-1}^{p} \mathcal{C}_{p}^{p-\sigma}}{\alpha^{n-\sigma-k}} \int_{P_{+}} \frac{\operatorname{Arg}(u)}{u^{k+1}} u_{1}^{p-\sigma} u_{2}^{n-1-p} \partial_{1,2}^{p, n-1-p}(D \phi)(u+\alpha) d u$,

puis en posant $p \rightarrow p+\sigma$, on obtient :

$\sum_{\sigma=0}^{n-1} \sum_{p=0}^{n-1-\sigma} \sum_{k=0}^{n-\sigma}$

$(-1)^{k} \frac{2^{k} \mathcal{C}_{n-1}^{p+\sigma} \mathcal{C}_{p+\sigma}^{p}}{\alpha^{n-\sigma-k}} \int_{P_{+}} \frac{\operatorname{Arg}(u)}{u^{k+1}} u_{1}^{p} u_{2}^{n-1-p-\sigma} \partial_{1,2}^{p, n-1-p-\sigma} \partial_{1}^{\sigma}(D \phi)(u+\alpha) d u$.

En considérant les coordonnées polaires $u=\rho e^{\mathrm{i} \theta}$ puis en intégrant par parties, on obtient :

$$
\begin{aligned}
& \sum_{\sigma=0}^{n-1} \sum_{k=0}^{n-\sigma} \sum_{p=0}^{n-1-\sigma}(-1)^{k} \frac{2^{k} \mathcal{C}_{n-1}^{\sigma} \mathcal{C}_{n-1-\sigma}^{p}}{\alpha^{n-\sigma-k}} \\
& \int_{\left.\mathbb{R}_{+} \times\right] 0, \pi[}^{\theta \rho^{n-1-\sigma-k}} e^{-\mathrm{i}(k+1) \theta} \cos ^{p}(\theta) \sin ^{n-1-p-\sigma}(\theta) \partial_{1,2}^{p, n-1-p-\sigma} \partial_{1}^{\sigma}(D \phi)\left(\rho e^{\mathrm{i} \theta}+\alpha\right) d \rho d \theta \\
& =\sum_{\sigma=0}^{n-1} \sum_{k=0}^{n-\sigma} \\
& (-1)^{k} \frac{2^{k} \mathcal{C}_{n-1}^{\sigma}}{\alpha^{n-\sigma-k}} \int_{\left.\mathbb{R}_{+} \times\right] 0, \pi[} \theta \rho^{n-1-\sigma-k} e^{-\mathrm{i}(k+1) \theta} \partial_{\rho}^{n-1-\sigma}\left(\partial_{1}^{\sigma}(D \phi)\left(\rho e^{\mathrm{i} \theta}+\alpha\right)\right) d \rho d \theta .
\end{aligned}
$$

En effectuant $n-1-\sigma-k$ intégrations par parties dans chacune des intégrales cidessus, on en déduit l'expression suivante :

$$
\begin{aligned}
& \sum_{\sigma=0}^{n-1} \sum_{k=0}^{n-\sigma} \\
& (-1)^{n-1-\sigma} \frac{2^{k} \mathcal{C}_{n-1}^{\sigma}(n-1-\sigma-k) !}{\alpha^{n-\sigma-k}} \int_{\left.\mathbb{R}_{+} \times\right] 0, \pi[} e^{-\mathrm{i}(k+1) \theta} \partial_{\rho}^{k}\left(\partial_{1}^{\sigma}(D \phi)\left(\rho e^{\mathrm{i} \theta}+\alpha\right)\right) d \rho d \theta .
\end{aligned}
$$


En revenant aux coordonnées cartésiennes $\left(u_{1}, u_{2}\right)$, on obtient :

$$
\begin{aligned}
& \sum_{\sigma=0}^{n-1} \sum_{k=0}^{n-\sigma} \sum_{r=0}^{k} \\
& (-1)^{n-1-\sigma} \frac{2^{k} \mathcal{C}_{n-1}^{\sigma} \mathcal{C}_{k}^{r}(n-1-\sigma-k) !}{\alpha^{n-\sigma-k}} \int_{P_{+} u^{k+1}}^{\operatorname{Arg}(u)} u_{1}^{r} u_{2}^{k-r} \partial_{1,2}^{r+\sigma-k-r}(D \phi)(u+\alpha) d u
\end{aligned}
$$

On peut à présent considérer le développement de Taylor de $\partial_{1,2}^{r+\sigma, k-r}(D \phi)(u+\alpha)$ en $\alpha=0$ à l'ordre $n-\sigma-k$, on obtient que le reste qui apparaît tend uniformément vers 0 quand $\alpha$ tend vers 0 . On est ramené à considérer la somme suivante :

$$
\begin{aligned}
& \sum_{\sigma=0}^{n-1} \sum_{k=0}^{n-\sigma} \sum_{r=0}^{k} \sum_{t=0}^{n-\sigma-k} \\
& \quad(-1)^{n-1-\sigma} \frac{2^{k} \mathcal{C}_{n-1}^{\sigma} \mathcal{C}_{k}^{r}(n-1-\sigma-k) !}{t ! \alpha^{n-\sigma-k-t}} \int_{P_{+}} \frac{\operatorname{Arg}(u)}{u^{k+1}} u_{1}^{r} u_{2}^{k-r} \partial_{1,2}^{r+\sigma+t, k-r}(D \phi)(u) d u .
\end{aligned}
$$

En coordonnées polaires cette expression s'écrit :

$$
\begin{aligned}
& \sum_{\sigma=0}^{n-1} \sum_{k=0}^{n-\sigma} \sum_{r=0}^{k} \sum_{t=0}^{n-\sigma-k} \\
& \quad(-1)^{n-1-\sigma} \frac{2^{k} \mathcal{C}_{n-1}^{\sigma} \mathcal{C}_{k}^{r}(n-1-\sigma-k) !}{t ! \alpha^{n-\sigma-k-t}} \int_{\left.\mathbb{R}_{+} \times\right] 0, \pi[} \theta \rho^{-i(k+1)} \partial_{\rho}^{k}\left(\partial_{1}^{\sigma}(D \phi)\left(\rho e^{\mathrm{i} \theta}\right)\right) d \rho d \theta .
\end{aligned}
$$

On remarque que les intégrales qui apparaissent dans cette expression sont identiques à celles de l'expression (3.3.13). On en déduit en posant $\nu=n-\sigma-k-t$ que la partie impaire (en $\alpha$ ) de l'expression précédente, s'écrit

$$
\sum_{\substack{\nu=0 \\ \nu \text { impair }}}^{n} \frac{(-1)^{\nu}}{\alpha^{\nu}} \Delta_{\nu}
$$

où

$$
\Delta_{\nu}=\sum_{s=0}^{n-\nu} \Delta_{\nu, s} \partial_{1,2}^{s, n-\nu-s} \phi(0)
$$

avec $\Delta_{\nu, s} \in \mathbb{C}$. Comme $n$ est pair et $\nu$ impair, $s$ et $n-\nu-s$ sont de parités différentes ainsi on a $\partial_{1,2}^{s, n-\nu-s} \phi(0)=0$ et la fonction

$$
\alpha \longmapsto \operatorname{sign}(\alpha) \Re\left(\Omega_{\phi}^{(n)}(\alpha)+\Omega_{\phi}^{(n)}(-\alpha)\right)
$$

se prolonge par continuité en 0 . De plus, les termes négligés au cours du calcul tendent vers 0 si $\alpha$ tend vers 0 uniformément en $\phi$. On en déduit que le lemme est démontré.

Démonstration de la proposition 3.3.2. - Il suffit de démontrer le résultat suivant :

Pour $\phi \in \mathcal{C}_{c}^{d+1}(\mathbb{C})$ à valeurs réelles avec $d$ pair, on a $\widehat{\Xi}_{\phi} \in \mathcal{C}^{d}\left(\mathbb{R}^{2}\right)$. 
On pose $x_{1}^{\prime}=\beta-\alpha$ et $x_{2}^{\prime}=\beta+\alpha$. On a alors l'égalité :

$$
\begin{aligned}
\Xi_{\phi}\left(x_{1}^{\prime}, x_{2}^{\prime}\right) & =\int_{\left.\mathbb{R}_{+} \times\right] 0, \pi[}\left(\cos (\theta) \partial_{1} \phi+\sin (\theta) \partial_{2} \phi\right)\left(\rho e^{\mathrm{i} \theta}+\beta-\alpha\right) \operatorname{Arg}\left(\rho e^{\mathrm{i} \theta}-2 \alpha\right) d \rho d \theta \\
& =\Xi_{\phi(\beta+.)}(-\alpha, \alpha) .
\end{aligned}
$$

En considérant le changement de variable $z=\rho e^{\mathrm{i} \theta}$ et en utilisant (3.3.1), on obtient

$$
\Re\left(\widetilde{\Xi}_{\phi(\beta+.)}(\alpha)\right)=\Xi_{\phi(\beta+.)}(-\alpha, \alpha) .
$$

D'après le lemme 3.3.3, on en déduit que la fonction

$$
(\alpha, \beta) \longmapsto \Xi_{\phi}(\beta-\alpha, \beta+\alpha)
$$

est de classe $C^{d}$ sur l'ouvert $\{\alpha \neq 0\} \subset \mathbb{R}^{2} ;$ de même pour $\widehat{\Xi}_{\phi}$. Pour $n \leqslant d$, on a

$$
\partial_{\beta}^{n}\left(\widehat{\Xi}_{\phi}(\beta-\alpha, \beta+\alpha)\right)=\widehat{\Xi}_{\partial_{1}^{n}(\phi)}(\beta-\alpha, \beta+\alpha),
$$

ainsi en montrant que pour toute fonction $\phi \in \mathcal{C}_{c}^{n+1}(\mathbb{C})$ et tout entier pair $n$ la fonction

$$
(\alpha, \beta) \longmapsto \partial_{\alpha}^{n}\left(\widehat{\Xi}_{\phi}(\beta-\alpha, \beta+\alpha)\right)
$$

se prolonge par continuité sur $\mathbb{R}^{2}$ on aura démontré le lemme. En effet, si ce prolongement existe, la fonction

$$
\partial_{\alpha}^{m}\left(\widehat{\Xi}_{\phi}(\beta-\alpha, \beta+\alpha)\right)
$$

pour $m$ impair tel que $m<n$ admet un prolongement par continuité sur les ensembles $\{\alpha \geqslant 0\}$ et $\{\alpha \leqslant 0\}$ qui coïncide puisque la fonction est paire en $\alpha$ ainsi cette fonction se prolonge sur $\mathbb{R}^{2}$.

Pour une fonction $\phi \in \mathcal{C}_{c}^{n+1}(\mathbb{C})$, on note $\phi_{i}$ (resp. $\phi_{p}$ ) sa partie impaire (resp. sa partie paire). On a ainsi

$$
\phi=\phi_{i}+\phi_{p}
$$

Soit $\phi \in \mathcal{C}_{c}^{n+1}(\mathbb{C})$. On a l'égalité

$$
\widehat{\Xi}_{\phi(\beta+.)}(\alpha)=\widehat{\Xi}_{\phi(\beta+.)_{i}}(\alpha)+\widehat{\Xi}_{\phi(\beta+.)_{p}}(\alpha) .
$$

D'après le lemme 3.3.4, on a

$$
\widehat{\Xi}_{\phi(\beta+.)_{i}}(\alpha)=\frac{\pi^{2}}{2}(\phi(\beta+\alpha)-\phi(\beta-\alpha)) ;
$$

cette fonction appartient à $\mathcal{C}_{c}^{n+1}\left(\mathbb{R}^{2}\right)$. Soit $n \in \mathbb{N}$ pair. Montrons maintenant que

$$
(\alpha, \beta) \longmapsto \partial_{\alpha}^{n}\left(\widehat{\Xi}_{\phi(\beta+.)_{p}}(-\alpha, \alpha)\right)
$$

se prolonge par continuité à $\mathbb{R}^{2}$. Soit $\mathcal{K}_{1}$ (resp. $\mathcal{K}_{2}$ ) une partie compacte symétrique ${ }^{(3)}$ de $\mathbb{C}($ resp. $\mathbb{R})$ telle que

$$
\operatorname{Supp}(\phi)+\mathcal{K}_{2} \subset \mathcal{K}_{1}
$$

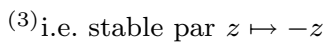


Soit $\varepsilon>0$, d'après le lemme 3.3.5, il existe $w_{1}, \ldots, w_{m} \in \operatorname{Sym}(\mathbb{C})$ de degrés au plus $n+1$ et $\eta>0$ tel que $|\alpha| \leqslant \eta$ entraîne

$$
\left|\partial_{\alpha}^{n}\left(\widehat{\Xi}_{\psi}(-\alpha, \alpha)\right)\right| \leqslant \eta \sup _{\substack{x \in \mathcal{K}_{1} \\ 1 \leqslant i \leqslant m}}\left|\partial\left(w_{i}\right) \psi(x)\right|
$$

pour tout $\psi \in \mathcal{C}_{\mathcal{K}_{1}}^{n+1}(\mathbb{C})$ vérifiant les hypothèses du lemme. On remarque ensuite que $\operatorname{pour} \beta \in \mathcal{K}_{2}$,

$$
\phi(\beta+.)_{p} \in \mathcal{C}_{\mathcal{K}_{1}}^{n+1}(\mathbb{C})
$$

et

$$
\sup _{\substack{x \in \mathcal{K}_{1} \\ 1 \leqslant i \leqslant m}}\left|\partial\left(w_{i}\right) \phi(\beta+.)_{p}\right| \leqslant \sup _{\substack{x \in \mathbb{C} \\ 1 \leqslant i \leqslant m}}\left|\partial\left(w_{i}\right) \phi(x)\right| .
$$

Ainsi, l'inégalité (3.3.21) permet d'obtenir

$$
\left|\partial_{\alpha}^{n}\left(\widehat{\Xi}_{\phi(\beta+.)_{p}}(-\alpha, \alpha)\right)\right| \leqslant \varepsilon \sup _{\substack{x \in \mathbb{C} \\ 1 \leqslant i \leqslant m}}\left|\partial\left(w_{i}\right) \phi(x)\right|
$$

pour $\beta \in \mathcal{K}_{2}$ et $|\alpha| \leqslant \eta$. Comme la partie compacte $\mathcal{K}_{2}$ peut être prise aussi grande que l'on veut, on en déduit que l'application

$$
(\alpha, \beta) \longmapsto \partial_{\alpha}^{n}\left(\widehat{\Xi}_{\phi(\beta+.)_{p}}(-\alpha, \alpha)\right)
$$

se prolonge par continuité sur $\mathbb{R}^{2}$. La proposition est démontrée.

3.3.2. Étude des intégrales liées à la partie compacte. — Pour calculer les termes dépendant de $\psi_{\varnothing}$, on a besoin de quelques lemmes préparatoires. L'objet de cette sous-section est de montrer les lemmes 3.3.7, 3.3.8 et 3.3.12. Ces résultats sont utilisés dans la section suivante.

DÉFInITION 3.3.6. - On considère pour une fonction $\phi \in \mathcal{C}_{c}^{1}(\mathbb{R}), \varepsilon, \eta \in\{ \pm 1\}$ et $\alpha, \beta, x_{1}^{\prime}, x_{2}^{\prime} \in \mathbb{R}$ tels que $x_{1}^{\prime} \neq x_{2}^{\prime}$ et $\alpha \neq 0$ les intégrales suivantes :

$$
\begin{aligned}
F_{\phi}^{t}\left(x_{1}^{\prime}, x_{2}^{\prime}, \varepsilon, \eta\right) & =\int_{\mathbb{R}} \frac{\ln \left(v+\mathrm{i} t \varepsilon-x_{1}^{\prime}\right)}{v+\mathrm{i} t \eta-x_{2}^{\prime}} \phi(v) d v \quad \text { avec } t>0, \\
H_{\phi}(\beta) & =\int_{\mathbb{R}} \ln |u| \phi(u+\beta) d u \\
A_{\phi}(\alpha, \beta) & =\int_{\mathbb{R}} \omega(u)(\phi(2 \alpha u+\alpha+\beta)+\phi(-2 \alpha u-\alpha+\beta)) d u \\
B_{\phi}(\alpha, \beta) & =\ln |2 \alpha|\left(H_{\phi}^{\prime}(\beta+\alpha)-H_{\phi}^{\prime}(\beta-\alpha)\right), \\
C_{\phi}(\alpha, \beta) & =\int_{\mathbb{R}} \ln |u-\alpha| \ln |u+\alpha| \phi(u+\beta) d u
\end{aligned}
$$

où

$$
\omega(u)=\frac{\ln |1+u|}{u} \text { pour } u \in \mathbb{C} \backslash\{ \pm 1,0\}
$$


On pose aussi :

$$
I_{n}=\int_{-1}^{0} \frac{(1+x)^{n}-1}{x} d x \text { et } J_{n}=\int_{-2}^{0} \frac{(1+x)^{n}-1}{x} d x
$$

$\mu$ désigne la fonction caractéristique de l'intervalle $]-\infty, 0[$.

Lemme 3.3.7. - Pour $\phi \in \mathcal{C}_{c}^{1}(\mathbb{R})$, la limite

$$
\lim _{\substack{t \rightarrow 0 \\ t>0}} F_{\phi}^{t}\left(x_{1}^{\prime}, x_{2}^{\prime}, \varepsilon, \eta\right)
$$

existe; de plus, cette limite que l'on note $F_{\phi}\left(x_{1}^{\prime}, x_{2}^{\prime}, \varepsilon, \eta\right)$ vérifie

$$
\begin{aligned}
& F_{\phi}\left(x_{1}^{\prime}, x_{2}^{\prime}, \varepsilon, \eta\right) \\
& =\left\{\begin{array}{rr}
\ln \left(x_{2}^{\prime}-x_{1}^{\prime}\right)\left[\mathrm{i} \varepsilon \pi \phi\left(x_{1}^{\prime}\right)-\mathrm{i} \eta \pi \phi\left(x_{2}^{\prime}\right)-H_{\phi}^{\prime}\left(x_{2}^{\prime}\right)\right] \\
-\mathrm{i} \varepsilon \pi \int_{v \leqslant x_{1}^{\prime}} \ln \left|x_{2}^{\prime}-v\right| \phi^{\prime}(v) d v+\int_{\mathbb{R}} \omega(v) \phi(2 \alpha v+\alpha+\beta) d v \\
\ln \left(x_{1}^{\prime}-x_{2}^{\prime}\right)\left[\mathrm{i} \varepsilon \pi \phi\left(x_{1}^{\prime}\right)-\mathrm{i} \eta \pi \phi\left(x_{2}^{\prime}\right)-H_{\phi}^{\prime}<x_{2}^{\prime},\right. \\
-\mathrm{i} \varepsilon \pi \int_{v \leqslant x_{1}^{\prime}} \ln \left|x_{2}^{\prime}-v\right| \phi_{2}^{\prime}(v) d v-\int_{\mathbb{R}} \omega(v) \phi(2 \alpha v+\alpha+\beta) d v+\pi^{2} \varepsilon \eta \phi\left(x_{2}^{\prime}\right)
\end{array}\right. \\
& \text { si } x_{2}^{\prime}<x_{1}^{\prime} .
\end{aligned}
$$

Démonstration. - Premier cas : Si $x_{1}^{\prime}<x_{2}^{\prime}, F_{\phi}^{t}\left(x_{1}^{\prime}, x_{2}^{\prime}, \varepsilon, \eta\right)$ s'écrit :

$$
\int_{\mathbb{R}} \frac{\ln \left(x_{2}^{\prime}-x_{1}^{\prime}\right)}{v+\mathrm{i} t \eta-x_{2}^{\prime}} \phi(v) d v+\int_{\mathbb{R}} \frac{\ln \left(1+\frac{v+\mathrm{i} t \varepsilon-x_{2}^{\prime}}{x_{2}^{\prime}-x_{1}^{\prime}}\right)}{v+\mathrm{i} t \eta-x_{2}^{\prime}} \phi(v) d v .
$$

Le premier terme de l'expression précédente s'intègre par partie ainsi

$$
\int_{\mathbb{R}} \frac{\ln \left(x_{2}^{\prime}-x_{1}^{\prime}\right)}{v+\mathrm{i} t \eta-x_{2}^{\prime}} \phi(v) d v=-\ln \left(x_{2}^{\prime}-x_{1}^{\prime}\right) \int_{\mathbb{R}} \ln \left(v+\mathrm{i} t \eta-x_{2}^{\prime}\right) \phi^{\prime}(v) d v .
$$

En prenant la limite en $t=0$ de cette expression, on obtient l'égalité

$$
\lim _{\substack{t \rightarrow 0 \\ t>0}} \int_{\mathbb{R}} \frac{\ln \left(x_{2}^{\prime}-x_{1}^{\prime}\right)}{v+\mathrm{i} t \eta-x_{2}^{\prime}} \phi(v) d v=-\ln \left(x_{2}^{\prime}-x_{1}^{\prime}\right) H_{\phi}^{\prime}\left(x_{2}^{\prime}\right)-\mathrm{i} \pi \eta \ln \left(x_{2}^{\prime}-x_{1}^{\prime}\right) \phi\left(x_{2}^{\prime}\right) .
$$

Le deuxième terme de (3.3.22) s'écrit :

$$
\frac{1}{x_{2}^{\prime}-x_{1}^{\prime}} \int_{\mathbb{R}} \frac{\ln \left(1+\frac{v+\mathrm{i} t \varepsilon-x_{2}^{\prime}}{x_{2}^{\prime}-x_{1}^{\prime}}\right)}{\frac{v+\mathrm{i} t \varepsilon-x_{2}^{\prime}}{x_{2}^{\prime}-x_{1}^{\prime}}} \frac{v+\mathrm{i} t \varepsilon-x_{2}^{\prime}}{v+\mathrm{i} t \eta-x_{2}^{\prime}} \phi(v) d v .
$$

Soit $\mathcal{K}$ une partie compacte de $\mathbb{R}$ telle que $\operatorname{Supp}(\phi) \subset \mathcal{K}$. Comme la fonction $u \mapsto$ $\frac{\ln (1+u)}{u}$ est localement bornée sur l'ensemble $\left\{u \in \mathbb{C} \mid 1+u \in \mathbb{C} \backslash \mathbb{R}_{-}\right\}$, il existe une 
constante $C_{1}>0$ telle que

$$
\left|\frac{\ln \left(1+\frac{v+\mathrm{i} t \varepsilon-x_{2}^{\prime}}{x_{2}^{\prime}-x_{1}^{\prime}}\right)}{\frac{v+\mathrm{i} t \varepsilon-x_{2}^{\prime}}{x_{2}^{\prime}-x_{1}^{\prime}}}\right|<C_{1}
$$

pour $v \in \mathcal{K}$ et $0 \leqslant t \leqslant 1$. Ensuite, on a les relations suivantes

$$
\frac{v+\mathrm{i} t \varepsilon-x_{2}^{\prime}}{v+\mathrm{i} t \eta-x_{2}^{\prime}}=1+\mathrm{i} t \frac{\varepsilon-\eta}{v+\mathrm{i} t \eta-x_{2}^{\prime}} \text { et }\left|\frac{t}{v+\mathrm{i} t \eta-x_{2}^{\prime}}\right| \leqslant \sqrt{\frac{t}{2|\eta|\left|v-x_{2}^{\prime}\right|}} .
$$

On en déduit la majoration

$$
\begin{aligned}
\frac{1}{x_{2}^{\prime}-x_{1}^{\prime}}\left|\frac{\ln \left(1+\frac{v+\mathrm{i} t \varepsilon-x_{2}^{\prime}}{x_{2}^{\prime}-x_{1}^{\prime}}\right)}{\frac{v+\mathrm{i} t \varepsilon-x_{2}^{\prime}}{x_{2}^{\prime}-x_{1}^{\prime}}} \frac{v+\mathrm{i} t \varepsilon-x_{2}^{\prime}}{v+\mathrm{i} t \eta-x_{2}^{\prime}} \phi(v)\right| & \leqslant \frac{C_{1}}{x_{2}^{\prime}-x_{1}^{\prime}}\left(1+\frac{1}{\sqrt{2\left|v-x_{2}^{\prime}\right|}}\right)|\phi(v)|
\end{aligned}
$$

pour $v \in \mathbb{R}$ et $0<t \leqslant 1$. Cette inégalité permet d'appliquer le théorème de la convergence dominée, ainsi l'expression (3.3.23) converge en $t=0$ vers

$$
\int_{\mathbb{R}}\left(\frac{1}{x_{2}^{\prime}-x_{1}^{\prime}} \omega\left(\frac{v-x_{2}^{\prime}}{x_{2}^{\prime}-x_{1}^{\prime}}\right)+\mathrm{i} \varepsilon \pi \frac{\mu\left(\frac{v-x_{1}^{\prime}}{x_{2}^{\prime}-x_{1}^{\prime}}\right)}{v-x_{2}^{\prime}}\right) \phi(v) d v
$$

Cela montre l'existence de la limite du deuxième membre de (3.3.22). En posant $u=\frac{v-x_{2}^{\prime}}{x_{2}^{\prime}-x_{1}^{\prime}}$, l'expression précédente s'écrit :

$$
\mathrm{i} \varepsilon \pi \phi\left(x_{1}^{\prime}\right) \ln \left(x_{2}^{\prime}-x_{1}^{\prime}\right)-\mathrm{i} \varepsilon \pi \int_{v \leqslant x_{1}^{\prime}} \ln \left(x_{2}^{\prime}-v\right) \phi^{\prime}(v) d v+\int_{\mathbb{R}} \omega(u) \phi(2 \alpha u+\beta+\alpha) d u
$$

Ainsi, $F_{\phi}\left(x_{1}^{\prime}, x_{2}^{\prime}, \varepsilon, \eta\right)$ s'écrit :

$$
\begin{aligned}
\ln \left(x_{2}^{\prime}-x_{1}^{\prime}\right)\left[\mathrm{i} \varepsilon \pi \phi\left(x_{1}^{\prime}\right)\right. & \left.-\mathrm{i} \pi \eta \phi\left(x_{2}^{\prime}\right)-H_{\phi}^{\prime}\left(x_{2}^{\prime}\right)\right] \\
& -\mathrm{i} \varepsilon \pi \int_{v \leqslant x_{1}^{\prime}} \ln \left(x_{2}^{\prime}-v\right) \phi^{\prime}(v) d v+\int_{\mathbb{R}} \omega(u) \phi(2 \alpha u+\alpha+\beta) d u .
\end{aligned}
$$

Deuxième cas : Si $x_{2}^{\prime}<x_{1}^{\prime}$, on a la relation :

$$
\ln \left(v+\mathrm{i} t \varepsilon-x_{1}^{\prime}\right)=\ln \left(x_{1}^{\prime}-x_{2}^{\prime}\right)+\ln \left(1-\frac{v+\mathrm{i} t \varepsilon-x_{2}^{\prime}}{x_{1}^{\prime}-x_{2}^{\prime}}\right)+\mathrm{i} \pi \varepsilon .
$$


En appliquant de nouveau le théorème de convergence dominée, on montre que $F_{\phi}^{t}\left(x_{1}^{\prime}, x_{2}^{\prime}, \varepsilon, \eta\right)$ converge en $t=0$ vers

$$
\begin{aligned}
& -\left[\ln \left(x_{1}^{\prime}-x_{2}^{\prime}\right)+\mathrm{i} \pi \varepsilon\right]\left[H_{\phi}^{\prime}\left(x_{2}^{\prime}\right)+\mathrm{i} \pi \eta \phi\left(x_{2}^{\prime}\right)\right] \\
& -\int_{\mathbb{R}}\left(\frac{1}{x_{1}^{\prime}-x_{2}^{\prime}} \omega\left(\frac{x_{2}^{\prime}-v}{x_{1}^{\prime}-x_{2}^{\prime}}\right)-\mathrm{i} \pi \varepsilon \frac{\mu\left(\frac{x_{1}^{\prime}-v}{x_{1}^{\prime}-x_{2}^{\prime}}\right)}{x_{2}^{\prime}-v}\right) \phi(v) d v .
\end{aligned}
$$

Ainsi, $F_{\phi}\left(x_{1}^{\prime}, x_{2}^{\prime}, \varepsilon, \eta\right)$ s'écrit

$$
\begin{aligned}
{\left[\ln \left(x_{1}^{\prime}-x_{2}^{\prime}\right)+\mathrm{i} \pi \varepsilon\right]\left[-\mathrm{i} \pi \eta \phi\left(x_{2}^{\prime}\right)-H_{\phi}^{\prime}\left(x_{2}^{\prime}\right)\right]+\mathrm{i} \pi \varepsilon \ln \left(x_{1}^{\prime}-x_{2}^{\prime}\right) \phi\left(x_{1}^{\prime}\right) } \\
+\mathrm{i} \varepsilon \pi \int_{x_{1}^{\prime}}^{\infty} \ln \left(v-x_{2}^{\prime}\right) \phi^{\prime}(v) d v-\int_{\mathbb{R}} \omega(u) \phi(2 \alpha u+\alpha+\beta) d u .
\end{aligned}
$$

Cette expression s'écrit aussi

$$
\begin{array}{r}
\ln \left(x_{1}^{\prime}-x_{2}^{\prime}\right)\left[\mathrm{i} \varepsilon \pi \phi\left(x_{1}^{\prime}\right)-\mathrm{i} \eta \pi \phi\left(x_{2}^{\prime}\right)-\right. \\
\left.H_{\phi}^{\prime}\left(x_{2}^{\prime}\right)\right]-\mathrm{i} \varepsilon \pi \int_{v \leqslant x_{1}^{\prime}} \ln \left|x_{2}^{\prime}-v\right| \phi^{\prime}(v) d v \\
-\int_{\mathbb{R}} \omega(v) \phi(2 \alpha v+\alpha+\beta) d v+\pi^{2} \varepsilon \eta \phi\left(x_{2}^{\prime}\right) .
\end{array}
$$

Lemme 3.3.8. - Soit $\phi \in \mathcal{C}_{c}^{d+2}(\mathbb{R})$. Il existe une fonction $\Gamma \in \mathcal{C}^{d}\left(\mathbb{R}^{2}\right)$ telle que :

$$
C_{\phi}(\alpha, \beta)=\Gamma(\alpha, \beta)+\pi^{2} \int_{u \leqslant-|\alpha|} \phi(u+\beta) d u
$$

et

$$
\Gamma(\alpha, \beta)=\Gamma(-\alpha, \beta) .
$$

On en déduit alors :

$$
\left\{\begin{array}{lr}
\partial_{\alpha}^{n} C_{\phi}(\alpha, \beta) \text { est une fonction continue sur } \mathbb{R}^{2} & \text { si } n \text { est pair. } \\
\partial_{\alpha}^{n} C_{\phi}(\alpha, \beta)=-\operatorname{sign}(\alpha) \pi^{2} \phi^{(n-1)}(\beta) \text { modulo une fonction } \text { continue sur } \mathbb{R}^{2} & \text { si } n \text { est impair }
\end{array}\right.
$$

pour $n \leqslant d$.

Démonstration. - Il suffit de montrer la première partie du lemme, en effet en dérivant la relation (3.3.24) en $\alpha$ à l'ordre $n$ avec $n \geqslant 1$, on obtient

$$
\partial_{\alpha}^{n} C_{\phi}(\alpha, \beta)=\partial_{\alpha}^{n} \Gamma(\alpha, \beta)+\pi^{2}(-\operatorname{sign}(\alpha))^{n} \phi^{(n-1)}(\beta-|\alpha|) ;
$$

ainsi, pour $\varepsilon= \pm 1$

$$
\lim _{\substack{\alpha \rightarrow 0 \\ \operatorname{sign}(\alpha)=\varepsilon}} C_{\phi}^{(n)}(\alpha)= \begin{cases}\partial_{\alpha}^{n} \Gamma(0, \beta)+\pi^{2} \phi^{(n-1)}(\beta) & \text { si } n \text { est pair, } \\ -\pi^{2} \varepsilon \phi^{(n-1)}(\beta) & \text { si } n \text { est impair. }\end{cases}
$$

Pour $z \in \mathbb{C}$ avec $\Im(z) \neq 0$ et $\alpha \in \mathbb{R}$, on pose :

$$
f_{\alpha}(z)=\ln (\alpha+z) \ln (z-\alpha) .
$$


La fonction $f_{\alpha}$ est holomorphe sur l'ouvert $\{z \in C \mid \Im(z)>0\}$. Soit $\mathcal{K}$ un intervalle compact de $\mathbb{R}$. Il existe une constante $C>0$ telle que

$$
\sqrt{\varepsilon}|\ln (\alpha+x+\mathrm{i} \varepsilon)| \leqslant C \text { et } \sqrt{\varepsilon}|\ln (\alpha-x+\mathrm{i} \varepsilon)| \leqslant C
$$

pour $x, \alpha \in \mathcal{K}$ et $0<\varepsilon<1$. Ainsi, on a

$$
\left|f_{\alpha}(x+\mathrm{i} \varepsilon)\right| \leqslant C \varepsilon^{-1}
$$

pour $x, \alpha \in \mathcal{K}$ et $0<\varepsilon<1$. Soit $N \in \mathbb{N}$ avec $1 \leqslant N \leqslant d+1$; d'après l'inégalité précédente, on a

$$
\left|f_{\alpha}(x+\mathrm{i} \varepsilon)\right| \leqslant C \varepsilon^{-N} \forall \alpha, x \in \mathcal{K} \text { et } 0<\varepsilon<1 .
$$

D'après le théorème 3.1 .11 de $[\mathbf{3}]$, la limite

$$
\lim _{\substack{\varepsilon \rightarrow 0 \\ \varepsilon>0}} \int_{\mathbb{R}} \phi(u+\beta) f_{\alpha}(u+\mathbf{i} \varepsilon) d u
$$

existe et cette limite que l'on note

$$
\int_{\mathbb{R}} \phi(u+\beta) f_{\alpha}\left(u+\mathrm{i} 0_{+}\right) d u
$$

vérifie $[\mathbf{3}$, p. 65] :

$$
\begin{aligned}
\int_{\mathbb{R}} \phi(u+\beta) f_{\alpha}\left(u+\mathrm{i} 0_{+}\right) d u & =\int_{\mathbb{R}} \phi_{N}\left(u+\beta+\mathrm{i} \varepsilon_{0}\right) f_{\alpha}\left(u+\mathrm{i} \varepsilon_{0}\right) d u \\
+ & \frac{\left(\mathrm{i} \varepsilon_{0}\right)^{N+1}}{N !} \int_{\mathbb{R}} \int_{0}^{1} \phi^{(N+1)}(u+\beta) f_{\alpha}\left(u+\mathrm{i} t \varepsilon_{0}\right) t^{N} d t d u
\end{aligned}
$$

où $\varepsilon_{0}$ est un réel positif fixé et $\phi_{N}$ désigne la fonction définie sur $\mathbb{C}$ par :

$$
\phi_{N}(x+\mathrm{i} y)=\sum_{j=0}^{N} \frac{(\mathrm{i} y)^{j}}{j !} \phi^{(j)}(x) .
$$

Montrons que

$$
\int_{\mathbb{R}} \phi(u+\beta) f_{\alpha}\left(u+\mathrm{i} 0_{+}\right) d u
$$

est une fonction de classe $C^{d}$ sur $\mathbb{R}^{2}$. La fonction $f_{\alpha}\left(u+\mathrm{i} \varepsilon_{0}\right)$ est lisse par rapport à $\alpha$ ainsi on en déduit que

$$
\int_{\mathbb{R}} \phi(u+\beta) f_{\alpha}\left(u+\mathrm{i} \varepsilon_{0}\right) d u
$$

est de classe $C^{d}$ sur $\mathbb{R}^{2}$ (la dérivation en $\beta$ se fait sous le signe somme). Ensuite, on a

$$
\begin{aligned}
\partial_{\alpha}^{N-1} & f_{\alpha}\left(u+i t \varepsilon_{0}\right) t^{N} \\
& =\sum_{p=0}^{N-1}(-1)^{N-1-p} \mathcal{C}_{N-1}^{p} \ln ^{(p)}\left(\alpha+u+i t \varepsilon_{0}\right) \ln ^{(N-1-p)}\left(u-\alpha+i t \varepsilon_{0}\right) t^{N}
\end{aligned}
$$


Cette expression est bornée sur l'ensemble $\{(\alpha, u, t) \in \mathcal{K} \times \mathcal{K} \times] 0,1]\}$ en effet, pour $p \neq 0$, on a

$$
\left|\ln ^{(p)}\left(\alpha+u+i t \varepsilon_{0}\right)\right| \leqslant \frac{(p-1) !}{\varepsilon_{0}^{p} t^{p}}
$$

ainsi en utilisant les inégalités (3.3.25), on en déduit que l'expression (3.3.28) est bornée sur l'ensemble $\{(\alpha, u, t) \in \mathcal{K} \times \mathcal{K} \times] 0,1]\}$. D'après le théorème de dérivation sous le signe somme, on obtient que

$$
\int_{\mathbb{R}} \int_{0}^{1} \phi^{(N+1)}(u+\beta) f_{\alpha}\left(u+\mathrm{i} t \varepsilon_{0}\right) t^{N} d t d u
$$

est dérivable $N-1$ fois sur l'intérieur de $\mathcal{K}$; cela montre que

$$
\int_{\mathbb{R}} \phi(u+\beta) f_{\alpha}\left(u+\mathrm{i} 0_{+}\right) d u
$$

est dérivable $N-1$ fois en $\alpha$ sur l'intérieur de $\mathcal{K}$. Comme ce résultat est vrai pour $N \leqslant d+1$ et toute partie compacte assez grande, on obtient que cette fonction est de classe $C^{d}$ sur $\mathbb{R}^{2}$. Ensuite, on observe que l'on a l'égalité

$$
\begin{aligned}
\lim _{\substack{\varepsilon \rightarrow 0 \\
\varepsilon>0}} f_{\alpha}(u+\mathrm{i} \varepsilon)=\ln |\alpha+u| \ln |u-\alpha| & +\mathrm{i} \pi \mu(u-\alpha) \ln |\alpha+u| \\
& +\mathrm{i} \pi \mu(u+\alpha) \ln |u-\alpha|-\pi^{2} \mu(u-\alpha) \mu(u+\alpha)
\end{aligned}
$$

pour $u \neq \pm \alpha$ où chaque terme est localement intégrable et la majoration

$$
\left|f_{\alpha}(u+\mathrm{i} \varepsilon)\right| \leqslant\left(\frac{1}{2} \ln \left((\alpha+u)^{2}+1\right)+\pi\right)\left(\frac{1}{2} \ln \left((\alpha-u)^{2}+1\right)+\pi\right)
$$

pour $0<\varepsilon \leqslant 1$; d'après le théorème de la convergence dominée, on obtient l'égalité

$$
\begin{gathered}
\int_{\mathbb{R}} \phi(u+\beta) f_{\alpha}\left(u+\mathrm{i} 0_{+}\right) d u=C_{\phi}(\alpha, \beta)-\pi^{2} \int_{u \leqslant-|\alpha|} \phi(u+\beta) d u \\
+\mathrm{i} \pi \int_{\mathbb{R}} \phi(u+\beta) \mu(u-\alpha) \ln |u+\alpha| d u+\mathrm{i} \pi \int_{\mathbb{R}} \phi(u+\beta) \mu(u+\alpha) \ln |u-\alpha| d u .
\end{gathered}
$$

Cette relation est aussi valable pour la conjuguée de $\phi, \bar{\phi}$ et s'écrit

$$
\begin{aligned}
& \int_{\mathbb{R}} \bar{\phi}(u+\beta) f_{\alpha}\left(u+\mathrm{i} 0_{+}\right) d u=C_{\bar{\phi}}(\alpha, \beta)-\pi^{2} \int_{u \leqslant-|\alpha|} \bar{\phi}(u+\beta) d u \\
& \quad+\mathrm{i} \pi \int_{\mathbb{R}} \bar{\phi}(u+\beta) \mu(u-\alpha) \ln |u+\alpha| d u+\mathrm{i} \pi \int_{\mathbb{R}} \bar{\phi}(u+\beta) \mu(u+\alpha) \ln |u-\alpha| d u .
\end{aligned}
$$

En prenant le conjugué de chacun des termes de l'expression précédente et en remarquant que

$$
C_{\bar{\phi}}(\alpha, \beta)=\overline{C_{\phi}(\alpha, \beta)},
$$


on obtient

$$
\begin{aligned}
& \overline{\int_{\mathbb{R}} \bar{\phi}(u+\beta) f_{\alpha}\left(u+\mathrm{i} 0_{+}\right) d u}=C_{\phi}(\alpha, \beta)-\pi^{2} \int_{u \leqslant-|\alpha|} \phi(u+\beta) d u \\
&-\mathrm{i} \pi \int_{\mathbb{R}} \phi(u+\beta) \mu(u-\alpha) \ln |u+\alpha| d u-\mathrm{i} \pi \int_{\mathbb{R}} \phi(u+\beta) \mu(u+\alpha) \ln |u-\alpha| d u .
\end{aligned}
$$

En posant

$$
\Gamma(\alpha, \beta)=\int_{\mathbb{R}} \phi(u+\beta) f_{\alpha}\left(u+\mathrm{i} 0_{+}\right) d u+\overline{\int_{\mathbb{R}} \bar{\phi}(u+\beta) f_{\alpha}\left(u+\mathrm{i} 0_{+}\right) d u},
$$

la somme terme à terme des égalités (3.3.29) et (3.3.30) permet d'obtenir

$$
C_{\phi}(\alpha, \beta)=\Gamma(\alpha, \beta)+\pi^{2} \int_{u \leqslant-|\alpha|} \phi(u+\beta) d u .
$$

D'après la première partie de cette démonstration, $\Gamma$ est une fonction de classe $C^{d}$ sur $\mathbb{R}^{2}$. Comme $C_{\phi}$ et $\alpha \mapsto \int_{u \leqslant-|\alpha|} \phi(u+\beta) d u$ sont des fonctions paires en $\alpha, \Gamma$ est paire en $\alpha$.

LEMME 3.3.9. - On $a$ :

$$
\int_{\mathbb{R}} \frac{\ln |1+u|}{u(u+2)} d u=\frac{\pi^{2}}{2}
$$

Démonstration. - La relation $\frac{1}{u(u+2)}=\frac{1}{2}\left(\frac{1}{u}-\frac{1}{u+2}\right)$ permet d'obtenir :

$$
\begin{aligned}
\int_{\mathbb{R}} \frac{\ln |1+u|}{u(u+2)} d u & =\lim _{a \rightarrow \infty} \int_{|u| \leqslant a} \frac{\ln |1+u|}{u(u+2)} d u \\
& =\lim _{a \rightarrow \infty} \frac{1}{2} \int_{|u| \leqslant a} \frac{\ln |1+u|-\ln |1-u|}{u} d u .
\end{aligned}
$$

Par le changement de variable $u \mapsto 1 / u$, on a pour $0<1<a$ :

$$
\int_{1 \leqslant|u| \leqslant a} \frac{\ln |1+u|-\ln |1-u|}{u} d u=\int_{\frac{1}{a} \leqslant|u| \leqslant 1} \frac{\ln |1+u|-\ln |1-u|}{u} d u
$$

On en déduit l'égalité :

$$
\lim _{a \rightarrow \infty} \frac{1}{2} \int_{|u| \leqslant a} \frac{\ln |1+u|-\ln |1-u|}{u} d u=2 \int_{0}^{1} \frac{\ln (1+u)-\ln (1-u)}{u} d u .
$$

Comme

$$
\ln (1+u)-\ln (1-u)=2 \sum_{n \geqslant 0} \frac{u^{2 n+1}}{2 n+1}
$$

pour $0<u<1$, on obtient

$$
\int_{\mathbb{R}} \frac{\ln |1+u|}{u(u+2)} d u=\frac{\pi^{2}}{2}
$$


LEMME 3.3.10. - Soit $\phi \in \mathcal{C}_{c}^{d+1}(\mathbb{R})$. La fonction $A_{\phi}$ appartient à $\mathcal{C}^{d}(\{\alpha \neq 0\})$. De plus, on a les égalités

$$
\partial_{\beta} A_{\phi}=A_{\phi^{\prime}}
$$

et pour $n \leqslant d, \partial_{\alpha}^{n} A_{\phi}$ possède un développement asymptotique en $\alpha=0$ qui est égal à :

$$
\partial_{\alpha}^{n} A_{\phi}=\left\{\begin{array}{c}
-2 \operatorname{sign}(\alpha) \sum_{\substack{s=2 \\
s \text { pair }}}^{n}(s-1) ! \alpha^{-s} H_{\phi}^{(n+1-s)}(\beta)+2 \operatorname{sign}(\alpha) \ln |\alpha| H_{\phi}^{(n+1)}(\beta) \\
-\operatorname{sign}(\alpha)\left[J_{n} H_{\phi}^{(n+1)}(\beta)+2 \int_{\mathbb{R}} \ln |u|^{2} \phi^{(n+1)}(2 u+\beta) d u\right]+o(1) \\
\text { si n est impair, } \\
2 \operatorname{sign}(\alpha) \sum_{\substack{s=1 \\
\text { impair }}}^{n}(s-1) ! \alpha^{-s} H_{\phi}^{(n+1-s)}(\beta)+\pi^{2} \phi^{(n)}(\beta)+o(1) \\
\text { si n est pair. }
\end{array}\right.
$$

où o(1) représente une fonction continue sur $\mathbb{R}^{2}$ nulle pour $\alpha=0$.

Démonstration. - Sur l'ouvert $\{\alpha \neq 0\} \subset \mathbb{R}^{2}$, l'application

$$
(\alpha, \beta) \mapsto \int_{\mathbb{R}} \omega(u) \phi(2 \alpha u+\alpha+\beta) d u
$$

est de classe $C^{d}$, sa dérivée en $\beta$ est égale à

$$
\int_{\mathbb{R}} \omega(u) \phi^{\prime}(2 \alpha u+\alpha+\beta) d u
$$

et sa dérivée d'ordre $n$ en $\alpha$ s'écrit

$$
\begin{aligned}
& \text { 3.3.31) } \partial_{\alpha}^{n} \int_{\mathbb{R}} \omega(u) \phi(2 \alpha u+\alpha+\beta) d u=\int_{\mathbb{R}} \omega(u)(2 u+1)^{n} \phi^{(n)}(2 \alpha u+\alpha+\beta) d u \\
& =\sum_{q=1}^{n} 2^{q} \mathcal{C}_{n}^{q} \int_{\mathbb{R}} \ln |1+u| u^{q-1} \phi^{(n)}(2 \alpha u+\alpha+\beta) d u+\int_{\mathbb{R}} \omega(u) \phi^{(n)}(2 \alpha u+\alpha+\beta) d u .
\end{aligned}
$$

On étudie tout d'abord le second terme fourni par l'expression précédente suivant la parité de $n$.

Premier cas : $n$ est pair. Dans l'expression de la dérivée d'ordre $n$ en $\alpha$ de $A_{\phi}$ apparaît d'après l'égalité (3.3.31) l'expression

$$
\int_{\mathbb{R}} \omega(u) \phi^{(n)}(2 \alpha u+\alpha+\beta) d u+\int_{\mathbb{R}} \omega(u) \phi^{(n)}(-2 \alpha u-\alpha+\beta) d u .
$$

En utilisant le changement de variable $u \mapsto-u-2$ dans le deuxième terme de cette expression, celle-ci devient

$$
\int_{\mathbb{R}} \frac{\ln |1+u|}{u} \phi^{(n)}(2 \alpha u+\alpha+\beta) d u-\int_{\mathbb{R}} \frac{\ln |1+u|}{u+2} \phi^{(n)}(2 \alpha u+3 \alpha+\beta) d u
$$


puis,

$$
\begin{aligned}
& 2 \int_{\mathbb{R}} \frac{\ln |1+u|}{u(u+2)} \phi^{(n)}(2 \alpha u+\alpha+\beta) \\
& \quad+\int_{\mathbb{R}} \frac{\ln |1+u|}{u+2}\left(\phi^{(n)}(2 \alpha u+\alpha+\beta)-\phi^{(n)}(2 \alpha u+3 \alpha+\beta)\right) d u
\end{aligned}
$$

Comme $u \mapsto \frac{\ln |1+u|}{u(u+2)}$ est intégrable sur $\mathbb{R}$, la fonction

$$
(\alpha, \beta) \in \mathbb{R}^{2} \longmapsto 2 \int_{\mathbb{R}} \frac{\ln |1+u|}{u(u+2)} \phi^{(n)}(2 \alpha u+\alpha+\beta) d u
$$

est continue. Pour $\alpha=0$, la valeur de cette fonction est égale à

$$
2 \phi^{(n)}(\beta) \int_{\mathbb{R}} \frac{\ln |1+u|}{u(u+2)} d u .
$$

D'après le lemme (3.3.9), cette expression se simplifie ainsi

$$
\pi^{2} \phi^{(n)}(\beta) .
$$

Montrons que la fonction

$$
(\alpha, \beta) \longmapsto \int_{\mathbb{R}} \frac{\ln |1+u|}{u}\left(\phi^{(n)}(2 \alpha u+\alpha-\beta)-\phi^{(n)}(2 \alpha u+3 \alpha+\beta)\right) d u \quad(\alpha \neq 0)
$$

se prolonge en une fonction continue sur $\mathbb{R}^{2}$ nulle pour $\alpha=0$. Par le changement de variable $u \mapsto u / \alpha$, on obtient

$$
\begin{aligned}
\int_{\mathbb{R}} \frac{\ln |1+u|}{u}\left(\phi^{(n)}(2 \alpha u+\alpha+\beta)-\phi^{(n)}(2 \alpha u+3 \alpha+\beta)\right) d u \\
=\frac{1}{|\alpha|} \int_{\mathbb{R}} \frac{\ln \left|1+\frac{u}{\alpha}\right|}{\frac{u}{\alpha}+2}\left(\phi^{(n)}(2 u+\alpha+\beta)-\phi^{(n)}(2 u+3 \alpha+\beta)\right) d u
\end{aligned}
$$

Il existe une fonction $\eta$ continue et à support compact sur $\mathbb{R}$ telle que

$$
\left|\phi^{(n)}(x+2 \alpha)-\phi^{(n)}(x)\right| \leqslant|\alpha| \eta(x) \quad \forall x \in \mathbb{R}, \forall \alpha \in[-1,1] .
$$

On en déduit la majoration suivante

$$
\begin{aligned}
\frac{1}{|\alpha|} \mid \int_{\mathbb{R}} \frac{\ln \left|1+\frac{u}{\alpha}\right|}{\frac{u}{\alpha}+2}\left(\phi^{(n)}(2 u+\alpha+\beta)-\phi^{(n)}(2 u+3 \alpha\right. & +\beta)) d u \mid \\
& \leqslant \int_{\mathbb{R}}\left|\frac{\ln \left|1+\frac{u}{\alpha}\right|}{\frac{u}{\alpha}+2}\right| \eta(2 u+\beta) d u
\end{aligned}
$$

pour $|\alpha| \leqslant 1$. Soit $\mathcal{K}$ le support de la fonction $\eta$ et $\mathcal{K}^{\prime}$ une partie compacte de $\mathbb{R}$. On considère alors la partie compacte $\mathcal{K}^{\prime \prime}$ de $\mathbb{R}$ suivante

$$
\mathcal{K}^{\prime \prime}=\left\{\frac{\beta-k}{2} \mid k \in \mathcal{K}, \beta \in \mathcal{K}^{\prime}\right\} .
$$


Pour $|\alpha| \leqslant 1$ et $\beta \in \mathcal{K}^{\prime}$, on a

$$
\int_{\mathbb{R}}\left|\frac{\ln \left|1+\frac{u}{\alpha}\right|}{\frac{u}{\alpha}+2}\right| \eta(2 u+\beta) d u \leqslant \sup _{x \in \mathbb{R}}|\eta(x)| \int_{\mathcal{K}^{\prime \prime}}\left|\frac{\ln \left|1+\frac{u}{\alpha}\right|}{\frac{u}{\alpha}+2}\right| d u \rightarrow 0 \text { si } \alpha \rightarrow 0 .
$$

On en déduit que

$$
\int_{\mathbb{R}} \omega(u) \phi^{(n)}(2 \alpha u+\alpha+\beta) d u+\int_{\mathbb{R}} \omega(u) \phi^{(n)}(-2 \alpha u-\alpha+\beta) d u=\pi^{2} \phi^{(n)}(\beta)+o(1)
$$

où $o(1)$ est une fonction continue sur $\mathbb{R}^{2}$ nulle pour $\alpha=0$.

Deuxième cas : $n$ est impair. Dans l'expression de la dérivée d'ordre $n$ en $\alpha$ de $A_{\phi}$ apparaît d'après (3.3.31) l'expression

$$
\int_{\mathbb{R}} \omega(u) \phi^{(n)}(2 \alpha u+\alpha+\beta) d u-\int_{\mathbb{R}} \omega(u) \phi^{(n)}(-2 \alpha u-\alpha+\beta) d u .
$$

En utilisant le changement de variable $u \mapsto-u-1$ sur le second terme de l'expression précédente, celle-ci devient

$$
\int_{\mathbb{R}} \frac{\ln |1+u|}{u} \phi^{(n)}(2 \alpha u+\alpha+\beta) d u+\int_{\mathbb{R}} \frac{\ln |u|}{u+1} \phi^{(n)}(2 \alpha u+\alpha+\beta) d u
$$

puis en intégrant par partie,

$$
-2 \alpha \int_{\mathbb{R}} \ln |1+u| \ln |u| \phi^{(n+1)}(2 \alpha u+\alpha+\beta) d u
$$

et enfin en posant le changement de variable $u \mapsto u / \alpha$,

$$
\begin{aligned}
-2 \operatorname{sign}(\alpha) \int_{\mathbb{R}} \ln |u| \ln |u+\alpha| \phi^{(n+1)}(2 \mathfrak{u}+\alpha+\beta) d u \\
\quad+2 \operatorname{sign}(\alpha) \ln |\alpha| \int_{\mathbb{R}} \ln |u+\alpha| \phi^{(n+1)}(2 u+\alpha+\beta) d u \\
\quad+2 \operatorname{sign}(\alpha) \ln |\alpha| \int_{\mathbb{R}} \ln |u| \phi^{(n+1)}(2 u+\alpha+\beta) d u .
\end{aligned}
$$

On observe que l'on a le développement asymptotique suivant

$$
\begin{aligned}
& 2 \operatorname{sign}(\alpha) \ln |\alpha| \int_{\mathbb{R}} \ln |u+\alpha| \phi^{(n+1)}(2 u+\alpha+\beta) \\
&+2 \operatorname{sign}(\alpha) \ln |\alpha| \int_{\mathbb{R}} \ln |u| \phi^{(n+1)}(2 u+\alpha+\beta) d u \\
& \quad=2 \operatorname{sign}(\alpha) \ln |\alpha| H_{\phi}^{(n+1)}(\beta)+o(1)
\end{aligned}
$$

ou $o(1)$ représente une fonction continue sur $\mathbb{R}^{2}$ nulle pour $\alpha=0$. Le changement de variable $u \mapsto u+\alpha / 2$ permet d'obtenir

$$
\int_{\mathbb{R}} \ln |u| \ln |u+\alpha| \phi^{(n+1)}(2 u+\alpha+\beta) d u=C_{\psi}\left(\frac{\alpha}{2}, \frac{\beta}{2}\right)
$$

où

$$
\psi(u)=\phi^{(n+1)}(2 u)
$$


D'après le lemme $3.3 .8, C_{\psi}$ se prolonge continûment sur $\mathbb{R}^{2}$ ainsi le développement asymptotique de l'expression (3.3.32) s'écrit

$$
-2 \operatorname{sign}(\alpha) \int_{\mathbb{R}} \ln |u|^{2} \phi^{(n+1)}(2 u+\beta) d u+2 \operatorname{sign}(\alpha) \ln |\alpha| H_{\phi}^{(n+1)}(\beta)+o(1)
$$

où $o(1)$ est une fonction continue sur $\mathbb{R}^{2}$ nulle pour $\alpha=0$. À présent, on étudie le premier terme de (3.3.31).

Pour $1 \leqslant q \leqslant n$, par le changement de variable $u \leftarrow \alpha(u+1)$, on a l'égalité :

$$
\begin{aligned}
& \int_{\mathbb{R}} \ln |1+u| u^{q-1} \phi^{(n)}(2 \alpha u+\alpha+\beta) d u \\
& =\operatorname{sign}(\alpha) \sum_{r=0}^{q-1}(-1)^{q-1-r} \int_{\mathbb{R}}(\ln |u|-\ln |\alpha|) \mathcal{C}_{q-1}^{r} \frac{u^{r}}{\alpha^{r+1}} \phi^{(n)}(2 u-\alpha+\beta) d u
\end{aligned}
$$

Pour $r<n$, en intégrant par parties, on a l'égalité

$$
\int_{\mathbb{R}} u^{r} \phi^{(n)}(2 u-\alpha+\beta) d u=0,
$$

ainsi, on a

$$
\int_{\mathbb{R}} \ln |u| u^{r} \phi^{(n)}(2 u-\alpha+\beta) d u=\frac{1}{2^{r+1}} \int_{\mathbb{R}} \ln |u| u^{r} \phi^{(n)}(u-\alpha+\beta) .
$$

Puis, pour $r \geqslant 1$, en intégrant par partie, on a :

$$
\int_{\mathbb{R}} \ln |u| u^{r} \phi^{(n)}(u-\alpha+\beta) d u=-r \int_{\mathbb{R}} \ln |u| u^{r} \phi^{(n-1)}(u-\alpha+\beta) d u ;
$$

d'où par récurrence pour $r \geqslant 0$, avec $r<n$, on obtient

$$
\int_{\mathbb{R}} \ln |u| u^{r} \phi^{(n)}(u-\alpha+\beta) d u=r !(-1)^{r} \int_{\mathbb{R}} \ln |u| \phi^{(n-r)}(u-\alpha+\beta) d u .
$$

Ensuite le développement de Taylor en $\alpha$ à l'ordre $r+1$ de $\phi^{(n-r)}(u-\alpha+\beta)$ est

$$
\phi^{(n-r)}(u-\alpha+\beta)=\sum_{k=0}^{r+1} \frac{(-1)^{k}}{k !} \alpha^{k} \phi^{(n+k-r)}(u+\beta)+o\left(\alpha^{r+1}\right)
$$

où $o\left(\alpha^{r+1}\right)$ représente le produit d'une fonction continue sur $\mathbb{R}^{2}$ nulle pour $\alpha=0$ multipliée par $\alpha^{r+1}$. Les égalités (3.3.34), (3.3.35) et (3.3.36) permettent d'obtenir le développement asymptotique suivant de (3.3.33)

$$
\operatorname{sign}(\alpha) \sum_{r=0}^{q-1}\left(\frac{(-1)^{q-1} \mathcal{C}_{q-1}^{r} r !}{2^{r+1}} \sum_{k=0}^{r+1} \alpha^{k-r-1} \frac{(-1)^{k}}{k !} \int_{\mathbb{R}} \ln |u| \phi^{(n-r)}(u+\beta) d u\right)+o(1) .
$$

Cette expression s'écrit aussi

$$
\operatorname{sign}(\alpha) \sum_{s=0}^{q}\left(\alpha^{-s} \sum_{r=\max (s-1,0)}^{q-1} \frac{(-1)^{q-s+r} \mathcal{C}_{q-1}^{r} r !}{(r-s+1) ! 2^{r+1}} \int_{\mathbb{R}} \ln |u| \phi^{(n-r)}(u+\beta) d u\right)+o(1) .
$$


On en déduit le développement asymptotique du premier terme de l'expression (3.3.31) :

$$
\operatorname{sign}(\alpha) \sum_{s=0}^{n} \alpha^{-s} \sum_{q=\max (s, 1)}^{n}\left[2^{q} \mathcal{C}_{n}^{q}\left(\sum_{r=\max (s-1,0)}^{q-1} \frac{(-1)^{q-s+r} \mathcal{C}_{q-1}^{r} r !}{(r-s+1) ! 2^{r+1}}\right)\right] H_{\phi}^{(n-s+1)}(\beta)
$$

Calculons les coefficients de ce développement.

On suppose tout d'abord $s \geqslant 1$, le coefficient s'exprime alors ainsi :

$$
\begin{aligned}
& \sum_{q=s}^{n} 2^{q} \mathcal{C}_{n}^{q}\left(\sum_{r=s-1}^{q-1} \frac{(-1)^{q-s+r} \mathcal{C}_{q-1}^{r} r !}{(r-s+1) ! 2^{r+1}}\right) \\
&=\sum_{q=0}^{n-s} \frac{n !}{(n-q-s) !(q+s)}\left(\sum_{r=0}^{q} \frac{(-1)^{q+s+r+1} 2^{q-r}}{(q-r) ! r !}\right) .
\end{aligned}
$$

On obtient en exprimant de deux manières différentes le $q^{i e m e}$ terme du développement en série entière de $e^{X} e^{-2 X}$, l'égalité suivante :

$$
\sum_{r=0}^{q} \frac{(-1)^{q+s+r+1} 2^{q-r}}{(q-r) ! r !}=\frac{(-1)^{q+s+1}}{q !} .
$$

On est donc ramené à calculer :

$$
(-1)^{s+1} n ! \sum_{q=0}^{n-s} \frac{(-1)^{q}}{(n-q-s) !(q+s) q !} .
$$

De même,

$$
\sum_{q=0}^{n-s} \frac{(-1)^{q}}{(n-q-s) !(q+s) q !}
$$

représente le $n^{\text {ieme }}$ coefficient du développement en série entière de

$$
e^{X} \int X^{s-1} e^{-X} d X
$$

où la primitive est prise nulle en 0 . Pour $q \in \mathbb{N}$, posons

$$
\theta_{q}=e^{X} \int X^{q} e^{-X} d X
$$

En intégrant par partie, on obtient pour $q \geqslant 1$ la relation

$$
-\theta_{q}+q \theta_{q-1}=X^{q}
$$

ainsi pour $n>q$, on a

$$
\theta_{q}^{(n)}(0)=q \theta_{q-1}^{(n)}(0)
$$

Ensuite, comme la primitive dans la définition de $\theta_{q}$ est prise nulle en 0 , on a

$$
\theta_{0}=e^{X}-1 \text { et } \theta_{0}^{(n)}=\frac{1}{n !}
$$


pour $n \geqslant 1$. On en déduit l'égalité suivante

$$
\theta_{q}^{(n)}(0)=\frac{q !}{n !}
$$

pour $q<n$. L'égalité entre les deux expressions du $n^{i e m e}$ coefficient du développement de $\theta_{s-1}$ permet d'obtenir l'égalité :

$$
(-1)^{s+1} n ! \sum_{q=0}^{n-s} \frac{(-1)^{q}}{(n-q-s) !(q+s) q !}=(-1)^{s+1}(s-1) ! .
$$

Pour $s=0$, l'égalité (3.3.37) montre que le coefficient est égale à :

$$
n ! \sum_{q=0}^{n-1} \frac{(-1)^{q+1} 2^{q+1}}{(n-q-1) !(q+1)(q+1) !}=-\int_{-2}^{0} \frac{(1+x)^{n}-1}{x} d x=-J_{n} .
$$

Pour $n$ impair (resp. $n$ pair), $\partial_{\alpha}^{n} A_{\phi}$ est une fonction impaire (resp. paire) en $\alpha$, ainsi seules les fonctions $\operatorname{sign}(\alpha) \alpha^{s}$ avec $s$ pair (resp. $s$ impair) interviennent dans son développement asymptotique. On en déduit le résultat.

LEMME 3.3.11. - Soit $\phi \in \mathcal{C}_{c}^{d+1}(\mathbb{R})$. La fonction $B_{\phi}$ est de classe $C^{d}$ sur l'ouvert $\{\alpha \neq 0\} \subset \mathbb{R}^{2}$. On a l'équation

$$
\partial_{\beta} B_{\phi}=B_{\phi^{\prime}}
$$

et $\partial_{\alpha}^{n} B_{\phi}$ possède un développement asymptotique en $\alpha=0$ qui est égal à :

$$
\partial_{\alpha}^{n} B_{\phi}=\left\{\begin{array}{cc}
2 \ln |2 \alpha| H_{\phi}^{(n+1)}(\beta)-2 \sum_{\substack{s=2 \\
s \text { pair }\\
}}^{n}(s-1) ! \alpha^{-s} H_{\phi}^{(n+1-s)}(\beta) \\
+2 I_{n} H_{\phi}^{(n+1)}(\beta)+o(1) & \text { si } n \text { est impair }, \\
2 \sum_{\substack{s=1 \\
\text { impair }}}^{n}(s-1) ! \alpha^{-s} H_{\phi}^{(n+1-s)}(\beta)+o(1) & \text { si } n \text { est pair. }
\end{array}\right.
$$

pour $n \leqslant d$ où o(1) représente une fonction continue sur $\mathbb{R}^{2}$ nulle pour $\alpha=0$.

Démonstration. - La fonction $H_{\phi}$ est de classe $C^{d+1}$ sur $\mathbb{R}$ et pour $a, b \in \mathbb{N}$ tels que $a+b \leqslant d+1$ le développement de Taylor de $H_{\phi}^{(a)}(\beta+\alpha)$ à l'ordre $b$ en $\alpha$ s'écrit

$$
H_{\phi}^{(a)}(\beta+\alpha)=\sum_{t=0}^{b} \frac{\alpha^{t}}{t !} H_{\phi}^{(a+t)}(\beta)+o\left(\alpha^{b}\right)
$$


où $o\left(\alpha^{b}\right)$ représente le produit d'une fonction continue sur $\mathbb{R}^{2}$ nulle pour $\alpha=0$ par $\alpha^{b}$. En utilisant les développements de Taylor ci-dessus, on obtient

$$
\begin{aligned}
& \partial_{\alpha}^{n}\left(\ln |2 \alpha| H_{\phi}^{\prime}(\beta+\alpha)\right) \\
& =\ln |2 \alpha| H_{\phi}^{(n+1)}(\beta)+\sum_{p=1}^{n} \frac{(-1)^{p-1} \mathcal{C}_{n}^{p}(p-1) !}{\alpha^{p}}\left[\sum_{q=0}^{p} \frac{\alpha^{q}}{q !} H_{\phi}^{(n-p+q+1)}(\beta)\right]+o(1) \\
& =\ln |2 \alpha| H_{\phi}^{(n+1)}(\beta)+\sum_{s=0}^{n}\left[\sum_{p=\max (s, 1)}^{n} \frac{(-1)^{p-1}(p-1) ! \mathcal{C}_{n}^{p}}{(p-s) !}\right] \alpha^{-s} H_{\phi}^{n+1-s}(\beta)+o(1)
\end{aligned}
$$

où $o(1)$ représente une fonction continue sur $\mathbb{R}^{2}$ nulle pour $\alpha=0$. Calculons le coefficient entre crochet. En supposant $s \geqslant 1$, on a

$$
\begin{aligned}
\sum_{p=s}^{n} \frac{(-1)^{p-1}(p-1) ! \mathcal{C}_{n}^{p}}{(p-s) !} & =\frac{n !}{(n-s) !} \sum_{p=0}^{n-s} \mathcal{C}_{n-s}^{p} \frac{(-1)^{p+s-1}}{p+s} \\
& =\frac{n !}{(n-s) !} \int_{-1}^{0}(1+u)^{n-s} u^{s-1} d u
\end{aligned}
$$

On montre que

$$
\int_{-1}^{0}(1+u)^{n-s} u^{s-1} d u=\frac{(-1)^{s+1}}{s \mathcal{C}_{n}^{s}}
$$

ainsi

$$
\sum_{p=s}^{n} \frac{(-1)^{p-1}(p-1) ! \mathcal{C}_{n}^{p}}{(p-s) !}=(-1)^{s+1}(s-1) !
$$

Pour $s=0$, on a :

$$
\sum_{p=1}^{n} \frac{(-1)^{p-1} \mathcal{C}_{n}^{p}}{p}=\int_{-1}^{0} \frac{(1+x)^{n}-1}{x} d x=I_{n}
$$

Pour obtenir le développement asymptotique recherché, il reste à considérer les puissances paires (resp. impaires) de $\alpha$ si $n$ est impair (resp. pair) ainsi pour $n$ est impair, comme $B_{\phi}$ est impaire d'après (3.3.38), on a

$\partial_{\alpha}^{n} B_{\phi}=2 \ln |2 \alpha| H_{\phi}^{(n+1)}(\beta)-2 \sum_{\substack{s=2 \\ s \text { pair }}}^{n}(s-1) ! \alpha^{-s} H_{\phi}^{(n+1-s)}(\beta)+2 I_{n} H_{\phi}^{(n+1)}(\beta)+o(1)$.

Pour $n$ pair, on obtient

$$
\partial_{\alpha}^{n} B_{\phi}=2 \sum_{\substack{s=1 \\ s \text { impair }}}^{n}(s-1) ! \alpha^{-s} H_{\phi}^{(n+1-s)}(\beta)+o(1) .
$$


Lemme 3.3.12. - Pour $\phi \in \mathcal{C}_{c}^{d+1}(\mathbb{R})$, on a les propriétés suivantes :

(1) La fonction

$$
\operatorname{sign}(\alpha) A_{\phi}-B_{\phi}
$$

appartient à $\mathcal{H}(\{\alpha \neq 0\}, d)$.

(2) On a l'équation

$$
\partial_{\beta}\left(\operatorname{sign}(\alpha) A_{\phi}-B_{\phi}\right)=\operatorname{sign}(\alpha) A_{\phi^{\prime}}-B_{\phi^{\prime}}
$$

(3) On a

$$
\operatorname{sign}(\alpha) \partial_{\alpha}^{n} A_{\phi}-\partial_{\alpha}^{n} B_{\phi}=\left\{\begin{array}{lc}
\text { une fonction continue sur } \mathbb{R}^{2} & \text { si } n \text { est impair } \\
\operatorname{sign}(\alpha) \pi^{2} \phi^{(n)}(\beta) \text { modulo une fonction continue sur } \mathbb{R}^{2} & \text { si } n \text { est pair }
\end{array}\right.
$$

pour $n \leqslant d$.

Démonstration. — Cela découle des lemmes 3.3.10 et 3.3.11. Pour $n$ impair, on obtient

$$
\begin{aligned}
& \operatorname{sign}(\alpha) \partial_{\alpha}^{n} A_{\phi}-\partial_{\alpha}^{n} B_{\phi} \\
& \quad=\left(2 I_{n}+2 \ln |2|-J_{n}\right) H_{\phi}^{(n+1)}(\beta)-2 \int_{\mathbb{R}} \ln |u|^{2} \phi^{(n+1)}(2 u+\beta) d u+o(1) .
\end{aligned}
$$

Pour $n$ pair, on a

$$
\operatorname{sign}(\alpha) \partial_{\alpha}^{n} A_{\phi}-\partial_{\alpha}^{n} B_{\phi}=\operatorname{sign}(\alpha) \pi^{2} \phi^{(n)}(\beta)+o(1) .
$$

\subsection{Propriétés de Chc pour $G$ non compact}

Grâce aux résultats de la section précédente, on peut calculer les termes du corollaire 2.2.5. Tout d'abord, on s'intéresse aux termes associés à $\mathfrak{h}_{\mathcal{S}}$ dans la première sous-section puis aux termes associés à $\mathfrak{h}_{\varnothing}$.

DÉfinition 3.4.1. - Pour $d \in \mathbb{N}$, on considère l'espace de fonction $\mathcal{W}_{c}\left(\mathfrak{h}_{\varnothing}, d\right)$ constitué des fonctions $\phi$ de $\mathcal{H}_{c}\left(\mathfrak{h}_{\varnothing}^{\text {reg }}, d\right)$ telles que

$$
\left(\partial_{1}-\partial_{2}\right)^{p}\left(\partial_{1}+\partial_{2}\right)^{q} \phi
$$

se prolonge en une fonction continue sur $\mathfrak{h}_{\varnothing}$ si $p$ est impair et $\mathcal{W}_{c}\left(\mathfrak{h}_{\mathcal{S}}, d\right)$ le sous-espace des fonctions de $\mathcal{C}_{c}^{d}\left(\mathfrak{h}_{\mathcal{S}}\right)$ paire en $u$.

Remarque. - Pour $\phi \in D(\mathfrak{g})$, on a $\phi_{\varnothing} \in \mathcal{W}_{c}\left(\mathfrak{h}_{\varnothing}, d\right)$ et $\phi_{\mathcal{S}} \in \mathcal{W}_{c}\left(\mathfrak{h}_{\mathcal{S}}\right)$. 
3.4.1. Les termes associés à $\mathfrak{h}_{\mathcal{S}}$. - On a $\mathcal{S}=\left\{e_{1}-e_{2}\right\}$ et $\mathbf{e}_{\mathcal{S}}(x)=-2 \cdot u$, ainsi :

$$
\mathfrak{h}_{\mathcal{S}}^{+}=\left\{\left(\begin{array}{cc}
\mathrm{i} v & u \\
u & \mathrm{i} v
\end{array}\right) \mid u<0 \text { et } v \in \mathbb{R}\right\} \text { et } y_{\mathcal{S}}^{L}=0 \forall L .
$$

Soit $\psi \in \mathcal{D}(\mathfrak{g})$. On identifie $\mathfrak{h}_{\mathcal{S}}$ avec $\mathbb{C}$ par l'application $\left(\begin{array}{cc}\mathrm{i} v & u \\ u & i v\end{array}\right) \mapsto v+\mathrm{i} u$ ainsi $\mathfrak{h}_{\mathcal{S}}^{+}$ s'identifie à

$$
P_{-}=\{z \in \mathbb{C} \mid \Im(z)<0\} .
$$

On rappelle que $P_{+}$désigne le demi-plan de Poincaré $\{z \in \mathbb{C} \mid \Im(z)>0\}$. On note $d z=d u d v$ la mesure produit sur $\mathbb{C}$, ln la détermination principale du logarithme, Arg la fonction argument associée et $\mu$ la fonction définie sur $\mathbb{R}$ par $\mu(x)=0$ si $x \geqslant 0$ et 1 sinon. Avec l'identification de $\mathfrak{h}_{\mathcal{S}}$ avec $\mathbb{C}, \psi_{\mathcal{S}}$ vérifie

$$
\psi_{\mathcal{S}}(z)=\psi_{\mathcal{S}}(\bar{z}) .
$$

On a l'égalité

$$
\begin{aligned}
\int_{C_{\mathcal{S}}^{i d}(t)} \nu_{i d, x^{\prime}, \mathcal{S}}+ & \int_{C_{\mathcal{S}}^{(1,2)}(t)} \nu_{(1,2), x^{\prime}, \mathcal{S}} \\
& =-2 \int_{P_{-}} \frac{\psi_{\mathcal{S}}(z)}{\left(\bar{z}-x_{1}^{\prime}\right)\left(z-x_{2}^{\prime}\right)} d z+2 \int_{P_{-}} \frac{\psi_{\mathcal{S}}(z)}{\left(z-x_{1}^{\prime}\right)\left(\bar{z}-x_{2}^{\prime}\right)} d z .
\end{aligned}
$$

\section{REMARques}

(1) Comme $y_{\mathcal{S}}^{L}=0$ pour $L=i d$ et $(1,2)$, l'expression de gauche ci-dessus est indépendante de $t$; on omettra le $t$ dans la suite de cette sous-section.

(2) Le facteur 2 de l'expression de droite provient de la normalisation des mesures.

DÉfinition 3.4.2. - On considère pour $\phi \in \mathcal{W}_{c}\left(\mathfrak{h}_{\mathcal{S}}, d\right)$, la fonction définie sur $\mathfrak{h}_{\varnothing}^{\text {rreg }}$ par

$$
\widetilde{\mathcal{B}}(\phi)\left(x^{\prime}\right)=-2 \int_{P_{-}} \frac{\phi(z)}{\left(\bar{z}-x_{1}^{\prime}\right)\left(z-x_{2}^{\prime}\right)} d z+2 \int_{P_{-}} \frac{\phi(z)}{\left(z-x_{1}^{\prime}\right)\left(\bar{z}-x_{2}^{\prime}\right)} d z .
$$

L'expression précédente s'écrit grâce à l'égalité (3.4.1) qui est vérifiée pour $\phi$

$$
-2 \int_{P_{+}} \frac{\phi(z)}{\left(z-x_{1}^{\prime}\right)\left(\bar{z}-x_{2}^{\prime}\right)} d z+2 \int_{P_{+}} \frac{\phi(z)}{\left(\bar{z}-x_{1}^{\prime}\right)\left(z-x_{2}^{\prime}\right)} d z
$$

ainsi, on a

$$
\widetilde{\mathcal{B}}(\phi)\left(x^{\prime}\right)=-4 \mathrm{i} \cdot \int_{P_{+}} \Im\left(\frac{1}{\left(z-x_{1}^{\prime}\right)\left(\bar{z}-x_{2}^{\prime}\right)}\right) \phi(z) d z .
$$

Lemme 3.4.3. - Soit $\phi \in \mathcal{C}_{c}^{d+2}(\mathbb{C})$ telle que

$$
\phi(z)=\phi(\bar{z})
$$


pour $z \in \mathbb{C}$, alors il existe une fonction $B$ de classe $C^{d}$ sur $\mathbb{R}^{2}$ et antisymétrique ${ }^{(4)}$ telle que

$$
\int_{P_{+}} \Im\left(\frac{1}{\left(z-x_{1}^{\prime}\right)\left(\bar{z}-x_{2}^{\prime}\right)}\right) \phi(z) d z=\frac{\operatorname{sign}\left(x_{2}^{\prime}-x_{1}^{\prime}\right) \pi^{2}}{2} \phi\left(\min \left(x_{1}^{\prime}, x_{2}^{\prime}\right)\right)+B\left(x_{1}^{\prime}, x_{2}^{\prime}\right) .
$$

Démonstration. - On pose $\Gamma(\rho, \theta)=\phi\left(x_{1}^{\prime}+\rho e^{\mathrm{i} \theta}\right)$. En utilisant le changement de variable $z=x_{1}^{\prime}+\rho e^{\mathrm{i} \theta}$, on a :

$$
\int_{P_{+}} \frac{\phi(z)}{\left(z-x_{1}^{\prime}\right)\left(\bar{z}-x_{2}^{\prime}\right)} d z=\int_{\left.\mathbb{R}_{+} \times\right] 0, \pi[} \frac{\Gamma(\rho, \theta)}{e^{\mathrm{i} \theta}\left(\rho e^{-\mathrm{i} \theta}+x_{1}^{\prime}-x_{2}^{\prime}\right)} d \theta d \rho .
$$

Puis en intégrant par partie en $\rho$, on obtient :

$$
\begin{gathered}
\int_{0}^{\pi}\left[\Gamma(\rho, \theta) \ln \left(\rho e^{-\mathrm{i} \theta}+x_{1}^{\prime}-x_{2}^{\prime}\right)\right]_{0}^{\infty} d \theta \\
-\int_{\left.\mathbb{R}_{+} \times\right] 0, \pi[} \partial_{\rho} \Gamma(\rho, \theta) \ln \left(\rho e^{-\mathrm{i} \theta}+x_{1}^{\prime}-x_{2}^{\prime}\right) d \theta d \rho \\
=-\pi \ln \left|x_{2}^{\prime}-x_{1}^{\prime}\right| \phi\left(x_{1}^{\prime}\right)+\mathrm{i} \pi^{2} \mu\left(x_{1}^{\prime}-x_{2}^{\prime}\right) \phi\left(x_{1}^{\prime}\right) \\
\quad-\int_{\left.\mathbb{R}_{+} \times\right] 0, \pi[} \partial_{\rho} \Gamma(\rho, \theta) \ln \left(\rho e^{-\mathrm{i} \theta}+x_{1}^{\prime}-x_{2}^{\prime}\right) d \theta d \rho
\end{gathered}
$$

On remarque que

$$
\begin{aligned}
\int_{\left.\mathbb{R}_{+} \times\right] 0, \pi[} \partial_{\rho} \Gamma(\rho, \theta) & \operatorname{Arg}\left(\rho e^{-\mathrm{i} \theta}+x_{1}^{\prime}-x_{2}^{\prime}\right) d \theta d \rho \\
= & -\int_{\left.\mathbb{R}_{+} \times\right] 0, \pi[} \partial_{\rho} \Gamma(\rho, \theta) \ln \left(\rho e^{\mathrm{i} \theta}+x_{1}^{\prime}-x_{2}^{\prime}\right) d \theta d \rho \\
& =-\Xi_{\phi}\left(x_{1}^{\prime}, x_{2}^{\prime}\right) \text { (Définition 3.3.1); }
\end{aligned}
$$

ainsi

$$
\int_{P_{+}} \Im\left(\frac{1}{\left(z-x_{1}^{\prime}\right)\left(\bar{z}-x_{2}^{\prime}\right)}\right) \phi(z) d z=\pi^{2} \mu\left(x_{1}^{\prime}-x_{2}^{\prime}\right) \phi\left(x_{1}^{\prime}\right)+\Xi_{\phi}\left(x_{1}^{\prime}, x_{2}^{\prime}\right) .
$$

Ensuite, on a la relation :

$$
\int_{P_{+}} \Im\left(\frac{1}{\left(z-x_{1}^{\prime}\right)\left(\bar{z}-x_{2}^{\prime}\right)}\right) \phi(z) d z=-\int_{P_{+}} \Im\left(\frac{1}{\left(\bar{z}-x_{1}^{\prime}\right)\left(z-x_{2}^{\prime}\right)}\right) \phi(z) d z .
$$

On en déduit la nouvelle expression suivante :

$$
\int_{P_{+}} \Im\left(\frac{1}{\left(z-x_{1}^{\prime}\right)\left(\bar{z}-x_{2}^{\prime}\right)}\right) \phi(z) d z=-\pi^{2} \mu\left(x_{2}^{\prime}-x_{1}^{\prime}\right) \phi\left(x_{2}^{\prime}\right)-\Xi_{\phi}\left(x_{2}^{\prime}, x_{1}^{\prime}\right) .
$$

Avec les égalités (3.4.5) et (3.4.6), on obtient :

$$
\int_{P_{+}} \Im\left(\frac{1}{\left(z-x_{1}^{\prime}\right)\left(\bar{z}-x_{2}^{\prime}\right)}\right) \phi(z) d z=\frac{\pi^{2}}{2} \operatorname{sign}\left(x_{2}^{\prime}-x_{1}^{\prime}\right) \phi\left(\min \left(x_{1}^{\prime}, x_{2}^{\prime}\right)\right)-\frac{\widehat{\Xi}_{\phi}\left(x_{1}^{\prime}, x_{2}^{\prime}\right)}{2}
$$

$\overline{(4) \text { i.e. } B\left(x_{1}^{\prime}, x_{2}^{\prime}\right)}=-B\left(x_{2}^{\prime}, x_{1}^{\prime}\right)$ 
où $\widehat{\Xi}_{\phi}\left(x_{1}^{\prime}, x_{2}^{\prime}\right)=\Xi_{\phi}\left(x_{1}^{\prime}, x_{2}^{\prime}\right)-\Xi_{\phi}\left(x_{2}^{\prime}, x_{1}^{\prime}\right)$ (Définition 3.3.1). D'après le lemme 3.3.2, la fonction $\widehat{\Xi}_{\phi}$ est de classe $C^{d}$, on en déduit le résultat.

Proposition 3.4.4. - Soit $\phi \in \mathcal{W}_{c}\left(\mathfrak{h}_{\mathcal{S}}, d+2\right)$. Il existe une fonction $D$ de classe $C^{d}$ sur $\mathbb{R}^{2}$ et antisymétrique telle que :

$$
\widetilde{\mathcal{B}}(\phi)\left(x^{\prime}\right)=-2 \mathrm{i} \pi^{2} \operatorname{sign}(\alpha) \phi(0, \beta-|\alpha|)+D\left(x_{1}^{\prime}, x_{2}^{\prime}\right) .
$$

Démonstration. - Cela découle directement du lemme précédent et de l'égalité (3.4.3).

COROLlaire 3.4.5. - On a en particulier les propriétés suivantes :

(1) La fonction $\widetilde{\mathcal{B}}(\phi)$ appartient à $\mathcal{H}\left(\mathfrak{h}_{\varnothing}^{\text {'reg }}, d\right)$. Pour tout opérateur différentiel $w$ de degré au plus $d$, on peut définir $\langle\partial(w) \widetilde{\mathcal{B}}(\phi)\rangle$.

(2) On a l'équation

$$
\partial_{\beta} \widetilde{\mathcal{B}}(\phi)=\widetilde{\mathcal{B}}\left(\partial_{z} \phi\right)
$$

(3) Pour $n \leqslant d$, on $a$

$$
\left\langle\partial_{\alpha}^{n} \widetilde{\mathcal{B}}(\phi)\right\rangle= \begin{cases}0 & \text { si } n \text { est impair } \\ 4 \mathrm{i} \pi^{2} \partial_{v}^{n} \phi(0, \beta) & \text { si } n \text { est pair. }\end{cases}
$$

3.4.2. Calcul des termes associés à $\mathfrak{h}_{\varnothing}$ - - Par hypothèse, on a $\operatorname{sign}\left(V_{1}\right)=1$ et $\operatorname{sign}\left(V_{2}\right)=\operatorname{sign}\left(V_{2}^{\prime}\right)=-1$ ainsi, en posant $\varepsilon=\operatorname{sign}\left(V_{1}^{\prime}\right)$, on a :

$$
y^{i d}=\left(\begin{array}{l}
\varepsilon \\
1
\end{array}\right) \text { et } y^{(1,2)}=\left(\begin{array}{l}
-1 \\
-\varepsilon
\end{array}\right) ;
$$

comme $\mathfrak{h}_{\varnothing}$ est une sous-algèbre de Cartan compacte, $y_{\varnothing}^{L}=y^{L}$ pour $L=i d$ ou $(1,2)$. La transformée de Cayley vérifie :

$$
c_{\mathcal{S}}\left(\left(\begin{array}{cc}
\mathrm{i}(\beta+\alpha) & 0 \\
0 & \mathrm{i}(\beta-\alpha)
\end{array}\right)\right)=\left(\begin{array}{ll}
\mathrm{i} \beta & \mathrm{i} \alpha \\
\mathrm{i} \alpha & \mathrm{i} \beta
\end{array}\right) .
$$

D'après les propriétés des intégrales invariantes, $\left\langle\partial(w) \psi_{\varnothing}\right\rangle$ avec $w \in \operatorname{Sym}\left(\mathfrak{h}_{\varnothing, \mathbb{C}}\right)$ est une fonction définie sur le centre de $\mathfrak{g}$. Pour $w \in S y m\left(\mathfrak{h}_{\varnothing, \mathbb{C}}\right)$, la relation de saut s'écrit :

$$
\left\langle\partial(w) \psi_{\varnothing}\right\rangle(x)=\mathrm{i} \partial\left(c_{\mathcal{S}}(w)\right) \psi_{\mathcal{S}}(0, x) .
$$

Cela montre en particulier que $\left\langle\partial(w) \psi_{\varnothing}\right\rangle$ est une fonction lisse sur $\mathbb{R}$. On a l'égalité

$$
\begin{aligned}
& \int_{C_{\varnothing}^{i d}(t)} \nu_{i d, x^{\prime}, \varnothing}+\int_{C_{\varnothing}^{(1,2)}(t)} \nu_{(1,2), x^{\prime}, \varnothing} \\
& =\int_{\mathbb{R}^{2}}\left\{-\frac{1}{\left(x_{1}+\mathrm{i} \varepsilon t-x_{1}^{\prime}\right)\left(x_{2}+\mathrm{i} t-x_{2}^{\prime}\right)}+\frac{1}{\left(x_{2}-\mathrm{i} \varepsilon t-x_{1}^{\prime}\right)\left(x_{1}-\mathrm{i} t-x_{2}^{\prime}\right)}\right\} \psi_{\varnothing}(x) d x
\end{aligned}
$$


On pose pour $t>0, x^{\prime} \in \mathfrak{h}_{\varnothing}^{\text {/reg }}$ et $\phi \in \mathcal{W}_{c}\left(\mathfrak{h}_{\varnothing}, d\right)$

$$
\begin{aligned}
\widetilde{\mathcal{A}}^{t}(\phi) & \left(x^{\prime}\right) \\
= & \int_{\mathbb{R}^{2}}\left\{-\frac{1}{\left(x_{1}+\mathrm{i} \varepsilon t-x_{1}^{\prime}\right)\left(x_{2}+\mathrm{i} t-x_{2}^{\prime}\right)}+\frac{1}{\left(x_{2}-\mathrm{i} \varepsilon t-x_{1}^{\prime}\right)\left(x_{1}-\mathrm{i} t-x_{2}^{\prime}\right)}\right\} \phi(x) d x
\end{aligned}
$$

On a le premier résultat suivant pour les termes du corollaire 2.2.5 associés à $\mathfrak{h}_{\varnothing}$.

Lemme 3.4.6. - Pour $x^{\prime} \in \mathfrak{h}_{\varnothing}^{\text {'reg }}$ et $\phi \in \mathcal{W}_{c}\left(\mathfrak{h}_{\varnothing}, d\right)$ avec $d \geqslant 2$, la limite

$$
\lim _{\substack{t \rightarrow 0 \\ t>0}} \widetilde{\mathcal{A}}^{t}(\phi)\left(x^{\prime}\right)
$$

existe; on note cette limite $\widetilde{\mathcal{A}}(\phi)\left(x^{\prime}\right)$. De plus, on a

$$
\begin{aligned}
& \widetilde{\mathcal{A}}(\phi)\left(x^{\prime}\right) \\
= & \operatorname{sign}(\alpha) A_{\langle\phi\rangle}-B_{\langle\phi\rangle}+\pi^{2} \varepsilon\left(\phi\left(x_{1}^{\prime}, x_{2}^{\prime}\right)-\phi\left(x_{2}^{\prime}, x_{1}^{\prime}\right)\right) \\
& +\int_{\mathbb{R}^{2}}\left(\ln \left|x_{1}-x_{2}^{\prime}\right| \ln \left|x_{2}-x_{1}^{\prime}\right|-\ln \left|x_{1}-x_{1}^{\prime}\right| \ln \left|x_{2}-x_{2}^{\prime}\right|\right) \partial_{1,2} \phi(x) d x \\
& -\mathrm{i} \pi \varepsilon\left(\int_{\mathbb{R}} \ln \left|u-x_{2}^{\prime}\right| \partial_{1} \phi\left(u, x_{1}^{\prime}\right) d u+\int_{\mathbb{R}} \ln \left|u-x_{2}^{\prime}\right| \partial_{2} \phi\left(x_{1}^{\prime}, u\right) d u\right) \\
& -\mathrm{i} \pi\left(\int_{\mathbb{R}} \ln \left|u-x_{1}^{\prime}\right| \partial_{2} \phi\left(x_{2}^{\prime}, u\right) d u+\int_{\mathbb{R}} \ln \left|u-x_{1}^{\prime}\right| \partial_{1} \phi\left(u, x_{2}^{\prime}\right) d u\right) .
\end{aligned}
$$

Démonstration. - On obtient les relations

$$
\begin{aligned}
& \widetilde{\mathcal{A}}^{t}(\phi) \\
& =\int_{\mathbb{R}^{2}}\left\{-\frac{1}{\left(x_{1}+\mathrm{i} \varepsilon t-x_{1}^{\prime}\right)\left(x_{2}+\mathrm{i} t-x_{2}^{\prime}\right)}+\frac{1}{\left(x_{2}-\mathrm{i} \varepsilon t-x_{1}^{\prime}\right)\left(x_{1}-\mathrm{i} t-x_{2}^{\prime}\right)}\right\} \phi(x) d x \\
& =\int_{\mathbb{R}}\left(\frac{\ln \left(x_{2}+\mathrm{i} \varepsilon t-x_{1}^{\prime}\right)}{x_{2}+\mathrm{i} t-x_{2}^{\prime}}-\frac{\ln \left(x_{2}-\mathrm{i} t-x_{2}^{\prime}\right)}{x_{2}-\mathrm{i} \varepsilon t-x_{1}^{\prime}}\right)\langle\phi\rangle\left(x_{2}\right) d x_{2} \\
& +\int_{\mathbb{R}}\left(\ln \left(x_{1}+\mathrm{i} \varepsilon t-x_{1}^{\prime}\right) \ln \left(x_{1}+\mathrm{i} t-x_{2}^{\prime}\right)-\ln \left(x_{1}-\mathrm{i} t-x_{2}^{\prime}\right) \ln \left(x_{1}-\mathrm{i} \varepsilon t-x_{1}^{\prime}\right)\right) \\
& \left.+\int_{\mathbb{R}^{2}}\left(\ln \left(x_{1}-\mathrm{i} t-x_{2}^{\prime}\right) \ln \left(x_{2}-\mathrm{i} \varepsilon t-x_{1}^{\prime}\right) d x_{1}\right) \ln \left(x_{1}+\mathrm{i} \varepsilon t-x_{1}^{\prime}\right) \ln \left(x_{2}+\mathrm{i} t-x_{2}^{\prime}\right)\right) \\
& \partial_{1,2} \phi(x) d x .
\end{aligned}
$$

On sait d'après le lemme 3.3.7 que

$$
\lim _{\substack{t \rightarrow 0 \\ t>0}} F_{\langle\phi\rangle}^{t}\left(x_{1}^{\prime}, x_{2}^{\prime}, \varepsilon, 1\right)=F_{\langle\phi\rangle}\left(x_{1}^{\prime}, x_{2}^{\prime}, \varepsilon, 1\right)
$$

et

$$
\lim _{\substack{t \rightarrow 0 \\ t>0}} F_{\langle\phi\rangle}^{t}\left(x_{2}^{\prime}, x_{1}^{\prime},-1,-\varepsilon\right)=F_{\langle\phi\rangle}\left(x_{2}^{\prime}, x_{1}^{\prime},-1,-\varepsilon\right) .
$$


De plus, on a la majoration suivante pour $0<t<1$

$$
\left|\ln \left(x_{1}+\mathrm{i} \pm t-x_{1}^{\prime}\right)\right| \leqslant \frac{1}{2} \ln \left(\left(x_{1}-x_{1}^{\prime}\right)^{2}+1\right)+\pi
$$

où le terme de droite est une fonction localement intégrable en $x_{1}$. On en déduit que l'on peut prendre la limite en $t=0$ de chaque terme de l'expression (3.4.11), en utilisant le théorème de la convergence dominée pour les deux derniers termes; on obtient :

$$
\begin{aligned}
& F_{\langle\phi\rangle}\left(x_{1}^{\prime}, x_{2}^{\prime}, \varepsilon, 1\right)-F_{\langle\phi\rangle}\left(x_{2}^{\prime}, x_{1}^{\prime},-1,-\varepsilon\right) \\
& +2 \mathrm{i} \pi\left\{\int_{\mathbb{R}} \mu\left(v-x_{2}^{\prime}\right) \ln \left|v-x_{1}^{\prime}\right|\left\langle\partial_{1} \phi\right\rangle(v) d v+\varepsilon \int_{\mathbb{R}} \mu\left(v-x_{1}^{\prime}\right) \ln \left|v-x_{2}^{\prime}\right|\left\langle\partial_{1} \phi\right\rangle(v) d v\right\} \\
& +\int_{\mathbb{R}^{2}}\left(\ln \left|x_{1}-x_{2}^{\prime}\right| \ln \left|x_{2}-x_{1}^{\prime}\right|-\ln \left|x_{1}-x_{1}^{\prime}\right| \ln \left|x_{2}-x_{2}^{\prime}\right|\right) \partial_{1,2} \phi(x) d x \\
& -\pi^{2} \varepsilon \int_{\mathbb{R}^{2}}\left(\mu\left(x_{1}-x_{2}^{\prime}\right) \mu\left(x_{2}-x_{1}^{\prime}\right)-\mu\left(x_{1}-x_{1}^{\prime}\right) \mu\left(x_{2}-x_{2}^{\prime}\right)\right) \partial_{1,2} \phi(x) d x \\
& -\mathrm{i} \pi \varepsilon \int_{\mathbb{R}^{2}}\left(\ln \left|x_{1}-x_{2}^{\prime}\right| \mu\left(x_{2}-x_{1}^{\prime}\right)+\ln \left|x_{2}-x_{2}^{\prime}\right| \mu\left(x_{1}-x_{1}^{\prime}\right)\right) \partial_{1,2} \phi(x) d x \\
& -\mathrm{i} \pi \int_{\mathbb{R}^{2}}\left(\ln \left|x_{2}-x_{1}^{\prime}\right| \mu\left(x_{1}-x_{2}^{\prime}\right)+\ln \left|x_{1}-x_{1}^{\prime}\right| \mu\left(x_{2}-x_{2}^{\prime}\right)\right) \partial_{1,2} \phi(x) d x .
\end{aligned}
$$

Grâce au lemme 3.3.7, on a :

$$
\begin{aligned}
& F_{\langle\phi\rangle}\left(x_{1}^{\prime}, x_{2}^{\prime}, \varepsilon, 1\right)-F_{\langle\phi\rangle}\left(x_{2}^{\prime}, x_{1}^{\prime},-1,-\varepsilon\right) \\
& =\ln |2 \alpha|\left(H_{\langle\phi\rangle}^{\prime}\left(x_{1}^{\prime}\right)-H_{\langle\phi\rangle}^{\prime}\left(x_{2}^{\prime}\right)\right)-\operatorname{sign}(\alpha) \pi^{2} \varepsilon\langle\phi\rangle(\beta-|\alpha|) \\
& -\mathrm{i} \varepsilon \pi \int_{v \leqslant x_{1}^{\prime}} \ln \left|x_{2}^{\prime}-v\right|\langle\phi\rangle^{\prime}(v) d v-\mathrm{i} \pi \int_{v \leqslant x_{2}^{\prime}} \ln \left|x_{1}^{\prime}-v\right|\langle\phi\rangle^{\prime}(v) d v \\
& +\operatorname{sign}(\alpha) \int_{\mathbb{R}} \omega(u)\langle\phi\rangle(2 \alpha u+\alpha+\beta) d u+\operatorname{sign}(\alpha) \int_{\mathbb{R}} \omega(u)\langle\phi\rangle(-2 \alpha u-\alpha+\beta) d u \\
& =-\operatorname{sign}(\alpha) \pi^{2} \varepsilon\langle\phi\rangle(\beta-|\alpha|)+\operatorname{sign}(\alpha) A_{\langle\phi\rangle}-B_{\langle\phi\rangle} \\
& -\mathrm{i} \varepsilon \pi \int_{v \leqslant x_{1}^{\prime}} \ln \left|x_{2}^{\prime}-v\right|\langle\phi\rangle^{\prime}(v) d v-\mathrm{i} \pi \int_{v \leqslant x_{2}^{\prime}} \ln \left|x_{1}^{\prime}-v\right|\langle\phi\rangle^{\prime}(v) d v .
\end{aligned}
$$

Le lemme 3.4.7 ci-dessous et l'égalité (3.4.13) permettent de simplifier (3.4.12) ainsi

$$
\begin{aligned}
\operatorname{sign}(\alpha) & A_{\langle\phi\rangle}-B_{\langle\phi\rangle}+\pi^{2} \varepsilon\left(\phi\left(x_{1}^{\prime}, x_{2}^{\prime}\right)-\phi\left(x_{2}^{\prime}, x_{1}^{\prime}\right)\right) \\
& +\int_{\mathbb{R}^{2}}\left(\ln \left|x_{1}-x_{2}^{\prime}\right| \ln \left|x_{2}-x_{1}^{\prime}\right|-\ln \left|x_{1}-x_{1}^{\prime}\right| \ln \left|x_{2}-x_{2}^{\prime}\right|\right) \partial_{1,2} \phi(x) d x \\
& -\mathrm{i} \pi \varepsilon\left(\int_{\mathbb{R}} \ln \left|v-x_{2}^{\prime}\right| \partial_{1} \phi\left(v, x_{1}^{\prime}\right) d v+\int_{\mathbb{R}} \ln \left|v-x_{2}^{\prime}\right| \partial_{2} \phi\left(x_{1}^{\prime}, v\right) d v\right) \\
& -\mathrm{i} \pi\left(\int_{\mathbb{R}} \ln \left|v-x_{1}^{\prime}\right| \partial_{2} \phi\left(x_{2}^{\prime}, v\right) d v+\int_{\mathbb{R}} \ln \left|v-x_{1}^{\prime}\right| \partial_{1} \phi\left(v, x_{2}^{\prime}\right) d v\right) .
\end{aligned}
$$


Le lemme suivant est utilisé dans la démonstration du lemme précédent.

LEMME 3.4.7. - On a les relations suivantes :

$$
\begin{gathered}
\int_{\mathbb{R}^{2}}\left(\mu\left(x_{1}-x_{2}^{\prime}\right) \mu\left(x_{2}-x_{1}^{\prime}\right)-\mu\left(x_{1}-x_{1}^{\prime}\right) \mu\left(x_{2}-x_{2}^{\prime}\right)\right) \partial_{1,2} \phi(x) d x \\
=\phi\left(x_{2}^{\prime}, x_{1}^{\prime}\right)-\phi\left(x_{1}^{\prime}, x_{2}^{\prime}\right)-\operatorname{sign}(\alpha)\langle\phi\rangle(\beta-|\alpha|) \\
\int_{\mathbb{R}^{2}}\left(\ln \left|x_{1}-x_{1}^{\prime}\right| \mu\left(x_{2}-x_{2}^{\prime}\right)+\ln \left|x_{2}-x_{1}^{\prime}\right| \mu\left(x_{1}-x_{2}^{\prime}\right)\right) \partial_{1,2} \phi d x \\
=\int_{\mathbb{R}} \ln \left|v-x_{1}^{\prime}\right| \partial_{2} \phi\left(x_{2}^{\prime}, v\right) d v+\int_{\mathbb{R}} \ln \left|v-x_{1}^{\prime}\right| \partial_{1} \phi\left(v, x_{2}^{\prime}\right) d v .
\end{gathered}
$$

Démonstration. - En intégrant par parties deux fois, on a :

$$
\begin{aligned}
\int_{\mathbb{R}^{2}} \mu\left(x_{1}-x_{2}^{\prime}\right) \mu & \left(x_{2}-x_{1}^{\prime}\right) \partial_{1,2} \phi(x) d x \\
& =\phi\left(x_{2}^{\prime}, x_{1}^{\prime}\right)+\mu\left(x_{2}^{\prime}-x_{1}^{\prime}\right)\langle\phi\rangle\left(x_{2}^{\prime}\right)-\int_{x_{2} \leqslant \min \left(x_{1}^{\prime}, x_{2}^{\prime}\right)}\left\langle\partial_{2} \phi\right\rangle\left(x_{2}\right) d x_{2} .
\end{aligned}
$$

En échangeant les rôles de $x_{1}^{\prime}$ et $x_{2}^{\prime}$, on a l'égalité

$$
\begin{aligned}
\int_{\mathbb{R}^{2}} \mu\left(x_{1}-x_{1}^{\prime}\right) \mu & \left(x_{2}-x_{2}^{\prime}\right) \partial_{1,2} \phi(x) d x \\
& =\phi\left(x_{1}^{\prime}, x_{2}^{\prime}\right)+\mu\left(x_{1}^{\prime}-x_{2}^{\prime}\right)\langle\phi\rangle\left(x_{1}^{\prime}\right)-\int_{x_{2} \leqslant \min \left(x_{1}^{\prime}, x_{2}^{\prime}\right)}\left\langle\partial_{2} \phi\right\rangle\left(x_{2}\right) d x_{2} .
\end{aligned}
$$

La différence des deux expressions précédentes permet d'obtenir la première relation.

En intégrant par partie, on a :

$$
\begin{aligned}
\int_{\mathbb{R}^{2}} \ln \left|x_{1}-x_{1}^{\prime}\right| \mu\left(x_{2}-\right. & \left.x_{2}^{\prime}\right) \partial_{1,2} \phi(x) d x \\
& =\int_{\mathbb{R}} \ln \left|v-x_{1}^{\prime}\right| \partial_{1} \phi\left(v, x_{2}^{\prime}\right) d v+\int_{v \leqslant x_{2}^{\prime}} \ln \left|v-x_{1}^{\prime}\right|\left\langle\partial_{1} \phi\right\rangle(v) d v .
\end{aligned}
$$

De même, on a :

$$
\begin{aligned}
\int_{\mathbb{R}^{2}} \ln \left|x_{2}-x_{1}^{\prime}\right| \mu\left(x_{1}-\right. & \left.x_{2}^{\prime}\right) \partial_{1,2} \phi(x) d x \\
& =\int_{\mathbb{R}} \ln \left|v-x_{1}^{\prime}\right| \partial_{2} \phi\left(x_{2}^{\prime}, v\right) d v-\int_{v \leqslant x_{2}^{\prime}} \ln \left|v-x_{1}^{\prime}\right|\left\langle\partial_{2} \phi\right\rangle(v) d v .
\end{aligned}
$$

Comme $\left\langle\left(\partial_{1}-\partial_{2}\right) \phi\right\rangle=0$, en sommant les deux expressions précédentes, on obtient l'égalité recherchée.

Les quatre lemmes suivants permettent de compléter les propriétés de l'expression de $\widetilde{\mathcal{A}}(\phi)$ en étudiant les expressions (3.4.8), (3.4.9) et (3.4.10). On introduit les notations suivantes : 
Pour $\phi \in \mathcal{W}_{c}\left(\mathfrak{h}_{\varnothing}, d\right)$, on désigne par $\phi_{\geqslant}\left(\right.$resp. $\left.\phi_{\leqslant}\right)$le prolongement par continuité sur le fermé $\left\{x_{1} \geqslant x_{2}\right\}$ (resp. $\left\{x_{1} \leqslant x_{2}\right\}$ ) de $\phi$.

Lemme 3.4.8. - Pour $\phi \in \mathcal{W}_{c}\left(\mathfrak{h}_{\varnothing}, d\right)$ avec $d \geqslant 1$, on a $\left(\partial_{2}+\partial_{1}\right) \phi \in \mathcal{W}_{c}\left(\mathfrak{h}_{\varnothing}, d-1\right)$.

Démonstration. - La fonction $\left(\partial_{2}+\partial_{1}\right) \phi$ est de classe $C^{d-1}$ sur $\mathfrak{h}_{\varnothing}^{\text {reg }}$ et pour $w \in$ $\mathcal{E}\left(\mathfrak{h}_{\varnothing}, d-1\right)$, la fonction $\partial(w) \partial(\beta) \phi$ se prolonge par continuité sur l'adhérence de chaque composante connexe de $\mathfrak{h}_{\varnothing}^{\text {reg }}$. Puis, on a pour $p, q \in \mathbb{N}$ avec $p$ impair

$$
\left\langle\left(\left(\partial_{2}-\partial_{1}\right)^{p}\left(\partial_{2}+\partial_{1}\right)^{q+1}\right) \phi\right\rangle=0 .
$$

Lemme 3.4.9. - Pour $\phi \in \mathcal{W}_{c}\left(\mathfrak{h}_{\varnothing}, d\right)$, il existe $\theta_{\geqslant}$et $\theta_{\leqslant}$dans $\mathcal{C}_{c}^{d}\left(\mathbb{R}^{2}\right)$ qui prolongent respectivement $\phi_{\geqslant}$et $\phi_{\leqslant}$.

Démonstration. - Montrons le résultat pour $\phi_{\geqslant}$, la démonstration est identique pour $\phi_{\leqslant}$. Pour montrer ce résultat, on utilise le théorème de prolongement de Whitney. Pour $(m, n) \in \mathbb{N}^{2}$ tel que $m+n \leqslant d$, on note $\phi_{\geqslant}^{m, n}$ le prolongement par continuité de la fonction $\partial_{1}^{m} \partial_{2}^{n} \psi$ définie sur l'ouvert $\left\{x \in \mathbb{R}^{2} \mid x_{1}>x_{2}\right\}$ à $\left\{x \in \mathbb{R}^{2} \mid x_{1} \geqslant x_{2}\right\}$. Soit $\mathcal{K}$ une partie compacte convexe de $\left\{x \in \mathbb{R}^{2} \mid x_{1} \geqslant x_{2}\right\}$ telle que $\operatorname{Supp}\left(\phi_{\geqslant}\right) \subset \mathcal{K}, \mathcal{K}^{\prime}$ une partie compacte et $\chi \in \mathcal{D}\left(\mathbb{R}^{2}\right)$ telle que $\chi(x)=1$ pour $x \in \operatorname{Supp}\left(\phi_{\geqslant}\right)$. La famille de fonctions $\left(\phi_{\geqslant}^{m, n}\right)_{m+n \leqslant d}$ constitue un jet d'ordre $d$ sur $\mathcal{K}$ (cf. [9, p.68]). D'après la définition de $\phi_{\geqslant}^{m, n}$, on a pour $m, n, r \in \mathbb{N}$ tels que $m+n \leqslant r$ et $x, y \in \mathcal{K}$

$$
\phi_{\geqslant}^{m, n}(y)-\sum_{p+q \leqslant r-m-n} \frac{\left(y_{1}-x_{1}\right)^{p}\left(y_{2}-x_{2}\right)^{q}}{p ! q !} \phi_{\geqslant}^{m+p, n+q}(x)=o\left(|y-x|^{r-m-n}\right)
$$

ainsi, $\left(\phi_{\geqslant}^{m, n}\right)_{m+n \leqslant d}$ est une fonction de Whitney de classe $C^{d}$ (cf. [9, Définition 1.6]) sur $\mathcal{K}$. Il existe donc d'après le Théorème 2.2 de $[\mathbf{9}]$, une fonction $f$ de classe $C^{d}$ sur $\mathbb{R}^{2}$ qui prolonge $\phi_{\geqslant} \mid \mathcal{K}$, la fonction $f \chi$ est de classe $C^{d}$ sur $\mathbb{R}^{2}$ et prolonge $\phi_{\geqslant}$en effet, on a $\phi_{\geqslant} \chi=\phi_{\geqslant}$ainsi, la fonction $\theta_{\geqslant}=f \chi$ convient.

LEmme 3.4.10. - Soit $\phi \in \mathcal{W}_{c}\left(\mathfrak{h}_{\varnothing}, d+1\right)$. Les fonctions suivantes appartiennent $\grave{a}$ $\mathcal{C}^{d}\left(\mathbb{R}^{2}\right)$ :

$$
\begin{gathered}
\int_{\mathbb{R}} \ln \left|u-x_{1}^{\prime}\right| \partial_{2} \phi\left(x_{2}^{\prime}, u\right) d u+\int_{\mathbb{R}} \ln \left|u-x_{1}^{\prime}\right| \partial_{1} \phi\left(u, x_{2}^{\prime}\right) d u . \\
\int_{\mathbb{R}} \ln \left|u-x_{2}^{\prime}\right| \partial_{1} \phi\left(u, x_{1}^{\prime}\right) d u+\int_{\mathbb{R}} \ln \left|u-x_{2}^{\prime}\right| \partial_{2} \phi\left(x_{1}^{\prime}, u\right) d u .
\end{gathered}
$$

Démonstration. - On étudie tout d'abord la fonction (3.4.14); pour $\phi \in$ $\mathcal{W}_{c}\left(\mathfrak{h}_{\varnothing}, d+1\right)$, on a l'égalité suivante :

$$
\begin{aligned}
\int_{\mathbb{R}} \ln \left|u-x_{1}^{\prime}\right| \partial_{2} \psi & \left(x_{2}^{\prime}, u\right) d u+\int_{\mathbb{R}} \ln \left|u-x_{1}^{\prime}\right| \partial_{1} \psi\left(u, x_{2}^{\prime}\right) d u \\
& =\int_{\mathbb{R}} \ln |u|\left(\partial_{2} \psi(\beta+\alpha, u+\beta-\alpha)+\partial_{1} \psi(\beta-\alpha+u, \beta+\alpha)\right) d u .
\end{aligned}
$$


On note $E_{\phi}(\alpha, \beta)$ l'expression précédente, montrons que cette fonction est de classe $C^{d}$ sur l'ouvert $\{\alpha \neq 0\} \subset \mathbb{R}^{2}$. Il existe une fonction de $\mathcal{C}_{c}^{d+1}\left(\mathbb{R}^{2}\right), \theta_{\geqslant}$(resp. $\left.\theta_{\leqslant}\right)$qui prolonge $\phi_{\geqslant}$(resp. $\phi_{\leqslant}$) d'après le lemme 3.4.9. Pour $x^{\prime} \in \mathfrak{h}^{\text {'reg }}$, on a l'égalité

$$
\begin{aligned}
& \int_{\mathbb{R}} \ln |u| \partial_{2} \phi(\beta+\alpha, u+\beta-\alpha) d u \\
& =\int_{u \geqslant 2 \alpha} \ln |u| \partial_{2} \theta_{\leqslant}(\beta+\alpha, u+\beta-\alpha) d u+\int_{u \leqslant 2 \alpha} \ln |u| \partial_{2} \theta_{\geqslant}(\beta+\alpha, u+\beta-\alpha) d u .
\end{aligned}
$$

Les fonctions

$$
\begin{aligned}
& Z_{\geqslant}:\left(\tau, x^{\prime}\right) \in \mathbb{R} \backslash\{0\} \times \mathfrak{h}_{\varnothing}^{\prime} \longmapsto \int_{u \geqslant \tau} \ln |u| \partial_{2} \theta_{\leqslant}(\beta+\alpha, u+\beta-\alpha) d u \\
& Z_{\leqslant}:\left(\tau, x^{\prime}\right) \in \mathbb{R} \backslash\{0\} \times \mathfrak{h}_{\varnothing}^{\prime} \longmapsto \int_{u \leqslant \tau} \ln |u| \partial_{2} \theta_{\geqslant}(\beta+\alpha, u+\beta-\alpha) d u
\end{aligned}
$$

sont de classe $C^{d}$. De plus, on a pour $a, b \in \mathbb{N}$ tels que $a+b \leqslant d$

$$
\partial\left(\alpha^{a} \beta^{b}\right) Z_{\geqslant}\left(\tau, x^{\prime}\right)=\int_{u \geqslant \tau} \ln |u|\left(\partial_{1}-\partial_{2}\right)^{a}\left(\partial_{1}+\partial_{2}\right)^{b} \partial_{2} \theta_{\leqslant}(\beta+\alpha, u+\beta-\alpha) d u
$$

et

$$
\partial\left(\tau \alpha^{a} \beta^{b}\right) Z_{\geqslant}\left(\tau, x^{\prime}\right)=-\ln |\tau|\left(\partial_{1}-\partial_{2}\right)^{a}\left(\partial_{1}+\partial_{2}\right)^{b} \partial_{2} \theta_{\leqslant}(\beta+\alpha, \tau+\beta-\alpha) d u
$$

Comme

$$
\int_{\mathbb{R}} \ln |u| \partial_{2} \phi(\beta+\alpha, u+\beta-\alpha) d u=Z_{\geqslant}(2 \alpha, \alpha, \beta)+Z_{\leqslant}(2 \alpha, \alpha, \beta)
$$

on en déduit que $E_{\phi}$ est une fonction de classe $C^{d}$ sur l'ouvert $\{\alpha \neq 0\} \subset \mathbb{R}^{2}$. On observe ensuite que d'après (3.4.16) et (3.4.17), on a

$$
\partial_{\beta} E_{\phi}(\alpha, \beta)=E_{\partial_{1} \phi+\partial_{2} \phi}(\alpha, \beta) .
$$

On en déduit qu'il reste à démontrer que $\partial_{\alpha}^{n} E_{\phi}$ se prolonge en une fonction continue sur $\mathbb{R}^{2}$ pour $\phi \in \mathcal{W}_{c}\left(\mathfrak{h}_{\varnothing}, d+1\right)$ avec $n \leqslant d$ pour obtenir le résultat. Calculons cette dérivée par récurrence sur $n$. Supposons que l'on a :

$$
\begin{aligned}
\partial_{\alpha}^{n} E_{\phi}(\alpha, \beta)=\sum_{p=0}^{n}( & -1)^{n-p} \mathcal{C}_{n}^{p} \int_{\mathbb{R}} \partial_{1,2}^{p, n-p+1} \phi(\beta+\alpha, \beta+u-\alpha) \ln |u| d u \\
& +\sum_{p=0}^{n}(-1)^{p} \mathcal{C}_{n}^{p} \int_{\mathbb{R}} \partial_{1,2}^{p+1, n-p} \phi(\beta-\alpha+u, \beta+\alpha) \ln |u| d u
\end{aligned}
$$


On a alors en dérivant de nouveau en $\alpha$ :

$$
\begin{gathered}
\partial_{\alpha}^{n+1} E_{\phi}(\alpha, \beta)=\sum_{p=0}^{n+1}(-1)^{n+1-p} \mathcal{C}_{n+1}^{p} \int_{\mathbb{R}} \partial_{1,2}^{p, n+1-p+1} \phi(\beta+\alpha, \beta+u-\alpha) \ln |u| d u \\
+\sum_{p=0}^{n+1}(-1)^{p} \mathcal{C}_{n+1}^{p} \int_{\mathbb{R}} \partial_{1,2}^{p+1, n+1-p} \phi(\beta-\alpha+u, \beta+\alpha) \ln |u| d u \\
+\ln |2 \alpha| \sum_{p=0}^{n}(-1)^{n-p} \mathcal{C}_{n}^{p}\left\langle\partial_{1,2}^{p, n-p+1} \phi\right\rangle(\beta+\alpha) \\
-\ln |2 \alpha| \sum_{p=0}^{n}(-1)^{p} \mathcal{C}_{n}^{p}\left\langle\partial_{1,2}^{p+1, n-p} \phi\right\rangle(\beta+\alpha) .
\end{gathered}
$$

On considère l'opérateur différentiel $D$ suivant

$$
D=\sum_{p=0}^{n}(-1)^{n-p} \mathcal{C}_{n}^{p} \partial_{1,2}^{p, n-p+1}-\sum_{p=0}^{n}(-1)^{p} \mathcal{C}_{n}^{p} \partial_{1,2}^{p+1, n-p}
$$

Les deux derniers termes de l'expression (3.4.19) s'écrivent :

$$
\ln |2 \alpha|\langle D \phi\rangle(\beta+\alpha)
$$

On observe que

$$
D=\left(\partial_{1}-\partial_{2}\right)^{n} \partial_{2}-\left(\partial_{2}-\partial_{1}\right)^{n} \partial_{1}
$$

ainsi

$$
D= \begin{cases}-\left(\partial_{2}-\partial_{1}\right)^{n}\left(\partial_{2}+\partial_{1}\right) & \text { si } n \text { est impair } \\ \left(\partial_{2}-\partial_{1}\right)^{n+1} & \text { si } n \text { est pair. }\end{cases}
$$

On en déduit

$$
\ln |2 \alpha|\langle D \phi\rangle(\beta+\alpha)=0,
$$

et l'égalité (3.4.18) est démontrée par récurrence, ainsi $E_{\phi}(\alpha, \beta)$ est de classe $C^{d}$ sur $\mathbb{R}^{2}$ pour $\phi \in \mathcal{W}_{c}\left(\mathfrak{h}_{\varnothing}, d+1\right)$. La démonstration est identique pour (3.4.15).

DÉfinition 3.4.11. - Pour $\phi \in \mathcal{W}_{c}\left(\mathfrak{h}_{\varnothing}, d\right)$ avec $d \geqslant 2$, on note $G_{\phi}$ la fonction définie sur $\mathbb{R}^{2}$ par

$$
\int_{\mathbb{R}^{2}}\left(\ln \left|x_{1}-\beta-\alpha\right| \ln \left|x_{2}-\beta+\alpha\right|-\ln \left|x_{1}-\beta+\alpha\right| \ln \left|x_{2}-\beta-\alpha\right|\right) \partial_{1,2} \phi(x) d x .
$$

Le lemme suivant donne les propriétés de l'expression (3.4.8).

Lemme 3.4.12. - Soit $\phi \in \mathcal{W}_{c}\left(\mathfrak{h}_{\varnothing}, d+3\right)$. On a les propriétés suivantes pour :

(1) $G_{\phi}(\alpha, \beta)$ appartient à $\mathcal{H}(\{\alpha \neq 0\}, d)$.

(2) $\partial_{\beta} G_{\phi}=G_{\left(\partial_{1}+\partial_{2}\right) \phi}$. 
(3) $\partial_{\alpha}^{n} G_{\phi}=\left\{\begin{array}{cc}\text { une fonction continue } & \text { si } n \text { est impair, } \\ -\operatorname{sign}(\alpha) \pi^{2}\left(\langle\phi\rangle^{(n)}(\beta)-\left\langle\left(\partial_{2}-\partial_{1}\right)^{(n)} \phi\right\rangle(\beta)\right) & \\ \text { modulo une fonction continue sur } \mathbb{R}^{2} & \text { si } n \text { est pair, }\end{array}\right.$ pour $n \leqslant d$.

Démonstration. - On pose pour $\phi \in \mathcal{H}_{c}\left(\mathfrak{h}_{\varnothing}, d+3\right)$,

$$
L_{\phi}(\alpha, \beta)=\int_{\mathbb{R}^{2}} \ln \left|x_{1}\right| \ln \left|x_{2}\right| \partial_{1,2} \phi\left(x_{1}+\beta+\alpha, x_{2}+\beta-\alpha\right) d x_{1} d x_{2},
$$

on a ainsi la relation

$$
G_{\phi}(\alpha, \beta)=L_{\phi}(\alpha, \beta)-L_{\phi}(-\alpha, \beta)
$$

pour $\phi \in \mathcal{W}_{c}\left(\mathfrak{h}_{\varnothing}, d+3\right)$. Montrons que $L_{\phi}$ est de classe $C^{d}$ sur l'ouvert $\{\alpha \neq 0\}$ pour $\phi \in \mathcal{H}_{c}\left(\mathfrak{h}_{\varnothing}, d+3\right)$. On note $\theta_{\leqslant}\left(\right.$resp. $\left.\theta_{\geqslant}\right)$un prolongement de $\phi_{\leqslant}\left(\right.$resp. $\left.\phi_{\geqslant}\right)$à $\mathbb{R}^{2}$; la démonstration du lemme 3.4.9 s'applique aussi pour $\phi \in \mathcal{H}_{c}\left(\mathfrak{h}_{\varnothing}, d+3\right)$. On obtient la relation

$$
\begin{aligned}
L_{\phi}(\alpha, \beta)= & \int_{\left\{x_{2}-x_{1} \geqslant 2 \alpha\right\}} \ln \left|x_{1}\right| \ln \left|x_{2}\right| \partial_{1,2} \theta_{\leqslant}\left(x_{1}+\beta+\alpha, x_{2}+\beta-\alpha\right) d x_{1} d x_{2} \\
& \quad+\int_{\left\{x_{2}-x_{1} \leqslant 2 \alpha\right\}} \ln \left|x_{1}\right| \ln \left|x_{2}\right| \partial_{1,2} \theta_{\geqslant}\left(x_{1}+\beta+\alpha, x_{2}+\beta-\alpha\right) d x_{1} d x_{2}
\end{aligned}
$$

En posant $s=\left(x_{1}+x_{2}\right) / 2$ et $r=\left(x_{2}-x_{1}\right) / 2$, l'égalité précédente devient

$$
\begin{aligned}
L_{\phi}(\alpha, \beta)= & 2 \int_{\{r \geqslant \alpha\}} \ln |s-r| \ln |s+r| \partial_{1,2} \theta_{\leqslant}(s-r+\beta+\alpha, s+r+\beta-\alpha) d s d r \\
& +2 \int_{\{r \leqslant \alpha\}} \ln |s-r| \ln |s+r| \partial_{1,2} \theta_{\geqslant}(s-r+\beta+\alpha, s+r+\beta-\alpha) d s d r
\end{aligned}
$$

On en déduit que $L_{\phi}$ est dérivable en $\beta$ et en $\alpha$ (à l'ordre 1 ); on obtient les relations suivantes

$$
\begin{gathered}
\partial_{\beta} L_{\phi}(\alpha, \beta)=L_{\left(\partial_{1}+\partial_{2}\right) \phi}(\alpha, \beta) \\
\partial_{\alpha} L_{\phi}(\alpha, \beta)=L_{\left(\partial_{1}-\partial_{2}\right)(\phi)}(\alpha, \beta)+2 C_{\left\langle\partial_{1,2} \phi\right\rangle}(\alpha, \beta) .
\end{gathered}
$$

À l'ordre $n \geqslant 1$, on obtient par récurrence pour $\alpha \neq 0$ :

$$
\partial_{\alpha}^{n} L_{\phi}(\alpha, \beta)=L_{\left(\partial_{1}-\partial_{2}\right)^{n}(\phi)}(\alpha, \beta)+2 \sum_{p=0}^{n-1}\left(\partial_{\alpha}^{n-p-1} C_{\left\langle\left(\partial_{1}-\partial_{2}\right)^{p} \partial_{1,2} \phi\right\rangle}(\alpha, \beta)\right) .
$$

On considère maintenant $\phi \in \mathcal{W}_{c}\left(\mathfrak{h}_{\varnothing}, d+3\right)$, on obtient alors à partir de l'égalité précédente et de la relation

$$
G_{\phi}(\alpha, \beta)=L_{\phi}(\alpha, \beta)-L_{\phi}(-\alpha, \beta),
$$


la formule :

$$
\begin{aligned}
& \partial_{\alpha}^{n} G_{\phi}(\alpha, \beta)=L_{\left(\partial_{1}-\partial_{2}\right)^{n} \phi}(\alpha, \beta)-(-1)^{n} L_{\left(\partial_{2}-\partial_{1}\right)^{n} \phi}(-\alpha, \beta) \\
& +2 \sum_{p=0}^{n-1}\left(\partial_{\alpha}^{n-p-1} C_{\left\langle\left(\partial_{1}-\partial_{2}\right)^{p} \partial_{1,2} \phi\right\rangle}(\alpha, \beta)-(-1)^{n} \partial_{\alpha}^{n-p-1} C_{\left\langle\left(\partial_{1}-\partial_{2}\right)^{p} \partial_{1,2} \phi\right\rangle}(-\alpha, \beta)\right) .
\end{aligned}
$$

Comme

$$
\left\langle\left(\partial_{1}-\partial_{2}\right)^{p} \partial_{1,2} \phi\right\rangle=0
$$

pour $p$ est impair $\left(\phi \in \mathcal{W}_{c}\left(\mathfrak{h}_{\varnothing}, d+3\right)\right)$, l'expression (3.4.20) se simplifie ainsi

$$
+2 \sum_{\substack{p=0 \\ p \text { pair }}}^{n-1}\left(\partial_{\alpha}^{n-p-1} C_{\left\langle\left(\partial_{1}-\partial_{2}\right)^{p} \partial_{1,2} \phi\right\rangle}(\alpha, \beta)-(-1)^{n} \partial_{\alpha}^{n-p-1} C_{\left\langle\left(\partial_{1}-\partial_{2}\right)^{p} \partial_{1,2} \phi\right\rangle}(-\alpha, \beta)\right) .
$$

Si $n$ est impair, alors pour $p$ pair, d'après le lemme 3.3.8,

$$
\lim _{\alpha \rightarrow 0} \partial_{\alpha}^{n-p-1} C_{\left\langle\left(\partial_{1}-\partial_{2}\right)^{p} \partial_{1,2} \phi\right\rangle}(\alpha, \beta)
$$

existe, on en déduit la première partie du point (3). Si $n$ est pair, on a pour $p$ pair, d'après le lemme 3.3.8,

$$
\lim _{\substack{\alpha \rightarrow 0 \\ \operatorname{sign}(\alpha)=\varepsilon}} \partial_{\alpha}^{n-p-1} C_{\left\langle\left(\partial_{1}-\partial_{2}\right)^{p} \partial_{1,2} \phi\right\rangle}(\alpha, \beta)=-\varepsilon \pi^{2}\left\langle\left(\partial_{1}-\partial_{2}\right)^{p} \partial_{1,2} \phi\right\rangle{ }^{(n-p-2)}(\beta),
$$

de plus, on a les égalités suivantes

$$
\begin{aligned}
4 \sum_{\substack{p=0 \\
p \text { pair }}}^{n-1}\left\langle\left(\partial_{1}-\partial_{2}\right)^{p} \partial_{1,2} \phi\right\rangle^{(n-p-2)}(\beta) & \\
= & \sum_{\substack{p=0 \\
p \text { pair }}}^{n-1}\left(\left\langle\left(\partial_{1}-\partial_{2}\right)^{p} \phi\right\rangle^{(n-p)}-\left\langle\left(\partial_{1}-\partial_{2}\right)^{p+2} \phi\right\rangle^{(n-p-2)}\right) \\
= & \langle\phi\rangle^{(n)}(\beta)-\left\langle\left(\partial_{2}-\partial_{1}\right)^{(n)} \phi\right\rangle(\beta) ;
\end{aligned}
$$

on en déduit que l'expression (3.4.21) tend vers

$$
-\varepsilon \pi^{2}\left(\langle\phi\rangle^{(n)}-\left\langle\left(\partial_{2}-\partial_{1}\right)^{n} \phi\right\rangle(\beta)\right) .
$$

Le deuxième partie du point (3) est obtenue.

On identifie $\mathfrak{h}_{\varnothing}$ et $\mathfrak{h}_{\varnothing}^{\prime}$ en posant $x_{j}=x_{j}^{\prime}$ pour $j \in\{1,2\}$. On obtient la proposition suivante qui fournit les propriétés de $\widetilde{\mathcal{A}}$.

Proposition 3.4.13. - Soit $\phi \in \mathcal{W}_{c}\left(\mathfrak{h}_{\varnothing}, d+3\right)$, on a les propriétés suivantes :

(1) La fonction $\widetilde{\mathcal{A}}(\phi)$ appartient à $\mathcal{H}\left(\mathfrak{h}_{\varnothing}^{\text {'reg }}, d\right)$. Cela permet de définir la fonction saut $\langle\partial(w) \widetilde{\mathcal{A}}(\phi)\rangle$. 
(2) On a

(3) Pour $n \leqslant d$, on a l'égalité

$$
\partial_{\beta} \widetilde{\mathcal{A}}(\phi)=\widetilde{\mathcal{A}}\left(\left(\partial_{1}+\partial_{2}\right) \phi\right) .
$$

$$
\left\langle\partial_{\alpha}^{n} \widetilde{\mathcal{A}}(\phi)\right\rangle=-4 \pi^{2}\langle\phi\rangle^{(n)}(\beta)+2 \pi^{2}(1+\varepsilon)\left\langle\left(\partial_{2}-\partial_{1}\right)^{n} \phi\right\rangle(\beta)
$$

si $n$ est pair et 0 sinon.

Démonstration. - D'après le lemme 3.3.12, l'expression (3.4.7) appartient à $\mathcal{H}\left(\mathfrak{h}_{\varnothing}^{\text {'reg }}, d\right)$. Il en est de même pour (3.4.8) d'après le lemme 3.4.12. Et enfin, les expressions (3.4.9) et (3.4.10) sont des fonctions de classe $C^{d}$ sur $\mathfrak{h}_{\varnothing}^{\prime}$ d'après le lemme 3.4.10. On en déduit les points (1) et (2). D'après les lemmes 3.3 .12 et 3.4.12, les expressions (3.4.7) et (3.4.8) vérifient la propriété d'invariance par $\partial_{1}+\partial_{2}$, comme celle-ci est clair pour les expressions (3.4.9) et (3.4.10), on en déduit que

$$
\partial_{\beta} \widetilde{\mathcal{A}}(\phi)=\widetilde{\mathcal{A}}\left(\left(\partial_{1}+\partial_{2}\right) \phi\right)
$$

et le point (3) est vérifié. Pour $n \leqslant d$ impair, la dérivée d'ordre $n$ en $\alpha$ de l'expression (3.4.7) est égale à

$$
\operatorname{sign}(\alpha) \partial_{\alpha}^{n} A_{\langle\phi\rangle}-\partial_{\alpha}^{n} B_{\langle\phi\rangle}+\pi^{2} \varepsilon\left(\left(\partial_{2}-\partial_{1}\right)^{n} \phi\left(x_{1}^{\prime}, x_{2}^{\prime}\right)+\left(\partial_{2}-\partial_{1}\right)^{n} \phi\left(x_{2}^{\prime}, x_{1}^{\prime}\right)\right)
$$

Cette expression est continue d'après le lemme 3.3.12. De même, la dérivée d'ordre $n$ en $\alpha$ de l'expression (3.4.8) est une fonction continue d'après le lemme 3.4.12 ainsi, on obtient la deuxième partie du point (4) avec le lemme 3.4.10. Pour $n$ pair, la dérivée d'ordre $n$ en $\alpha$ de l'expression (3.4.7) est égale à

$$
\operatorname{sign}(\alpha) \partial_{\alpha}^{n} A_{\langle\phi\rangle}-\partial_{\alpha}^{n} B_{\langle\phi\rangle}+\pi^{2} \varepsilon\left(\left(\partial_{2}-\partial_{1}\right)^{n} \phi\left(x_{1}^{\prime}, x_{2}^{\prime}\right)-\left(\partial_{2}-\partial_{1}\right)^{n} \phi\left(x_{2}^{\prime}, x_{1}^{\prime}\right)\right) .
$$

On obtient le saut de cette expression

$$
-2 \pi^{2}\langle\phi\rangle^{(n)}(\beta)+2 \pi^{2} \varepsilon\left\langle\left(\partial_{2}-\partial_{1}\right)^{n} \phi\right\rangle(\beta)
$$

grâce au lemme 3.3.12. D'après le lemme 3.4.12, le saut de l'expression (3.4.8) est égal à

$$
-2 \pi^{2}\langle\phi\rangle^{(n)}(\beta)+2 \pi^{2}\left\langle\left(\partial_{2}-\partial_{1}\right)^{n} \phi\right\rangle(\beta)
$$

En sommant les deux dernières expressions, on obtient le résultat.

Le corollaire 3.4.5 et la proposition 3.4.13 permettent d'obtenir le résultat suivant.

Proposition 3.4.14. - Soient $\phi \in \mathcal{W}_{c}\left(\mathfrak{h}_{\varnothing}, d+3\right)$ et $\psi \in \mathcal{W}_{c}\left(\mathfrak{h}_{\mathcal{S}}, d+3\right)$ telles que

$$
\langle\partial(w) \phi\rangle=\mathrm{i} \partial\left(c_{\mathcal{S}}(w)\right) \psi \text {. }
$$

On a les propriétés suivantes :

(1) La fonction $\widetilde{\mathcal{A}}(\phi)+\widetilde{\mathcal{B}}(\psi)$ appartient à $\mathcal{H}\left(\mathfrak{h}_{\varnothing}^{\prime \text { reg }}, d\right)$.

(2) On a

$$
\partial_{\beta}(\widetilde{\mathcal{A}}(\phi)+\widetilde{\mathcal{B}}(\psi))=\widetilde{\mathcal{A}}\left(\left(\partial_{1}+\partial_{2}\right) \phi\right)+\widetilde{\mathcal{B}}\left(\left(\partial_{1}+\partial_{2}\right) \psi\right)
$$


(3) Pour $n \leqslant d$, on a l'égalité

$$
\left\langle\partial_{\alpha}^{n}(\widetilde{\mathcal{A}}(\phi)+\widetilde{\mathcal{B}}(\psi))\right\rangle(\beta)=2 \pi^{2}(1+\varepsilon)\left\langle\left(\partial_{2}-\partial_{1}\right)^{n} \phi\right\rangle(\beta) .
$$

On note $\partial_{z}$ l'opérateur différentiel de $\mathfrak{g}$ associé à l'élément $\left(\begin{array}{ll}\mathrm{i} & 0 \\ 0 & \mathrm{i}\end{array}\right)$ de $\operatorname{Sym}\left(\mathfrak{g}_{\mathbb{C}}\right)$.

Proposition 3.4.15. - Soit $\psi \in \mathcal{D}(\mathfrak{g})$, on a les propriétés suivantes :

(1) La fonction $\mathbf{C h c}(\psi)_{\varnothing}$ appartient à $\mathcal{H}\left(\mathfrak{h}_{\varnothing}^{\text {'reg }}\right)$.

(2) On a la relation

$$
\partial_{\beta} \operatorname{Chc}(\psi)_{\varnothing}=\operatorname{Chc}\left(\partial_{z} \psi\right)_{\varnothing}
$$

(3) Pour $n \in \mathbb{N}$, on a

$$
\left\langle\partial_{\alpha}^{n} \mathbf{C h c}(\psi)_{\varnothing}\right\rangle=32(\varepsilon+1) \pi^{2}\left\langle\partial_{\alpha}^{n} \psi_{\varnothing}\right\rangle(\beta) .
$$

Démonstration. - Cette proposition découle de la proposition 3.4.14; en effet, on a

$$
\operatorname{Chc}(\psi)_{\varnothing}\left(x^{\prime}\right)=C_{\mathfrak{g}, \mathfrak{g}^{\prime}}\left\{\widetilde{\mathcal{A}}\left(\psi_{\varnothing}\right)\left(x^{\prime}\right)+\widetilde{\mathcal{B}}\left(\psi_{\mathcal{S}}\right)\left(x^{\prime}\right)\right\}
$$

où $C_{\mathfrak{g}, \mathfrak{g}^{\prime}}=16$.

3.4.3. Le calcul de Chc pour $x^{\prime} \in \mathfrak{h}_{\mathcal{S}}^{\prime}$. - On suppose ici que $G^{\prime}=U(1,1)$, on peut identifier $\mathfrak{g}$ et $\mathfrak{g}^{\prime}$.

Proposition 3.4.16. - Pour $x^{\prime} \in \mathfrak{h}_{\mathcal{S}}^{\text {reg }}$, on a :

$$
\operatorname{Chc}(\psi)_{\mathcal{S}}\left(x^{\prime}\right)=\mathfrak{C}_{\mathfrak{g}, \mathfrak{g}^{\prime}, \mathfrak{h}_{\mathcal{S}}^{\prime}} \psi_{\mathcal{S}}\left(x^{\prime}\right) \text {. }
$$

Démonstration. - On a, avec les notations de la section 2.2.3, $V_{s}^{\prime}=V^{\prime}, V_{c}^{\prime}=$ $U=\{0\}$ et $\mathfrak{n}=\mathfrak{n}^{\prime}$. Grâce au théorème 2.2.7, on obtient pour $x^{\prime} \in \mathfrak{h}_{\mathcal{S}}^{\text {'reg }}$ :

$$
\operatorname{Chc}(\psi)\left(x^{\prime}\right)=\frac{\mathfrak{C}_{\mathfrak{g}, \mathfrak{g}^{\prime}, \mathfrak{h}_{\mathcal{S}}^{\prime}}}{\left|\operatorname{det}\left(\left.\operatorname{ad}\left(x^{\prime}\right)\right|_{\mathfrak{n}^{\prime}}\right)\right|} \int_{G L\left(X^{\prime}\right) / H_{s}^{\prime}} \psi_{\mathfrak{n}}^{K}\left(g x_{s}^{\prime} g^{-1}\right) d \dot{g},
$$

d'où

$$
\operatorname{Chc}(\psi)_{\mathcal{S}}\left(x^{\prime}\right)=\left|\pi_{\mathfrak{h}_{\mathcal{S}}^{\prime}}\left(x^{\prime}\right)\right| \operatorname{Chc}(\psi)\left(x^{\prime}\right)=\mathfrak{C}_{\mathfrak{g}, \mathfrak{g}^{\prime}, \mathfrak{h}_{\mathcal{S}}^{\prime}} \psi_{\mathcal{S}}\left(x^{\prime}\right) .
$$

On en déduit le résultat.

\subsection{Propriétés de Chc pour $G$ compact ou non}

On considère ici une des paires

$$
(U(2), U(2)),(U(2), U(1,1)),(U(1,1), U(2)) \text { ou }(U(1,1), U(1,1))
$$

que l'on note $\left(G, G^{\prime}\right)$. 
THÉORÈme 3.5.1. - Soit $\psi \in \mathcal{D}(\mathfrak{g})$, alors $\mathbf{C h c}(\psi)$ vérifie les propriétés suivantes :

(1) La fonction $\mathbf{C h c}(\psi)$ est une fonction lisse sur $\mathfrak{g}^{\text {reg }}$ et $G^{\prime}$-invariante.

(2) Pour toute partie compacte $\mathcal{K}$ de $\mathfrak{h}_{\varnothing}^{\prime}$ et tout $w \in \operatorname{Sym}\left(\mathfrak{h}_{\varnothing, \mathbb{C}}^{\prime}\right)$, on a :

$$
\sup _{x^{\prime} \in \mathcal{K} \cap \mathfrak{h}_{\varnothing}^{\prime r e g}}\left|\partial(w) \mathbf{C h c}(\psi)_{\varnothing}\left(x^{\prime}\right)\right|<\infty .
$$

(3) Si $G^{\prime}=U(2)$, la fonction $\mathbf{C h c}(\psi)_{\varnothing}$ se prolonge en une fonction lisse sur $\mathfrak{h}_{\varnothing}^{\prime}$.

(4) Si $G^{\prime}=U(1,1)$,

(a) Si $G=U(2)$, on a $\operatorname{Chc}(\psi)_{\mathcal{S}}=0$ et pour $w \in \operatorname{Sym}\left(\mathfrak{h}_{\varnothing, \mathbb{C}}^{\prime}\right)$, on a

$$
\left\langle\partial(w) \mathbf{C h c}(\psi)_{\varnothing}\right\rangle=0 .
$$

(b) Si $G=U(1,1)$, la fonction $\mathbf{C h c}(\psi)_{\mathcal{S}}$ se prolonge en une fonction lisse sur $\mathfrak{h}_{\mathcal{S}}^{\prime} \grave{a}$ support compact et pour $w \in \operatorname{Sym}\left(\mathfrak{h}_{\varnothing, \mathbb{C}}^{\prime}\right)$, on a

$$
\left\langle\partial(w) \mathbf{C h c}(\psi)_{\varnothing}\right\rangle(v)=\mathrm{i} \frac{(8 \pi)^{2}}{\mathfrak{C}_{\mathfrak{g}, \mathfrak{g}^{\prime}, \mathfrak{h}_{\mathcal{S}}^{\prime}}} \partial\left(c_{\mathcal{S}}(w)\right) \mathbf{C h c}(\psi)_{\mathcal{S}}(0, v) .
$$

Démonstration. - Montrons tout d'abord la première partie du point (1). Soient $\mathfrak{h}_{1}^{\prime}$, $\mathfrak{h}_{2}^{\prime}$ deux sous-algèbres de Cartan de $\mathfrak{g}^{\prime}$ conjuguées par $g \in G^{\prime}\left(\right.$ i.e. $\left.\mathfrak{h}_{1}^{\prime}=g \cdot \mathfrak{h}_{2}^{\prime}\right)$. En conservant les notations de la sous-section 2.1.1, on a $\mathfrak{a}_{1}^{\prime \prime}=g \cdot \mathfrak{a}_{2}^{\prime \prime}$ et $A_{1}^{\prime \prime \prime}=g \cdot A_{2}^{\prime \prime \prime}$; ainsi, on obtient avec les notations de la proposition 1.1.6 pour $\psi \in \mathcal{D}\left(\mathfrak{a}_{1}^{\prime \prime}\right)$ :

$$
\operatorname{Chc}(\psi)=\operatorname{Chc}\left(\psi^{g}\right)
$$

où $\psi^{g} \in \mathcal{D}\left(\mathfrak{a}_{2}^{\prime \prime}\right)$ est définie par $\psi^{g}(x)=\psi(g \cdot x)$. Pour $x^{\prime} \in \mathfrak{h}_{1}^{\text {reg }}$, l'opération de restriction de ces distributions respectivement à $x^{\prime}+\mathfrak{g}$ et $g \cdot x^{\prime}+\mathfrak{g}$ fournit l'égalité :

$$
\operatorname{Chc}(\psi)\left(x^{\prime}\right)=\mathbf{C h c}(\psi)\left(g \cdot x^{\prime}\right) \quad \forall \psi \in \mathcal{D}(\mathfrak{g})
$$

Si $G^{\prime}=U(2)$, on a $\mathfrak{g}^{\text {reg }}=G^{\prime} \cdot \mathfrak{h}_{\varnothing}^{\text {reg }}$ et d'après les propositions 3.2.1 et 3.4.15, la fonction $\mathbf{C h c}(\psi)_{\varnothing}$ est une fonction lisse ainsi $\mathbf{C h c}(\psi)$ est une fonction lisse sur $\mathfrak{g}^{\text {reg }}$. Si $G^{\prime}=U(1,1)$, on a $\mathfrak{g}^{\text {reg }}=G^{\prime} \cdot \mathfrak{h}_{\varnothing}^{\text {reg }} \amalg G^{\prime} \cdot \mathfrak{h}_{\mathcal{S}}^{\text {reg }}$ et d'après les propositions 3.2.3 et 3.4.15, les fonctions $\mathbf{C h c}(\psi)_{\varnothing}$ et $\mathbf{C h c}(\psi)_{\mathcal{S}}$ sont lisses. On en déduit le point (1). Le point (2) découle de la proposition $3.4 .15 \mathrm{si} G=U(1,1)$ et des propositions 3.2 .1 et $3.2 .3 \mathrm{si}$ $G=U(2)$. Supposons à présent que $G^{\prime}=U(2)$. D'après la proposition 3.2.1, on a le point (3) si $G=U(2)$. Si $G=U(1,1)$, d'après la proposition 3.4.15, comme $\varepsilon=-1$, le saut de la fonction $\partial(w) \mathbf{C h c}(\psi)_{\varnothing}$ est nul pour tout $w \in \operatorname{Sym}\left(\mathfrak{h}_{\varnothing, \mathbb{C}}\right)$ ainsi, $\operatorname{Chc}(\psi)_{\varnothing}$ se prolonge en une fonction lisse sur $\mathfrak{h}_{\varnothing}^{\prime}$. On en déduit le point (3). Supposons enfin $G^{\prime}=U(1,1)$, le première partie du point (4.a) découle de la proposition 3.2.3. D'après cette proposition, on sait aussi que $\mathbf{C h c}(\psi)_{\varnothing}$ se prolonge en une fonction lisse sur $\mathfrak{h}_{\varnothing}^{\prime}$. On en déduit l'égalité

$$
\left\langle\partial(w) \mathbf{C h c}(\psi)_{\varnothing}\right\rangle(v)=\mathrm{i} \partial\left(c_{\mathcal{S}}(w)\right) \mathbf{C h c}(\psi)_{\mathcal{S}}(0, v)=0 .
$$


Pour $G=U(1,1)$, la première partie du point (4.b) découle de la proposition 3.4.16.

Puis, pour $n, m \in \mathbb{N}$, on a

$$
\begin{aligned}
\left\langle\partial_{\alpha}^{n} \partial_{\beta}^{m}\right\rangle \mathbf{C h c}(\psi)_{\varnothing} & =\left\langle\partial_{\alpha}^{n} \mathbf{C h c}\left(\partial_{z}^{m} \psi\right)_{\varnothing}\right\rangle(v) \\
& =\mathrm{i} \frac{(8 \pi)^{2}}{\mathfrak{C}_{\mathfrak{g}, \mathfrak{g}^{\prime}, \mathfrak{h}_{\mathcal{S}}^{\prime}}} \partial_{u}^{n} \mathbf{C h c}\left(\partial_{z}^{m} \psi\right)_{\mathcal{S}}(0, v) \\
& =\mathrm{i} \frac{(8 \pi)^{2}}{\mathfrak{C}_{\mathfrak{g}, \mathfrak{g}^{\prime}, \mathfrak{h}_{\mathcal{S}}^{\prime}}} \partial_{u}^{n} \partial_{v}^{m} \mathbf{C h c}(\psi)_{\mathcal{S}}(0, v) .
\end{aligned}
$$

On en déduit le résultat.

Corollaire 3.5.2. - On suppose que $\left(G, G^{\prime}\right)=(U(1,1), U(1,1))$. On a

$$
\partial(w) \mathbf{C h c}(\psi)_{\varnothing}(\beta)=\mathrm{i} \partial\left(c_{\mathcal{S}}(w)\right) \mathbf{C h c}(\psi)_{\mathcal{S}}(0, \beta)
$$

si et seulement si

$$
\mathfrak{C}_{\mathfrak{g}, \mathfrak{g}^{\prime}, \mathfrak{h}_{\mathcal{S}}^{\prime}}=(8 \pi)^{2}
$$





\section{CHAPITRE 4}

\section{PROPRIÉTÉS DES INTÉGRALES INVARIANTES}

Dans ce chapitre, après avoir étendu les notations sur les sous-algèbres de Cartan, on rappelle la définition et les propriétés de l'intégrale invariante de Harish-Chandra, sous-section 4.1.2. On montre ensuite que celle-ci se décompose en une somme finie de fonctions qui ne possèdent des singularités que sur certains hyperplans, Théorème 4.3.11, puis on montre que ces fonctions peuvent être approchées par des produits d'intégrales invariantes sur des sous-algèbres réductives de rang inférieur à 2 , Théorème 4.4.4. Dans la section 4.2, on obtient une nouvelle formulation de Chc pour une sous-algèbre de Cartan compacte. Ce chapitre est indépendant des précédents.

Dans ce chapitre, on note $I=\{1, \ldots, \mathrm{p}\}$ et $J=\{\mathrm{p}+1, \ldots, \mathrm{n}\}$.

\subsection{L'intégrale invariante de Harish-Chandra}

Dans une première sous-section, on construit une famille de sous-algèbres de Cartan $\left(\mathfrak{h}_{M}\right)_{M \in \Omega}$ où $\Omega$ s'identifie avec l'ensemble des systèmes de racines admissibles de $\Phi^{\text {nc }}\left(\mathfrak{h}_{\varnothing}\right)$ puis, on détermine le groupe de Weyl associé à chacune de ces sous-algèbres de Cartan. Dans la deuxième sous-section, on définie l'intégrale invariante de HarishChandra sur $\mathfrak{h}_{M}^{\text {reg }}$ pour $M \in \Omega$ et on rappelle les propriétés de ces fonctions. Dans la dernière sous-section, on obtient une reformulation du corollaire 2.2.5.

\subsubsection{Propriétés des sous-algèbres de Cartan}

Notations et définitions. - On désigne par $\Omega$ l'ensemble des injections $L$ d'une partie de $I$ dans $J$. On note pour $L \in \Omega, \mathrm{A}_{L}$ le domaine de définition de $L$. Deux éléments $L$ et $M$ de $\Omega$ seront dits disjoints si $\mathrm{A}_{L} \cap \mathrm{A}_{M}=\varnothing$ et $\operatorname{im}(L) \cap \operatorname{im}(M)=\varnothing$. On notera alors $L+M \in \Omega$ le prolongement de $L$ par $M$; cette opération est associative et commutative. Pour un couple $(i, j) \in I \times J$, on note $[i, j]$ l'élément de $\Omega$ qui vérifie $\mathrm{A}_{[i, j]}=\{i\}$ et $[i, j](i)=j$.

À chaque élément $L$ de $\Omega$, on associe le système de racines imaginaires non compactes $\mathcal{S}_{L}=\left\{e_{j}-e_{L(j)} \mid j \in \mathrm{A}_{L}\right\} \subset \Phi^{\mathrm{nc}}\left(\mathfrak{h}_{\varnothing}\right)$, ainsi une partie $\mathcal{S}$ de $\Phi^{\mathrm{nc}}\left(\mathfrak{h}_{\varnothing}\right)$ est 
fortement orthogonale si et seulement si elle est associée à un élément $L \in \Omega$. On note $I_{L}=I \backslash \mathrm{A}_{L}$ et $J_{L}=J \backslash \operatorname{im}(L)$. Pour $(a, b) \in I \times J$, on pose

$$
P_{a, b}=E_{a, b}+E_{b, a} \text { et } A_{a, b}=\mathrm{i}\left(E_{a, a}+E_{b, b}\right) .
$$

On associe à $L$ l'élément $\mathfrak{h}_{L}$ de $\operatorname{Car}(\mathfrak{g})$ défini ainsi

$$
\mathfrak{h}_{L}=\underset{j \in \mathrm{A}_{L}}{\oplus} \mathbb{R} P_{j, L(j)} \underset{j \in \mathrm{A}_{L}}{\oplus} \mathbb{R} A_{j, L(j)} \underset{j \in I_{L} \cup J_{L}}{\oplus} \mathrm{iR} E_{j, j}
$$

On considère sur la sous-algèbre de Cartan $\mathfrak{h}_{L}$ le système de coordonnées $\left(u_{j}\right)_{j \in \mathrm{A}_{L}}$, $\left(v_{j}\right)_{j \in \mathrm{A}_{L}}$ et $\left(x_{j}\right)_{j \in I_{L} \cup J_{L}}$. Pour $(a, b) \in I \times J$, on définit la transformée de Cayley $c_{a, b}$ de $\mathbb{C} E_{a, a} \oplus \mathbb{C} E_{b, b}$ dans $\mathbb{C} P_{a, b} \oplus \mathbb{C} A_{a, b}$ dont la matrice dans les bases $\left(\mathrm{i} E_{a, a}, \mathrm{i} E_{b, b}\right)$ et $\left(P_{a, b}, A_{a, b}\right)$ est

$$
\frac{1}{2}\left(\begin{array}{cc}
\mathrm{i} & -\mathrm{i} \\
1 & 1
\end{array}\right)
$$

Si $[i, j]$ et $L$ sont disjoints, l'application $c_{i, j}$ s'étend de $\mathfrak{h}_{L, \mathbb{C}}$ dans $\mathfrak{h}_{L+[i, j], \mathbb{C}}$ en posant $c_{i, j}\left(E_{p, p}\right)=E_{p, p}$ pour $p \in\{1, \ldots, \mathrm{n}\} \backslash\{i, j\}$. Soit $[a, b]$ disjoint à la fois avec $[i, j]$ et $L$; les éléments $L+[a, b]$ et $(L+[a, b])+[i, j]=(L+[i, j])+[a, b]$ sont définis et on a le diagramme commutatif suivant :

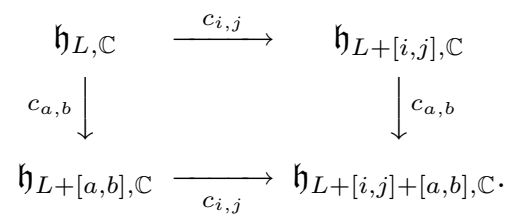

Ce diagramme permet de définir $\operatorname{sur} \mathfrak{h}_{L, \mathbb{C}}$ pour un élément $M \in \Omega$ disjoint de $L$ l'application

$$
c_{M}^{L}=\prod_{j \in A_{M}} c_{j, M(j)} .
$$

Si $L^{\prime} \in \Omega$ est tel que $M$ et $L^{\prime}$ soient disjoints alors pour $x \in \mathfrak{h}_{L, \mathbb{C}} \cap \mathfrak{h}_{L^{\prime}, \mathbb{C}}$, on a $c_{M}^{L}(x)=c_{M}^{L^{\prime}}(x)$; ce qui permet de poser $c_{M}(x)=c_{M}^{L}(x)$. On considère sur $\Omega$ l'action naturelle du groupe $\mathfrak{S}(I) \times \mathfrak{S}(J)$.

Proposition 4.1.1. - On a les propriétés suivantes :

(1) Pour $L, M \in \Omega$ disjoints, on a $c_{L}: \mathfrak{h}_{M, \mathbb{C}} \stackrel{\sim}{\rightarrow} \mathfrak{h}_{L+M, \mathbb{C}}$.

(2) L'ensemble quotient $\Omega /(\mathfrak{S}(I) \times \mathfrak{S}(J))$ est isomorphe avec l'ensemble des classes de conjugaison de sous-algèbres de Cartan de $\mathfrak{g}$ par l'application

$$
L \longmapsto \mathfrak{h}_{L}
$$

Démonstration. — Le point (1) découle des propriétés énoncées ci-dessus. Pour le point (2), on peut remarquer que deux éléments $L$ et $M$ de $\Omega$ sont conjugués par $\mathfrak{S}(I) \times \mathfrak{S}(J)$ si seulement si $\operatorname{Card}\left(\mathrm{A}_{L}\right)=\operatorname{Card}\left(\mathrm{A}_{M}\right)$ ce qui équivaut à dire que $\mathfrak{h}_{L}$ et $\mathfrak{h}_{M}$ ont des parties déployées de même dimension donc sont conjuguées d'après $[\mathbf{8}$, p. 400]. 
Remarque. - On a en particulier $c_{L}\left(\mathfrak{h}_{\varnothing, \mathbb{C}}\right) \cap \mathfrak{g}=\mathfrak{h}_{L}$ pour $L \in \Omega$.

On note $H_{N}$ le sous-groupe de Cartan de $G$ d'algèbre de Lie $\mathfrak{h}_{N}$ pour $N \in \Omega$. L'application

$$
\begin{aligned}
\theta: \mathfrak{g} & \longrightarrow \mathfrak{g} \\
x & \longmapsto-{ }^{t} \bar{x}
\end{aligned}
$$

définie une involution de Cartan de $\mathfrak{g}$. Les sous-algèbres de Cartan $\mathfrak{h}_{N}$ sont $\theta$-stables. On pose

$$
\mathfrak{t}_{N}=\left\{x \in \mathfrak{h}_{N} \mid \theta(x)=x\right\} \text { et } \mathfrak{a}_{N}=\left\{x \in \mathfrak{h}_{N} \mid \theta(x)=-x\right\} .
$$

Propriétés du groupe de Weyl de $H_{N}$. — Dans ce paragraphe, on utilise les notations introduites dans [7]. On désigne par $\mathcal{S}$ le système de racines fortement orthogonales associé à $M \in \Omega$. On considère les sous-groupes suivants du groupe de Weyl de $H_{M}$, $W\left(H_{M}\right)$ (cf. [7, (2.35) et (2.25)] pour plus de détails).

$$
\begin{aligned}
W\left(H_{M}\right)_{-} & =\left\langle s_{\alpha}\left|\alpha \in \Phi\left(\mathfrak{h}_{M}\right), \alpha\right|_{\mathfrak{t}_{M}}=0\right\rangle \\
U\left(H_{M}\right) & =\left\{c_{M} \circ w \circ c_{M}^{-1} \mid w \in W\left(H_{\varnothing}\right), w \mathcal{S} \subset \mathcal{S} \cup-\mathcal{S}\right\}
\end{aligned}
$$

où $s_{\alpha}$ désigne la réflexion associée à $\alpha$. On considère pour $\sigma \in \mathfrak{S}(I \cup J)$ l'élément $w_{\sigma}$ de $W\left(\mathfrak{h}_{\varnothing, \mathbb{C}}\right)$ (le groupe de Weyl de $\mathfrak{h}_{\varnothing, \mathbb{C}}$ en tant que sous-algèbre de Cartan de $\mathfrak{g}_{\mathbb{C}}$ ) qui vérifie $\left(w_{\sigma}(x)\right)_{i}=x_{\sigma^{-1}(i)}$ pour $x \in \mathfrak{h}_{\varnothing, \mathbb{C}}$ et $i \in\{1, \ldots, \mathrm{n}\}$. On sait que l'application

$$
\begin{aligned}
\zeta: \mathfrak{S}(I \cup J) & \longrightarrow W\left(\mathfrak{h}_{\varnothing, \mathbb{C}}\right) \\
\sigma & \longmapsto w_{\sigma}
\end{aligned}
$$

est un isomorphisme de groupes dont la restriction à $\mathfrak{S}(I) \times \mathfrak{S}(J)$ induit un isomorphisme de groupes avec $W\left(H_{\varnothing}\right)$. On note aussi $W\left(\mathfrak{h}_{M, \mathbb{C}}\right)$ le groupe de Weyl de $\mathfrak{h}_{M, \mathbb{C}}$ en tant que sous-algèbre de Cartan de $\mathfrak{g}_{\mathbb{C}}$ et on considère le morphisme de groupes $\zeta^{\prime}: \mathfrak{S}(I \cup J) \longrightarrow W\left(\mathfrak{h}_{M, \mathbb{C}}\right)$ défini par

$$
\zeta^{\prime}(\sigma)=c_{M} \circ \zeta(\sigma) \circ c_{M}^{-1} .
$$

Proposition 4.1.2 ([7, Proposition 2.36]). — L'application

$$
\begin{aligned}
W\left(H_{M}\right)_{-} \times U\left(H_{M}\right) & \longrightarrow W\left(H_{M}\right) \\
\left(w_{1}, w_{2}\right) & \longmapsto w_{1} w_{2}
\end{aligned}
$$

est surjective. On a le lemme suivant :

LEMME 4.1.3. - Le groupe $U\left(H_{M}\right)$ s'identifie par $\zeta^{\prime} \grave{a}$

$$
\left\{\sigma \in \mathfrak{S}(I) \times \mathfrak{S}(J) \mid \sigma\left(\mathrm{A}_{M}\right)=\mathrm{A}_{M} \text { et } M \circ \sigma(i)=\sigma \circ M(i) \forall i \in \mathrm{A}_{M}\right\} .
$$

Démonstration. - Pour un élément $w \in W\left(H_{\varnothing}\right)$, on a l'équivalence suivante

(1) $c_{M} \circ w \circ c_{M}^{-1} \in U\left(H_{M}\right)$

(2) $w$ permute les hyperplans $\left\{x_{i}=x_{M(i)}\right\}$ avec $i \in \mathrm{A}_{M}$.

On en déduit alors le résultat. 
LEMME 4.1.4. — Le groupe $W\left(H_{M}\right)_{-}$s'identifie par $\zeta^{\prime} \grave{a}$

$$
\left\langle(i, M(i)) \mid i \in \mathrm{A}_{M}\right\rangle \subset \mathfrak{S}(I \cup J) .
$$

Démonstration. - Les racines $\alpha \in \Phi\left(\mathfrak{h}_{M}\right)$ telle que $\left.\alpha\right|_{\mathfrak{t}_{M}}=0$ sont les racines de la forme $c_{M} \circ \beta \circ c_{M}^{-1}$ avec $\beta \in \mathcal{S}$ ainsi $W\left(H_{M}\right)$ s'identifie par $\zeta$ à $\left\langle(i, M(i)) \mid i \in \mathrm{A}_{M}\right\rangle$.

Proposition 4.1.5. - On a

$$
\begin{gathered}
\operatorname{Card}\left\{W\left(H_{M}\right)\right\}=(\mathrm{p}-m) !(\mathbf{q}-m) ! m ! 2^{m} \\
\operatorname{Card}\left\{\left.w\right|_{\mathfrak{a}_{M}} \mid w \in W\left(H_{M}\right)\right\}=m ! 2^{m}
\end{gathered}
$$

où $m=\operatorname{Card}\left(\mathrm{A}_{M}\right)$.

Démonstration. — D'après les lemmes 4.1 .3 et 4.1 .4 , on a

$$
U\left(H_{M}\right) \cap W\left(H_{M}\right)_{-}=\{i d\}
$$

ainsi en utilisant la proposition 4.1.2, on obtient

$$
\operatorname{Card}\left\{W\left(H_{M}\right)\right\}=(\mathrm{p}-m) !(\mathrm{q}-m) ! m ! 2^{m} .
$$

D'après le lemme 4.1.3, $U\left(H_{M}\right)$ s'identifie à

$$
\mathfrak{S}\left(I_{M}\right) \times \mathfrak{S}\left(J_{M}\right) \times \mathfrak{S}\left(\mathrm{A}_{M}\right) .
$$

On observe que pour $\sigma \in \mathfrak{S}\left(I_{M}\right) \times \mathfrak{S}\left(J_{M}\right),\left.w_{\sigma}\right|_{\mathfrak{a}_{M}}=i d$. Puis, pour $\sigma \in \mathfrak{S}\left(\mathrm{A}_{M}\right)$ et $u=\left(u_{i}\right)_{i \in \mathrm{A}_{M}} \in \mathfrak{a}_{M}$, on a pour $w_{\sigma} \in U\left(H_{M}\right)$

$$
\left(w_{\sigma} \circ c_{M}^{-1}(u)\right)_{i}= \begin{cases}\left(c_{M}^{-1}(u)\right)_{i}=0 & \text { si } i \in I_{M} \cup J_{M} \\ \left(c_{M}^{-1}(u)\right)_{\sigma^{-1}(i)}=-\mathrm{i} u_{\sigma^{-1}(i)}=c_{M}^{-1}(\sigma \cdot u)_{i} & \text { si } i \in \mathrm{A}_{M} \\ \left(c_{M}^{-1}(u)\right)_{M \circ \sigma^{-1} \circ M^{-1}(i)}=c_{M}^{-1}(\sigma \cdot u)_{i} & \text { si } i \in \operatorname{im}(M)\end{cases}
$$

où $(\sigma \cdot u)_{i}=u_{\sigma^{-1}(i)}$ pour $i \in \mathrm{A}_{M}$. Ainsi, on en déduit que

$$
\begin{aligned}
\mathfrak{S}\left(\mathbf{A}_{M}\right) & \longrightarrow\left\{\left.w\right|_{\mathfrak{a}_{M}} \mid w \in W\left(H_{M}\right)\right\} \\
\sigma & \left.\longmapsto w_{\sigma}\right|_{\mathfrak{a}_{M}}
\end{aligned}
$$

est injectif. Comme

$$
\left\{\left.w_{\sigma}\right|_{\mathfrak{a}_{M}} \mid \sigma \in \mathfrak{S}\left(\mathrm{A}_{M}\right)\right\} \cap\left\{\left.w\right|_{\mathfrak{a}_{M}} \mid w \in W\left(H_{M}\right)_{-}\right\}=\varnothing,
$$

on obtient

$$
\operatorname{Card}\left\{\left.w\right|_{\mathfrak{a}_{M}} \mid w \in W\left(H_{M}\right)\right\}=\operatorname{Card} \mathfrak{S}\left(\mathbf{A}_{M}\right) \operatorname{Card} W\left(H_{M}\right)_{-}=m ! 2^{m} .
$$




\subsubsection{Définition et propriétés de l'intégrale invariante de Harish-Chandra}

DÉfinition 4.1.6. - Soit $\phi \in \mathcal{D}(\mathfrak{g}), M \in \Omega$ et $\mathcal{S}$ le système de racines fortement orthogonales associé à $M$. On considère la fonction $\phi_{M}$ définie sur $\mathfrak{h}_{M}^{\text {reg }}$ par

$$
\phi_{M}(x)=\overline{\pi_{\varnothing}}\left(c_{M}^{-1}(x)\right) \mathbf{e}_{M}(x) \int_{G / H_{M}} \phi(g \cdot x) d \dot{g}
$$

où $\mathbf{e}_{M}(x)=\operatorname{sign}\left(\prod_{\alpha \in \mathcal{S}} \bar{\alpha}\left(c_{M}^{-1}(x)\right)\right)$.

On appelle cette fonction l'intégrale invariante de Harish-Chandra. Il existe un morphisme de groupes

$$
\varepsilon_{M}: W\left(H_{M}\right) \longrightarrow\{ \pm 1\}
$$

tel que

$$
\overline{\pi_{\varnothing}}\left(c_{M}^{-1}(\sigma \cdot x)\right) \mathbf{e}_{M}(\sigma \cdot x)=\varepsilon_{M}(\sigma) \overline{\pi_{\varnothing}}\left(c_{M}^{-1}(x)\right) \mathbf{e}_{M}(x)
$$

pour $\sigma \in W\left(H_{M}\right)$. Pour $M$ et $[a, b] \in \Omega$ disjoints, on remarque que l'on a l'égalité entre hyperplans

$$
\mathfrak{h}_{M} \cap\left\{x_{a}=x_{b}\right\}=\mathfrak{h}_{M+[a, b]} \cap\left\{u_{a}=0\right\} ;
$$

on considère l'ouvert $\mathfrak{h}_{M}^{(a, b)}$ de cet hyperplan défini ainsi

$$
\left\{x \in \mathfrak{h}_{M} \cap\left\{x_{a}=x_{b}\right\} \mid x_{i} \neq x_{j} \forall(i, j) \in I_{M} \times J_{M} \backslash\{(a, b)\}\right\} .
$$

Le théorème suivant énonce les propriétés de l'intégrale invariante de Harish-Chandra qui seront utilisées dans la suite.

ThÉORÈme 4.1.7. - Pour $\phi \in \mathcal{D}(\mathfrak{g})$, on a les propriétés suivantes :

(1) Pour $M \in \Omega$, la fonction $\phi_{M}$ est lisse et à support borné.

(2) Pour $M \in \Omega, w \in \operatorname{Sym}\left(\mathfrak{h}_{M, \mathbb{C}}\right)$ et $\mathcal{K}$ une partie compacte de $\mathfrak{h}_{M}$, on a

$$
\sup _{x \in \mathfrak{h}_{M}^{\text {reg }} \cap \mathcal{K}}\left|\partial(w) \phi_{M}(x)\right|<\infty .
$$

(3) Pour $M \in \Omega$ et $w \in S y m\left(\mathfrak{h}_{M, \mathbb{C}}\right), \partial(w) \phi_{M}$ se prolonge par continuité sur $\mathfrak{h}_{M}^{\text {nc }}$.

(4) Pour $M,[a, b] \in \Omega$ disjoints et $w \in \operatorname{Sym}\left(\mathfrak{h}_{M, \mathbb{C}}\right)$, on $a$

$$
\left\langle\partial(w) \phi_{M}\right\rangle_{a, b}(x)=\mathrm{i} \partial\left(c_{a, b}(w)\right) \phi_{M+[a, b]}(x)
$$

pour $x \in \mathfrak{h}_{M}^{(a, b)}$.

(5) Pour $\sigma \in W\left(H_{M}\right)$ et $x \in \mathfrak{h}_{M}^{\mathrm{reg}}$, on $a$

$$
\psi_{M}(\sigma \cdot x)=\varepsilon_{M}(\sigma) \psi_{M}(x) .
$$




\subsection{Propriétés générales de Chc}

Les notations introduites au début de cette partie permettent de nouvelles formulations du corollaire 2.2.5. Le principal résultat de cette section est la proposition 4.2.2 qui sera utilisée dans la partie suivante.

On rappelle que $\mathrm{n}$ et $\mathrm{n}^{\prime}$ désignent deux entiers tels que $\mathrm{n}^{\prime} \leqslant \mathrm{n}$. On note $\mathcal{I}$ l'ensemble des injections de $\left\{1, \ldots, n^{\prime}\right\}$ dans $\{1, \ldots, n\}$. On généralise les notations utilisées dans la sous-section 2.2 .2 ; pour $M \in \Omega, L \in \mathcal{I}, \psi \in \mathcal{D}(\mathfrak{g})$ et $x^{\prime} \in \mathfrak{h}_{M}^{\text {/reg }}$ on pose

$$
\nu_{L, x^{\prime}, M}^{t}(\psi)(x)=P_{L, p}\left(c_{M}^{-1}(x)\right) \operatorname{chc}\left(c_{M}^{-1}(x)+\mathrm{i} t y_{M}^{L}-x^{\prime}\right) \psi_{M}(x) \mu_{M}
$$

où les définitions de chc et $P_{L}$ sont inchangées et $y_{M}^{L} \in \mathfrak{h}_{\varnothing}$ vérifie

$$
y_{M k}^{L}= \begin{cases}\operatorname{sign}\left(V_{k}\right) \operatorname{sign}\left(V_{i}^{\prime}\right) & \text { si } L(i)=k \text { et } k \in I_{M} \cup J_{M}, \\ 0 & \text { sinon. }\end{cases}
$$

On pose aussi

$$
\mathfrak{h}_{M}^{+}=\mathfrak{t}_{M}+\mathfrak{a}_{M}^{+}
$$

où $\mathfrak{a}_{M}^{+}$représente une chambre de Weyl de $\mathfrak{a}_{M}$ telle $\mathbf{e}_{M}$ soit positive sur celle-ci. Le corollaire 2.2.5 devient alors avec ces notations

$$
M_{p, \mathfrak{h}_{\varnothing}^{\prime}}\left(x^{\prime}\right) \overline{\pi_{\mathfrak{h}_{\varnothing}^{\prime}}}\left(x^{\prime}\right) \mathbf{C h c}(\psi)\left(x^{\prime}\right)=C_{\mathfrak{g}, \mathfrak{g}^{\prime}} \sum n_{M} \lim _{t \rightarrow 0, t>0} \int_{\mathfrak{h}_{M}^{+}} \nu_{L, x^{\prime}, M}^{t}(\psi)
$$

où la somme se fait sur un système de représentants de sous-algèbres de Cartan de $\mathfrak{g}$ et sur l'ensemble des injections $L \in \mathcal{I}$. L'entier $n_{M}$ est égal à

$$
\frac{\operatorname{Card}\left\{\left.w\right|_{\mathfrak{a}_{M}} \mid w \in W\left(H_{M}\right)\right\}}{\operatorname{Card}\left(W\left(H_{M}\right)\right)}
$$

et $p \in\left\{0, \ldots, \mathrm{n}-\mathrm{n}^{\prime}\right\}$.

On obtient la nouvelle expression suivante.

Lemme 4.2.1. - Pour $x^{\prime} \in \mathfrak{h}_{\varnothing}^{\text {reg }}$, on $a:$

$$
M_{p, \mathfrak{h}_{\varnothing}^{\prime}}\left(x^{\prime}\right) \overline{\pi_{\mathfrak{h}_{\varnothing}^{\prime}}}\left(x^{\prime}\right) \mathbf{C h c}(\psi)\left(x^{\prime}\right)=\frac{C_{\mathfrak{g}, \mathfrak{g}^{\prime}}}{\mathrm{p} ! \mathfrak{q} !} \sum_{(M, L) \in \Omega \times \mathcal{I}} m ! \lim _{t \rightarrow 0, t>0} \int_{\mathfrak{h}_{M}^{+}} \nu_{L, x^{\prime}, M}^{t} .
$$

où l'entier $m$ est égal à $\operatorname{Card}\left(\mathrm{A}_{M}\right)$.

Démonstration. - En posant $m=\operatorname{Card}\left(\mathrm{A}_{M}\right)$, on a les égalités suivantes d'après la proposition 4.1 .5

$$
\operatorname{Card}\left(W\left(H_{M}\right)\right)=(\mathrm{p}-m) !(\mathbf{q}-m) ! 2^{m} m !
$$

ainsi

$$
\begin{gathered}
n_{M}=\frac{1}{(\mathbf{p}-m) !(\mathbf{q}-m) !} \\
\operatorname{Card}\left\{\left.w\right|_{\mathfrak{a}_{M}} \mid w \in W\left(H_{M}\right)\right\}=m ! 2^{m} .
\end{gathered}
$$


Le centralisateur de $M \in \Omega$ sous l'action du groupe $\mathfrak{S}(I) \times \mathfrak{S}(J)\left(=W\left(H_{\varnothing}\right)\right)$ s'identifie au groupe $\mathfrak{S}\left(\mathrm{A}_{M}\right) \times \mathfrak{S}\left(J_{M}\right) \times \mathfrak{S}\left(I_{M}\right)$ ainsi, le cardinal de l'orbite de $M$ sous l'action de $\mathfrak{S}(I) \times \mathfrak{S}(J)$ est égal à

$$
\operatorname{Card}\{(\mathfrak{S}(I) \times \mathfrak{S}(J)) \cdot M\}=\frac{\mathrm{p} ! \mathrm{q} !}{m !(\mathrm{p}-m) !(\mathrm{q}-m) !},
$$

d'où

$$
\frac{n_{M}}{\operatorname{Card}\{(\mathfrak{S}(I) \times \mathfrak{S}(J)) \cdot M\}}=\frac{m !}{\mathrm{p} ! \mathrm{q} !} .
$$

On en déduit le résultat.

On pose :

$$
\check{\mathfrak{h}}_{M}=\left\{x \in \mathfrak{h}_{M} \mid \bar{\alpha}\left(c_{M}^{-1}(x)\right)>0 \forall \alpha \in \mathcal{S}\right\} .
$$

On obtient alors la nouvelle expression suivante :

Proposition 4.2.2. - Pour $x^{\prime} \in \mathfrak{h}_{\varnothing}^{\text {reg }}$, on $a:$

$$
M_{p, \mathfrak{h}_{\varnothing}^{\prime}}\left(x^{\prime}\right) \overline{\pi_{\mathfrak{h}_{\varnothing}^{\prime}}}\left(x^{\prime}\right) \mathbf{C h c}(\psi)\left(x^{\prime}\right)=\frac{C_{\mathfrak{g}, \mathfrak{g}^{\prime}}}{\mathrm{p} ! \mathfrak{q} !} \sum_{(M, L) \in \Omega \times \mathcal{I}} \lim _{t \rightarrow 0, t>0} \int_{\check{\mathfrak{h}}_{M}} \nu_{L, x^{\prime}, M}^{t}(\psi) .
$$

Démonstration. - On pose $m=\operatorname{Card}\left(\mathrm{A}_{M}\right)$. Les chambres de Weyl de la partie déployée $\mathfrak{a}_{M}$ de $\mathfrak{h}_{M}$ sont en bijections avec $\{ \pm 1\}^{m} \times \mathfrak{S}(\{1, \ldots, m\})$ par l'application suivante

$$
\begin{aligned}
\left(\left(\varepsilon_{i}\right)_{1 \leqslant i \leqslant m}, \sigma\right) \in\{ \pm 1\}^{m} \times \mathfrak{S} & (\{1, \ldots, m\}) \\
& \longrightarrow\left\{x \in \mathfrak{a}_{M} \mid 0<\varepsilon_{1} u_{\sigma(1)}<\cdots<\varepsilon_{m} u_{\sigma(m)}\right\} \subset \mathfrak{a}_{M}
\end{aligned}
$$

Les chambres de Weyl de $\mathfrak{a}_{M}$ contenues dans $\check{\mathfrak{h}}_{M}$ correspondent par l'application cidessus aux couples $\left((-1)_{1 \leqslant i \leqslant m}, \sigma\right)$ avec $\sigma \in \mathfrak{S}(\{1, \ldots, m\})$. On en déduit le résultat.

\subsection{Décomposition des intégrales invariantes}

L'objet de cette section est de montrer le théorème 4.3.11 et la proposition 4.3.14. Le premier de ces résultats montre que les intégrales invariantes de Harish-Chandra peuvent se décomposer en somme finie de fonctions qui ne possèdent des sauts que sur certains hyperplans. Puis la proposition 4.3.14 montre que cette décomposition est continue.

Dans cette sous-section $L$ désigne un élément de $\Omega, I_{L}=I \backslash \mathrm{A}_{L}$ et $J_{L}=J \backslash \operatorname{im}(L)$.

DÉfinition 4.3.1. - Pour $d \in \mathbb{N}$, on note $\mathcal{E}\left(\mathfrak{h}_{L}, d\right)$ le sous-espace de dimension finie de $\operatorname{Sym}\left(\mathfrak{h}_{L, \mathbb{C}}\right)$ constitué des éléments $w$ tels que

$$
\operatorname{deg}(w) \leqslant d
$$


Si $\Gamma$ est une partie de $I_{L} \times J_{L}$, on pose

$$
\mathfrak{h}_{L}^{\Gamma}=\left\{x \in \mathfrak{h}_{L} \mid x_{i} \neq x_{j} \forall(i, j) \in \Gamma\right\} .
$$

Une fonction $\phi$ définie sur $\mathfrak{h}_{L}^{\Gamma}$ est dite de classe $C^{d}$ si elle possède une dérivée $\partial(w) \phi$ continue $\operatorname{sur} \mathfrak{h}_{L}^{\Gamma}$ pour tout $w \in \mathcal{E}\left(\mathfrak{h}_{L}, d\right)$. Pour $L \in \Omega$, on pose

$$
\Gamma_{L}=\left\{(i, L(i)) \mid i \in \mathrm{A}_{L}\right\} .
$$

On considère la racine positive $\alpha=\left(e_{i}-e_{j}\right) \circ c_{L}^{-1},(i<j)$ de $\mathfrak{h}_{L}$. La nature réelle, imaginaire compacte, imaginaire non compacte ou complexe de $\alpha$ se détermine ainsi

(1) La racine $\alpha$ est réelle si et seulement si $(i, j) \in \Gamma_{L}$.

(2) La racine $\alpha$ est imaginaire compacte si et seulement si $(i, j) \in\left(I_{L} \times I_{L}\right) \cup$ $\left(J_{L} \times J_{L}\right)$.

(3) La racine $\alpha$ est imaginaire non compacte si et seulement si $(i, j) \in I_{L} \times J_{L}$.

(4) La racine $\alpha$ est complexe sinon.

REMARQUE. - On a en particulier $\mathfrak{h}_{L}^{I_{L} \times J_{L}}=\mathfrak{h}_{L}^{\text {nc }}$.

Pour $(a, b) \in I_{L} \times J_{L}$, on note $s_{a, b}$ la réflexion de $\mathfrak{h}_{M}$ par rapport à l'hyperplan $\left\{\alpha_{a, b}=0\right\}$ telle que

$$
s_{a, b}\left(\mathrm{i} E_{a, a}-\mathrm{i} E_{b, b}\right)=\mathrm{i} E_{b, b}-\mathrm{i} E_{a, a} .
$$

Cette réflexion induit un automorphisme de $\mathcal{E}\left(\mathfrak{h}_{L}, d\right)$ que l'on note aussi $s_{a, b}$. Pour $\Gamma \subset I_{L} \times J_{L}$, on note $\mathfrak{F}_{\Gamma}$ l'ensemble des applications de $\Gamma$ dans $\{ \pm 1\}$ et on considère pour $\varepsilon \in \mathfrak{F}_{\Gamma}$, le cône fermé de $\mathfrak{h}_{L}$

$$
C_{\varepsilon}^{L}=\left\{x \in \mathfrak{h}_{L} \mid \varepsilon(i, j)\left(x_{i}-x_{j}\right) \geqslant 0 \forall(i, j) \in \Gamma\right\} .
$$

Pour une partie (convexe) $A$ d'un $\mathbb{R}$-espace vectoriel de dimension finie, on note Int (A) l'intérieur de $A$ en tant que partie $\operatorname{de} \operatorname{Vect}(A)$ (le sous-espace vectoriel engendré par $A$ ), on a alors

$$
\mathfrak{h}_{L}^{\Gamma}=\coprod_{\varepsilon \in \mathfrak{F}_{\Gamma}} \operatorname{Int}\left(\mathrm{C}_{\varepsilon}^{\mathrm{L}}\right)
$$

DÉfinition 4.3.2. - Pour $\Gamma \subset I_{L} \times J_{L}$, on note $\mathcal{W}_{c}\left(\mathfrak{h}_{L}, d, \Gamma\right)$ le sous-espace de $\mathcal{H}\left(\mathfrak{h}_{L}^{\Gamma}, d\right)$ des fonctions $\theta$ telles que

(1) Pour $w \in \mathcal{E}\left(\mathfrak{h}_{L}, d\right)$ et $(i, j) \in \Gamma$ tel que $s_{i, j}(w)=-w$, la fonction $\partial(w) \theta$ se prolonge en une fonction continue sur $\mathfrak{h}_{L}^{\Gamma \backslash\{(i, j)\}}$.

(2) Pour $i \in \mathrm{A}_{L}, \theta$ est une fonction paire en $u_{i}{ }^{(1)}$.

Pour $\varepsilon \in \mathfrak{F}_{\Gamma}$, on note le prolongement par continuité de $\partial(w) \theta$ à $C_{\varepsilon}^{L}$ par $\left.\partial(w) \theta\right|_{C_{\varepsilon}^{L}}$. Pour $(i, j) \in I_{L} \times J_{L}$, on remarque que l'on a l'égalité suivante

$$
\mathfrak{h}_{L+[i, j]} \cap\left\{u_{i}=0\right\}=\mathfrak{h}_{L} \cap\left\{x_{i}=x_{j}\right\}=\mathfrak{h}_{L} \cap \mathfrak{h}_{L+[i, j]}
$$

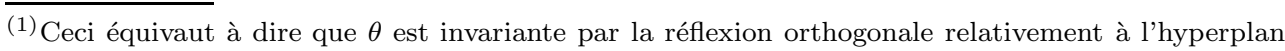
$\left\{u_{i}=0\right\}$.
} 
Pour $\varepsilon \in \mathfrak{F}_{\Gamma}$ et $(i, j) \in \Gamma$ avec $\varepsilon(i, j)=1$, on note $\widetilde{\varepsilon}_{i, j} \in \mathfrak{F}_{\Gamma}$ telle que

$$
\widetilde{\varepsilon}_{i, j}(i, j)=-1 \text { et } \widetilde{\varepsilon}_{i, j}(a)=\varepsilon(a) \forall a \in \Gamma \text { avec } a \neq(i, j) .
$$

Pour une partie $\Gamma \subset I \times J$ et $(i, j) \in \Gamma$, on note $\Gamma_{i, j}=\{(a, b) \in \Gamma \mid a \neq i$ et $b \neq j\}$. On obtient le résultat suivant :

LEMme 4.3.3. - On a les égalités et l'inclusion suivantes :

$$
\coprod_{\eta \in \mathfrak{F}_{\Gamma_{i, j}}} \coprod_{\substack{\varepsilon \in \mathfrak{F}_{\Gamma}, \varepsilon(i, j)=\left.1 \\ \varepsilon\right|_{\Gamma_{i, j}}=\eta}} \operatorname{Int}\left(\mathrm{C}_{\varepsilon}^{\mathrm{L}} \cap \mathrm{C}_{\widetilde{\varepsilon}_{i, j}}^{\mathrm{L}}\right)=\mathfrak{h}_{L}^{\Gamma \backslash\{(i, j)\}} \cap\left\{x_{i}=x_{j}\right\},
$$

$$
\begin{aligned}
& \coprod_{\eta \in \mathfrak{F}_{\Gamma_{i, j}}} \operatorname{Int}\left(\mathrm{C}_{\eta}^{\mathrm{L}+[\mathrm{i}, \mathrm{j}]} \cap\left\{\mathrm{u}_{\mathrm{i}}=0\right\}\right) \\
& \coprod_{\substack{\varepsilon \in \mathfrak{F}_{\Gamma}, \varepsilon(i, j)=\left.1 \\
\varepsilon\right|_{\Gamma_{i, j}=\eta}=\eta}} \operatorname{Int}\left(\mathrm{C}_{\varepsilon}^{\Gamma_{i, j}} \cap \mathrm{C}_{\widetilde{\varepsilon}_{\mathrm{i}, \mathrm{j}}}^{\mathrm{L}}\right) \subset \operatorname{Int}\left(\mathrm{C}_{\eta}^{\mathrm{L}+[\mathrm{i}, \mathrm{j}]} \cap\left\{\mathrm{u}_{\mathrm{i}}=0\right\}\right)^{(2)}
\end{aligned}
$$

pour $\eta \in \mathfrak{F}_{\Gamma_{i, j}}$.

Démonstration. - Démontrons le point (1), on a tout d'abord

$$
\mathfrak{h}_{L}^{\Gamma \backslash\{(i, j)\}} \cap\left\{x_{i}=x_{j}\right\}=\coprod_{\alpha \in \mathfrak{F}_{\Gamma \backslash\{(i, j)\}}} \operatorname{Int}\left(\mathrm{C}_{\alpha}^{\mathrm{L}}\right) \cap\left\{x_{i}=x_{j}\right\}
$$

Puis, pour $\alpha \in \mathfrak{F}_{\Gamma \backslash\{(i, j)\}}$, soit $\varepsilon \in \mathfrak{F}_{\Gamma}$ le prolongement de $\alpha$ à $\Gamma$ tel que $\varepsilon(i, j)=1$. On a alors

$$
\operatorname{Int}\left(\mathrm{C}_{\alpha}^{\mathrm{L}}\right) \cap\left\{x_{i}=x_{j}\right\}=\operatorname{Int}\left(\mathrm{C}_{\varepsilon}^{\mathrm{L}} \cap \mathrm{C}_{\widetilde{\varepsilon}_{\mathrm{i}, \mathrm{j}}}^{\mathrm{L}}\right) .
$$

On en déduit l'égalité. La deuxième égalité se montre de la même manière. Pour le point $(3)$, soit $x \in \operatorname{Int}\left(\mathrm{C}_{\varepsilon}^{\mathrm{L}} \cap \mathrm{C}_{\widetilde{\varepsilon}_{\mathrm{i}, \mathrm{j}}}^{\mathrm{L}}\right)$, on a alors

$$
\begin{array}{ll}
\eta(a, b)\left(x_{a}-x_{b}\right)>0 & \forall(a, b) \in \Gamma_{i, j} \\
\varepsilon(a, b)\left(x_{a}-x_{b}\right)>0 & \forall(a, b) \in \Gamma \backslash\left(\Gamma_{i, j} \cup\{(i, j)\}\right)
\end{array}
$$

On en déduit l'inclusion.

Remarque. - Pour $x \in \mathfrak{h}_{L}^{\Gamma \backslash\{(i, j)\}} \cap\left\{x_{i}=x_{j}\right\}$, il existe un unique $\varepsilon \in \mathfrak{F}_{\Gamma}$ tel que $\varepsilon(i, j)=1$ et $x \in \operatorname{Int}\left(\mathrm{C}_{\varepsilon}^{\mathrm{L}} \cap \mathrm{C}_{\widetilde{\varepsilon}_{\mathrm{i}, \mathrm{j}}}^{\mathrm{L}}\right)$.

Pour $\varepsilon \in \mathfrak{F}_{\Gamma}$ tel que $\varepsilon(i, j)=1$ et $w \in \mathcal{E}\left(\mathfrak{h}_{L}, d\right)$, la fonction

$$
\left.x \in C_{\varepsilon}^{L} \cap C_{\widetilde{\varepsilon}_{i, j}}^{L} \longmapsto \partial(w) \theta\right|_{C_{\varepsilon}^{L}}(x)-\left.\partial(w) \theta\right|_{C_{\tilde{\varepsilon}_{i, j}}^{L}}(x)
$$

est définie et continue, on pose la définition suivante :

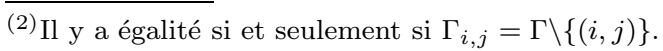


DÉfinition 4.3.4. - Pour $\theta \in \mathcal{W}_{c}\left(\mathfrak{h}_{L}, d, \Gamma\right),(i, j) \in \Gamma$ et $w \in \mathcal{E}\left(\mathfrak{h}_{L}, d\right)$, on considère la fonction définie sur $\mathfrak{h}_{L}^{\Gamma \backslash\{(i, j)\}} \cap\left\{x_{i}=x_{j}\right\}$ par

$$
\langle\partial(w) \theta\rangle_{i, j}(x)=\left.\partial(w) \theta\right|_{C_{\varepsilon}^{L}}(x)-\left.\partial(w) \theta\right|_{C_{\tilde{\varepsilon}_{i, j}}^{L}}(x) .
$$

où $\varepsilon \in \mathfrak{F}_{\Gamma}$ est tel que $\varepsilon(i, j)=1$.

Remarque. - D'après le point (1) de la définition 4.3.2, si on a $s_{i, j}(w)=-w$ alors $\langle\partial(w) \theta\rangle_{i, j}=0$.

Le lemme suivant caractérise l'espace $\mathcal{W}_{c}\left(\mathfrak{h}_{L}, d, \Gamma \backslash\{(i, j)\}\right)$ en tant que sous-espace de $\mathcal{W}_{c}\left(\mathfrak{h}_{L}, d, \Gamma\right)$.

Lemme 4.3.5. - Soit $(i, j) \in \Gamma$ et $\phi \in \mathcal{W}_{c}\left(\mathfrak{h}_{L}, d, \Gamma\right)$ telle que

$$
\langle\partial(w) \phi\rangle_{i, j}=0
$$

pour $w \in \mathcal{E}\left(\mathfrak{h}_{L}, d\right)$. On a alors $\phi \in \mathcal{W}_{c}\left(\mathfrak{h}_{L}, d, \Gamma \backslash\{(i, j)\}\right)$.

Démonstration. - Soit $\eta \in \mathfrak{F}_{\Gamma \backslash\{(i, j)\}}$ et $\varepsilon \in \mathfrak{F}_{\Gamma}$ son prolongement à $\Gamma$ tel que $\varepsilon(i, j)=1$. On a alors

$$
\operatorname{Int}\left(\mathrm{C}_{\varepsilon}^{\mathrm{L}}\right) \coprod \operatorname{Int}\left(\mathrm{C}_{\widetilde{\varepsilon}_{\mathrm{i}, \mathrm{j}}}\right) \subset \operatorname{Int}\left(\mathrm{C}_{\eta}^{\mathrm{L}}\right)
$$

et

$$
C_{\varepsilon}^{L} \cup C_{\tilde{\varepsilon}_{i, j}}^{L}=C_{\eta}^{L}
$$

La fonction $\partial(w) \phi$ se prolonge par continuité sur $C_{\varepsilon}^{L}$ et $C_{\tilde{\varepsilon}_{i, j}}^{L}$. D'après l'hypothèse, ces prolongement coïncident $\operatorname{sur} C_{\varepsilon}^{L} \cap C_{\tilde{\varepsilon}_{i, j}}^{L}$ ainsi $\partial(w) \phi$ se prolonge par continuité sur $C_{\eta}^{L}$ et on en déduit que $\phi \in \mathcal{W}_{c}\left(\mathfrak{h}_{L}, d, \Gamma \backslash\{(i, j)\}\right)$.

Pour un élément $M \in \Omega$ disjoint de $L$ la transformée de Cayley $c_{L}$ induit un isomorphisme d'algèbres de $\operatorname{Sym}\left(\mathfrak{h}_{M, \mathbb{C}}\right)$ dans $\operatorname{Sym}\left(\mathfrak{h}_{M+L, \mathbb{C}}\right)$ qui se restreint en un isomorphisme d'espaces vectoriels entre $\mathcal{E}\left(\mathfrak{h}_{M}, d\right)$ et $\mathcal{E}\left(\mathfrak{h}_{M+L}, d\right)$. Pour $(i, j) \in I \times J$, on note $\alpha_{i, j}=\left(x_{j}-x_{i}\right) / 2$ et $\beta_{i, j}=\left(x_{i}+x_{j}\right) / 2$.

On définit le sous-espace de $\mathcal{W}_{c}\left(\mathfrak{h}_{L}, d, \Gamma\right)$ suivant :

DÉfinition 4.3.6. - Pour $\Gamma \subset I_{L} \times J_{L}$ non vide, on considère l'espace $\widetilde{\mathcal{W}}_{c}\left(\mathfrak{h}_{L}, d, \Gamma\right)$ constitué des fonctions $\phi$ de l'espace $\mathcal{W}_{c}\left(\mathfrak{h}_{L}, d, \Gamma\right)$ qui vérifient les propriétés suivantes :

Pour $(i, j) \in \Gamma$, il existe $\psi_{i, j} \in \widetilde{\mathcal{W}}_{c}\left(\mathfrak{h}_{L+[i, j]}, d, \Gamma_{i, j}\right)$ telle que

$$
\langle\partial(w) \phi\rangle_{i, j}(x)=\mathrm{i} \partial\left(c_{i, j}(w)\right) \psi_{i, j}(x)
$$

pour tout $w \in \mathcal{E}\left(\mathfrak{h}_{L}, d\right)$ et $x \in \mathfrak{h}_{L}^{\Gamma \backslash\{(i, j)\}} \cap\left\{x_{i}=x_{j}\right\}$; si $\Gamma=\varnothing$, on pose $\widetilde{\mathcal{W}}_{c}\left(\mathfrak{h}_{L}, d, \Gamma\right)=$ $\mathcal{W}_{c}\left(\mathfrak{h}_{L}, d, \Gamma\right)$.

\section{REMARQues}

(1) Comme on a $\operatorname{Card}\left(\Gamma_{i, j}\right)<\operatorname{Card}(\Gamma)$, l'espace $\widetilde{\mathcal{W}}_{c}\left(\mathfrak{h}_{L}, d, \Gamma\right)$ est bien défini par récurrence sur le cardinal de $\Gamma$.

(2) On observe que $\Gamma \subset I_{L} \times J_{L}$ implique $\Gamma_{i, j} \subset I_{L+[i, j]} \times J_{L+[i, j]}$. 
(3) D'après le lemme 4.3.3, on a $\mathfrak{h}_{L}^{\Gamma \backslash\{(i, j)\}} \cap\left\{x_{i}=x_{j}\right\} \subset \mathfrak{h}_{L+[i, j]}^{\Gamma_{i, j}} \cap\left\{u_{i}=0\right\}$ ainsi la fonction $\partial\left(c_{i, j}(w)\right) \psi_{i, j}$ est définie sur $\mathfrak{h}_{L}^{\Gamma \backslash\{(i, j)\}} \cap\left\{x_{i}=x_{j}\right\}$.

Pour une partie compacte $\mathcal{K}$ de $\mathfrak{h}_{L}$, on note $\widetilde{\mathcal{W}}_{\mathcal{K}}\left(\mathfrak{h}_{L}, d, \Gamma\right)$ le sous-espace de $\widetilde{\mathcal{W}}_{c}\left(\mathfrak{h}_{L}, d, \Gamma\right)$ des fonctions dont le support est inclus dans $\mathcal{K}$. Sur l'espace $\widetilde{\mathcal{W}}_{\mathcal{K}}\left(\mathfrak{h}_{L}, d, \Gamma\right)$, on considère la topologie induite de $\mathcal{H}_{\mathcal{K}}\left(\mathfrak{h}_{L}, d, \Gamma\right)$. De plus, on considère sur $\widetilde{\mathcal{W}}_{c}\left(\mathfrak{h}_{L}, d, \Gamma\right)$ la topologie de limite inductive $\lim _{\mathcal{K}} \widetilde{\mathcal{W}}_{\mathcal{K}}\left(\mathfrak{h}_{L}, d, \Gamma\right)$. Avec les notations de la définition précédente, on a :

Lemme 4.3.7. - Pour $(i, j) \in \Gamma$, on a l'égalité

$$
\widetilde{\mathcal{W}}_{c}\left(\mathfrak{h}_{L}, d, \Gamma \backslash\{i, j\}\right)=\widetilde{\mathcal{W}}_{c}\left(\mathfrak{h}_{L}, d, \Gamma\right) \cap \mathcal{W}_{c}\left(\mathfrak{h}_{L}, d, \Gamma \backslash\{i, j\}\right)
$$

Démonstration. - On raisonne par récurrence sur le cardinal de $\Gamma$. Si $\Gamma=\{(i, j)\}$, l'égalité à montrer s'écrit

$$
\widetilde{\mathcal{W}}_{c}\left(\mathfrak{h}_{L}, d, \varnothing\right)=\widetilde{\mathcal{W}}_{c}\left(\mathfrak{h}_{L}, d,\{i, j\}\right) \cap \mathcal{W}_{c}\left(\mathfrak{h}_{L}, d, \varnothing\right) .
$$

Comme $\widetilde{\mathcal{W}}_{c}\left(\mathfrak{h}_{L}, d, \varnothing\right)=\mathcal{W}_{c}\left(\mathfrak{h}_{L}, d, \varnothing\right)$, l'égalité (4.3.2) est équivalente à

$$
\mathcal{W}_{c}\left(\mathfrak{h}_{L}, d, \varnothing\right) \subset \widetilde{\mathcal{W}}_{c}\left(\mathfrak{h}_{L}, d,\{(i, j)\}\right)
$$

qui est vérifiée.

On suppose maintenant que l'égalité (4.3.1) est vérifiée pour toute partie $Q \varsubsetneqq \Gamma$.

Montrons tout d'abord l'inclusion $\subset$, soit $\phi \in \widetilde{\mathcal{W}}_{c}\left(\mathfrak{h}_{L}, d, \Gamma \backslash\{(i, j)\}\right)$. Par définition, on a $\phi \in \mathcal{W}_{c}\left(\mathfrak{h}_{L}, d, \Gamma \backslash\{(i, j)\}\right)$ et on remarque que

$$
\mathcal{W}_{c}\left(\mathfrak{h}_{L}, d, \Gamma \backslash\{(i, j)\}\right) \subset \mathcal{W}_{c}\left(\mathfrak{h}_{L}, d, \Gamma\right) .
$$

Pour $(a, b) \in \Gamma \backslash\{(i, j)\}$, il existe $\psi_{a, b} \in \widetilde{\mathcal{W}}_{c}\left(\mathfrak{h}_{L+[a, b]}, d,(\Gamma \backslash\{(i, j)\})_{a, b}\right)$ telle que

$$
\langle\partial(w) \phi\rangle_{a, b}(x)=\mathrm{i} \partial\left(c_{a, b}(w)\right) \psi_{a, b}(x)
$$

pour $x \in \mathfrak{h}_{L}^{\Gamma \backslash\{(i, j),(a, b)\}} \cap\left\{x_{a}=x_{b}\right\}$. On a l'égalité $(\Gamma \backslash\{(i, j)\})_{a, b}=\Gamma_{a, b} \backslash\{(i, j)\}$ ainsi, d'après l'hypothèse de récurrence, on a

$$
\begin{aligned}
\widetilde{\mathcal{W}}_{c}\left(\mathfrak{h}_{L+[a, b]}, d,(\Gamma \backslash\{(i, j)\})_{a, b}\right) & \\
& =\widetilde{\mathcal{W}}_{c}\left(\mathfrak{h}_{L+[a, b]}, d, \Gamma_{a, b}\right) \cap \mathcal{W}_{c}\left(\mathfrak{h}_{L+[a, b]}, d,\left(\Gamma_{a, b}\right) \backslash\{(i, j)\}\right),
\end{aligned}
$$

d'où $\psi_{a, b} \in \widetilde{\mathcal{W}}_{c}\left(\mathfrak{h}_{L+[a, b]}, d, \Gamma_{a, b}\right)$. Maintenant pour $(a, b)=(i, j)$, on a

$$
\langle\partial(w) \phi\rangle_{i, j}=0
$$

pour $w \in \mathcal{E}\left(\mathfrak{h}_{L}, d\right)$. Ainsi, en posant $\psi_{i, j}=0 \in \widetilde{\mathcal{W}}_{c}\left(\mathfrak{h}_{L+[i, j]}, d, \Gamma_{i, j}\right)$, on en déduit que $\phi \in \widetilde{\mathcal{W}}_{c}\left(\mathfrak{h}_{L}, d, \Gamma\right)$. 
Pour l'inclusion $\supset$, soit $\phi \in \widetilde{\mathcal{W}}_{c}\left(\mathfrak{h}_{L}, d, \Gamma\right) \cap \mathcal{W}_{c}\left(\mathfrak{h}_{L}, d, \Gamma \backslash\{(i, j)\}\right)$. Pour $(a, b) \in$ $\Gamma \backslash\{(i, j)\}$, il existe $\psi_{a, b} \in \widetilde{\mathcal{W}}_{c}\left(\mathfrak{h}_{L+[a, b]}, d, \Gamma_{a, b}\right)$ telle que

$$
\langle\partial(w) \phi\rangle_{a, b}(x)=\mathrm{i} \partial\left(c_{a, b}(w)\right) \psi_{a, b}(x)
$$

pour $x \in \mathfrak{h}_{L}^{\Gamma \backslash\{(a, b)\}} \cap\left\{x_{a}=x_{b}\right\}$. Si $(i, j) \notin \Gamma_{a, b}$, alors $(\Gamma \backslash\{(i, j)\})_{a, b}=\Gamma_{a, b}$ ainsi, on a

$$
\psi_{a, b} \in \widetilde{\mathcal{W}}_{c}\left(\mathfrak{h}_{L+[a, b]}, d,(\Gamma \backslash\{(i, j)\})_{a, b}\right) .
$$

Si $(i, j) \in \Gamma_{a, b}$, on a la relation de commutation

$$
\left\langle\langle\partial(w) \phi\rangle_{a, b}\right\rangle_{i, j}=\left\langle\langle\partial(w) \phi\rangle_{i, j}\right\rangle_{a, b}
$$

ainsi, comme $\langle\partial(w) \phi\rangle_{i, j}=0$, on obtient d'après le lemme 4.3 .5 que la fonction $\psi_{a, b}$ appartient à $\mathcal{W}_{c}\left(\mathfrak{h}_{L+[a, b]}, d, \Gamma_{a, b} \backslash\{(i, j)\}\right)$. D'après l'hypothèse de récurrence, on a

$$
\psi_{a, b} \in \widetilde{\mathcal{W}}_{c}\left(\mathfrak{h}_{L+[a, b]}, d,\left(\Gamma_{a, b}\right) \backslash\{(i, j)\}\right) .
$$

On en déduit que $\phi \in \widetilde{\mathcal{W}}_{c}\left(\mathfrak{h}_{L}, d, \Gamma \backslash\{(i, j)\}\right)$.

Lemme 4.3.8. - Pour $\phi \in \widetilde{\mathcal{W}}_{c}\left(\mathfrak{h}_{L}, d, \Gamma\right)$ et $(i, j) \in \Gamma$

(1) Pour $\eta \in \mathfrak{F}_{\Gamma_{i, j}}$, la fonction $\langle\partial(w) \phi\rangle_{i, j}$ se prolonge par continuité sur $C_{\eta}^{L+[i, j]} \cap$ $\left\{u_{i}=0\right\}$ pour $w \in \mathcal{E}\left(\mathfrak{h}_{L}, d\right)$.

(2) Si $\langle\partial(w) \phi\rangle_{i, j}=0$ pour tout $w \in \mathcal{E}\left(\mathfrak{h}_{L}, d\right)$ alors $\phi \in \widetilde{\mathcal{W}}_{c}\left(\mathfrak{h}_{L}, d, \Gamma \backslash\{(i, j)\}\right)$.

Démonstration. - Comme la fonction $\partial\left(c_{i, j}(u)\right) \psi_{i, j}$ se prolonge par continuité sur $C_{\eta}^{L+[i, j]}$, on en déduit le point (1). Pour montrer le point (2), d'après le lemme 4.3.5, on a $\phi \in \mathcal{W}_{c}\left(\mathfrak{h}_{L}, d, \Gamma \backslash\{(i, j)\}\right)$ ainsi,

$$
\phi \in \widetilde{\mathcal{W}}_{c}\left(\mathfrak{h}_{L}, d, \Gamma\right) \cap \mathcal{W}_{c}\left(\mathfrak{h}_{L}, d, \Gamma \backslash\{(i, j)\}\right)
$$

d'où le résultat d'après le lemme 4.3.7.

On fixe une fonction plateau $\eta$ sur $\mathbb{R}$ paire telle $\eta(t)=1$ pour $|t|<1$. Pour $\phi \in$ $\widetilde{\mathcal{W}}_{c}\left(\mathfrak{h}_{L}, d, \Gamma\right)$, il existe $\psi_{i, j} \in \widetilde{\mathcal{W}}_{c}\left(\mathfrak{h}_{L+[i, j]}, d, \Gamma_{i, j}\right)$ telle que

$$
\langle\partial(w) \phi\rangle_{i, j}(x)=\mathrm{i} \partial\left(c_{i, j}(w)\right) \psi_{i, j}(x)
$$

pour $w \in \mathcal{E}\left(\mathfrak{h}_{L}, d\right)$ et $x \in \mathfrak{h}_{L}^{\Gamma \backslash\{(i, j)\}} \cap\left\{x_{i}=x_{j}\right\}$. On note $\rho_{i, j}$ la projection de $\mathfrak{h}_{L}$ sur $\mathfrak{h}_{L} \cap\left\{x_{i}=x_{j}\right\}$ suivant la décomposition

$$
\mathfrak{h}_{L}=\mathfrak{h}_{L} \cap\left\{x_{i}=x_{j}\right\} \oplus \mathbb{R} A_{i, j}
$$

On a alors la définition suivante :

DÉFINITION 4.3.9. - On pose pour $x \in \mathfrak{h}_{L}^{\Gamma_{i, j} \cup\{(i, j)\}}$

$$
\phi_{i, j}(x)=\frac{\operatorname{sign}\left(\alpha_{i, j}\right)}{2} \eta\left(\alpha_{i, j}\right) \sum_{p=0}^{d} \frac{\mathrm{i}^{p+1}\left|\alpha_{i, j}\right|^{p}}{p !} \partial\left(u_{i}^{p}\right) \psi_{i, j}\left(\rho_{i, j}(x)\right) .
$$

La fonction $\phi_{i, j}$ ne dépend que de $\phi$, on dira que $\phi_{i, j}$ est associée à $\phi$ et $(i, j) \in \Gamma$. 


\section{REMARQUeS}

(1) Comme la fonction $\partial\left(u_{i}^{p}\right) \psi_{i, j}$ est définie sur $\mathfrak{h}_{L+[i, j]}^{\Gamma_{i, j}}$ la fonction $\phi_{i, j}$ est bien définie.

(2) La fonction $\eta$ est fixée une fois pour toute.

Proposition 4.3.10. - Pour $\phi \in \widetilde{\mathcal{W}}_{c}\left(\mathfrak{h}_{L}, d, \Gamma\right)$ et $(i, j) \in \Gamma$, la fonction $\phi_{i, j}$ associée $\grave{a} \phi$ et $(i, j)$ appartient $\grave{a} \widetilde{\mathcal{W}}_{c}\left(\mathfrak{h}_{L}, d, \Gamma_{i, j} \cup\{(i, j)\}\right)$ et vérifie

$$
\phi-\phi_{i, j} \in \widetilde{\mathcal{W}}_{c}\left(\mathfrak{h}_{L}, d, \Gamma \backslash\{(i, j)\}\right)
$$

ainsi, on a en particulier

$$
\left\langle\partial(w) \phi_{i, j}\right\rangle_{i, j}=\langle\partial(w) \phi\rangle_{i, j}
$$

pour $w \in \mathcal{E}\left(\mathfrak{h}_{L}, d\right)$.

Démonstration. - Si $\Gamma=\varnothing$, le lemme est évident. On raisonne par récurrence, on suppose que le lemme est vérifié pour toute partie $Q \varsubsetneqq \Gamma$. Montrons que $\phi_{i, j} \in \widetilde{\mathcal{W}}_{c}\left(\mathfrak{h}_{L}, d, \Gamma_{i, j} \cup\{(i, j)\}\right)$. On observe que $\phi_{i, j}$ est une fonction de classe $C^{d}$ sur $\mathfrak{h}_{L}^{\Gamma_{i, j} \cup\{(i, j)\}}$ à support borné. De plus, comme $\partial\left(u_{i}^{p}\right) \psi_{i, j}$ est paire en $u_{q}$ pour $q \in \mathrm{A}_{L} \subset \mathrm{A}_{L+[i, j]}, \phi_{i, j}$ est paire en $u_{q}$ pour $q \in \mathrm{A}_{L}$. On considère $r \in \mathcal{E}\left(\mathfrak{h}_{L}, d\right)$ de la forme $r=s \alpha_{i, j}^{q} \beta_{i, j}^{m}$ où $q+m \leqslant d$ et $s$ est un élément indépendant de $x_{i}$ et $x_{j}$. La dérivée $\partial(r) \phi_{i, j}$ est égale à

$$
\begin{aligned}
& \partial(r) \phi_{i, j}(x) \\
& =\frac{\mathrm{i}}{2} \sum_{p=0}^{d} \operatorname{sign}\left(\alpha_{i, j}\right)^{p+1}\left(\sum_{t=q-p}^{q} \frac{\mathcal{C}_{q}^{t} \eta^{(t)}\left(\alpha_{i, j}\right) \alpha_{i, j}^{p-q+t}}{(p-q+t) !}\right) \partial\left(u_{i}^{p} \beta_{i, j}^{m} s\right) \psi_{i, j}\left(\rho_{i, j}(x)\right) .
\end{aligned}
$$

Soit $\varepsilon \in \mathfrak{F}_{\Gamma_{i, j} \cup\{(i, j)\}}$. On note $\lambda \in \mathfrak{F}_{\Gamma_{i, j}}$ sa restriction à $\Gamma_{i, j}$. Comme la fonction $\partial\left(u_{i}^{p} \beta_{i, j}^{m} s\right) \psi_{i, j}$ se prolonge par continuité sur $C_{\lambda}^{L+[i, j]}$, on en déduit d'après la relation (4.3.4) que $\partial(r) \phi_{i, j}$ se prolonge par continuité sur $C_{\varepsilon}^{L}$. On a ainsi montré que $\phi_{i, j} \in$ $\mathcal{W}_{c}\left(\mathfrak{h}_{L}, d, \Gamma_{i, j} \cup\{(i, j)\}\right)$.

Soit $(a, b) \in \Gamma_{i, j}$. Comme $\psi_{i, j} \in \widetilde{\mathcal{W}}_{c}\left(\mathfrak{h}_{L+[i, j]}, d, \Gamma_{i, j}\right)$, il existe une fonction $\theta_{i, j} \in$ $\widetilde{\mathcal{W}}_{c}\left(\mathfrak{h}_{L+[i, j]+[a, b]}, d, \Gamma_{i, j}, b\right)$ telle que

$$
\left\langle\partial(w) \psi_{i, j}\right\rangle_{a, b}(x)=\mathrm{i} \partial\left(c_{a, b}(w)\right) \theta_{i, j}(x)
$$

pour $w \in \mathcal{E}\left(\mathfrak{h}_{L+[i, j]}, d\right)$ et $x \in \mathfrak{h}_{L+[i, j]}^{\Gamma_{i, j} \backslash\{(a, b)\}}$. On pose

$$
\gamma_{a, b}(x)=\frac{\operatorname{sign}\left(\alpha_{i, j}\right)}{2} \eta\left(\alpha_{i, j}\right) \sum_{p=0}^{d} \frac{\dot{\mathrm{i}}^{p+1}\left|\alpha_{i, j}\right|^{p}}{p !} \partial\left(u_{i}^{p}\right) \theta_{i, j}\left(\rho_{i, j}(x)\right)
$$

pour $x \in \mathfrak{h}_{L+[a, b]}^{\Gamma_{i, j_{a}, b} \cup\{(i, j)\}}$. Montrons que cette fonction appartient à l'espace $\widetilde{\mathcal{W}}_{c}\left(\mathfrak{h}_{L+[a, b]}, d, \Gamma_{a, b}\right)$. En effet, il existe une fonction $\gamma \in \widetilde{\mathcal{W}}_{c}\left(\mathfrak{h}_{L+[a, b]}, d, \Gamma_{a, b}\right)$ telle que

$$
\langle\partial(w) \phi\rangle_{a, b}(x)=\mathrm{i} \partial\left(c_{a, b}(w)\right) \gamma(x)
$$


pour $w \in \mathcal{E}\left(\mathfrak{h}_{L}, d\right)$ et $x \in \mathfrak{h}_{L}^{\Gamma \backslash\{(a, b)\}} \cap\left\{x_{a}=x_{b}\right\}$. On obtient alors avec la relation de commutation

$$
\left\langle\langle\partial(w) \phi\rangle_{a, b}\right\rangle_{i, j}=\left\langle\langle\partial(w) \phi\rangle_{i, j}\right\rangle_{a, b}
$$

l'équation

$$
\langle\partial(w) \gamma\rangle_{i, j}(x)=\mathrm{i} \partial\left(c_{i, j}(w)\right) \theta_{i, j}(x)
$$

pour $w \in \mathcal{E}\left(\mathfrak{h}_{L+[a, b]}, d\right)$ et $x \in \mathfrak{h}_{L+[a, b]}^{\Gamma_{a, b} \backslash\{(i, j)\}} \cap\left\{x_{i}=x_{j}\right\}$. On en déduit que $\gamma_{a, b}$ est la fonction associée à $\gamma$ pour $(a, b)$. Comme $\operatorname{Card}\left(\Gamma_{a, b}\right)<\operatorname{Card}(\Gamma)$, on sait que la fonction $\gamma_{a, b}$ appartient à $\widetilde{\mathcal{W}}_{c}\left(\mathfrak{h}_{L+[a, b]}, d, \Gamma_{a, b}\right)$ d'après l'hypothèse de récurrence.

Il reste à montrer les relations de saut. On considère $w$ de $\mathcal{E}\left(\mathfrak{h}_{L}, d\right)$ de la forme $w=x_{a}^{q} x_{b}^{m} ;$ on a

$$
\begin{aligned}
\left\langle\partial(w) \phi_{i, j}\right\rangle_{a, b}(x) & =\frac{\operatorname{sign}\left(\alpha_{i, j}\right)}{2} \eta\left(\alpha_{i, j}\right) \sum_{p=0}^{d} \frac{\mathrm{i}^{p+1}\left|\alpha_{i, j}\right|^{p}}{p !} \partial\left(u_{i}^{p}\right)\left\langle\partial(w) \psi_{i, j}\right\rangle_{a, b}\left(\rho_{i, j}(x)\right) \\
& =\mathrm{i} \partial\left(c_{a, b}(w)\right) \Gamma_{a, b}(x) .
\end{aligned}
$$

pour $x \in \mathfrak{h}_{L}^{\left(\Gamma_{i, j} \cup\{(i, j)\}\right) \backslash\{(a, b)\}} \cap\left\{x_{a}=x_{b}\right\}$. Si $v \in \mathcal{E}\left(\mathfrak{h}_{L}, d\right)$ est indépendant de $x_{a}$ et $x_{b}$ tel que $v w \in \mathcal{E}\left(\mathfrak{h}_{L}, d\right)$, la relation précédente montre que

$$
\begin{aligned}
\left\langle\partial(v w) \phi_{i, j}\right\rangle_{a, b}(x) & =\partial(v)\left\langle\partial(w) \phi_{i, j}\right\rangle_{a, b}(x) \\
& =\mathrm{i} \partial(v) \partial\left(c_{a, b}(w)\right) \gamma_{a, b}(x) \\
& =\mathrm{i} \partial\left(c_{a, b}(v w)\right) \gamma_{a, b}(x) ;
\end{aligned}
$$

en effet on a $c_{a, b}(v)=v$. On a montré que $\phi_{i, j} \in \widetilde{\mathcal{W}}_{c}\left(\mathfrak{h}_{L}, d, \Gamma_{i, j} \cup\{i, j\}\right)$.

D'après le lemme 4.3.8, il suffit de montrer la relation (4.3.3) pour montrer que $\phi-\phi_{i, j} \in \widetilde{\mathcal{W}}_{c}\left(\mathfrak{h}_{S_{L}}, d, \Gamma \backslash\{(i, j)\}\right)$.

Pour $\left|\alpha_{i, j}\right|<1$, l'expression (4.3.4) se simplifie ainsi

$$
\partial(r) \phi_{i, j}(x)=\frac{\mathrm{i}}{2} \eta\left(\alpha_{i, j}\right) \sum_{p=q}^{d} \operatorname{sign}\left(\alpha_{i, j}\right)^{p+1} \frac{\alpha_{i, j}^{p-q}}{(p-q) !} \partial\left(c_{i, j}\left(\alpha_{i, j}^{p} \beta_{i, j}^{m} s\right)\right) \psi_{i, j}\left(\rho_{i, j}(x)\right) .
$$

Cette expression permet d'obtenir l'égalité suivante

$$
\left\langle\partial(r) \phi_{i, j}\right\rangle_{i, j}(x)=\mathrm{i} \partial\left(c_{i, j}\left(\alpha_{i, j}^{q} \beta_{i, j}^{m} s\right)\right) \psi_{i, j}\left(\rho_{i, j}(x)\right)=\langle\partial(r) \phi\rangle_{i, j}(x) .
$$

Le lemme est démontré.

On note $\Gamma_{M}$ l'ensemble $\left\{(i, M(i)) \mid i \in \mathrm{A}_{M}\right\}$. Pour $\Gamma \subset I \times J$, on note $\Omega(\Gamma)$ l'ensemble des éléments $M$ de $\Omega$ tels que $\Gamma_{M} \subset \Gamma$.

ThÉORÈme 4.3.11. - Pour $L \in \Omega$ et $\Gamma \subset I_{L} \times J_{L}$, on a

$$
\widetilde{\mathcal{W}}_{c}\left(\mathfrak{h}_{L}, d, \Gamma\right)=\sum_{M \in \Omega(\Gamma)} \widetilde{\mathcal{W}}_{c}\left(\mathfrak{h}_{L}, d, \Gamma_{M}\right)
$$


Démonstration. - Cette égalité est claire pour $\Gamma=\varnothing$. Soit $\Gamma \subset I_{L} \times J_{L}$ non vide et supposons l'égalité (4.3.5) vraie pour toute partie $Q$ telle que $Q \varsubsetneqq \Gamma$. Soient $\phi \in$ $\widetilde{\mathcal{W}}_{c}\left(\mathfrak{h}_{L}, d, \Gamma\right)$ et $i_{1} \in I$ tel que $J_{i_{1}}=\left\{j \in J \mid\left(i_{1}, j\right) \in \Gamma\right\}$ vérifie $\operatorname{Card}\left(J_{i_{1}}\right)>1$. Si un tel $i_{1}$ n'existe pas alors il n'y a rien à démontrer en effet, il existe $M \in \Omega(\Gamma)$ tel que $\Gamma_{M}=\Gamma$. Sinon, posons en utilisant les notations de la proposition 4.3 .10

$$
R=\phi-\sum_{j \in J_{i_{1}}} \phi_{i_{1}, j}
$$

On observe que $R \in \widetilde{\mathcal{W}}_{c}\left(\mathfrak{h}_{L}, d, \Gamma^{\prime}\right)$ où $\Gamma^{\prime}=\left\{(a, b) \in \Gamma \mid a \neq i_{1}\right\}$; en effet, on a, d'après la proposition 4.3.10, pour $w \in \mathcal{E}\left(\mathfrak{h}_{L}, d\right)$

$$
\langle\partial(w) \phi\rangle_{i_{1}, j}=\left\langle\partial(w) \phi_{i_{1}, j}\right\rangle_{i_{1}, j} \text { et }\left\langle\partial(w) \phi_{i_{1}, k}\right\rangle_{i_{1}, j}=0
$$

pour $k \neq j$, ainsi

$$
\langle\partial(w) R\rangle_{i_{1}, j}=0
$$

pour $w \in \mathcal{E}\left(\mathfrak{h}_{L}, d\right)$ et $j \in J_{i_{1}}$. On en déduit que $R \in \widetilde{\mathcal{W}}_{c}\left(\mathfrak{h}_{L}, d, \Gamma^{\prime}\right)$ d'après le lemme 4.3.8. D'après ce qui précède, on a la somme

$$
\widetilde{\mathcal{W}}_{c}\left(\mathfrak{h}_{L}, d, \Gamma\right)=\sum_{j \in J_{i_{1}}} \widetilde{\mathcal{W}}_{c}\left(\mathfrak{h}_{L}, d, \Gamma_{i_{1}, j} \cup\left\{\left(i_{1}, j\right)\right\}\right)+\widetilde{\mathcal{W}}_{c}\left(\mathfrak{h}_{L}, d, \Gamma^{\prime}\right) .
$$

De plus, on a la relation

$$
\Omega(\Gamma)=\cup_{j \in J_{i_{1}}} \Omega\left(\Gamma_{i_{1}, j} \cup\left\{\left(i_{1}, j\right)\right\}\right) \cup \Omega\left(\Gamma^{\prime}\right) .
$$

Comme $\operatorname{Card}\left(\Gamma_{i_{1}, j}\right) \cup\left\{\left(i_{1}, j\right)\right\}<\operatorname{Card}(\Gamma)$ et $\operatorname{Card}\left(\Gamma^{\prime}\right)<\operatorname{Card}(\Gamma)$, l'hypothèse de récurrence permet de conclure.

En comparant les propriétés des intégrales invariantes et la définition de l'espace $\widetilde{\mathcal{W}}_{c}\left(\mathfrak{h}_{L}, d, I_{L} \times J_{L}\right)$, on obtient le résultat suivant :

Proposition 4.3.12. - Soit $\psi$ l'intégrale invariante de Harish-Chandra sur $\mathfrak{h}_{L}$ d'une fonction de $\mathcal{D}(\mathfrak{g})$ alors $\psi \in \widetilde{\mathcal{W}}_{c}\left(\mathfrak{h}_{L}, d, I_{L} \times J_{L}\right)$ pour tout $d \in \mathbb{N}$. On souhaite à présent montrer que la décomposition obtenue au théorème 4.3 .11 est continue.

Lemme 4.3.13. - Avec les notations de la définition 4.3.9, l'application

$$
\begin{aligned}
\widetilde{\mathcal{W}}_{c}\left(\mathfrak{h}_{L}, d, \Gamma\right) & \longrightarrow \widetilde{\mathcal{W}}_{c}\left(\mathfrak{h}_{L}, d, \Gamma_{i, j} \cup\{(i, j)\}\right) \\
\phi & \longmapsto \phi_{i, j}
\end{aligned}
$$

est continue.

Démonstration. - On a la relation suivante :

$$
\phi_{i, j}(x)=\frac{\operatorname{sign}\left(\alpha_{i, j}\right)}{2} \eta\left(\alpha_{i, j}\right) \sum_{p=0}^{d} \frac{\left|\alpha_{i, j}\right|^{p}}{p !}\left\langle\partial\left(\alpha_{i, j}^{p}\right) \psi_{i, j}\right\rangle_{i, j}\left(\rho_{i, j}(x)\right) .
$$


Si on fixe un compact $\mathcal{K}$ de $\mathfrak{h}_{L}$, il existe un compact $\mathcal{K}^{\prime}$ tel que pour $\phi \in \widetilde{\mathcal{W}}_{\mathcal{K}}\left(\mathfrak{h}_{L}, d, \Gamma\right)$, on a $\phi_{i, j} \in \widetilde{\mathcal{W}}_{\mathcal{K}^{\prime}}\left(\mathfrak{h}_{L}, d, \Gamma_{i, j} \cup\{(i, j)\}\right)$. On en déduit que pour tout élément $w \in \mathcal{E}\left(\mathfrak{h}_{L}, d\right)$, il existe une constante $C>0$ et une famille d'éléments $w_{1}, \ldots, w_{k}$ de $\mathcal{E}\left(\mathfrak{h}_{L}, d\right)$ tels que

$$
p_{w}\left(\phi_{i, j}\right) \leqslant C \sup _{1 \leqslant m \leqslant k} p_{w_{m}}(\phi) .
$$

Ainsi, l'application

$$
\begin{aligned}
\widetilde{\mathcal{W}}_{\mathcal{K}}\left(\mathfrak{h}_{L}, d, \Gamma\right) & \longrightarrow \widetilde{\mathcal{W}}_{\mathcal{K}^{\prime}}\left(\mathfrak{h}_{L}, d, \Gamma_{i, j} \cup\{(i, j)\}\right) \\
\phi & \longmapsto \phi_{i, j}
\end{aligned}
$$

est continue et on en déduit le résultat.

On considère maintenant l'application linéaire naturelle

$$
\begin{aligned}
u_{\Gamma}: \underset{L \in \Omega(\Gamma)}{\oplus} \widetilde{\mathcal{W}}_{c}\left(\mathfrak{h}_{L}, d, \Gamma_{L}\right) & \longrightarrow \widetilde{\mathcal{W}}_{c}\left(\mathfrak{h}_{L}, d, \Gamma\right) \\
\left(\phi_{L}\right)_{L \in \Omega(\Gamma)} & \longmapsto \sum_{L \in \Omega(\Gamma)} \phi_{L}
\end{aligned}
$$

Cette application est continue. D'après la proposition suivante, elle possède un inverse à droite.

Proposition 4.3.14. - Il existe une application linéaire continue

$$
v_{\Gamma}: \widetilde{\mathcal{W}}_{c}\left(\mathfrak{h}_{L}, d, \Gamma\right) \longrightarrow \underset{L \in \Omega(\Gamma)}{\oplus} \widetilde{\mathcal{W}}_{c}\left(\mathfrak{h}_{L}, d, \Gamma_{L}\right)
$$

telle que

$$
u_{\Gamma} \circ v_{\Gamma}=i d_{\widetilde{\mathcal{W}}_{c}\left(\mathfrak{h}_{L}, d, \Gamma\right)}
$$

Démonstration. - Si $\Gamma=\varnothing$, le résultat est clair. Supposons le résultat vrai pour toute partie $Q \subsetneq \Gamma$; ainsi, il existe une application continue

$$
v_{Q}: \widetilde{\mathcal{W}}_{c}\left(\mathfrak{h}_{L}, d, Q\right) \longrightarrow \underset{L \in \Omega(Q)}{\oplus} \widetilde{\mathcal{W}}_{c}\left(\mathfrak{h}_{L}, d, \Gamma_{L}\right)
$$

telle que $u_{Q} \circ v_{Q}=i d_{\widetilde{\mathcal{W}}_{c}\left(\mathfrak{h}_{L}, d, \Gamma\right)}$. En reprenant les notations de la démonstration de la théorème 4.3.11, on peut supposer qu'il existe $i_{1} \in I$ tel que $\operatorname{Card}\left(J_{i_{1}}\right)>1$ sinon il existe $L \in \Omega(\Gamma)$ tel que $\Gamma=\Gamma_{L}$. L'application

$$
\begin{aligned}
\widetilde{\mathcal{W}}_{c}\left(\mathfrak{h}_{L}, d, \Gamma\right) & \longrightarrow \underset{j \in J_{i_{1}}}{\widetilde{\mathcal{W}}_{c}}\left(\mathfrak{h}_{L}, d, \Gamma_{i_{1}, j} \cup\left\{\left(i_{1}, j\right)\right\}\right) \oplus \widetilde{\mathcal{W}}_{c}\left(\mathfrak{h}_{L}, d, \Gamma^{\prime}\right) \\
\phi & \longmapsto\left(\left(\phi_{i_{1}, j}\right)_{j \in J_{i_{1}}}, \phi-\sum_{j \in J_{i_{1}}} \phi_{i_{1}, j}\right)
\end{aligned}
$$

est définie et continue d'après le lemme 4.3.13. En utilisant (4.3.6) pour $Q=\Gamma_{i_{1}, j} \cup$ $\left\{\left(i_{1}, j\right)\right\}$ et $Q=\Gamma^{\prime}$, on en déduit le résultat. 


\subsection{Décomposition en sous-espaces élémentaires}

On fixe $M$ et $N$ deux éléments de $\Omega$ disjoints. Dans cette section, on montre que $\mathcal{W}_{c}\left(\mathfrak{h}_{M}, d, \Gamma_{N}\right)$ se décompose en un produit tensoriel d'espaces plus simples, Théorème 4.4.4.

On pose $K_{M+N}=I_{M+N} \cup J_{M+N}$.

DÉFINITION 4.4.1. - On considère les sous-espaces de $\mathfrak{h}_{M}$ suivants :

(1) Pour $(i, j) \in \mathrm{A}_{M} \times \operatorname{im}(M)$ tel que $M(i)=j$, on pose $\mathfrak{h}_{M, i, j}=\mathbb{R} P_{i, j} \oplus \mathbb{R} A_{i, j}$.

(2) Pour $(i, j) \in I_{M} \times J_{M}$, on pose $\mathfrak{h}_{M, i, j}=\mathbb{R i} E_{i, i} \oplus \mathbb{R i} E_{j, j}$.

(3) Pour $i \in I_{M+N} \cup J_{M+N}$, on pose $\mathfrak{h}_{M, i}=\mathbb{R i} E_{i, i}$.

On a alors l'égalité suivante :

$$
\mathfrak{h}_{M}=\underset{i \in \mathrm{A}_{M}}{\oplus} \mathfrak{h}_{M, i, M(i)} \underset{i \in \mathrm{A}_{N}}{\oplus} \mathfrak{h}_{M, i, N(i)} \underset{i \in K_{M+N}}{\oplus} \mathfrak{h}_{M, i}
$$

À cette décomposition, on associe les coordonnées

$$
\left(u_{i}\right)_{i \in \mathrm{A}_{M}},\left(v_{i}\right)_{i \in \mathrm{A}_{M}},\left(\alpha_{i, N(i)}\right)_{i \in \mathrm{A}_{N}},\left(\beta_{i, N(i)}\right)_{i \in \mathrm{A}_{N}} \text { et }\left(x_{i}\right)_{i \in K_{M+N}} .
$$

On remarque que les espaces $\mathfrak{h}_{M, i, j}$ (cas 1 et 2) sont des sous-algèbres de Cartan d'une sous-algèbre de Lie de $\mathfrak{g}$ isomorphe à $\mathfrak{u}(1,1)$ ainsi on désigne par $\mathfrak{h}_{M, i, j}^{\text {reg les }}$ éléments réguliers de $\mathfrak{h}_{M, i, j}$ en tant que sous-algèbre de Cartan. On rappelle que $\Gamma_{N}=\left\{(i, N(i)) \in I \times J \mid i \in \mathrm{A}_{N}\right\}$. Pour une partie $Q$ de $\Gamma_{N}$, on pose

$$
\mathfrak{h}_{M}^{Q}=\left\{x \in \mathfrak{h}_{M} \mid x_{i} \neq x_{j} \forall(i, j) \in Q\right\}
$$

On observe que l'on a l'égalité

$$
\mathfrak{h}_{M}^{\Gamma_{N}}=\underset{i \in \mathrm{A}_{M}}{\oplus} \mathfrak{h}_{M, i, M(i)} \underset{i \in \mathrm{A}_{N}}{\bigoplus} \mathfrak{h}_{M, i, N(i)}^{\mathrm{reg}} \underset{i \in K_{M+N}}{\oplus} \mathfrak{h}_{M, i} .
$$

Pour les espaces de la définition 4.4.1, on considère les espaces de fonctions suivants :

DÉfinition 4.4.2. - Pour $(i, j) \in I_{M} \times J_{M}$, on note $\mathcal{W}_{c}\left(\mathfrak{h}_{M, i, j}, d,[i, j]\right) \subset$ $\mathcal{H}\left(\mathfrak{h}_{M, i, j}^{\text {reg }}, d\right)$ constitué des fonctions $\phi$ telles que pour $w \in \mathcal{E}\left(\mathfrak{h}_{M, i, j}, d\right)$ de la forme $w=\alpha_{i, j}^{p} \beta_{i, j}^{q}$, la fonction $\partial(w) \phi$ se prolonge par continuité sur $\mathfrak{h}_{M, i, j}$ si $p$ est impair.

On note aussi $\mathcal{W}_{c}\left(\mathfrak{h}_{M, i, j}, d, \varnothing\right)$ l'espace $\mathcal{C}_{c}^{d}\left(\mathfrak{h}_{M, i, j}\right)$.

Remarque. - On a $\mathcal{W}_{c}\left(\mathfrak{h}_{M, i, j}, d, \varnothing\right) \subset \mathcal{W}_{c}\left(\mathfrak{h}_{M, i, j}, d,[i, j]\right)$

\section{DÉFINITION 4.4.3}

(1) Pour $i \in \mathrm{A}_{M}, j=M(i)$ on note $\mathcal{W}_{c}\left(\mathfrak{h}_{M, i, j}, d\right)$ l'espace des fonctions $\phi$ de classe $C^{d} \operatorname{sur} \mathfrak{h}_{M, i, j}$ à support borné et paires en $u_{i}$.

(2) Pour $i \in I_{M} \cup J_{M}$, on note $\mathcal{W}_{c}\left(\mathfrak{h}_{M, i}, d\right)$ l'espace $\mathcal{C}_{c}^{d}\left(\mathfrak{h}_{M, i}\right)$. 
On désigne par $\mathcal{W}_{c}$ l'un des espaces précédents. Pour une partie compacte $\mathcal{K}$, on note $\mathcal{W}_{\mathcal{K}}$ le sous-espace des fonctions de $\mathcal{W}_{c}$ à support dans $\mathcal{K}$. On considère sur $\mathcal{W}_{\mathcal{K}}$ la topologie fournie par les semi-normes

$$
p_{w}(\phi)=\sup _{x}|\partial(w) \phi(x)|
$$

où $w$ est un élément de degré inférieur à $d$.

Dans cette section, on souhaite démontrer le théorème suivant :

ThÉorème 4.4.4. - Pour $M, N \in \Omega$ disjoints et $d \in \mathbb{N}$, on a

$$
\begin{aligned}
\mathcal{W}_{c}\left(\mathfrak{h}_{M}, d, \Gamma_{N}\right) & \frac{\otimes_{i \in A_{M}} \mathcal{W}_{c}\left(\mathfrak{h}_{M, i, M(i)}, d\right) \underset{i \in \mathrm{A}_{N}}{\otimes} \mathcal{W}_{c}\left(\mathfrak{h}_{M, i, N(i)}, d,[i, N(i)]\right) \underset{i \in K_{M+N}}{\otimes} \mathcal{W}_{c}\left(\mathfrak{h}_{M, i}, d\right)}{\otimes}
\end{aligned}
$$

Pour cela, on a besoin du résultat classique suivant :

Proposition 4.4 .5 ([10, Théorème 39.2]). — Pour E, F deux $\mathbb{R}$-espaces vectoriels de dimension finie, le sous-espace $\mathcal{C}_{c}^{d}(E) \otimes \mathcal{C}_{c}^{d}(F)$ est séquentiellement dense dans $\mathcal{C}_{c}^{d}(E \times F)$.

DÉfinition 4.4.6. - Soit $F$ un $\mathbb{R}$-espace vectoriel de dimension finie et $A$ une partie convexe fermée de $F$ d'intérieur non vide. On note $\mathcal{C}_{c}^{d}(A)$ le sous-espace de $\mathcal{C}^{d}(\operatorname{Int}(\mathrm{A}))$ des fonctions $\phi$ à support borné telles que pour tout $w \in \mathcal{E}(F, d)$, la fonction $\partial(w) \phi$ se prolonge par continuité sur $A$.

Pour une partie compacte $\mathcal{K}$ de $A$, on note $\mathcal{C}_{\mathcal{K}}^{d}(A)$ le sous-espace de $\mathcal{C}_{c}^{d}(A)$ des fonctions à support dans $\mathcal{K}$ et on considère sur cet espace les semi-normes

$$
p_{w}(\phi)=\sup _{x \in A}|\partial(w) \phi(x)| .
$$

On munit l'espace $\mathcal{C}_{c}^{d}(A)$ de la topologie de limite inductive $\lim _{\mathcal{K}} \mathcal{C}_{\mathcal{K}}^{d}(A)$.

Remarque. - Si $A=F$, on retrouve la définition de $\mathcal{C}_{c}^{d}(F)$ précédente.

Grâce au théorème de Whitney sur le prolongement des fonctions, on a la proposition suivante qui doit être bien connue que l'on rappelle faute de référence.

Proposition 4.4.7. - Soit $F$ un $\mathbb{R}$-espace vectoriel de dimension finie et $A$ une partie convexe fermée de $F$ d'intérieur non vide. L'application de restriction

$$
\begin{aligned}
\mathcal{C}_{c}^{d}(F) & \longrightarrow \mathcal{C}_{c}^{d}(A) \\
\phi & \left.\longmapsto \phi\right|_{A}
\end{aligned}
$$

est surjective.

Démonstration. - Soit $\mathcal{K}$ une partie compacte de $A$ et $\phi \in \mathcal{C}_{\mathcal{K}}^{d}(A)$. On considère un voisinage compact $\mathcal{K}^{\prime}$ de $\mathcal{K}$ et $\eta$ une fonction lisse sur $E$ telle $\operatorname{Supp}(\eta) \subset \mathcal{K}^{\prime}$ et $\eta(t)=1$ pour $t \in \mathcal{K}$. On a ainsi $\phi \in \mathcal{C}_{\mathcal{K}^{\prime} \cap A}^{d}(A)$. Soit $\left(e_{i}\right)_{i \in X}$ une base de $E$. Pour $n \in \mathbb{N}^{X}$, on note $|n|=\sum_{i \in X} n_{i}$; pour $x \in E$, on pose $x^{n}=\prod_{i \in X} x_{i}^{n_{i}}$ où $x_{i}$ est 
la $i^{\text {ème }}$ coordonnées de $x$ et $n$ ! représente l'entier $\prod_{i \in X} n_{i}$ !. On désigne par $\phi^{n}$ le prolongement par continuité de

$$
\partial\left(\prod_{i \in X} e_{i}^{n_{i}}\right) \phi
$$

à $\mathcal{K}^{\prime} \cap A$. La famille de fonctions $\left(\phi^{n}\right)_{n \in X}$ constitue une jet d'ordre $d$ sur $\mathcal{K}^{\prime} \cap A$ (cf. [9, p. 68]). D'après la définition de $\phi^{n}$ et comme $\mathcal{K}^{\prime} \cap A$ est convexe, on a pour $n \in \mathbb{N}^{X}$, $r \in N$ tels que $|n| \leqslant r \leqslant d$ et $x, y \in \mathcal{K}^{\prime} \cap A$, le développement de Taylor suivant :

$$
\phi^{n}(y)-\sum_{\substack{m \in \mathbb{N}^{X} \\|m+n| \leqslant d}} \frac{(y-x)^{m}}{m !} \phi^{n+m}(x)=o\left(|y-x|^{r-|n|}\right) .
$$

On en déduit que $\left(\phi^{n}\right)_{n \in \mathbb{N}^{X}}$ est une fonction de Whitney de classe $C^{d}$ sur $\mathcal{K}^{\prime} \cap A$ (cf. [9, Définition 1.6]), ainsi d'après le théorème 2.2 de [9], il existe une fonction $\psi \in \mathcal{C}^{d}(E)$ telle que $\left.\psi\right|_{\mathcal{K}^{\prime} \cap A}=\phi$. On en déduit que la fonction $\psi \eta \in \mathcal{C}_{c}^{d}(E)$ et vérifie $\left.(\psi \eta)\right|_{A}=\phi$.

On obtient la généralisation de la proposition 4.4.5 suivante :

Proposition 4.4.8. - Soient $E$ (resp. $F$ ) un $\mathbb{R}$-espace vectoriel de dimension finie et $A$ (resp. B) une partie convexe fermée d'intérieur non vide de $E$ (resp. $F), \mathcal{C}_{c}^{d}(A) \otimes$ $\mathcal{C}_{c}^{d}(B)$ est séquentiellement dense dans $\mathcal{C}_{c}^{d}(A \times B)$ donc en particulier

$$
\mathcal{C}_{c}^{d}(A \times B)=\overline{\mathcal{C}_{c}^{d}(A) \otimes \mathcal{C}_{c}^{d}(B)}
$$

Démonstration. - On observe tout d'abord que $A \times B$ est un fermé convexe de $E \times F$ d'intérieur non vide. Soit $\phi \in \mathcal{C}_{c}^{d}(A \times B)$. D'après la proposition 4.4.7, il existe une fonction $\psi \in \mathcal{C}_{c}^{d}(E \times F)$ qui prolonge $\phi$. Soit $\mathcal{K}_{1}$ (resp. $\left.\mathcal{K}_{2}\right)$ un compact de $E$ (resp. $F$ ) tels que $\operatorname{Supp}(\psi) \subset \mathcal{K}_{1} \times \mathcal{K}_{2}$. D'après la proposition 4.4 .5 , il existe deux suites $\left(\theta_{n}\right)_{n \geqslant 0}$ de $\mathcal{C}_{\mathcal{K}_{1}}^{d}(E)$ et $\left(\gamma_{n}\right)_{n \geqslant 0}$ de $\mathcal{C}_{\mathcal{K}_{2}}^{d}(F)$ telles que

$$
\left(\theta_{n}, \gamma_{n}\right) \stackrel{n \rightarrow \infty}{\longrightarrow} \psi
$$

dans $\mathcal{C}_{\mathcal{K}_{1} \times \mathcal{K}_{2}}^{d}(E \times F)$. La continuité de l'application $\left.\phi \mapsto \phi\right|_{A}$ permet d'obtenir que

$$
\left(\left.\theta_{n}\right|_{A},\left.\gamma_{n}\right|_{B}\right) \stackrel{n \rightarrow \infty}{\longrightarrow} \phi
$$

dans $\mathcal{C}_{\left(\mathcal{K}_{1} \cap A\right) \times\left(\mathcal{K}_{2} \cap B\right)}^{d}(A \times B)$, d'où le résultat.

Pour une partie $P$ de $I_{M} \times J_{M}$, on rappelle que $\mathfrak{F}_{P}$ désigne l'ensemble des applications de $P$ dans $\{ \pm 1\}$ et on pose pour $\varepsilon \in \mathfrak{F}_{P}$

$$
\mathfrak{h}_{M}^{\varepsilon}=\left\{x \in \mathfrak{h}_{M} \mid \varepsilon(i, j) \alpha_{i, j} \geqslant 0 \forall(i, j) \in P\right\} .
$$


DÉfinition 4.4.9. - Soit $P \subset \Gamma_{N}$. Pour $i \in A_{N}$, on considère les applications linéaires suivantes :

$$
\begin{aligned}
\gamma_{i}: \mathcal{W}_{c}\left(\mathfrak{h}_{M}, d, P\right) & \longrightarrow \mathcal{W}_{c}\left(\mathfrak{h}_{M}, d, P\right) \\
\phi & \longrightarrow x \in \frac{\phi(x)+\phi\left(s_{i, N(i)}(x)\right)}{2}
\end{aligned}
$$

et

$$
\begin{aligned}
\beta_{i}: \mathcal{W}_{c}\left(\mathfrak{h}_{M}, d, P\right) & \longrightarrow \mathcal{W}_{c}\left(\mathfrak{h}_{M}, d, P\right) \\
\phi & \longrightarrow x \in \frac{\phi(x)-\phi\left(s_{i, N(i)}(x)\right)}{2}
\end{aligned}
$$

REmarque. - Ces applications sont bien définies, vérifient $\gamma_{i}+\beta_{i}=i d$ et $\gamma_{i}$ (resp. $\beta_{i}$ ) commute avec $\gamma_{j}$ et $\beta_{j}$ pour $j \in \mathrm{A}_{N} \backslash\{i\}$.

DÉfinition 4.4.10. - Pour $Q \subset \mathrm{A}_{N}$, on pose

$$
\tau_{Q}=\prod_{i \in Q} \gamma_{i} \prod_{i \in \mathrm{A}_{N} \backslash Q} \beta_{i} .
$$

Définition 4.4.11. - Pour $Q \subset \mathrm{A}_{N}$ et $P \subset \Gamma_{N}$, on note $\mathcal{W}_{c}^{Q}\left(\mathfrak{h}_{M}, d, P\right)$ l'ensemble des fonction $\phi$ de $\mathcal{W}_{c}\left(\mathfrak{h}_{M}, d, P\right)$ telles que

$$
\begin{gathered}
\phi=\phi \circ s_{i, N(i)} \text { pour } i \in Q \\
\phi=-\phi \circ s_{i, N(i)} \text { pour } i \in \mathrm{A}_{N} \backslash Q
\end{gathered}
$$

On considère sur cet espace la topologie induite ${ }^{(3)}$.

Pour $i \in \mathrm{A}_{N}$, la réflexion $s_{i, N(i)}$ se restreint en une réflexion de $\mathfrak{h}_{M, i, N(i)}$ que l'on note aussi $s_{i, N(i)}$. On considère pour $\mathcal{W}_{c}\left(\mathfrak{h}_{M, i, N(i)}, d,[i, j]\right)$ les sous-espaces suivants :

DÉfInItion 4.4.12. - On considère $\mathcal{W}_{c}^{\text {paire }}\left(\mathfrak{h}_{M, i, N(i)}, d\right)$ le sous-espace de $\mathcal{C}_{c}^{d}\left(\mathfrak{h}_{M, i, N(i)}\right)$ des fonctions paires en $\alpha_{i, N(i)}$ (i.e. qui vérifie $\phi \circ s_{i, N(i)}=\phi$ ) et $\mathcal{W}_{c}^{\text {imp. }}\left(\mathfrak{h}_{M, i, N(i)}, d,[i, j]\right)$ le sous-espace de $\mathcal{W}_{c}\left(\mathfrak{h}_{M, i, N(i)}, d,[i, j]\right)$ des fonctions impaires en $\alpha_{i, N(i)}$, qui vérifie $\phi \circ s_{i, N(i)}=-\phi$.

Remarque. - On a

$$
\mathcal{W}_{c}^{Q}\left(\mathfrak{h}_{M}, d, P\right)=\left\{\phi \in \mathcal{W}_{c}\left(\mathfrak{h}_{M}, d, P\right) \mid \tau_{Q}(\phi)=\phi\right\}
$$

On a le premier résultat suivant :

Lemme 4.4.13. - Pour $Q \subset \mathrm{A}_{N}$, on a l'égalité

$$
\mathcal{W}_{c}^{Q}\left(\mathfrak{h}_{M}, d, \Gamma_{N}\right)=\mathcal{W}_{c}^{Q}\left(\mathfrak{h}_{M}, d, \Gamma_{N} \backslash Q\right)
$$

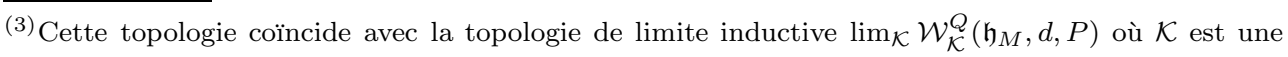
partie compacte de $\mathfrak{h}_{M}$ invariante par les $s_{j, N(j)}, j \in \mathrm{A}_{N}$.
} 
Démonstration. - On a

$$
\mathcal{W}_{c}\left(\mathfrak{h}_{M}, d, \Gamma_{N} \backslash Q\right) \subset \mathcal{W}_{c}\left(\mathfrak{h}_{M}, d, \Gamma_{N}\right),
$$

ainsi, on a l'inclusion

$$
\mathcal{W}_{c}^{Q}\left(\mathfrak{h}_{M}, d, \Gamma_{N} \backslash Q\right) \subset \mathcal{W}_{c}^{Q}\left(\mathfrak{h}_{M}, d, \Gamma_{N}\right) .
$$

Soit $\phi \in \mathcal{W}_{c}^{Q}\left(\mathfrak{h}_{M}, d, \Gamma_{N}\right)$. Pour $i \in Q$, la fonction $\phi$ est paire en $\alpha_{i, N(i)}$ ainsi pour $w \in \mathcal{E}\left(\mathfrak{h}_{M}, d\right)$ tel que $s_{i, N(i)}(w)=w$ la fonction $\partial(w) \phi$ se prolonge par continuité sur $\mathfrak{h}_{M}^{\Gamma_{N} \backslash\{(i, N(i))\}}$. Comme on a déjà ce résultat par définition de $\mathcal{W}_{c}\left(\mathfrak{h}_{M}, d, \Gamma_{N}\right)$ pour $w$ tel que $s_{i, N(i)}(w)=-w$, on en déduit d'après le lemme 4.3.5 que $\phi \in$ $\mathcal{W}_{c}\left(\mathfrak{h}_{M}, d, \Gamma_{N} \backslash\{i, N(i)\}\right)$. En procédant par récurrence sur le cardinal de $Q$, on obtient le résultat.

Les mêmes arguments montrent le résultat suivant :

Lemme 4.4.14. - On a pour $i \in \mathrm{A}_{N}$,

$$
\mathcal{W}_{c}\left(\mathfrak{h}_{M, i, N(i)}, d,[i, N(i)]\right)=\mathcal{W}_{c}^{\text {paire }}\left(\mathfrak{h}_{M, i, N(i)}, d\right) \oplus \mathcal{W}_{c}^{\text {imp. }}\left(\mathfrak{h}_{M, i, N(i)}, d,[i, N(i)]\right) .
$$

Démonstration. - Pour $\phi \in \mathcal{W}_{c}\left(\mathfrak{h}_{M, i, N(i)}, d,[i, N(i)]\right)$, on peut écrire $\phi=\phi_{p}+\phi_{i}$ où $\phi_{p}$ (resp. $\phi_{i}$ ) est une fonction paire (resp. impaire) en $\alpha_{i, N(i)}$. On observe que la fonction $\phi_{i} \in \mathcal{W}_{c}^{\text {imp. }}\left(\mathfrak{h}_{M, i, N(i)}, d,[i, N(i)]\right)$. Montons que $\phi_{p} \in \mathcal{W}_{c}^{\text {paire }}\left(\mathfrak{h}_{M, i, N(i)}, d\right)$. Pour $w=\alpha_{i, N(i)}^{a} \beta_{i, N(i)}^{b} \in \mathcal{E}\left(\mathfrak{h}_{M, i, N(i)}, d\right)$ tel que $a$ est paire; comme $\phi_{p}$ est paire, la fonction $\partial(w) \phi$ se prolonge par continuité sur $\mathfrak{h}_{M, i, N(i)}$. On a aussi ce résultat pour $w$ tel que $a$ est impair par hypothèse ainsi $\phi_{p} \in \mathcal{W}_{c}^{\text {paire }}\left(\mathfrak{h}_{M, i, N(i)}, d\right)$. On en déduit le résultat.

Lemme 4.4.15. - Pour $Q \subset \mathrm{A}_{N}$, l'espace

$$
\underset{i \in \mathrm{A}_{M}}{\otimes} \mathcal{W}_{c}\left(\mathfrak{h}_{M, i, M(i)}, d\right) \underset{i \in Q}{\otimes} \mathcal{W}_{c}^{\text {paire }}\left(\mathfrak{h}_{M, i, N(i)}, d\right) \underset{i \in \mathrm{A}_{N} \backslash Q}{\otimes} \mathcal{W}_{c}^{\text {imp. }}\left(\mathfrak{h}_{M, i, N(i)}, d,[i, j]\right)
$$

est un sous-espace dense de $\mathcal{W}_{c}^{Q}\left(\mathfrak{h}_{M}, d, \Gamma_{N} \backslash Q\right)$.

Démonstration. - On note $\delta$ l'élément de $\mathfrak{F}_{\Gamma_{N} \backslash Q}$ qui prend la valeur 1 pour $(i, j) \in$ $\Gamma_{N} \backslash Q$. On a l'égalité

$$
\mathfrak{h}_{M}^{\delta}=\underset{i \in \mathrm{A}_{M}}{+} \mathfrak{h}_{M, i, M(i)} \underset{i \in Q}{+} \mathfrak{h}_{M, i, N(i)} \underset{i \in \mathrm{A}_{N} \backslash Q}{+} \mathfrak{h}_{M, i, N(i)}^{+} \underset{i \in K_{M+N}}{+} \mathfrak{h}_{M, i}
$$

où

$$
\mathfrak{h}_{M, i, N(i)}^{+}=\left\{x \in \mathfrak{h}_{M, i, N(i)} \mid \alpha_{i, N(i)} \geqslant 0\right\}
$$


ainsi d'après la proposition 4.4 .8 , on a

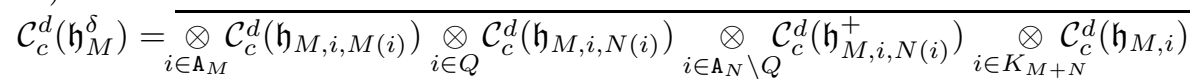

On remarque que $s_{i, N(i)}\left(\mathfrak{h}_{M}^{\delta}\right)=\mathfrak{h}_{M}^{\delta}$ pour $i \in Q$. Notons $\mathcal{C}_{c}^{d, Q}\left(\mathfrak{h}_{M}^{\delta}\right)$ les fonctions $\phi$ de $\mathcal{C}_{c}^{d}\left(\mathfrak{h}_{M}^{\delta}\right)$ telles que $\phi \circ s_{i, N(i)}=\phi$ pour $i \in Q$ (i.e. $\phi$ est paire en $\alpha_{i, N(i)}$ ). De même, on note $\mathcal{C}_{c}^{d, \text { paire }}\left(\mathfrak{h}_{M, i, N(i)}\right)$ les fonctions de $\mathcal{C}_{c}^{d}\left(\mathfrak{h}_{M, i, N(i)}\right)$ paires en $\alpha_{i, N(i)}$ (i.e. qui vérifie $\left.\phi \circ s_{i, N(i)}=\phi\right)$. On déduit de l'égalité (4.4.2)

$$
\begin{aligned}
& \mathcal{C}_{c}^{d, Q}\left(\mathfrak{h}_{M}^{\delta}\right) \\
& =\overline{\otimes_{i \in \mathrm{A}_{M}}^{\otimes} \mathcal{C}_{c}^{d}\left(\mathfrak{h}_{M, i, M(i)}\right) \underset{i \in Q}{\otimes} \mathcal{C}_{c}^{d, \text { paire }}\left(\mathfrak{h}_{M, i, N(i)}\right) \underset{i \in \mathrm{A}_{N} \backslash Q}{\otimes} \mathcal{C}_{c}^{d}\left(\mathfrak{h}_{M, i, N(i)}^{+}\right) \underset{i \in K_{M+N}}{\otimes} \mathcal{C}_{c}^{d}\left(\mathfrak{h}_{M, i}\right)} .
\end{aligned}
$$

On considère l'application

$$
\begin{aligned}
S: \mathcal{W}_{c}^{Q}\left(\mathfrak{h}_{M}, d, \Gamma_{N} \backslash Q\right) & \longrightarrow \mathcal{C}_{c}^{d, Q}\left(\mathfrak{h}_{M}^{\delta}\right) \\
\phi & \left.\longmapsto \phi\right|_{\mathfrak{h}_{M}^{\delta}}
\end{aligned}
$$

On observe que cette application est bien définie. Montrons que c'est un isomorphisme topologique. Pour $i \in \mathrm{A}_{N} \backslash Q$, on a $\phi \circ s_{i, N(i)}=-\phi$ et

$$
s_{i, N(i)}\left(\mathfrak{h}_{M}^{\eta}\right)=\mathfrak{h}_{M}^{\eta^{\prime}}
$$

où

$$
\eta^{\prime}(j)= \begin{cases}\eta(j) & \text { si } j \neq i \\ -\eta(i) & \text { si } i=j\end{cases}
$$

On en déduit que $\left.\phi\right|_{\mathfrak{h}_{M}^{\eta}}=0$ implique $\phi=0$. Soit $\psi \in \mathcal{C}_{c}^{d}\left(\mathfrak{h}_{M}^{\delta}\right)$. Comme

$$
\underset{\eta \in \mathfrak{F}_{\Gamma_{N} \backslash Q}}{\amalg} \operatorname{Int}\left(\mathfrak{h}_{\mathrm{M}}^{\eta}\right)=\mathfrak{h}_{M}^{\Gamma_{N} \backslash Q},
$$

il existe une unique fonction $\phi$ définie sur $\mathfrak{h}_{M}^{\Gamma_{N} \backslash Q}$ qui prolonge $\psi$ et qui vérifie $\phi \circ s_{i, N(i)}=-\phi$ pour $i \in \Gamma_{N} \backslash Q$. On observe alors que $\phi \in \mathcal{W}\left(\mathfrak{h}_{M}, d, \Gamma_{N} \backslash Q\right)$. Par construction $\phi$ est impaire en $\alpha_{i, j}$ pour $(i, j) \in \Gamma_{N} \backslash Q$. Comme $\psi$ est paire en $\alpha_{i, j}$ pour $(i, j) \in Q, \phi$ vérifie la même parité. On en déduit que $\phi \in \mathcal{W}_{c}^{Q}\left(\mathfrak{h}_{M}, d, \Gamma_{N} \backslash Q\right)$. La continuité de l'application est claire. Montrons la continuité de $S^{-1}$. Soit $\mathcal{K}$ une partie compacte de $\mathfrak{h}_{M}^{\delta}$. Soit $\mathcal{K}_{1}$ une partie compacte de $\mathfrak{h}_{M}$ contenant $\mathcal{K}$ telle que $s_{i, N(i)}\left(\mathcal{K}_{1}\right)=\mathcal{K}_{1}$ pour $i \in \Gamma_{N} \backslash Q$ et $\phi \in \mathcal{W}_{\mathcal{K}_{1}}^{Q}\left(\mathfrak{h}_{M}, d, \Gamma_{N} \backslash Q\right)$. L'application $s_{i, N(i)}$ induit un automorphisme d'espace vectoriel de $\mathcal{E}\left(\mathfrak{h}_{M}, d\right)$ que l'on note $s_{i, N(i)}$. Pour $w \in \mathcal{E}\left(\mathfrak{h}_{M}, d\right)$, on a

$$
\sup _{x \in \mathfrak{h}_{M}^{\eta}}|\partial(w) \phi(x)|=\sup _{x \in \mathfrak{h}_{M}^{\eta^{\prime}}}\left|\partial\left(s_{i, N(i)}(w)\right) \phi(x)\right|
$$

où $\eta^{\prime}$ est défini par (4.4). Ainsi, il existe une famille $\left(w_{\eta}\right)_{\eta \in \mathfrak{F}_{\Gamma_{N} \backslash Q}}$ telle que

$$
\sup _{x \in \mathfrak{h}_{M}}|\partial(w) \phi(x)| \leqslant \sup _{\substack{x \in \mathfrak{h}_{M}^{\delta} \\ \eta \in \mathfrak{F}_{\Gamma_{N} \backslash Q}}}\left|\partial\left(w_{\eta}\right) \phi\right|_{\mathfrak{h}_{M}^{\delta}} \mid
$$


On déduit que $S^{-1}$ est continue.

L'image par $S$ de

$$
\underset{i \in A_{M}}{\otimes} \mathcal{W}_{c}\left(\mathfrak{h}_{M, i, M(i)}, d\right) \underset{i \in Q}{\otimes} \mathcal{W}_{c}^{\text {paire }}\left(\mathfrak{h}_{M, i, N(i)}, d\right) \underset{i \in \mathrm{A}_{N} \backslash Q}{\otimes} \mathcal{W}_{c}^{\text {imp. }}\left(\mathfrak{h}_{M, i, N(i)}, d,[i, N(i)]\right)
$$

est égale à

$$
\underset{i \in \mathrm{A}_{M}}{\otimes} \mathcal{C}_{c}^{d}\left(\mathfrak{h}_{M, i, M(i)}\right) \underset{i \in Q}{\otimes} \mathcal{C}_{c}^{d, \text { paire }}\left(\mathfrak{h}_{M, i, N(i)}\right) \underset{i \in \mathrm{A}_{N} \backslash Q}{\otimes} \mathcal{C}_{c}^{d}\left(\mathfrak{h}_{M, i, N(i)}^{+}\right) \underset{i \in K_{M+N}}{\otimes} \mathcal{C}_{c}^{d}\left(\mathfrak{h}_{M, i}\right) .
$$

On en déduit le résultat.

Démonstration du théorème 4.4.4. — On a la somme directe topologique

$$
\mathcal{W}_{c}\left(\mathfrak{h}_{M}, d, \Gamma_{N}\right)=\underset{Q \subset \Gamma_{N}}{\oplus} \mathcal{W}_{c}^{Q}\left(\mathfrak{h}_{M}, d, \Gamma_{N}\right)
$$

Puis, grâce au lemme 4.4.13, on obtient

$$
\mathcal{W}_{c}\left(\mathfrak{h}_{M}, d, \Gamma_{N}\right)=\underset{Q \subset \Gamma_{N}}{\oplus} \mathcal{W}_{c}^{Q}\left(\mathfrak{h}_{M}, d, \Gamma_{N} \backslash Q\right) .
$$

Pour $i \in \mathrm{A}_{N}$, on a

$$
\mathcal{W}_{c}\left(\mathfrak{h}_{M, i, N(i)}, d,[i, N(i)]\right)=\mathcal{W}_{c}^{\text {paire }}\left(\mathfrak{h}_{M, i, N(i)}, d\right) \oplus \mathcal{W}_{c}^{\text {imp. }}\left(\mathfrak{h}_{M, i, N(i)}, d,[i, N(i)]\right)
$$

d'après le lemme 4.4.14. Cette dernière relation permet d'obtenir l'égalité entre

$$
\begin{aligned}
& \underset{Q \subset \Gamma_{N}}{\oplus}\left(\underset { i \in \mathbb { A } _ { M } } { \otimes \mathcal { W } _ { c } } ( \mathfrak { h } _ { M , i , M ( i ) } , d ) \underset { i \in Q } { \otimes \mathcal { W } _ { c } ^ { \text { paire } } } \left(\mathfrak{h}_{M, i, N(i)}, d \underset{i \in \mathbb{A}_{N} \backslash Q}{\otimes \mathcal{W}_{c}^{\text {imp. }}}\left(\mathfrak{h}_{M, i, N(i)}, d,[i, N(i)]\right)\right.\right. \\
& \underset{i \in K_{M+N}}{\left.\otimes \mathcal{W}_{c}\left(\mathfrak{h}_{M, i}, d\right)\right)}
\end{aligned}
$$

et

$$
\underset{i \in A_{M}}{\otimes} \mathcal{W}_{c}\left(\mathfrak{h}_{M, i, M(i)}, d\right) \underset{i \in A_{N}}{\otimes} \mathcal{W}_{c}\left(\mathfrak{h}_{M, i, N(i)}, d,[i, N(i)]\right) \underset{i \in K_{M+N}}{\otimes} \mathcal{W}_{c}\left(\mathfrak{h}_{M, i}, d\right)
$$

On déduit le résultat. 



\section{CHAPITRE 5}

\section{PROPRIÉTÉS DE ChC SI $G$ ET $G^{\prime}$ SONT DE MÊME RANG}

Dans ce chapitre, on étudie sous l'hypothèse que $\mathfrak{g}$ et $\mathfrak{g}^{\prime}$ sont de même rang, les propriétés locales de Chc. On considère des espaces hermitiens $V$ et $V^{\prime}$ de signatures $(\mathrm{p}, \mathrm{q})$ et $(\mathrm{r}, \mathrm{s})$ avec $\mathrm{n}=\mathrm{p}+\mathrm{q}=\mathrm{r}+\mathrm{s}, \mathrm{p} \leqslant \mathrm{q}$ et $\mathrm{r} \leqslant \mathrm{s}$. On conserve les notations introduites dans le chapitre précédent et on pose $I^{\prime}=\{1, \ldots, r\}$ et $J^{\prime}=\{r+1, \ldots, n\}$. On désigne par $\Omega^{\prime}$ l'ensemble des injections d'une partie de $I^{\prime}$ dans $J^{\prime}$. À chaque élément $N$ de $\Omega^{\prime}$, on associe une sous-algèbre de Cartan $\mathfrak{h}_{N}^{\prime}$ de $\mathfrak{g}^{\prime}$ et un système de racines fortement orthogonales $\mathcal{S}$ en utilisant la construction de la sous-section 4.1.1. Pour $N \in \Omega^{\prime}$, on pose pour $\phi \in \mathcal{D}(\mathfrak{g})$ et $x^{\prime} \in \mathfrak{h}_{N}^{\text {/reg }}$

$$
\operatorname{Chc}(\phi)_{N}\left(x^{\prime}\right)=\overline{\pi_{\varnothing}}\left(c_{N}^{-1}\left(x^{\prime}\right)\right) \mathbf{e}_{N}\left(x^{\prime}\right) \operatorname{Chc}(\phi)\left(x^{\prime}\right)
$$

où $\mathbf{e}_{N}\left(x^{\prime}\right)=\operatorname{sign}\left(\prod_{\alpha \in \mathcal{S}} \alpha \circ c_{N}^{-1}\left(x^{\prime}\right)\right)$ et $\mathcal{S}$ représente le système de racines fortement orthogonales associé à $N$. Dans une première section, on étudie les propriétés de $\operatorname{Chc}(\phi)_{\varnothing}$ puis dans la section suivante, on obtient les propriétés de $\operatorname{Chc}(\phi)_{N}$ pour $N \in \Omega^{\prime}$, Théorèmes 5.2 .4 et 5.2.5.

\subsection{Pour une sous-algèbre de Cartan compacte}

Dans cette section, on étudie les propriétés de Chc pour une sous-algèbre de Cartan compacte; les résultats principaux de cette section sont le théorème 5.1.1 (ci-dessous) et le résultat de continuité énoncé dans la proposition 5.1.25.

ThÉORÈme 5.1.1. - Pour $\phi \in \mathcal{D}(\mathfrak{g})$, on a les propriétés

(1) $\operatorname{Chc}(\phi)_{\varnothing}$ est une fonction lisse sur $\mathfrak{h}_{\varnothing}^{\text {'reg }}$.

(2) Pour $w \in \operatorname{Sym}\left(\mathfrak{h}_{\varnothing, \mathbb{C}}^{\prime}\right)$, la fonction $\partial(w) \mathbf{C h c}(\phi)_{\varnothing}$ possède un prolongement par continuité sur l'adhérence de chaque composante connexe de $\mathfrak{h}_{\varnothing}^{\text {'reg }}$.

(3) Les relations de sauts.

REMARQUe. - Le point (3) est explicité au théorème 5.1.30.

On adopte les notations suivantes. On considère des éléments $M$ et $N \in \Omega$ disjoints. On pose $\mathfrak{S}_{\mathrm{n}}=\mathfrak{S}(\{1, \ldots, \mathrm{n}\})$ et on désigne par $L$ un élément de $\mathfrak{S}_{\mathrm{n}}$. On associe 
à $M$ le système de racines fortement orthogonales $\mathcal{S}$ et la sous-algèbre de Cartan $\mathfrak{h}_{M} \in \operatorname{Car}(\mathfrak{g})$. La transformée de Cayley associée à $M$ vérifie

$$
\left(c_{M}^{-1}(x)\right)_{j}= \begin{cases}-\mathrm{i} u_{j}+v_{j} & \text { si } j \in \mathrm{A}_{M}, \\ \mathrm{i} u_{M^{-1}(j)}+v_{M^{-1}(j)} & \text { si } j \in \mathrm{im}(M), \\ x_{j} & \text { sinon. }\end{cases}
$$

On rappelle que

$$
\check{\mathfrak{h}}_{M}=\left\{x \in \mathfrak{h}_{M} \mid u_{i} \leqslant 0 \forall i \in \mathrm{A}_{M}\right\}
$$

et

$$
y_{M L(i)}^{L}= \begin{cases}\operatorname{sign}\left(V_{L(i)}\right) \operatorname{sign}\left(V_{i}^{\prime}\right) & \text { si } L(i) \notin \mathrm{A}_{M} \cup \operatorname{im}(M), \\ 0 & \text { sinon. }\end{cases}
$$

Dans toute cette section $d$ et $d^{\prime}$ sont deux entiers tels que $3 \leqslant 3 d^{\prime} \leqslant d$.

DÉFInItion 5.1.2. - On considère les espaces topologiques suivants pour $d \in \mathbb{N}$

$$
\mathcal{Z}\left(\mathfrak{h}_{\varnothing}^{\prime}, d\right)=\mathcal{H}\left(\mathfrak{h}_{\varnothing}^{\prime \text { reg }}, d\right) \text { et } \mathcal{Z}\left(\mathfrak{h}_{\varnothing}^{\prime}\right)=\mathcal{H}\left(\mathfrak{h}_{\varnothing}^{\prime \text { reg }}, \infty\right)
$$

Pour une partie compacte $\mathcal{K}$ de $\mathfrak{h}_{\varnothing}^{\prime}$, on pose

$$
\mathcal{Z}_{\mathcal{K}}\left(\mathfrak{h}_{\varnothing}^{\prime}, d\right)=\mathcal{H}_{\mathcal{K}}\left(\mathfrak{h}_{\varnothing}^{\prime \text { reg }}, d\right) \text { et } \mathcal{Z}_{\mathcal{K}}\left(\mathfrak{h}_{\varnothing}^{\prime}\right)=\mathcal{H}_{\mathcal{K}}\left(\mathfrak{h}_{\varnothing}^{\prime \text { reg }}, \infty\right)
$$

Pour $\left(i_{0}, j_{0}\right) \in\{1, \ldots, \mathrm{n}\}^{2}$ tel que $i_{0}<j_{0}$, on note $\mathfrak{h}_{\varnothing}^{\prime}{ }^{\left(i_{0}, j_{0}\right)}$ l'espace

$$
\left\{x \in \mathfrak{h}_{\varnothing}^{\prime} \mid x_{i} \neq x_{j} \forall(i, j) \in\{1, \ldots, \mathrm{n}\}^{2}, i<j \text { et }(i, j) \neq\left(i_{0}, j_{0}\right)\right\} \cap\left\{x_{i_{0}}=x_{j_{0}}\right\} .
$$

Pour $\phi \in \mathcal{Z}\left(\mathfrak{h}_{\varnothing}^{\prime}, d\right)$ et $w \in \mathcal{E}\left(\mathfrak{h}_{\varnothing}^{\prime}, d\right)$, on pose pour $x^{\prime} \in \mathfrak{h}_{\varnothing}^{\prime\left(i_{0}, j_{0}\right)}$,

$$
\langle\partial(w) \phi\rangle_{i_{0}, j_{0}}\left(x^{\prime}\right)=\lim _{\substack{t \rightarrow 0 \\ t>0}} \partial(w) \phi\left(x^{\prime}+\mathrm{i} t H\right)-\lim _{\substack{t \rightarrow 0 \\ t>0}} \partial(w) \phi\left(x^{\prime}-\mathrm{i} t H\right)
$$

où $H=E_{i_{0}, i_{0}}-E_{j_{0}, j_{0}}$ représente la coracine de $\alpha=e_{i_{0}}-e_{j_{0}}$.

Remarque. - L'espace $\mathcal{Z}\left(\mathfrak{h}_{\varnothing}^{\prime}, d\right)\left(\right.$ resp. $\left.\mathcal{Z}\left(\mathfrak{h}_{\varnothing}^{\prime}\right)\right)$ est un espace de Fréchet.

On considère aussi des sous-espaces de $\mathfrak{h}_{\varnothing}^{\prime}$ auxquels sont associés des espaces de fonctions $\mathcal{Z}$. Pour $p<q \in\{1, \ldots, \mathrm{n}\}$, on pose

$$
\mathfrak{h}_{\varnothing, p, q}^{\prime}=\mathbb{R i} E_{p, p} \oplus \mathbb{R i} E_{q, q}
$$

Sur $\mathfrak{h}_{\varnothing, p, q}^{\prime}$, on considère l'espace de fonctions suivant :

DÉfinition 5.1.3. - Pour $p, q \in\{1, \ldots, \mathrm{n}\}$, avec $p<q$, on considère l'espace topologique

$$
\mathcal{Z}\left(\mathfrak{h}_{\varnothing, p, q}^{\prime}, d\right)=\mathcal{H}\left(\mathfrak{h}_{\varnothing, p, q}^{\text {reg }}, d\right) .
$$

Pour $w \in \mathcal{E}\left(\mathfrak{h}_{\varnothing, p, q}^{\prime}, d\right)$, on pose pour $x^{\prime} \in \mathfrak{h}_{\varnothing, p, q}^{\prime}$ tel que $x_{p}^{\prime}=x_{q}^{\prime}$

$$
\langle\partial(w) \phi\rangle_{p, q}\left(x^{\prime}\right)=\lim _{\substack{t \rightarrow 0 \\ t>0}} \partial(w) \phi\left(x^{\prime}+\mathrm{i} t H\right)-\lim _{\substack{t \rightarrow 0 \\ t>0}} \partial(w) \phi\left(x^{\prime}-\mathrm{i} t H\right)
$$

où $H=E_{p, p}-E_{q, q}$. 
DÉFInition 5.1.4. - Pour $p \in\{1, \ldots, \mathrm{n}\}$, on note $\mathfrak{h}_{\varnothing, p}^{\prime}=\mathbb{R} i E_{p, p}$ et on considère l'espace topologique $\mathcal{Z}\left(\mathfrak{h}_{\varnothing, p}^{\prime}, d\right)=\mathcal{C}^{d}\left(\mathfrak{h}_{\varnothing}^{\prime}\right)$.

DÉFInition 5.1.5. - Soient $(i, j) \in I_{M} \times J_{M}, p, q$ deux entiers tels que $1 \leqslant p<q \leqslant \mathrm{n}$ et $\phi \in \mathcal{W}_{c}\left(\mathfrak{h}_{M, i, j}, d,[i, j]\right)$. On considère pour $t \in \mathbb{R}_{+}$et $\varepsilon_{1}, \varepsilon_{2}= \pm 1$, la fonction définie $\operatorname{sur} \mathfrak{h}_{\varnothing, p, q}^{\prime \text { reg }}$ par

$$
\mathcal{A}_{i, j}^{p, q, \varepsilon_{1}, \varepsilon_{2}, t}(\phi)\left(x_{p}^{\prime}, x_{q}^{\prime}\right)=\int_{\mathbb{R}^{2}} \frac{\phi\left(x_{i}, x_{j}\right)}{\left(x_{i}+\mathrm{i} t \varepsilon_{1}-x_{p}^{\prime}\right)\left(x_{j}+\mathrm{i} t \varepsilon_{2}-x_{q}^{\prime}\right)} d x_{i} d x_{j}
$$

et on pose

$$
\widetilde{\mathcal{A}}_{i, j}^{p, q, \varepsilon_{1}, \varepsilon_{2}, t}(\phi)\left(x_{p}^{\prime}, x_{q}^{\prime}\right)=\mathcal{A}_{i, j}^{p, q, \varepsilon_{1}, \varepsilon_{2}, t}(\phi)\left(x_{p}^{\prime}, x_{q}^{\prime}\right)-\mathcal{A}_{i, j}^{p, q,-\varepsilon_{2},-\varepsilon_{1}, t}(\phi)\left(x_{q}^{\prime}, x_{p}^{\prime}\right) .
$$

Proposition 5.1.6. - Avec les notations de la définition précédente, on a les propriétés suivantes

(1) Pour $x^{\prime} \in \mathfrak{h}_{\varnothing, p, q}^{\text {reg }}$, la limite

$$
\lim _{\substack{t \rightarrow 0 \\ t>0}} \widetilde{\mathcal{A}}_{i, j}^{p, q, \varepsilon_{1}, \varepsilon_{2}, t}(\phi)\left(x^{\prime}\right)
$$

existe. On note la limite obtenue $\widetilde{\mathcal{A}}_{i, j}^{p, q, \varepsilon_{1}, \varepsilon_{2}}(\phi)\left(x^{\prime}\right)$.

(2) La fonction $\widetilde{\mathcal{A}}_{i, j}^{p, q, \varepsilon_{1}, \varepsilon_{2}}(\phi)$ appartient à $\mathcal{Z}\left(\mathfrak{h}_{\varnothing, p, q}^{\prime}, d^{\prime}\right)$.

(3) Si $\phi \in \mathcal{W}_{c}\left(\mathfrak{h}_{M, i, j}, d, \varnothing\right)$ alors

$$
\left\langle\partial(w) \widetilde{\mathcal{A}}_{i, j}^{p, q, \varepsilon_{1}, \varepsilon_{2}}(\phi)\right\rangle_{p, q}=0
$$

pour $w \in \mathcal{E}\left(\mathfrak{h}_{\varnothing, p, q}^{\prime}, d^{\prime}\right)$.

Démonstration. - Le résultat de la proposition 3.4 .13 est vrai pour $\phi \in$ $\mathcal{W}_{c}\left(\mathfrak{h}_{M, i, j}, d,[i, j]\right)$ et $\varepsilon_{1}= \pm 1$ sous la forme suivante :

(1) La limite

$$
\lim _{\substack{t \rightarrow 0 \\ t>0}} \widetilde{\mathcal{A}}_{i, j}^{p, q, \varepsilon_{1},-1, t}(\phi)\left(x^{\prime}\right)
$$

existe pour tout $x^{\prime} \in \mathfrak{h}_{\varnothing, p, q}^{\text {reg }}$.

(2) Pour $w \in \mathcal{E}\left(\mathfrak{h}_{\varnothing, p, q}^{\prime}, d^{\prime}\right), \partial(w) \widetilde{\mathcal{A}}_{i, j}^{p, q, \varepsilon_{1},-1}(\phi)$ se prolonge par continuité sur l'adhérence de chaque composante connexe de $\mathfrak{h}_{\varnothing, p, q}^{\prime \text { reg }}$.

(3) Si $\phi \in \mathcal{W}_{c}\left(\mathfrak{h}_{M, i, j}, d, \varnothing\right)$ alors

$$
\left\langle\partial(w) \widetilde{\mathcal{A}}_{i, j}^{p, q, \varepsilon_{1},-1}(\phi)\right\rangle_{p, q}=0
$$

pour $w \in \mathcal{E}\left(\mathfrak{h}_{\varnothing, p, q}^{\prime}, d^{\prime}\right)$.

Pour obtenir le résultat avec $\varepsilon_{2}=1$, il suffit de remarquer que l'on a la relation

$$
\widetilde{\mathcal{A}}_{i, j}^{p, q, \varepsilon_{1}, \varepsilon_{2}, t}(\phi)=\overline{\widetilde{\mathcal{A}}_{i, j}^{p, q,-\varepsilon_{1},-\varepsilon_{2}, t}(\bar{\phi})} .
$$

On en déduit le résultat. 
Proposition 5.1.7. — L'application linéaire

$$
\widetilde{\mathcal{A}}_{i, j}^{p, q, \varepsilon_{1}, \varepsilon_{2}}: \mathcal{W}_{c}\left(\mathfrak{h}_{M, i, j}, d,[i, j]\right) \longrightarrow \mathcal{Z}\left(\mathfrak{h}_{\varnothing, p, q}^{\prime}, d^{\prime}\right)
$$

est continue.

Démonstration. - Montrons tout d'abord que pour $t>0, \mathcal{K}$ une partie compacte de $\mathfrak{h}_{M, i, j}$ et $x^{\prime} \in \mathfrak{h}_{\varnothing, p, q}^{\text {reg }}$ la forme linéaire

$$
\begin{aligned}
\mathcal{A}_{i, j}^{p, q, \varepsilon_{1}, \varepsilon_{2}, t}(\cdot)\left(x^{\prime}\right): \mathcal{W}_{\mathcal{K}}\left(\mathfrak{h}_{M, i, j}, d,[i, j]\right) & \longrightarrow \mathbb{C} \\
\phi & \longmapsto \mathcal{A}_{i, j}^{p, q, \varepsilon_{1}, \varepsilon_{2}, t}(\phi)\left(x^{\prime}\right)
\end{aligned}
$$

est continue. En effet, on a

$$
\mathcal{A}_{i, j}^{p, q, \varepsilon_{1}, \varepsilon_{2}, t}(\phi)\left(x^{\prime}\right)=\int_{\mathbb{R}^{\{i, j\}}} \frac{\phi\left(x_{i}, x_{j}\right)}{\left(x_{i}+\mathrm{i} t \varepsilon_{1}-x_{p}^{\prime}\right)\left(x_{j}+\mathrm{i} t \varepsilon_{2}-x_{q}^{\prime}\right)} d x_{i} d x_{j}
$$

ainsi, on obtient l'inégalité

$$
\left|\mathcal{A}_{i, j}^{p, q, \varepsilon_{1}, \varepsilon_{2}, t}(\phi)\left(x^{\prime}\right)\right| \leqslant \frac{1}{t^{2}} p_{0}(\phi) \mu(\mathcal{K})
$$

où $\mu(\mathcal{K})$ représente la mesure de $\mathcal{K}$. On en déduit que l'application linéaire $\widetilde{\mathcal{A}}_{i, j}^{p, q, \varepsilon_{1}, \varepsilon_{2}, t}(\cdot)\left(x^{\prime}\right)$ est continue. Comme cette famille d'applications linéaires converge simplement en $t=0$ d'après la proposition 5.1.6, on obtient que

$$
\begin{aligned}
\mathcal{A}_{i, j}^{p, q, \varepsilon_{1}, \varepsilon_{2}}(\cdot)\left(x^{\prime}\right): \mathcal{W}_{\mathcal{K}}\left(\mathfrak{h}_{M, i, j}, d,[i, j]\right) & \longrightarrow \mathbb{C} \\
\phi & \longmapsto \mathcal{A}_{i, j}^{p, q, \varepsilon_{1}, \varepsilon_{2}}(\phi)\left(x^{\prime}\right)
\end{aligned}
$$

est continue d'après le théorème de Banach-Steinhaus. On considère à présent l'ensemble

$$
G r=\left\{(\phi, \psi) \in \mathcal{W}_{\mathcal{K}}\left(\mathfrak{h}_{M, i, j}, d,[i, j]\right) \times \mathcal{Z}\left(\mathfrak{h}_{\varnothing, p, q}^{\prime}, d^{\prime}\right) \mid \widetilde{\mathcal{A}}_{i, j}^{p, q, \varepsilon_{1}, \varepsilon_{2}}(\phi)=\psi\right\} .
$$

Comme les espaces $\mathcal{W}_{\mathcal{K}}\left(\mathfrak{h}_{M, i, j}, d,[i, j]\right)$ et $\mathcal{Z}\left(\mathfrak{h}_{\varnothing, p, q}^{\prime}, d^{\prime}\right)$ sont des espaces de Fréchet, d'après le théorème du graphe fermé, il suffit de montrer que $G r$ est fermé pour obtenir le résultat. Soit $\left(\phi_{n}\right)_{n \geqslant 0}$ une suite de $\mathcal{W}_{\mathcal{K}}\left(\mathfrak{h}_{M, i, j}, d,[i, j]\right)$ telle que

$$
\lim _{n \rightarrow \infty} \phi_{n}=\phi
$$

dans $\mathcal{W}_{\mathcal{K}}\left(\mathfrak{h}_{M, i, j}, d,[i, j]\right)$ et

$$
\lim _{n \rightarrow \infty} \widetilde{\mathcal{A}}_{i, j}^{p, q, \varepsilon_{1}, \varepsilon_{2}}\left(\phi_{n}\right)=\psi
$$

dans $\mathcal{Z}\left(\mathfrak{h}_{\varnothing, p, q}, d,[i, j]\right)$. Pour $x^{\prime} \in \mathfrak{h}_{\varnothing, p, q}^{\text {reg }}$, d'après (5.1.1) et (5.1.2), on a

$$
\lim _{n \rightarrow \infty} \widetilde{\mathcal{A}}_{i, j}^{p, q, \varepsilon_{1}, \varepsilon_{2}}\left(\phi_{n}\right)\left(x^{\prime}\right)=\widetilde{\mathcal{A}}_{i, j}^{p, q, \varepsilon_{1}, \varepsilon_{2}}(\phi)\left(x^{\prime}\right) .
$$

On a aussi d'après (5.1.3)

$$
\lim _{n \rightarrow \infty} \widetilde{\mathcal{A}}_{i, j}^{p, q, \varepsilon_{1}, \varepsilon_{2}}\left(\phi_{n}\right)\left(x^{\prime}\right)=\psi\left(x^{\prime}\right),
$$

ainsi on obtient l'égalité

et $G r$ est un fermé.

$$
\widetilde{\mathcal{A}}_{i, j}^{p, q, \varepsilon_{1}, \varepsilon_{2}}(\phi)=\psi
$$

MÉMOIRES DE LA SMF 93 
DÉFInition 5.1.8. - Soient $i \in \mathrm{A}_{M}, j=M(i), p, q \in\{1, \ldots, \mathrm{n}\}$ avec $p<q$ et $\phi \in \mathcal{W}_{c}\left(\mathfrak{h}_{M, i, j}, d, \varnothing\right)$. On considère la fonction définie sur $\mathfrak{h}_{\varnothing, p, q}^{\prime \text { reg }}$ par

$$
\mathcal{B}_{i, j}^{p, q}(\phi)\left(x_{p}^{\prime}, x_{q}^{\prime}\right)=2 \int_{u_{i} \leqslant 0, v_{i} \in \mathbb{R}} \frac{\phi\left(u_{i}, v_{i}\right)}{\left(-\mathrm{i} u_{i}+v_{i}-x_{p}^{\prime}\right)\left(\mathrm{i} u_{i}+v_{i}-x_{q}^{\prime}\right)} d u_{i} d v_{i}
$$

et on pose $\widetilde{\mathcal{B}}_{i, j}^{p, q}(\phi)\left(x_{p}^{\prime}, x_{q}^{\prime}\right)=\mathcal{B}_{i, j}^{p, q}(\phi)\left(x_{p}^{\prime}, x_{q}^{\prime}\right)-\mathcal{B}_{i, j}^{p, q}(\phi)\left(x_{q}^{\prime}, x_{p}^{\prime}\right)$.

Proposition 5.1.9. - Avec les notations de la définition précédente, la fonction $\widetilde{\mathcal{B}}_{i, j}^{p, q}(\phi)$ appartient à $\mathcal{Z}\left(\mathfrak{h}_{\varnothing, p, q}^{\prime}, d^{\prime}\right)$.

Démonstration. - Le résultat de la proposition 3.4 .4 est vrai pour $\phi \in$ $\widetilde{\mathcal{W}}_{c}\left(\mathfrak{h}_{M, i, j}, d, \varnothing\right)$ sous la forme suivante. Il existe une fonction $D \in \mathcal{C}^{d^{\prime}}\left(\mathfrak{h}_{\varnothing, p, q}^{\prime}\right)$ antisymétrique telle que

$$
\widetilde{\mathcal{B}}_{i, j}^{p, q}(\phi)\left(x_{p}^{\prime}, x_{q}^{\prime}\right)=-2 \mathbf{i} \pi^{2} \operatorname{sign}\left(x_{q}^{\prime}-x_{p}^{\prime}\right) \phi\left(\underset{u_{i}}{0}, \underset{v_{i}}{\min }\left(x_{p}^{\prime}, x_{q}^{\prime}\right)\right)+D\left(x_{p}^{\prime}, x_{q}^{\prime}\right) .
$$

On en déduit le résultat.

Proposition 5.1.10. - L'application linéaire

$$
\widetilde{\mathcal{B}}_{i, j}^{p, q}: \mathcal{W}_{c}\left(\mathfrak{h}_{M, i, j}, d, \varnothing\right) \longrightarrow \mathcal{Z}\left(\mathfrak{h}_{\varnothing, p, q}^{\prime}, d^{\prime}\right)
$$

est continue.

Démonstration. - Pour $t>0$, on considère l'application linéaire

$$
\mathcal{B}_{i, j}^{p, q, t}(\phi)\left(x_{p}^{\prime}, x_{q}^{\prime}\right)=\int_{u_{i} \leqslant-t, v_{i} \in \mathbb{R}} \frac{\phi\left(u_{i}, v_{i}\right)}{\left(-\mathrm{i} u_{i}+v_{i}-x_{p}^{\prime}\right)\left(\mathrm{i} u_{i}+v_{i}-x_{q}^{\prime}\right)} d u_{i} d v_{i} .
$$

Pour $x^{\prime} \in \mathfrak{h}_{\varnothing, p, q}^{\text {reg }}$ et $\mathcal{K}$ une partie compacte de $\mathfrak{h}_{M, i, j}$, on observe que

$$
\left|\mathcal{B}_{i, j}^{p, q, t}(\phi)\left(x^{\prime}\right)\right| \leqslant \frac{1}{t^{2}} p_{0}(\phi) \mu(\mathcal{K})
$$

où $\mu(\mathcal{K})$ représente la mesure de $\mathcal{K}$. On en déduit que l'application

$$
\begin{aligned}
\mathcal{B}_{i, j}^{p, q, t}(\cdot)\left(x^{\prime}\right): \mathcal{W}_{\mathcal{K}}\left(\mathfrak{h}_{M, i, j}, d, \varnothing\right) & \longrightarrow \mathbb{C} \\
\phi & \longmapsto \mathcal{B}_{i, j}^{p, q, t}(\phi)\left(x^{\prime}\right)
\end{aligned}
$$

est continue. Comme $\mathcal{B}_{i, j}^{p, q, t}(\phi)\left(x^{\prime}\right)$ converge vers $\mathcal{B}_{i, j}^{p, q}(\phi)\left(x^{\prime}\right)$ en $t=0$, on obtient d'après le théorème de Banach-Steinhaus que

$$
\begin{aligned}
\mathcal{B}_{i, j}^{p, q}(\cdot)\left(x^{\prime}\right): \mathcal{W}_{\mathcal{K}}\left(\mathfrak{h}_{M, i, j}, d, \varnothing\right) & \longrightarrow \mathbb{C} \\
\phi & \longmapsto \mathcal{B}_{i, j}^{p, q}(\phi)\left(x^{\prime}\right)
\end{aligned}
$$

est continue. On considère ensuite l'ensemble

$$
G r=\left\{(\phi, \psi) \in \mathcal{W}_{\mathcal{K}}\left(\mathfrak{h}_{M, i, j}, d, \varnothing\right) \times \mathcal{Z}\left(\mathfrak{h}_{\varnothing, p, q}^{\prime}, d^{\prime}\right) \mid \widetilde{\mathcal{B}}_{i, j}^{p, q}(\phi)=\psi\right\} .
$$

Comme les espaces $\mathcal{W}_{\mathcal{K}}\left(\mathfrak{h}_{M, i, j}, d, \varnothing\right)$ et $\mathcal{Z}\left(\mathfrak{h}_{\varnothing, p, q}^{\prime}, d^{\prime}\right)$ sont des espaces de Fréchet, d'après le théorème du graphe fermé, il suffit de montrer que $G r$ est fermé pour obtenir le résultat. Soit $\left(\phi_{n}\right)_{n \geqslant 0}$ une suite de $\mathcal{W}_{\mathcal{K}}\left(\mathfrak{h}_{M, i, j}, d, \varnothing\right)$ telle que

$$
\lim _{n \rightarrow \infty} \phi_{n}=\phi
$$


dans $\mathcal{W}_{\mathcal{K}}\left(\mathfrak{h}_{M, i, j}, d, \varnothing\right)$ et

$$
\lim _{n \rightarrow \infty} \widetilde{\mathcal{B}}_{i, j}^{p, q}\left(\phi_{n}\right)=\psi
$$

dans $\mathcal{Z}\left(\mathfrak{h}_{\varnothing, p, q}, d,[i, j]\right)$. Pour $x^{\prime} \in \mathfrak{h}_{\varnothing, p, q}^{\text {reg }}$, d'après (5.1.4) et (5.1.5), on a

$$
\lim _{n \rightarrow \infty} \widetilde{\mathcal{B}}_{i, j}^{p, q}\left(\phi_{n}\right)\left(x^{\prime}\right)=\widetilde{\mathcal{B}}_{i, j}^{p, q}(\phi)\left(x^{\prime}\right) .
$$

On a aussi d'après (5.1.6)

$$
\lim _{n \rightarrow \infty} \widetilde{\mathcal{B}}_{i, j}^{p, q}\left(\phi_{n}\right)\left(x^{\prime}\right)=\psi\left(x^{\prime}\right)
$$

ainsi on obtient l'égalité

$$
\widetilde{\mathcal{B}}_{i, j}^{p, q}(\phi)=\psi
$$

et $G r$ est un fermé.

Pour $(i, j) \in I_{M} \times J_{M}$ et $p, q \in\{1, \ldots, \mathrm{n}\}$ tels que $p<q$, on pose $t r_{p, q}^{i, j}$ l'isomorphisme d'espaces vectoriels entre $\mathfrak{h}_{\varnothing, p, q, \mathbb{C}}^{\prime}$ et $\mathfrak{h}_{M, i, j, \mathbb{C}}$ défini par

$$
\operatorname{tr}_{p, q}^{i, j}\left(E_{p, p}\right)=E_{i, i} \text { et } \operatorname{tr}_{p, q}^{i, j}\left(E_{q, q}\right)=E_{j, j} .
$$

On observe que cette application induit un isomorphisme d'algèbres entre $\operatorname{Sym}\left(\mathfrak{h}_{\varnothing, p, q, \mathbb{C}}^{\prime}\right)$ et $\operatorname{Sym}\left(\mathfrak{h}_{M, i, j, \mathbb{C}}\right)$ qui se restreint en un morphisme d'espaces vectoriels de $\mathcal{E}\left(\mathfrak{h}_{\varnothing, p, q}^{\prime}, d^{\prime}\right)$ sur $\mathcal{E}\left(\mathfrak{h}_{M, i, j}, d\right)$. On a le résultat suivant avec $\widetilde{\mathcal{A}}$ et $\widetilde{\mathcal{B}}$.

Proposition 5.1.11. - On considère une fonction $\phi \in \widetilde{\mathcal{W}}_{c}\left(\mathfrak{h}_{M, i, j}, d,[i, j]\right)$ et une fonction $\psi \in \mathcal{W}_{c}\left(\mathfrak{h}_{M+[i, j], i, j}, d, \varnothing\right)$ telles que

$$
\langle\partial(w) \phi\rangle_{i, j}=\mathrm{i} \partial\left(c_{i, j}(w)\right) \psi
$$

pour $w \in \mathcal{E}\left(\mathfrak{h}_{M, i, j}, d\right)(c f$. Définition 4.3.6). On a alors pour $p, q \in\{1, \ldots, \mathrm{n}\}$ tels que $p<q$ et $w \in \mathcal{E}\left(\mathfrak{h}_{\varnothing, p, q}^{\prime}, d^{\prime}\right)$

$$
\left\langle\partial(w) \widetilde{\mathcal{B}}_{i, j}^{p, q}(\psi)+\partial(w) \widetilde{\mathcal{A}}_{i, j}^{p, q, \varepsilon_{1}, \varepsilon_{2}}(\phi)\right\rangle_{p, q}=2\left|\varepsilon_{1}+\varepsilon_{2}\right| \pi^{2}\left\langle\partial\left(\operatorname{tr}_{p, q}^{i, j}(w)\right) \phi\right\rangle_{i, j} \circ t r_{p, q}^{i, j} .
$$

Démonstration. — D'après la proposition 3.4.14, on a

$$
\left\langle\partial(w) \widetilde{\mathcal{B}}_{i, j}^{p, q}(\psi)+\partial(w) \widetilde{\mathcal{A}}_{i, j}^{p, q,-\varepsilon,-1}(\phi)\right\rangle_{p, q}=2 \pi^{2}(1+\varepsilon)\left\langle\partial\left(\operatorname{tr}_{p, q}^{i, j}(w)\right) \phi\right\rangle_{i, j} \circ t r_{p, q}^{i, j}
$$

pour $w \in \mathcal{E}\left(\mathfrak{h}_{\varnothing, p, q}^{\prime}, d^{\prime}\right)$. On a ensuite les relations

$$
\overline{\widetilde{\mathcal{B}}_{i, j}^{p, q}(\bar{\psi})}=-\widetilde{\mathcal{B}}_{i, j}^{p, q}(\psi), \quad \overline{\widetilde{\mathcal{A}}_{i, j}^{p, q, \varepsilon, 1}(\bar{\phi})}=\widetilde{\mathcal{A}}_{i, j}^{p, q,-\varepsilon,-1}(\phi) .
$$

On note $\bar{w}$ le conjugué de $w \in \operatorname{Sym}\left(\mathfrak{h}_{\varnothing, p, q, \mathbb{C}}^{\prime}\right)$. On obtient

$$
\begin{aligned}
\left\langle\partial(w) \widetilde{\mathcal{B}}_{i, j}^{p, q}(\psi)+\partial(w) \widetilde{\mathcal{A}}_{i, j}^{p, q, \varepsilon, 1}(\phi)\right\rangle_{p, q} & =\overline{\left\langle\partial(\bar{w}) \widetilde{\mathcal{B}}_{i, j}^{p, q}(-\bar{\psi})+\partial(\bar{w}) \widetilde{\mathcal{A}}_{i, j}^{p, q,-\varepsilon,-1}(\bar{\phi})\right\rangle_{p, q}} \\
& =\overline{2 \pi^{2}(1+\varepsilon)\left\langle\partial\left(\operatorname{tr}_{p, q}^{i, j}(\bar{w})\right) \bar{\phi}\right\rangle_{i, j} \circ \operatorname{tr}_{p, q}^{i, j}} \\
& =2 \pi^{2}(1+\varepsilon)\left\langle\partial\left(\operatorname{tr}_{p, q}^{i, j}(w)\right) \phi\right\rangle_{i, j} \circ \operatorname{tr}_{p, q}^{i, j}
\end{aligned}
$$

On en déduit le résultat. 
DÉfinition 5.1.12. - Soit $j \in I_{M} \cup J_{M}, \phi \in \mathcal{W}_{c}\left(\mathfrak{h}_{M, j}, d\right), t \in \mathbb{R}_{+}, \varepsilon= \pm 1$ et $p \in\{1, \ldots, \mathrm{n}\}$, on considère la fonction définie sur $\mathfrak{h}_{\varnothing, p}^{\prime}$ par

$$
\mathcal{C}_{j}^{p, \varepsilon, t}(\phi)\left(x_{p}^{\prime}\right)=\int_{\mathbb{R}} \frac{\phi\left(x_{j}\right)}{x_{j}+\mathrm{i} t \varepsilon-x_{p}^{\prime}} d x_{j} .
$$

Proposition 5.1.13. - Avec les notations de la définition précédente, la limite

$$
\lim _{\substack{t \rightarrow 0 \\ t>0}} \mathcal{C}_{j}^{p, \varepsilon, t}(\phi)
$$

existe; on note la fonction obtenue $\mathcal{C}_{j}^{p, \varepsilon}(\phi)$. Cette fonction appartient à $\mathcal{Z}\left(\mathfrak{h}_{\varnothing, p}^{\prime}, d^{\prime}\right)$.

Démonstration. - Pour $t>0$, on a

$$
\int_{\mathbb{R}} \frac{\phi\left(x_{j}\right)}{x_{j}+\mathrm{i} \varepsilon-x_{p}^{\prime}} d x_{j}=-\int_{\mathbb{R}} \ln \left(x_{j}+\mathrm{i} t \varepsilon-x_{p}^{\prime}\right) \phi^{\prime}\left(x_{j}\right) d x_{j}
$$

On en déduit que

$$
\lim _{t \rightarrow 0} \mathcal{C}_{j}^{p, \varepsilon, t}(\phi)\left(x_{p}^{\prime}\right)
$$

existe et on a

$$
\mathcal{C}_{j}^{p, \varepsilon}(\phi)\left(x_{p}^{\prime}\right)=-\int_{\mathbb{R}} \ln \left|x_{j}-x_{p}^{\prime}\right| \phi^{\prime}\left(x_{j}\right) d x_{j}-\mathrm{i} \varepsilon \pi \phi\left(x_{p}^{\prime}\right) .
$$

On observe alors que la fonction $\mathcal{C}_{j}^{p, \varepsilon}(\phi)$ appartient à $\mathcal{Z}\left(\mathfrak{h}_{\varnothing, p}^{\prime}, d-1\right)$ donc en particulier $\mathcal{Z}\left(\mathfrak{h}_{\varnothing, p}^{\prime}, d^{\prime}\right)$.

Proposition 5.1.14. - L'application

$$
\mathcal{C}_{j}^{p, \varepsilon}: \mathcal{W}_{c}\left(\mathfrak{h}_{M, i}, d\right) \longrightarrow \mathcal{Z}\left(\mathfrak{h}_{\varnothing, p}^{\prime}, d^{\prime}\right)
$$

est continue.

Démonstration. - Soit $\mathcal{K}$ (resp. $\left.\mathcal{K}^{\prime}\right)$ une partie compacte de $\mathfrak{h}_{M, j}$ (resp. $\mathfrak{h}_{\varnothing, p}^{\prime}$ ), une fonction $\phi \in \mathcal{W}_{\mathcal{K}}\left(\mathfrak{h}_{M, j}, d\right)$ et $r \leqslant d^{\prime}$. On a alors

$$
\mathcal{C}_{j}^{p, \varepsilon}(\phi)^{(r)}\left(x_{p}^{\prime}\right)=-\int_{\mathbb{R}} \ln \left|x_{j}-x_{p}^{\prime}\right| \phi^{(r+1)}\left(x_{j}\right) d x_{j}-\mathrm{i} \varepsilon \pi \phi^{(r)}\left(x_{p}^{\prime}\right) .
$$

On en déduit les inégalités

$$
\begin{aligned}
\sup _{x_{p}^{\prime} \in \mathcal{K}^{\prime}} & \left|\mathcal{C}_{j}^{p, \varepsilon}(\phi)^{(r)}\left(x_{p}^{\prime}\right)\right| \\
& \leqslant \sup _{x_{p}^{\prime} \in \mathcal{K}^{\prime}} \int_{\mathcal{K}-x_{p}^{\prime}}|\ln | x_{j}|| d x_{j} \sup _{x_{j} \in \mathfrak{h}_{M, j}}\left|\phi^{(r+1)}\left(x_{j}\right)\right|+\pi \sup _{x_{j} \in \mathfrak{h}_{M, j}}\left|\phi^{(r)}\left(x_{j}\right)\right| \\
& \leqslant \int_{\mathcal{K}-\mathcal{K}^{\prime}}|\ln | x_{j}|| d x_{j} \sup _{x_{j} \in \mathfrak{h}_{M, j}}\left|\phi^{(r+1)}\left(x_{j}\right)\right|+\pi \sup _{x_{j} \in \mathfrak{h}_{M, j}}\left|\phi^{(r)}\left(x_{j}\right)\right|
\end{aligned}
$$

qui permet de conclure. 
On considère les parties de $\{1, \ldots, \mathrm{n}\}$ suivantes :

$$
\begin{aligned}
I_{M, L} & =\left\{i \in\{1, \ldots, \mathrm{n}\} \mid L(i) \in \mathrm{A}_{M}\right\} & J_{M, L} & =\{i \in\{1, \ldots, \mathrm{n}\} \mid L(i) \in \operatorname{im}(M)\} \\
I_{N, L} & =\left\{i \in\{1, \ldots, \mathrm{n}\} \mid L(i) \in \mathrm{A}_{N}\right\} & J_{N, L} & =\{i \in\{1, \ldots, \mathrm{n}\} \mid L(i) \in \operatorname{im}(N)\} \\
I_{M, N, L} & =L^{-1}\left(I_{M+N}\right) & J_{M, N, L} & =L^{-1}\left(J_{M+N}\right) .
\end{aligned}
$$

D'après les définitions de $I_{M, L}$ et $J_{M, L}$ (resp. $I_{N, L}$ et $J_{N, L}$ ), l'application $L^{-1} \circ M \circ L$ (resp. $L^{-1} \circ N \circ L$ ) définit une bijection de $I_{M, L}$ dans $J_{M, L}$ (resp. de $I_{N, L}$ dans $J_{N, L}$ ) que l'on note $\widehat{M}_{L}\left(\right.$ resp. $\left.\widehat{N}_{L}\right)$.

Pour une bijection $L \in \mathfrak{S}_{\mathrm{n}}$, on note $\mathfrak{S}_{M, N, L}$ le sous-groupe du groupe symétrique $\mathfrak{S}_{\mathrm{n}}$ engendré par les transpositions $\left(i, \widehat{M}_{L}(i)\right)_{i \in I_{M, L}}$ et $\left(i, \widehat{N}_{L}(i)\right)_{i \in I_{N, L}}$. On considère alors sur l'ensemble $\mathfrak{S}_{\mathrm{n}}$ la relation suivante :

$$
L \simeq_{M, N} L^{\prime} \text { si il existe } \sigma \in \mathfrak{S}_{M, N, L} \text { tel que } L^{\prime}=L \sigma .
$$

LeMme 5.1.15. - La relation $\simeq_{M, N}$ est une relation d'équivalence.

Démonstration. - Il suffit pour cela de montrer que $L \simeq_{M, N} L^{\prime}$ entraine $\mathfrak{S}_{M, N, L}=$ $\mathfrak{S}_{M, N, L^{\prime}}$. On observe tout d'abord que

$$
\sigma\left(I_{M, L^{\prime}}\right)=I_{M, L} \text { et } \sigma\left(I_{N, L^{\prime}}\right)=I_{N, L} .
$$

Puis, pour $i \in I_{M, L^{\prime}}$, on a

$$
L^{\prime} \circ \widehat{M}_{L^{\prime}}(i)=M \circ L^{\prime}(i)
$$

ainsi, comme $L^{\prime}=L \circ \sigma$, on obtient

$$
L \circ \sigma \circ \widehat{M}_{L^{\prime}} \circ \sigma^{-1}(i)=M \circ L(i)
$$

pour $i \in I_{M, L}$. On en déduit que

$$
\sigma \circ \widehat{M}_{L}^{\prime} \circ \sigma^{-1}=\widehat{M}_{L} .
$$

De même, on a

$$
\sigma \circ \widehat{N}_{L}^{\prime} \circ \sigma^{-1}=\widehat{N}_{L}
$$

Comme $\sigma \in \mathfrak{S}_{M, N, L}$, on en déduit l'égalité

$$
\mathfrak{S}_{M, N, L}=\mathfrak{S}_{M, N, L^{\prime}}
$$

On note $\bar{L}_{M, N}$ la classe de $L$. Chaque classe possède un unique élément $L$ qui vérifie

$$
i<\widehat{N}_{L}(i) \forall i \in I_{N, L} \text { et } i<\widehat{M}_{L}(i) \forall i \in I_{M, L}
$$

que l'on appelle l'élément principal de la classe. On note l'ensemble des éléments principaux de $\simeq_{M, N}$ par $\operatorname{Pr}(M, N)$. On rappelle que $\varepsilon(L)$ désigne la signature de $L$ pour $L \in \mathfrak{S}_{\mathrm{n}}$. 
DÉfinition 5.1.16. - Pour $\phi \in \mathcal{W}_{c}\left(\mathfrak{h}_{M}, d, I_{M} \times J_{M}\right)$, on pose pour $x^{\prime} \in \mathfrak{h}_{\varnothing}^{\text {'reg }}$ et $t>0$ :

$$
\mathcal{R}_{M}^{t}(\phi)\left(x^{\prime}\right)=\sum_{L:\{1, \ldots, \mathrm{n}\} \hookrightarrow\{1, \ldots, \mathrm{n}\}} \varepsilon(L) \int_{\check{\mathfrak{h}}_{M}} \frac{\phi(x)}{\prod_{j \in\{1, \ldots, \mathrm{n}\}}\left(c_{M}^{-1}(x)_{L(j)}+\mathrm{i} t y_{M L(j)}^{L}-x_{j}^{\prime}\right)} d x,
$$

Si $N \in \Omega$ est disjoint de $M$, on pose pour $\phi \in \mathcal{W}_{c}\left(\mathfrak{h}_{M}, d, \Gamma_{N}\right)$

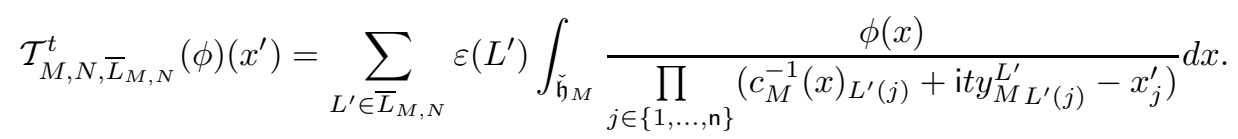

On montre tout d'abord un lemme de continuité ponctuelle.

Lemme 5.1.17. - Pour $\phi \in \mathcal{W}_{c}\left(\mathfrak{h}_{M}, d, \Gamma_{N}\right), N \in \Omega$ tel que $\Gamma_{N} \subset I_{M} \times J_{M}$ et $x^{\prime} \in \mathfrak{h}_{\varnothing}^{\text {reg }}$ la limite

$$
\lim _{\substack{t \rightarrow 0 \\ t>0}} \mathcal{T}_{M, N, \bar{L}_{M, N}}^{t}(\phi)\left(x^{\prime}\right)
$$

existe; on note cette limite $\mathcal{T}_{M, N, \bar{L}_{M, N}}(\phi)\left(x^{\prime}\right)$. De plus, la forme linéaire

$$
\begin{aligned}
\mathcal{W}_{c}\left(\mathfrak{h}_{M}, d, \Gamma_{N}\right) & \longrightarrow \mathbb{C} \\
\phi & \longmapsto \mathcal{T}_{M, N, \bar{L}_{M, N}}(\phi)\left(x^{\prime}\right)
\end{aligned}
$$

est continue.

Démonstration. - En utilisant les arguments utilisés au chapitre III (p.56), on montre que la limite

$$
\lim _{t \rightarrow 0} \mathcal{T}_{M, N, \bar{L}_{M, N}}^{t}(\phi)\left(x^{\prime}\right)
$$

existe pour $x^{\prime} \in \mathfrak{h}_{\varnothing}^{\text {reg }}$. Montrons maintenant la continuité de l'application $\phi \mapsto$ $\mathcal{T}_{M, N, \bar{L}_{M, N}}(\phi)\left(x^{\prime}\right)$. Soit $\mathcal{K}$ une partie compacte de $\mathfrak{h}_{M}$ et $\phi \in \mathcal{W}_{\mathcal{K}}\left(\mathfrak{h}_{M}, d, \Gamma_{N}\right)$. Pour $t>0$, on a

$$
\begin{aligned}
& \left|\int_{\check{\mathfrak{h}}_{M}} \frac{\phi(x)}{\prod_{i \in\{1, \ldots, \mathrm{n}\}}\left(c_{M}^{-1}(x)_{L^{\prime}(i)}+\mathrm{i} t y_{\left.M_{L^{\prime}(i)}^{L^{\prime}}-x_{i}^{\prime}\right)}\right.} d x\right| \\
& \quad \leqslant \mu(\mathcal{K}) \int_{\mathcal{K}}\left|\frac{1}{\prod_{i \in\{1, \ldots, \mathrm{n}\}}\left(c_{M}^{-1}(x)_{L^{\prime}(i)}+\mathrm{i} t y_{M_{L^{\prime}(i)}^{L^{\prime}}}-x_{i}^{\prime}\right)}\right| d x \sup _{x \in \mathfrak{h}_{M}}|\phi(x)|
\end{aligned}
$$

On en déduit que pour $t>0$, l'application

$$
\begin{aligned}
\mathcal{W}_{c}\left(\mathfrak{h}_{M}, d, \Gamma_{N}\right) & \longrightarrow \mathbb{C} \\
\phi & \longmapsto \mathcal{T}_{M, N, \bar{L}_{M, N}^{t}}^{t}(\phi)\left(x^{\prime}\right)
\end{aligned}
$$

est continue. Le théorème de Banach-Steinhaus permet de conclure. 
Proposition 5.1.18. - Pour $\phi \in \mathcal{W}_{c}\left(\mathfrak{h}_{M}, d, \Gamma_{N}\right), N \in \Omega$ tel que $\Gamma_{N} \subset I_{M} \times J_{M}^{(1)}$, on a les propriétés suivantes :

(1) La limite $\lim _{\substack{t \rightarrow 0 \\ t>0}} \mathcal{R}_{M}^{t}(\phi)\left(x^{\prime}\right)$ existe pour $x^{\prime} \in \mathfrak{h}_{\varnothing}^{\text {'reg }}$ et définit une fonction notée $\mathcal{R}_{M}(\phi)$ qui appartient à $\mathcal{Z}\left(\mathfrak{h}_{\varnothing}^{\prime}, d^{\prime}\right)$.

(2) L'application

$$
\mathcal{R}_{M}: \mathcal{W}_{c}\left(\mathfrak{h}_{M}, d, \Gamma_{N}\right) \longrightarrow \mathcal{Z}\left(\mathfrak{h}_{\varnothing}^{\prime}, d^{\prime}\right)
$$

est continue.

Pour démontrer cette proposition, on a besoin de nouvelles définitions. On fixe $M, N \in \Omega$ disjoints et $L$. On pose $K_{M, N, L}=I_{M, N, L} \cup J_{M, N, L}$. Soient $P \subset I_{N, L}$, $Q \subset I_{M, L}$ et $R \subset K_{M, N, L}$. On considère l'espace vectoriel suivant :

$$
\begin{aligned}
& \mathfrak{h}_{P, Q, R}=\underset{i \in P}{\oplus} \mathfrak{h}_{M, L(i), N \circ L(i)} \underset{i \in I_{N, L} \backslash P}{\oplus} \mathfrak{h}^{\prime} \varnothing, i, \widehat{N}_{L}(i) \underset{i \in Q}{\oplus} \mathfrak{h}_{M, L(i), M \circ L(i)} \\
& \underset{i \in I_{M, L} \backslash Q}{\oplus} \mathfrak{h}_{\varnothing, i, \widehat{M}_{L}(i)} \underset{i \in R}{\oplus} \mathfrak{h}_{M, L(i)} \underset{i \in K_{M, N, L} \backslash R}{\oplus} \mathfrak{h}_{\varnothing, i}^{\prime} .
\end{aligned}
$$

Pour $i \in P$ la réflexion $s_{L(i), N \circ L(i)}$ définie sur $\mathfrak{h}_{M, L(i), N \circ L(i)}$ s'étend en une réflexion de $\mathfrak{h}_{P, Q, R}$ suivant la décomposition ci-dessus que l'on note $s_{L(i), N \circ L(i)}$. Cette application induit un automorphisme de $\operatorname{Sym}\left(\mathfrak{h}_{P, Q, R, \mathbb{C}}\right)$. Sur l'espace vectoriel $\mathfrak{h}_{P, Q, R}$, on a les coordonnées

$$
\begin{gathered}
\left(x_{L(i)}\right)_{i \in P},\left(x_{N \circ L(i)}\right)_{i \in P},\left(x_{i}^{\prime}\right)_{i \in I_{N, L} \backslash P},\left(x_{\widehat{N}_{L}(i)}^{\prime}\right)_{i \in I_{N, L} \backslash P},\left(u_{i}\right)_{i \in Q},\left(v_{i}\right)_{i \in Q} \\
\left(x_{i}^{\prime}\right)_{i \in I_{M, L} \backslash Q},\left(x_{\widehat{M}_{L}(i)}^{\prime}\right)_{i \in I_{M, L} \backslash Q}\left(x_{L(i)}\right)_{i \in R},\left(x_{i}^{\prime}\right)_{i \in K_{M, N, L} \backslash R} .
\end{gathered}
$$

On considère l'ouvert de $\mathfrak{h}_{P, Q, R}$

$$
\begin{aligned}
& \mathcal{O}^{P, Q, R}=\underset{i \in P}{\oplus} \mathfrak{h}_{M, L(i), N \circ L(i)}^{\mathrm{reg}} \underset{i \in I_{N, L} \backslash P}{\oplus} \mathfrak{h}_{\varnothing, i, \widehat{N}_{L}(i)}^{\operatorname{reg}} \underset{i \in Q}{\oplus} \mathfrak{h}_{M, L(i), M \circ L(i)} \\
& \underset{i \in I_{M, L} \backslash Q}{\oplus} \mathfrak{h}_{\varnothing, i, \widehat{M}_{L}(i)}^{\prime \text { reg }} \underset{i \in R}{\oplus} \mathfrak{h}_{M, L(i)} \underset{i \in K_{M, N, L} \backslash R}{\oplus} \mathfrak{h}_{\varnothing, i}^{\prime} .
\end{aligned}
$$

DÉFINITION 5.1.19

(1) Une partie $A$ de $\mathfrak{h}_{P, Q, R}$ est dite décomposable si il existe des parties $\mathcal{K}_{i}$ pour $i \in I_{N, L} \cup I_{N, L} \cup K_{M, N, L}$ telles que

$$
A=\underset{i \in I_{N, L} \cup J_{N, L} \cup K_{M, N, L}}{\times} \mathcal{K}_{i} .
$$

suivant la décomposition (5.1.7). Pour $i \in I_{N, L} \cup I_{M, L} \cup K_{M, N, L}$, on appelle $\mathcal{K}_{i}$ la $i$ ème composante de $A$ que l'on note $A_{i}$.

(2) Une partie $A$ de $\mathfrak{h}_{P, Q, R}$ décomposable est dite adaptée si il existe des parties compactes $\mathcal{K}_{i}$ pour $i \in P \cup Q \cup R$ respectivement de $\mathfrak{h}_{M, L(i), N \circ L(i)}, \mathfrak{h}_{M, L(i), M \circ L(i)}$ et $\mathfrak{h}_{M, L(i)}$ telles que

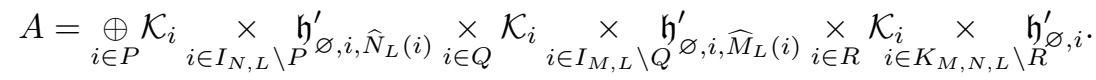

${ }^{(1)}$ Cette hypothèse équivaut à $M$ et $N$ disjoints. 
Notations. - Pour une fonction $\phi \in \mathcal{H}\left(\mathcal{O}^{P, Q, R}, d\right)$, on considère les propriétés suivantes :

(1) Pour $i \in Q$, on dit que $\phi$ vérifie la propriété $\left(P_{i}\right)$ si $\phi$ est paire en $u_{i}$.

(2) Pour $i \in \mathrm{A}_{N}$ et $j=N(i)$, on dit que $\phi$ vérifie la propriété $\left(S_{i, j}\right)$ si $\langle\partial(w) \phi\rangle_{i, j}=0$ dès que $s_{i, j}(w)=-w$.

DÉfinition 5.1.20. - Soient $S \subset P \subset I_{N, L}, T \subset Q \subset I_{M, L}$ et $R \subset K_{M, N, L}$. Sur l'ouvert $\mathcal{O}^{P, Q, R}$, on considère le sous-espace $\mathfrak{F}(P, Q, R, S, T, d)$ de $\mathcal{H}\left(\mathcal{O}^{P, Q}, R, d\right)$ constitué des fonctions $\phi$ à support dans une partie adaptée de $\mathfrak{h}_{P, Q, R}$ qui vérifient $\left(P_{i}\right)$ pour $i \in T$ et $\left(S_{i, j}\right)$ pour $i \in L(S)$ et $j=N(i)$. Pour une partie adaptée $A$ de $\mathfrak{h}_{P, Q, R}$, on note $\mathfrak{F}_{A}(P, Q, R, S, T)$ le sous-espace des fonctions à support dans $A$ et on considère sur cet espace la topologie définie par les seminormes

$$
p_{\mathcal{K}, w}(\phi)=\sup _{x \in \mathcal{K}}|\partial(w) \phi(x)|
$$

où $\mathcal{K}$ est une partie compacte de $A$ et $w \in \operatorname{Sym}\left(\mathfrak{h}_{P, Q, R, \mathbb{C}}\right)$ de degré inférieur à $d$. On considère sur $\mathfrak{F}(P, Q, R, S, T, d)$ la topologie inductive $\lim _{A} \mathfrak{F}_{A}(P, Q, R, S, T, d)$.

On pose pour $i \in P$ et $j \in Q$

$$
\begin{aligned}
\mathfrak{F}(P, Q, R, d) & =\mathfrak{F}(P, Q, R, P, Q, d), \\
\mathfrak{F}_{i}(P, Q, R, d) & =\mathfrak{F}(P, Q, R,\{i\}, \varnothing, d), \\
\mathfrak{F}_{j}(P, Q, R, d) & =\mathfrak{F}(P, Q, R, \varnothing,\{j\}, d), \\
\text { et } \mathfrak{F}_{\varnothing}(P, Q, R, d) & =\mathfrak{F}(P, Q, R, \varnothing, \varnothing, d)
\end{aligned}
$$

Si $A$ est une partie adapté de $\mathfrak{h}_{P, Q, R}$, on observe que $\mathfrak{F}_{A}(P, Q, R)$ est un espace de Fréchet et que l'on a les égalités d'espaces topologiques suivantes

$$
\mathfrak{F}\left(I_{N, L}, J_{N, L}, K_{M, N, L}, d\right)=\mathcal{W}_{c}\left(\mathfrak{h}_{M}, d, \Gamma_{N}\right) \text { et } \mathfrak{F}(\varnothing, \varnothing, \varnothing, d)=\mathcal{Z}\left(\mathfrak{h}_{\varnothing}^{\prime}, d\right)
$$

Remarque. - On a les inclusions d'espaces topologiques

$$
\begin{aligned}
& \mathfrak{F}(P, Q, R, d) \hookrightarrow \mathfrak{F}_{i}(P, Q, R, d), \\
& \mathfrak{F}(P, Q, R, d) \hookrightarrow \mathfrak{F}_{j}(P, Q, R, d), \\
& \mathfrak{F}(P, Q, R, d) \hookrightarrow \mathfrak{F}_{\varnothing}(P, Q, R, d) .
\end{aligned}
$$

Pour $i \in P$, on souhaite étendre l'application $\tilde{\mathcal{A}}_{L(i), N \circ L(i)}^{\tilde{N}_{L(i)},-\operatorname{sign}\left(V_{i}^{\prime}\right), \operatorname{sign}\left(V_{\widehat{N}_{L}(i)}^{\prime}\right)}$ à $\mathfrak{F}(P, Q, R, d)$. Soit $x \in \mathcal{O}^{P, Q, R}$. On écrit alors $x=x_{1}+x_{2}$ où $x_{1} \in \mathfrak{h}_{M, L(i), N \circ L(i)}^{\text {reg }}$ et $x_{2}$ appartient au complémentaire de $\mathfrak{h}_{M, L(i), N \circ L(i)}$ suivant la décomposition (5.1.7). On note $\phi\left(.+x_{2}\right)$ l'application définie sur $\mathfrak{h}_{M, L(i), N \circ L(i)}^{\text {reg }}$ par

$$
\phi\left(.+x_{2}\right)(a)=\phi\left(a+x_{2}\right) .
$$

On observe que $\phi\left(.+x_{2}\right)$ appartient à $\mathcal{W}_{c}\left(\mathfrak{h}_{M, L(i), N \circ L(i)}, d,[L(i), N \circ L(i)]\right)$, ainsi

$$
x \longmapsto \widetilde{\mathcal{A}}_{L(i), N \circ L(i)}^{i, \widehat{N}_{L}(i),-\operatorname{sign}\left(V_{i}^{\prime}\right), \operatorname{sign}\left(V_{\widehat{N}_{L}(i)}^{\prime}\right)}\left(\phi\left(.+x_{2}\right)\right)\left(x_{1}\right)
$$


est une fonction définie sur $\mathcal{O}^{P \backslash\{i\}, Q, R}$. On note cette fonction

$$
\widetilde{\mathcal{A}}_{L(i), N \circ L(i)}^{i, \widehat{N}_{L}(i),-\operatorname{sign}\left(V_{i}^{\prime}\right), \operatorname{sign}\left(V_{\widehat{N}_{L}(i)}^{\prime}\right)}(\phi) .
$$

Lemme 5.1.21. - Pour $i \in P$ et $\phi \in \mathfrak{F}(P, Q, R, d)$, on a

$$
\widetilde{\mathcal{A}}_{L(i), N \circ L(i)}^{i, \widehat{N}_{L}(i),-\operatorname{sign}\left(V_{i}^{\prime}\right), \operatorname{sign}\left(V_{\widehat{N}_{L}(i)}^{\prime}\right)}(\phi) \in \mathfrak{F}\left(P \backslash\{i\}, Q, R, d^{\prime}\right)
$$

et l'application linéaire

$$
\widetilde{\mathcal{A}}_{L(i), N \circ L(i)}^{i, \widehat{N}_{L}(i),-\operatorname{sign}\left(V_{i}^{\prime}\right), \operatorname{sign}\left(V_{\widehat{N}_{L}(i)}^{\prime}\right)}(\phi): \mathfrak{F}(P, Q, R, d) \longrightarrow \mathfrak{F}\left(P \backslash\{i\}, Q, R, d^{\prime}\right)
$$

est continue.

Démonstration. - Soient $A$ une partie adaptée de $\mathfrak{h}(P, Q, R)$ et $B$ la partie adaptée de $\mathfrak{h}(P \backslash\{i\}, Q, R)$ telle $B_{j}=A_{j}$ pour $j \neq i$. On considère $\phi \in \mathfrak{F}_{i, A}(P, Q, R, d)$. Montrons que $\widetilde{\mathcal{A}}_{L(i), N \circ L(i)}^{i, \widehat{N}_{L}(i),-\operatorname{sign}\left(V_{i}^{\prime}\right), \operatorname{sign}\left(V_{\widehat{N}_{L}(i)}^{\prime}\right)}(\phi)$ appartient à $\mathfrak{F}_{\varnothing, B}(P \backslash\{i\}, Q, R, d)$. On observe tout d'abord que

$$
\operatorname{Supp}\left(\widetilde{\mathcal{A}}_{L(i), N \circ L(i)}^{i, \widehat{N}_{L}(i),-\operatorname{sign}\left(V_{i}^{\prime}\right), \operatorname{sign}\left(V_{\widehat{N}_{L}(i)}^{\prime}\right)}(\phi)\right) \subset B
$$

Soit $w=w_{1} w_{2} \in \operatorname{Sym}\left(\mathfrak{h}_{P \backslash\{i\}, Q, R}\right)$ de degré inférieur à $d^{\prime}$ avec $w_{1} \in \operatorname{Sym}\left(\mathfrak{h}_{\varnothing, i, \widehat{N}_{L}(i), \mathbb{C}}\right)$ et $w_{2}$ indépendant de $x_{i}$ et $x_{\widehat{N}_{L}(i)}$. Soit $\mathcal{K}$ une partie compacte de $\mathfrak{h}_{\varnothing, i, \widehat{N}_{L}(i)}^{\prime}$. D'après le lemme 5.1.7, il existe une famille $\left(v_{j}\right)_{1 \leqslant j \leqslant m}$ de $\operatorname{Sym}\left(\mathfrak{h}_{M, L(i), N \circ L(i), \mathbb{C})}\right)$ de degré au plus $\max \left(3,3 \operatorname{deg}\left(w_{1}\right)\right)$ telle que

$$
\sup _{x \in \mathcal{K}}\left|\partial\left(w_{1}\right) \tilde{\mathcal{A}}_{L(i), N \circ L(i)}^{i, \widehat{N}_{L}(i),-\operatorname{sign}\left(V_{i}^{\prime}\right), \operatorname{sign}\left(V_{\widehat{N}_{L}(i)}^{\prime}\right)}(\psi)(x)\right| \leqslant \sup _{1 \leqslant j \leqslant m, x \in A_{i}}\left|\partial\left(v_{j}\right) \psi(x)\right|
$$

pour tout $\psi \in \mathcal{W}_{c}\left(\mathfrak{h}_{M, L(i), N \circ L(i)}, d,[L(i), N \circ L(i)]\right)$. On observe ensuite que

$$
\phi\left(.+x_{2}\right) \in \mathcal{W}_{c}\left(\mathfrak{h}_{M, L(i), N \circ L(i)}, d,[L(i), N \circ L(i)]\right) .
$$

En considérant l'inégalité (5.1.8) pour $\psi=\phi\left(.+x_{2}\right)$, on obtient que

$$
\partial\left(w_{1}\right) \widetilde{\mathcal{A}}_{L(i), N \circ L(i)}^{i, \widehat{N}_{L}(i),-\operatorname{sign}\left(V_{i}^{\prime}\right), \operatorname{sign}\left(V_{\widehat{N}_{L}(i)}^{\prime}\right)}(\psi)
$$

se prolonge par continuité sur l'adhérence des composantes connexes de $\mathcal{O}^{P, Q, R}$ dans $\mathfrak{h}_{P, Q, R}$. Supposons que $\operatorname{deg}\left(w_{2}\right)>0$. Soit $v \neq 0$ appartenant au complémentaire de $\mathfrak{h}_{M, L(i), N \circ L(i)}$ suivant la décomposition (5.1.7). Comme

$$
\frac{\phi\left(.+x_{2}+t v\right)-\phi\left(.+x_{2}\right)}{t}-\partial(v) \phi\left(.+x_{2}\right) \stackrel{t \rightarrow 0}{\longmapsto} 0
$$

dans $\mathcal{W}_{c}\left(\mathfrak{h}_{M, L(i), N \circ L(i)}, d,[L(i), N \circ L(i)]\right)$. On en déduit que

$$
\partial\left(v w_{1}\right) \widetilde{\mathcal{A}}_{L(i), N \circ L(i)}^{i, \widehat{N}_{L}(i),-\operatorname{sign}\left(V_{i}^{\prime}\right), \operatorname{sign}\left(V_{\widehat{N}_{L}(i)}^{\prime}\right)}(\phi)(x)
$$

MÉMOIRES DE LA SMF 93 
existe pour $x \in \mathcal{O}^{P, Q, R}$ de plus, on a l'égalité

$$
\begin{aligned}
\partial\left(v w_{1}\right) \mathcal{\mathcal { A }}_{L(i), N \circ L(i)}^{i, \widehat{N}_{L}(i),-\operatorname{sign}\left(V_{i}^{\prime}\right), \operatorname{sign}\left(V_{\widehat{N}_{L}(i)}^{\prime}\right)}(\phi)(x) & \\
= & \partial\left(w_{1}\right) \widetilde{\mathcal{A}}_{L(i), N \circ L(i)}^{i, \widehat{N}_{L}(i),-\operatorname{sign}\left(V_{i}^{\prime}\right), \operatorname{sign}\left(V_{\widehat{N}_{L}(i)}^{\prime}\right)}(\partial(v) \phi)(x) .
\end{aligned}
$$

On en déduit par récurrence sur le degré de $w_{2}$ que

$$
\partial(w) \widetilde{\mathcal{A}}_{L(i), N \circ L(i)}^{i, \widehat{N}_{L}(i),-\operatorname{sign}\left(V_{i}^{\prime}\right), \operatorname{sign}\left(V_{\widehat{N}_{L}(i)}^{\prime}\right)}(\phi)(x)
$$

existe pour $x \in \mathcal{O}^{P, Q, R}$; de plus, on a l'égalité

$$
\begin{aligned}
\partial(w) \widetilde{\mathcal{A}}_{L(i), N \circ L(i)}^{i, \widehat{N}_{L}(i),-\operatorname{sign}\left(V_{i}^{\prime}\right), \operatorname{sign}\left(V_{\widehat{N}_{L}(i)}^{\prime}\right)}(\phi)(x) & \\
= & \partial\left(w_{1}\right) \widetilde{\mathcal{A}}_{L(i), N \circ L(i)}^{i, \widehat{N}_{L}(i),-\operatorname{sign}\left(V_{i}^{\prime}\right), \operatorname{sign}\left(V_{\widehat{N}_{L}(i)}^{\prime}\right)}\left(\partial\left(w_{2}\right) \phi\right)(x) .
\end{aligned}
$$

On obtient ainsi

$$
\widetilde{\mathcal{A}}_{L(i), N \circ L(i)}^{i, \widehat{N}_{L}(i),-\operatorname{sign}\left(V_{i}^{\prime}\right), \operatorname{sign}\left(V_{\widehat{N}_{L}(i)}^{\prime}\right)}(\phi) \in \mathfrak{F}_{\varnothing, B}\left(P \backslash\{i\}, Q, R, d^{\prime}\right)
$$

Soit $\mathcal{K}$ une partie compacte décomposable de $\mathfrak{h}_{P \backslash\{i\}, Q, R}$ de $i^{\text {ème }}$ composante $\mathcal{K}^{\prime}$ et $\mathcal{K}^{\prime \prime}$ la partie décomposable de $\mathfrak{h}_{P, Q, R}$ telle que $\mathcal{K}_{j}^{\prime \prime}=\mathcal{K}_{j}$ pour $j \neq i$ et $\mathcal{K}_{i}^{\prime \prime}=A_{i}$; on a alors l'inégalité

$$
\sup _{x \in \mathcal{K}}\left|\partial(w) \widetilde{\mathcal{A}}_{L(i), N \circ L(i)} \phi(x)\right| \leqslant \sup _{\substack{x \in \mathcal{K}^{\prime \prime} \\ 1 \leqslant j \leqslant m}}\left|\partial\left(w_{2} v_{j}\right) \phi(x)\right| .
$$

Ainsi, les applications

$$
\begin{aligned}
\mathfrak{F}_{i, A}(P, Q, R, d) & \longrightarrow \mathfrak{F}_{i, B}\left(P \backslash\{i\}, Q, R, d^{\prime}\right) \\
\phi & \longmapsto \widetilde{\mathcal{A}}_{L(i), N \circ L(i)}^{i, \hat{N}_{L}(i),-\operatorname{sign}\left(V_{i}^{\prime}\right), \operatorname{sign}\left(V_{\widehat{N}_{L}(i)}^{\prime}\right)}(\phi)
\end{aligned}
$$

et

$$
\begin{aligned}
\mathfrak{F}_{i}(P, Q, R, d) & \longrightarrow \mathfrak{F}_{i}\left(P \backslash\{i\}, Q, R, d^{\prime}\right) \\
\phi & \widetilde{\mathcal{A}}_{L(i), N \circ L(i)}^{i, \widehat{N}_{L}(i),-\operatorname{sign}\left(V_{i}^{\prime}\right), \operatorname{sign}\left(V_{\widehat{N}_{L}(i)}^{\prime}\right)}(\phi)
\end{aligned}
$$

sont continues. Soit $\phi \in \mathfrak{F}(P, Q, R, d)$. On rappelle que l'on a l'inclusion d'espaces topologiques

$$
\mathfrak{F}(P, Q, R, d) \subset \mathfrak{F}_{i}(P, Q, R, d) .
$$

De plus, on observe que

$$
\tilde{\mathcal{A}}_{L(i), N \circ L(i)}^{i, \widehat{N}_{L}(i),-\operatorname{sign}\left(V_{i}^{\prime}\right), \operatorname{sign}\left(V_{\widehat{N}_{L}(i)}^{\prime}\right)}(\phi)
$$

appartient à $\mathfrak{F}\left(P \backslash\{i\}, Q, R, d^{\prime}\right)$. Comme la topologie de $\mathfrak{F}\left(P \backslash\{i\}, Q, R, d^{\prime}\right)$ est la topologie induite de $\mathfrak{F}_{\varnothing}\left(P \backslash\{i\}, Q, R, d^{\prime}\right)$, on en déduit que l'application

$$
\begin{aligned}
\mathfrak{F}(P, Q, R, d) & \longrightarrow \mathfrak{F}\left(P \backslash\{i\}, Q, R, d^{\prime}\right) \\
\phi & \longmapsto \widetilde{\mathcal{A}}_{L(i), N \circ L(i)}^{i, \widehat{N}_{L}(i),-\operatorname{sign}\left(V_{i}^{\prime}\right), \operatorname{sign}\left(V_{\widehat{N}_{L}(i)}^{\prime}\right)}(\phi)
\end{aligned}
$$


est continue. Le lemme est démontré.

On souhaite étendre $\widetilde{\mathcal{B}}_{L(i), M \circ L(i)}^{i, \widehat{M}_{L}(i)}$ à $\mathfrak{F}(P, Q, R, d)$ pour $i \in Q$. Soit $x \in \mathcal{O}^{P, Q, R}$. On écrit alors $x=x_{1}+x_{2}$ où $x_{1} \in \mathfrak{h}_{M, L(i), M \circ L(i)}$ et $x_{2}$ appartient au complémentaire de $\mathfrak{h}_{M, L(i), M \circ L(i)}$ suivant la décomposition (5.1.7). On note $\phi\left(.+x_{2}\right)$ l'application définie $\operatorname{sur} \mathfrak{h}_{M, L(i), M \circ L(i)}$ par

$$
\phi\left(.+x_{2}\right)(a)=\phi\left(a+x_{2}\right)
$$

On observe que l'application $\phi\left(.+x_{2}\right)$ appartient à $\mathcal{W}_{c}\left(\mathfrak{h}_{M, L(i), M \circ L(i)}, d, \varnothing\right)$, ainsi

$$
x \longmapsto \widetilde{\mathcal{B}}_{L(i), M \circ L(i)}^{i, \widehat{M}_{L}(i)}\left(\phi\left(.+x_{2}\right)\right)\left(x_{1}\right)
$$

est une fonction définie sur $\mathcal{O}^{P, Q \backslash\{i\}, R}$. On note cette fonction

$$
\widetilde{\mathcal{B}}_{L(i), M \circ L(i)}^{i, \widehat{M}_{L}(i)}(\phi)
$$

Lemme 5.1.22. - Pour $i \in Q$ et $\phi \in \mathfrak{F}(P, Q, R, d)$, on a

$$
\widetilde{\mathcal{B}}_{L(i), M \circ L(i)}^{i, \widehat{M}_{L}(i)}(\phi) \in \mathfrak{F}\left(P, Q \backslash\{i\}, R, d^{\prime}\right)
$$

et l'application linéaire

$$
\widetilde{\mathcal{B}}_{L(i), M \circ L(i)}^{i, \widehat{M}_{L}(i)}(\phi): \mathfrak{F}(P, Q, R, d) \longrightarrow \mathfrak{F}\left(P, Q \backslash\{i\}, R, d^{\prime}\right)
$$

est continue.

Démonstration. - Soit $A$ une partie adaptée de $\mathfrak{h}_{P, Q, R}$ et $B$ la partie adaptée de $\mathfrak{h}_{P, Q \backslash\{i\}, R}$ telle que $B_{j}=A_{j}$ pour $j \neq i$. On considère $\phi \in \mathfrak{F}_{i}(P, Q, R, d)$. Montrons que la fonction $\widetilde{\mathcal{B}}_{L(i), M \circ L(i)}^{i, \widehat{M}_{L}(i)}(\phi)$ appartient à $\mathfrak{F}_{\varnothing, B}(P, Q \backslash\{i\}, R, d)$. On observe tout d'abord que

$$
\operatorname{Supp}\left(\widetilde{\mathcal{B}}_{L(i), M \circ L(i)}^{i, \widehat{M}_{L}(i)}(\phi)\right) \subset B
$$

Soit $w=w_{1} w_{2} \in \operatorname{Sym}\left(\mathfrak{h}_{P, Q \backslash\{i\}, R}\right)$ de degré inférieur à $d^{\prime}$ avec $w_{1} \in \operatorname{Sym}\left(\mathfrak{h}_{\varnothing, i, \widehat{M}_{L}(i), \mathbb{C}}^{\prime}\right)$ et $w_{2}$ indépendant de $x_{i}$ et $x_{\widehat{M}_{L}(i)}$. Soit $\mathcal{K}$ une partie compacte de $\mathfrak{h}_{\varnothing, i, \widehat{M}_{L}(i)}$. D'après le lemme 5.1.10, il existe une famille $\left(v_{j}\right)_{1 \leqslant j \leqslant m}$ de $\operatorname{Sym}\left(\mathfrak{h}_{M, L(i), M \circ L(i), \mathbb{C}}\right)$ de degré au plus $\max \left(3,3 \operatorname{deg}\left(w_{1}\right)\right)$ telle que

$$
\sup _{x \in \mathcal{K}}\left|\partial\left(w_{1}\right) \widetilde{\mathcal{B}}_{L(i), M \circ L(i)}^{i, \widehat{M}_{L}(i)}(\psi)(x)\right| \leqslant \sup _{1 \leqslant j \leqslant m, x \in A_{i}}\left|\partial\left(v_{j}\right) \psi(x)\right|
$$

pour tout $\psi \in \mathcal{W}_{c}\left(\mathfrak{h}_{M, L(i), M \circ L(i)}, d, \varnothing\right)$. On observe ensuite que

$$
\phi\left(.+x_{2}\right) \in \mathcal{W}_{c}\left(\mathfrak{h}_{M, L(i), M \circ L(i)}, d, \varnothing\right) .
$$

En considérant l'inégalité (5.1.9) pour $\psi=\phi\left(.+x_{2}\right)$, on obtient que

$$
\partial\left(w_{1}\right) \widetilde{\mathcal{B}}_{L(i), M \circ L(i)}^{i, \widehat{M}_{L}(i)}\left(\phi\left(.+x_{2}\right)\right)
$$


se prolonge par continuité sur l'adhérence des composantes connexes de $\mathcal{O}^{P, Q, R}$ dans $\mathfrak{h}_{P, Q, R}$. Supposons que $\operatorname{deg}\left(w_{2}\right)>0$. Soit $v$ appartenant au complémentaire de $\mathfrak{h}_{M, L(i), M \circ L(i)}$ suivant la décomposition (5.1.7). Comme

$$
\frac{\phi\left(.+x_{2}+t v\right)-\phi\left(.+x_{2}\right)}{t}-\partial(v) \phi\left(.+x_{2}\right) \stackrel{t \rightarrow 0}{\longmapsto} 0
$$

dans $\mathcal{W}_{c}\left(\mathfrak{h}_{M, L(i), M \circ L(i)}, d, \varnothing\right)$. On en déduit que

$$
\partial\left(v w_{1}\right) \widetilde{\mathcal{B}}_{L(i), M \circ L(i)}^{i, \widehat{M}_{L}(i)}(\phi)(x)
$$

existe pour $x \in \mathcal{O}^{P, Q, R}$ de plus, on a l'égalité

$$
\partial\left(v w_{1}\right) \widetilde{\mathcal{B}}_{L(i), M \circ L(i)}^{i, \widehat{M}_{L}(i)}(\phi)(x)=\partial\left(w_{1}\right) \widetilde{\mathcal{B}}_{L(i), M \circ L(i)}^{i, \widehat{M}_{L}(i)}(\partial(v) \phi)(x) .
$$

On obtient par récurrence sur le degré de $w_{2}$ que

$$
\partial(w) \widetilde{\mathcal{B}}_{L(i), M \circ L(i)}^{i, \widehat{M}_{L}(i)}(\phi)(x)
$$

existe pour $x \in \mathcal{O}^{P, Q, R}$ de plus, on a l'égalité

$$
\partial(w) \widetilde{\mathcal{B}}_{L(i), M \circ L(i)}^{i, \widehat{M}_{L}(i)}(\phi)(x)=\partial\left(w_{1}\right) \widetilde{\mathcal{B}}_{L(i), M \circ L(i)}^{i, \widehat{M}_{L}(i)}\left(\partial\left(w_{2}\right) \phi\right)(x) .
$$

Ainsi, on a

$$
\widetilde{\mathcal{B}}_{L(i), M \circ L(i)}^{i, \widehat{M}_{L}(i)}(\phi) \in \mathfrak{F}_{\varnothing}\left(P \backslash\{i\}, Q, R, d^{\prime}\right) .
$$

Soit $\mathcal{K}$ une partie compacte décomposable de $\mathfrak{h}_{P \backslash\{i\}, Q, R}$ de $i^{\text {ème }}$ composante $\mathcal{K}^{\prime}$ et $\mathcal{K}^{\prime \prime}$ la partie décomposable de $\mathfrak{h}_{P, Q, R}$ telle que $\mathcal{K}_{j}^{\prime \prime}=\mathcal{K}_{j}^{\prime}$ pour $j \neq i$ et $\mathcal{K}_{i}^{\prime \prime}=A_{i}$; on a alors l'inégalité

$$
\sup _{x \in \mathcal{K}}\left|\partial(w) \widetilde{\mathcal{B}}_{L(i), M \circ L(i)}^{i, \widehat{M}_{L}(i)} \phi(x)\right| \leqslant \sup _{\substack{x \in \mathcal{K}^{\prime \prime} \\ 1 \leqslant j \leqslant m}}\left|\partial\left(w_{2} v_{j}\right) \phi(x)\right| .
$$

Ainsi, les applications

$$
\begin{aligned}
\mathfrak{F}_{i, A}(P, Q, R, d) & \longrightarrow \mathfrak{F}_{\varnothing, B}\left(P, Q \backslash\{i\}, R, d^{\prime}\right) \\
\phi & \longmapsto \widetilde{\mathcal{B}}_{L(i), M \circ L(i)}^{i, \widetilde{M}_{L}(i)}(\phi)
\end{aligned}
$$

et

$$
\begin{aligned}
\mathfrak{F}_{i}(P, Q, R, d) & \longrightarrow \mathfrak{F}_{\varnothing}\left(P, Q \backslash\{i\}, R, d^{\prime}\right) \\
\phi & \longmapsto \widetilde{\mathcal{B}}_{L(i), N \circ L(i)}^{i, \widehat{N}_{L}(i)}(\phi)
\end{aligned}
$$

sont continues. Soit $\phi \in \mathfrak{F}(P, Q, R, d)$. On rappelle que l'on a l'inclusion d'espaces topologiques

$$
\mathfrak{F}(P, Q, R, d) \subset \mathfrak{F}_{i}(P, Q, R, d) .
$$

De plus, on observe que

$$
\widetilde{\mathcal{B}}_{L(i), M \circ L(i)}^{i, \widehat{M}_{L}(i)}(\phi)
$$

appartient à $\mathfrak{F}\left(P, Q \backslash\{i\}, R, d^{\prime}\right)$. Comme la topologie de $\mathfrak{F}\left(P, Q \backslash\{i\}, R, d^{\prime}\right)$ est la topologie induite de $\mathfrak{F}_{\varnothing}\left(P, Q \backslash\{i\}, R, d^{\prime}\right)$, on en déduit que l'application

$$
\begin{aligned}
\mathfrak{F}(P, Q, R, d) & \longrightarrow \mathfrak{F}\left(P, Q \backslash\{i\}, R, d^{\prime}\right) \\
\phi & \longmapsto \widetilde{\mathcal{B}}_{L(i), M \circ L(i)}^{i, \widehat{M}_{L}(i)}(\phi)
\end{aligned}
$$


est continue.

On souhaite ici aussi étendre $\mathcal{C}_{L(i)}^{i,-\operatorname{sign}\left(V_{i}^{\prime}\right)}$ à $\mathfrak{F}(P, Q, R, d)$ pour $i \in R$. Soit $x \in$ $\mathcal{O}^{P, Q, R}$. On écrit alors $x=x_{1}+x_{2}$ où $x_{1} \in \mathfrak{h}_{M, L(i)}$ et $x_{2}$ appartient au complémentaire de $\mathfrak{h}_{M, L(i)}$ suivant la décomposition (5.1.7). On note $\phi\left(.+x_{2}\right)$ l'application définie sur $\mathfrak{h}_{M, L(i)}$ par

$$
\phi\left(.+x_{2}\right)(a)=\phi\left(a+x_{2}\right)
$$

On observe que l'application $\phi\left(.+x_{2}\right)$ appartient à $\mathcal{W}_{c}\left(\mathfrak{h}_{M, L(i)}\right)$, ainsi

$$
x \longmapsto \mathcal{C}_{L(i)}^{i,-\operatorname{sign}\left(V_{i}^{\prime}\right)}\left(\phi\left(.+x_{2}\right)\right)\left(x_{1}\right)
$$

est une fonction définie sur $\mathcal{O}^{P, Q}, R \backslash\{i\}$. On note cette fonction

$$
\mathcal{C}_{L(i)}^{i,-\operatorname{sign}\left(V_{i}^{\prime}\right)}(\phi)
$$

Lemme 5.1.23. - Pour $i \in R$ et $\phi \in \mathfrak{F}(P, Q, R, d)$, on a

$$
\mathcal{C}_{L(i)}^{i,-\operatorname{sign}\left(V_{i}^{\prime}\right)}(\phi) \in \mathfrak{F}\left(P, Q, R \backslash\{i\}, d^{\prime}\right)
$$

et l'application linéaire

$$
\mathcal{C}_{L(i)}^{i,-\operatorname{sign}\left(V_{i}^{\prime}\right)}(\phi): \mathfrak{F}(P, Q, R, d) \longrightarrow \mathfrak{F}\left(P, Q, R \backslash\{i\}, d^{\prime}\right)
$$

est continue.

Démonstration. - Soit $A$ une partie adaptée de $\mathfrak{h}_{P, Q, R}$ et $B$ la partie adaptée de $\mathfrak{h}_{P, Q, R \backslash\{i\}}$ telle que $B_{j}=A_{j}$ pour $j \neq i$. On considère $\phi \in \mathfrak{F}_{\varnothing}(P, Q, R, d)$ Montrons que la fonction $\mathcal{C}_{L(i)}^{i,-\operatorname{sign}\left(V_{i}^{\prime}\right)}(\phi)$ appartient à $\mathfrak{F}_{\varnothing}(P, Q, R \backslash\{i\}, d)$. On observe tout d'abord que

$$
\operatorname{Supp}\left(\mathcal{C}_{L(i)}^{i,-\operatorname{sign}\left(V_{i}^{\prime}\right)}(\phi)\right) \subset B
$$

Soit $w=w_{1} w_{2} \in \operatorname{Sym}\left(\mathfrak{h}_{P, Q, R \backslash\{i\}, \mathbb{C}}\right)$ de degré inférieur à $d^{\prime}$ avec $w_{1} \in \operatorname{Sym}\left(\mathfrak{h}_{\varnothing, i, \mathbb{C}}^{\prime}\right)$ et $w_{2}$ indépendant de $x_{i}$. Soient $\mathcal{K}_{i}$ une partie compacte de $\mathfrak{h}_{M, L(i)}$ et $\mathcal{K}$ une partie compacte de $\mathfrak{h}_{\varnothing, i}^{\prime}$. D'après le lemme 5.1.14, il existe une famille $\left(v_{j}\right)_{1 \leqslant j \leqslant m}$ de $\operatorname{Sym}\left(\mathfrak{h}_{M, L(i), \mathbb{C}}\right)$ de degré au plus $\max \left(3,3 \operatorname{deg}\left(w_{1}\right)\right)$ telle que

$$
\sup _{x \in \mathcal{K}}\left|\partial\left(w_{1}\right) \mathcal{C}_{L(i)}^{i,-\operatorname{sign}\left(V_{i}^{\prime}\right)}(\psi)(x)\right| \leqslant \sup _{1 \leqslant j \leqslant m, x \in \mathcal{K}_{j}}\left|\partial\left(v_{j}\right) \psi(x)\right|
$$

pour tout $\psi \in \mathcal{W}_{c}\left(\mathfrak{h}_{M, L(i)}, d\right)$. On observe ensuite que

$$
\phi\left(.+x_{2}\right) \in \mathcal{W}_{c}\left(\mathfrak{h}_{M, L(i)}, d\right) .
$$

En considérant l'inégalité (5.1.10) pour $\psi=\phi\left(.+x_{2}\right)$, on obtient que

$$
\partial\left(w_{1}\right) \mathcal{C}_{L(i)}^{i,-\operatorname{sign}\left(V_{i}^{\prime}\right)}\left(\phi\left(.+x_{2}\right)\right)
$$


se prolonge par continuité sur l'adhérence des composantes connexes de $\mathcal{O}^{P, Q, R \backslash\{i\}}$. Supposons que $\operatorname{deg}\left(w_{2}\right)>0$. Soit $v$ appartenant au complémentaire de $\mathfrak{h}_{M, L(i)}$ suivant la décomposition (5.1.7). Comme

$$
\frac{\phi\left(.+x_{2}+t v\right)-\phi\left(.+x_{2}\right)}{t}-\partial(v) \phi\left(.+x_{2}\right) \stackrel{t \rightarrow 0}{\longmapsto} 0
$$

dans $\mathcal{W}_{c}\left(\mathfrak{h}_{M, L(i)}, d\right)$. On en déduit que

$$
\partial\left(v w_{1}\right) \mathcal{C}_{L(i)}^{i,-\operatorname{sign}\left(V_{i}^{\prime}\right)}(\phi)(x)
$$

existe pour $x \in \mathcal{O}^{P, Q, R}$ de plus, on a l'égalité

$$
\partial\left(v w_{1}\right) \mathcal{C}_{L(i)}^{i,-\operatorname{sign}\left(V_{i}^{\prime}\right)}(\phi)(x)=\partial\left(w_{1}\right) \mathcal{C}_{L(i)}^{i,-\operatorname{sign}\left(V_{i}^{\prime}\right)}(\partial(v) \phi)(x) .
$$

On en déduit par récurrence sur le degré de $w_{2}$ que

$$
\partial(w) \mathcal{C}_{L(i)}^{i,-\operatorname{sign}\left(V_{i}^{\prime}\right)}(\phi)(x)
$$

existe pour $x \in \mathcal{O}^{P, Q, R}$ de plus, on a l'égalité

$$
\partial(w) \mathcal{C}_{L(i)}^{i,-\operatorname{sign}\left(V_{i}^{\prime}\right)}(\phi)(x)=\partial\left(w_{1}\right) \mathcal{C}_{L(i)}^{i,-\operatorname{sign}\left(V_{i}^{\prime}\right)}\left(\partial\left(w_{2}\right) \phi\right)(x) .
$$

Ainsi, on a

$$
\mathcal{C}_{L(i)}^{i,-\operatorname{sign}\left(V_{i}^{\prime}\right)}(\phi) \in \mathfrak{F}_{\varnothing}\left(P, Q, R \backslash\{i\}, d^{\prime}\right) .
$$

Soit $\mathcal{K}$ une partie compacte décomposable de $\mathfrak{h}_{P, Q, R \backslash\{i\}}$ de $i^{\text {ème }}$ composante $\mathcal{K}^{\prime}$ et $\mathcal{K}^{\prime \prime}$ la partie décomposable de $\mathfrak{h}_{P, Q, R}$ telle que $\mathcal{K}_{j}^{\prime \prime}=\mathcal{K}_{j}$ pour $j \neq i$ et $\mathcal{K}_{i}^{\prime \prime}=A_{i}$; on a alors l'inégalité

$$
\sup _{x \in \mathcal{K}}\left|\partial(w) \mathcal{C}_{L(i)}^{i,-\operatorname{sign}\left(V_{i}^{\prime}\right)} \phi(x)\right| \leqslant \sup _{\substack{x \in \mathcal{K}^{\prime \prime} \\ 1 \leqslant j \leqslant m}}\left|\partial\left(w_{2} v_{j}\right) \phi(x)\right| .
$$

Ainsi, les applications

$$
\begin{aligned}
\mathfrak{F}_{\varnothing, A}(P, Q, R, d) & \longrightarrow \mathfrak{F}_{\varnothing, B}\left(P, Q, R \backslash\{i\}, d^{\prime}\right) \\
\phi & \longmapsto \mathcal{C}_{L(i)}^{i,-\operatorname{sign}\left(V_{i}^{\prime}\right)}(\phi)
\end{aligned}
$$

et

$$
\begin{aligned}
\mathfrak{F}_{\varnothing}(P, Q, R, d) & \longrightarrow \mathfrak{F}_{\varnothing}\left(P, Q, R \backslash\{i\}, d^{\prime}\right) \\
\phi & \longmapsto \mathcal{C}_{L(i)}^{i,-\operatorname{sign}\left(V_{i}^{\prime}\right)}(\phi)
\end{aligned}
$$

sont continues. Soit $\phi \in \mathfrak{F}(P, Q, R, d)$. On rappelle que l'on a l'inclusion d'espaces topologiques

$$
\mathfrak{F}(P, Q, R, d) \subset \mathfrak{F}_{\varnothing}(P, Q, R, d) .
$$

De plus, on observe que

$$
\mathcal{C}_{L(i)}^{i,-\operatorname{sign}\left(V_{i}^{\prime}\right)}(\phi)
$$

appartient à $\mathfrak{F}\left(P, Q, R \backslash\{i\}, d^{\prime}\right)$. Comme la topologie de $\mathfrak{F}\left(P, Q, R \backslash\{i\}, d^{\prime}\right)$ est la topologie induite de $\mathfrak{F}_{\varnothing}\left(P, Q, R \backslash\{i\}, d^{\prime}\right)$, on en déduit que l'application

$$
\begin{aligned}
\mathfrak{F}(P, Q, R, d) & \longrightarrow \mathfrak{F}\left(P, Q, R \backslash\{i\}, d^{\prime}\right) \\
\phi & \longmapsto \mathcal{C}_{L(i)}^{i,-\operatorname{sign}\left(V_{i}^{\prime}\right)}(\phi)
\end{aligned}
$$


est continue.

Démonstration de la proposition 5.1.18. - On rappelle que l'on a l'égalité d'espaces topologiques

$$
\mathcal{W}_{c}\left(\mathfrak{h}_{M}, d, \Gamma_{N}\right)=\mathfrak{F}\left(I_{N, L}, I_{M, L}, K_{M, N, L}, d\right) .
$$

Soit $A$ une partie adaptée de $\mathfrak{h}_{M}$, donc en particulier compacte. On considère une fonction $\phi \in \mathcal{W}_{A}\left(\mathfrak{h}_{M}, d, \Gamma_{N}\right)$. Pour $L \in \mathfrak{S}_{\mathrm{n}}$, on a l'égalité suivante

$$
\begin{aligned}
\int_{\check{\mathfrak{h}}_{M}} & \frac{\phi(x)}{\prod_{i \in\{1, \ldots, \mathrm{n}\}}\left(c_{M}^{-1}(x)_{L(i)}+\mathrm{ity}_{M L(i)}^{L}-x_{i}^{\prime}\right)} d x \\
= & \prod_{i \in I_{M, L}} \mathcal{B}_{L(i), M \circ L(i)}^{i, \widehat{M}_{L}(i)} \prod_{i \in I_{N, L}} \mathcal{A}_{L(i), N \circ L(i)}^{i, \widehat{N}_{L}(i),-\operatorname{sign}\left(V_{i}^{\prime}\right), \operatorname{sign}\left(V_{\widehat{N}_{L}(i)}^{\prime}\right), t} \prod R_{M, N, L}^{t}(\phi)
\end{aligned}
$$

où

$$
R_{M, N, L}^{t}(\phi)=\prod_{i \in K_{M, N, L}} \mathcal{C}_{L(i)}^{i,-\operatorname{sign}\left(V_{i}^{\prime}\right), t}(\phi) .
$$

et $\prod$ représente la composée des applications. On suppose maintenant que $L \in$ $\operatorname{Pr}(M, N)$ et on somme l'expression (5.1.11) sur les éléments $L^{\prime}$ de la classe de $L$ pondéré de la signature $\varepsilon\left(L^{\prime}\right)$, on obtient :

$$
\begin{aligned}
& \mathcal{T}_{M, N, \bar{L}_{M, N}}^{t}(\phi) \\
& =\sum_{L^{\prime} \in \bar{L}_{M, N}} \varepsilon\left(L^{\prime}\right) \int_{\check{\mathfrak{h}}_{M}} \frac{\phi(x)}{\prod_{i \in\{1, \ldots, \mathrm{n}\}}\left(c_{M}^{-1}(x)_{L^{\prime}(i)}+\mathrm{i} t y_{M L^{\prime}(i)}^{L^{\prime}}-x_{i}^{\prime}\right)} d x \\
& =\varepsilon(L) \prod_{i \in I_{M, L}} \widetilde{\mathcal{B}}_{L(i), M \circ L(i)}^{i, \widehat{M}_{L}(i)} \prod_{i \in I_{N, L}} \widetilde{\mathcal{A}}_{L(i), N \circ L(i)}^{i, \widehat{N}_{L}(i),-\operatorname{sign}\left(V_{i}^{\prime}\right), \operatorname{sign}\left(V_{\widehat{N}_{L}(i)}^{\prime}\right), t} \prod R_{M, N, L}^{t}(\phi)
\end{aligned}
$$

où la définition de $R_{M, N, L}^{t}(\phi)$ est inchangée et ne dépend que de $\bar{L}_{M, N}$. Montrons que l'on peut prendre la limite en $t=0$ de l'expression (5.1.12). Soit $\left(t_{n}\right)_{n \in \mathbb{N}}$ une suite de réels strictement positifs de limite nulle et $\mathcal{K}$ une partie compacte décomposable de $\mathfrak{h}_{M}$. Soit $x \in \mathfrak{h}_{\varnothing}^{\prime}\left(=\mathfrak{h}_{\varnothing, \varnothing, \varnothing)}\right.$, on remarque que $\mathfrak{h}_{\varnothing}^{\prime}$ est décomposable; pour $i \in$ $I_{N, L} \cup I_{M, L} \cup K_{M, N, L}$, on note $x_{i} \in \mathfrak{h}_{\varnothing, i}^{\prime}$ tels que $x=\sum x_{i}$. D'après le théorème de Banach-Steinhaus, les familles de formes linéaires

$$
\left(\widetilde{\mathcal{A}}_{L(i), N \circ L(i)}^{i, \tilde{N}(i),-\operatorname{sign}\left(V_{i}^{\prime}\right), \operatorname{sign}\left(V_{\widetilde{N}(i)}\right), t_{n}}(\cdot)\left(x_{i}\right)\right)_{n \in \mathbb{N}} \quad \text { et } \quad\left(\mathcal{C}_{L(i)}^{i,-\operatorname{sign}\left(V_{i}^{\prime}\right), t_{n}}(\cdot)\left(x_{i}\right)\right)_{n \in \mathbb{N}}
$$

sont équicontinues respectivement sur les espaces $\mathcal{W}_{\mathcal{K}_{i}}\left(\mathfrak{h}_{M, L(i), N \circ L(i)}, d, \Gamma_{N}\right)$ et $\mathcal{W}_{\mathcal{K}_{i}}\left(\mathfrak{h}_{M, L(i)}, d\right)$. Cela permet de montrer que la famille de formes linéaires

$$
\left(\prod_{i \in I_{M, L}} \widetilde{\mathcal{B}}_{L(i), M \circ L(i)}^{i, \widehat{M}_{L}(i)} \prod_{i \in I_{N, L}} \widetilde{\mathcal{A}}_{L(i), N \circ L(i)}^{i, \widehat{N}_{L}(i),-\operatorname{sign}\left(V_{i}^{\prime}\right), \operatorname{sign}\left(V_{\widehat{N}_{L}(i)}^{\prime}\right), t} \prod R_{M, N, L}^{t}(\cdot)(x)\right)_{n \in \mathbb{N}}
$$


est équicontinue $\operatorname{sur} \mathcal{W}_{\mathcal{K}}\left(\mathfrak{h}_{M}, d, \Gamma_{N}\right)$. On en déduit que

$$
\begin{aligned}
& \lim _{t \rightarrow 0} \mathcal{T}_{M, N, \bar{L}_{M, N}}^{t}(\phi) \\
= & \varepsilon(L) \prod_{i \in I_{M, L}} \widetilde{\mathcal{B}}_{L(i), M \circ L(i)}^{i, \widehat{M}_{L}(i)} \prod_{i \in I_{N, L}} \widetilde{\mathcal{A}}_{L(i), N \circ L(i)}^{i, \widehat{N}_{L}(i),-\operatorname{sign}\left(V_{i}^{\prime}\right), \operatorname{sign}\left(V_{\widehat{N}_{L}(i)}^{\prime}\right)} \prod R_{M, N, L}(\phi) .
\end{aligned}
$$

On note cette expression $\mathcal{T}_{M, N, \bar{L}_{M, N}}(\phi)$. D'après les propositions 5.1.7, 5.1.10 et 5.1.14, on obtient que $\mathcal{T}_{M, N, \bar{L}_{M, N}}(\phi) \in \mathcal{Z}\left(\mathfrak{h}_{\varnothing}^{\prime}, d^{\prime}\right)$ mais aussi que l'application

$$
\begin{aligned}
\mathcal{T}_{M, N, \bar{L}_{M, N}}: \mathcal{W}_{c}\left(\mathfrak{h}_{M}, d, \Gamma_{N}\right) & \longrightarrow \mathcal{Z}\left(\mathfrak{h}_{\varnothing}^{\prime}, d^{\prime}\right) \\
\phi & \longmapsto \mathcal{T}_{M, N, \bar{L}_{M, N}}(\phi)
\end{aligned}
$$

est définie et continue. Il reste ensuite à observer que

$$
\mathcal{R}_{M}^{t}(\phi)=\sum \mathcal{T}_{M, N, \bar{L}_{M, N}}^{t}(\phi)
$$

où la somme s'effectue sur les classes d'équivalences de $\simeq_{M, N}$. Comme $\mathcal{T}_{M, N, \bar{L}_{M, N}}$ est continue, $\mathcal{R}_{M}$ est aussi une application continue.

Corollaire 5.1.24. - Avec les notations du lemme précédent, on a pour $\phi \in$ $\mathcal{W}_{c}\left(\mathfrak{h}_{M}, d, \Gamma_{N}\right)$

$$
\begin{aligned}
& \mathcal{T}_{M, N, \bar{L}_{M, N}}(\phi) \\
= & \varepsilon(L) \prod_{i \in I_{M, L}} \widetilde{\mathcal{B}}_{L(i), M \circ L(i)}^{i, \widehat{M}_{L}(i)} \prod_{i \in I_{N, L}} \widetilde{\mathcal{A}}_{L(i), N \circ L(i)}^{i, \widehat{N}_{L}(i),-\operatorname{sign}\left(V_{i}^{\prime}\right), \operatorname{sign}\left(V_{\widehat{N}_{L}(i)}^{\prime}\right)} \prod R_{M, N, L}(\phi) .
\end{aligned}
$$

et l'application

est continue.

$$
\begin{aligned}
\mathcal{W}_{c}\left(\mathfrak{h}_{M}, d, \Gamma_{N}\right) & \longmapsto \mathcal{Z}\left(\mathfrak{h}_{\varnothing}^{\prime}, d^{\prime}\right) \\
\phi & \longmapsto \mathcal{T}_{M, N, \bar{L}_{M, N}}(\phi)
\end{aligned}
$$

REMARQUE. - Les $\prod$ représentent la composée des applications.

Démonstration. - Cette égalité découle de l'égalité (5.1.13).

Le résultat suivant montre les points (1) et (2) du théorème 5.1.1.

Proposition 5.1.25. - Pour $\phi \in \mathcal{D}(\mathfrak{g})$, on a les propriétés suivantes :

(1) La fonction

$$
x^{\prime} \in \mathfrak{h}_{\varnothing}^{\prime \text { reg }} \longmapsto \mathbf{C h c}(\phi)_{\varnothing}\left(x^{\prime}\right)
$$

appartient ̀̀ $\mathcal{Z}\left(\mathfrak{h}_{\varnothing}^{\prime}\right)$.

(2) L'application

$$
\begin{aligned}
\mathcal{D}(\mathfrak{g}) & \longrightarrow \mathcal{Z}\left(\mathfrak{h}_{\varnothing}^{\prime}\right) \\
\phi & \longmapsto \operatorname{Chc}(\phi)_{\varnothing}
\end{aligned}
$$

est continue. 
Démonstration. - Pour $M \in \Omega$, on rappelle que $\phi_{M}$ est l'intégrale invariante de Harish-Chandra de $\phi \in \mathcal{D}(\mathfrak{g})$. On considère $d, d^{\prime} \in \mathbb{N}$ tels que $9 \leqslant 3 d^{\prime} \leqslant d$. D'après la proposition $4.3 .12, \phi_{M}$ appartient à $\widetilde{\mathcal{W}}_{c}\left(\mathfrak{h}_{M}, d, I_{M} \times J_{M}\right)$. Pour chaque $N \in \Omega\left(I_{M} \cup J_{M}\right)$, il existe une application continue

$$
v_{M, N}: \widetilde{\mathcal{W}}_{c}\left(\mathfrak{h}_{M}, d, I_{M} \times J_{M}\right) \longrightarrow \widetilde{\mathcal{W}}_{c}\left(\mathfrak{h}_{M}, d, \Gamma_{N}\right)
$$

telles que

$$
\sum_{N \in \Omega\left(I_{M} \cup J_{M}\right)} v_{M, N}=i d_{\widetilde{\mathcal{W}}_{c}\left(\mathfrak{h}_{M}, d, I_{M} \times J_{M}\right)}
$$

d'après la proposition 4.3.14. D'après la proposition 4.1.7, on a en prenant $p=0$

$$
\operatorname{Chc}(\phi)_{\varnothing}\left(x^{\prime}\right)=\frac{C_{\mathfrak{g}, \mathfrak{g}^{\prime}}}{\mathrm{p} ! \mathbf{q} !} \sum_{(M, L) \in \Omega \times \mathfrak{S}_{n}} \lim _{t \rightarrow 0, t>0} \int_{\check{\mathfrak{h}}_{M}} \nu_{L, x^{\prime}, M}^{t}(\phi),
$$

ainsi on a la relation pour $x^{\prime} \in \mathfrak{h}_{\varnothing}^{\text {reg }}$ et $\phi \in \mathcal{D}(\mathfrak{g})$

$$
\operatorname{Chc}(\phi)_{\varnothing}\left(x^{\prime}\right)=C_{\mathfrak{g}, \mathfrak{g}^{\prime}}^{\prime} \sum_{M \in \Omega} \mathcal{R}_{M}\left(\phi_{M}\right) .
$$

où $C_{\mathfrak{g}, \mathfrak{g}^{\prime}}^{\prime}=(-1)^{\mathrm{n}(\mathrm{n}-1) / 2} \frac{C_{\mathfrak{g}, \mathfrak{g}^{\prime}}}{\mathrm{p} ! \mathrm{q} !}$. D'après le corollaire 2.2 .2 , on a

$$
\nu_{L, x^{\prime}, M}^{t}(\phi)=(-1)^{\mathrm{n}(\mathrm{n}-1) / 2} \varepsilon(L) \frac{\phi_{M}}{\prod_{i \in\{1, \ldots, \mathrm{n}\}}\left(c_{M}^{-1}(x)_{L(i)}+\mathrm{i} t y_{M L(i)}^{L}-x_{i}^{\prime}\right)} \mu_{M} .
$$

On obtient ainsi l'égalité

$$
\sum_{\substack{M \in \Omega \\ L \in \mathfrak{S}_{n}}} \int_{\check{\mathfrak{h}}_{M}} \nu_{L, x^{\prime}, M}^{t}(\phi)=(-1)^{\mathrm{n}(\mathrm{n}-1) / 2} \sum_{\substack{M \in \Omega \\ N \in \Omega\left(I_{M} \cup J_{M}\right)}} \mathcal{R}_{M}^{t}\left(v_{M, N}\left(\phi_{M}\right)\right) ;
$$

ainsi d'après le lemme 5.1 .18 , on sait que

$$
\lim _{t \rightarrow 0} \sum_{M, L} \int_{\check{\mathfrak{h}}_{M}} \nu_{L, x^{\prime}, M}^{t}(\phi)
$$

existe et définit une fonction qui appartient à $\mathcal{Z}\left(\mathfrak{h}_{\varnothing}^{\prime}, d^{\prime}\right)$. On obtient le point (1) puisque que l'on peut prendre $d^{\prime}$ aussi grand que l'on veut. Comme les applications $v_{M, N}$ et $\mathcal{R}_{M}$ sont continues d'après les propositions 4.3 .14 et 5.1 .18 , on en déduit le point (2).

Relations de sauts. - On souhaite à présent calculer les relations de sauts et ainsi expliciter le point (3) du théorème 5.1.1. Pour cela, on aura besoin de considérer certains des objets introduits jusqu'ici dans un cadre plus général. On notera par exemple $\mathfrak{h}_{V_{1}, \varnothing}$ pour désigner la sous-algèbre de Cartan diagonale compacte du sousgroupe $G\left(V_{1}\right)$ si $V_{1}$ désigne un sous-espace de $V$. On mettra en indice ou en exposant les sous-espaces de $V$ et $V^{\prime}$ auxquels sont associés les objets pour lever l'ambiguïté. On note pour des ensembles finis $A$ et $B, \Omega(A, B)$ l'ensemble des injections d'une partie de $A$ dans $B$. Pour $(a, b) \in\{1, \ldots, \mathrm{n}\}^{2}$, on note $V_{a, b}=\left\{x \in V \mid x_{a}=x_{b}=0\right\}$, 
de même pour $V^{\prime}$. La restriction du produit hermitien de $V$ à $V_{a, b}$ est non dégénérée ce qui permet de considérer le groupe $G\left(V_{a, b}\right)$. Dans un premier temps, on calcule le saut de $\mathcal{R}_{M}$.

Proposition 5.1.26. - Pour $\phi \in \widetilde{\mathcal{W}}_{c}\left(\mathfrak{h}_{M}, d, I_{M} \times J_{M}\right), i_{0}, j_{0} \in\{1, \ldots, \mathrm{n}\}$ avec $i_{0}<j_{0}$ et $w \in \mathcal{E}\left(\mathfrak{h}_{\varnothing, i_{0}, j_{0}}^{\prime}, d^{\prime}\right)$, on $a$

$$
\begin{gathered}
\left\langle\partial(w) \mathcal{R}_{M}(\phi)\left(x^{\prime}\right)\right\rangle_{i_{0}, j_{0}} \\
=\sum_{a \in A_{M}}(-1)^{i_{0}+j_{0}+a+M(a)} \mathcal{R}_{M_{1}}^{V_{a, M(a)}, V_{i_{0}, j_{0}}^{\prime}}\left(\left\langle\partial(w) \widetilde{\mathcal{B}}_{a, M(a)}^{i_{0}, j_{0}}(\phi)\right\rangle_{i_{0}, j_{0}}\right) \\
+\sum_{(a, b) \in I_{M} \times J_{M}}(-1)^{i_{0}+j_{0}+a+b} \mathcal{R}_{M}^{V_{a, b}, V_{i_{0}, j_{0}}^{\prime}}\left(\left\langle\partial(w) \widetilde{\mathcal{A}}_{a, b}^{i_{0}, j_{0},-\operatorname{sign}\left(V_{i_{0}}^{\prime}\right), \operatorname{sign}\left(V_{j_{0}}^{\prime}\right)}(\phi)\right\rangle_{i_{0}, j_{0}}\right)
\end{gathered}
$$

où $M_{1}$ désigne la restriction de $M$ à $\mathrm{A}_{M} \backslash\{a\}$. Pour démontrer cette proposition, on a besoin de deux lemmes. On fixe un couple $\left(i_{0}, j_{0}\right) \in\{1, \ldots, \mathrm{n}\}^{2}$ avec $i_{0}<j_{0}, N \in \Omega$ tel que $M$ et $N$ soient disjoints, $L \in \operatorname{Pr}(M, N)$ et $\phi \in \widetilde{\mathcal{W}}_{c}\left(\mathfrak{h}_{M}, d, \Gamma_{N}\right)$. On considère les deux parties de $\{1, \ldots, n\}^{2}$ suivantes

$$
\begin{aligned}
\Gamma_{M, L} & =\left\{(i, j) \in\{1, \ldots, \mathrm{n}\}^{2} \mid i \in I_{M, L} \text { et } \widehat{M}(i)=j\right\} \\
\Gamma_{N, L} & =\left\{(i, j) \in\{1, \ldots, \mathrm{n}\}^{2} \mid i \in I_{N, L} \text { et } \widehat{N}(i)=j\right\} .
\end{aligned}
$$

Comme $L \in \operatorname{Pr}(M, N)$, on a $i<j$ si $(i, j) \in \Gamma_{M, L} \cup \Gamma_{N, L}$. On considère le sous-espace $\mathcal{W}_{M, N}$ de $\mathcal{W}_{c}\left(\mathfrak{h}_{M}, d, \Gamma_{N}\right)$ égale à

$$
\underset{j \in \mathrm{A}_{M}}{\otimes} \mathcal{W}_{c}\left(\mathfrak{h}_{M, j, M(j)}, d\right) \underset{j \in \mathbb{A}_{N}}{\otimes} \mathcal{W}_{c}\left(\mathfrak{h}_{M, j, N(j)}, d,[j, N(j)]\right) \underset{j \in I_{M+N} \cup J_{M+N}}{\otimes} \mathcal{W}_{c}\left(\mathfrak{h}_{M, j}, d\right) .
$$

On sait d'après le Théorème 4.4.4 que $\mathcal{W}_{M, N}$ est dense dans $\mathcal{W}_{c}\left(\mathfrak{h}_{M}, d, \Gamma_{N}\right)$.

Lemme 5.1.27. - Pour $\left(i_{0}, j_{0}\right) \notin \Gamma_{M, L} \cup \Gamma_{N, L}$ et $\phi \in \mathcal{W}_{c}\left(\mathfrak{h}_{M}, d, \Gamma_{N}\right)$, on a

$$
\left\langle\partial(w) \mathcal{T}_{M, N, \bar{L}_{M, N}}(\phi)\right\rangle_{i_{0}, j_{0}}=0 .
$$

Démonstration. - Pour $x^{\prime} \in \mathfrak{h}^{\prime\left(i_{0}, j_{0}\right)}$, en utilisant le lemme 5.1.21, on observe que l'application

$$
\begin{aligned}
\mathcal{W}_{c}\left(\mathfrak{h}_{M}, d, \Gamma_{N}\right) & \longrightarrow \mathbb{C} \\
\phi & \longmapsto\left\langle\partial(w) \widetilde{\mathcal{A}}_{a, b}^{i_{0}, j_{0},-\operatorname{sign}\left(V_{i_{0}}^{\prime}\right), s i g n\left(V_{j_{0}}^{\prime}\right)}(\phi)\right\rangle_{i_{0}, j_{0}}\left(x^{\prime}\right)
\end{aligned}
$$

est une forme linéaire continue. Pour $\phi \in \mathcal{W}_{M, N}$, on a

$$
\phi=\underset{j \in \mathrm{A}_{M} \cup \mathrm{A}_{N} \cup I_{M+N} \cup J_{M+N}}{\times} \phi_{j}
$$


suivant la décomposition 5.1.16. D'après le corollaire 5.1.24, on a

$$
\begin{aligned}
& \mathcal{T}_{M, N, \bar{L}_{M, N}}(\phi) \\
& =\varepsilon(L) \prod_{i \in I_{M, L}} \widetilde{\mathcal{B}}_{L(i), M \circ L(i)}^{i, \widehat{M}(i)} \prod_{i \in I_{N, L}} \widetilde{\mathcal{A}}_{L(i), N \circ L(i)}^{i, \widehat{N}(i),-\operatorname{sign}\left(V_{i}^{\prime}\right), \operatorname{sign}\left(V_{\widehat{N}(i)}^{\prime}\right)} \prod_{i \in I_{M, N, L} \cup J_{M, N, L}} \mathcal{C}_{L(i)}^{i,-\operatorname{sign}\left(V_{i}^{\prime}\right)}(\phi) \\
& =\varepsilon(L) \underset{i \in I_{M, L}}{\times} \widetilde{\mathcal{B}}_{L(i), M \circ L(i)}^{i, \widehat{M}(i)}\left(\phi_{i}\right) \underset{i \in I_{N, L}}{\times} \widetilde{\mathcal{A}}_{L(i), N \circ L(i)}^{i, \widehat{N}(i),-\operatorname{sign}\left(V_{i}^{\prime}\right), \operatorname{sign}\left(V_{\widehat{N}(i)}^{\prime}\right)}\left(\phi_{i}\right) \\
& \underset{i \in I_{M, N, L} \cup J_{M, N, L}}{\times} \mathcal{C}_{L(i)}^{i,-\operatorname{sign}\left(V_{i}^{\prime}\right)}\left(\phi_{i}\right) .
\end{aligned}
$$

D'après les propositions 5.1.6, 5.1.9 et 5.1.13, cette fonction ne possède des sauts que sur les hyperplans

$$
\left\{x_{i}=x_{\widehat{M}(i)}\right\}_{i \in I_{M, L}} \text { et }\left\{x_{i}=x_{\widehat{N}(i)}\right\}_{i \in J_{M, L}} .
$$

Comme $\left(i_{0}, j_{0}\right) \notin \Gamma_{M, L} \cup \Gamma_{N, L}$, on en déduit le résultat pour $\phi \in \mathcal{W}_{M, N}$. On obtient le résultat pour $\phi$ quelconque en utilisant la densité de $\mathcal{W}_{M, N}$ dans $\mathcal{W}_{c}\left(\mathfrak{h}_{M}, d, \Gamma_{N}\right)$ (Théorème 4.4.4) et la continuité de $\mathcal{T}_{M, N, \bar{L}_{M, N}}$ (corollaire 5.1.24).

Lemme 5.1.28. - Pour $(a, b) \in I_{M} \times J_{M}$ tel que $b \neq N(a)$ et $\phi \in \mathcal{W}_{c}\left(\mathfrak{h}_{M}, d, \Gamma_{N}\right)$, on $a$

$$
\left\langle\partial(w) \widetilde{\mathcal{A}}_{a, b}^{i_{0}, j_{0},-\operatorname{sign}\left(V_{i_{0}}^{\prime}\right), \operatorname{sign}\left(V_{j_{0}}\right)}(\phi)\right\rangle_{i_{0}, j_{0}}=0
$$

Démonstration. - Comme les dérivées de $\phi$ ne possèdent pas de saut suivant l'hyperplan $\left\{x_{a}=x_{b}\right\}$, on a le résultat d'après le point (3) de la proposition 5.1.6.

Démonstration de la proposition 5.1.26

Si $\left(i_{0}, j_{0}\right) \notin \Gamma_{M, L} \cup \Gamma_{N, L}$, on a d'après le lemme 5.1.27

$$
\left\langle\partial(w) \mathcal{T}_{M, N, \bar{L}_{M, N}}(\phi)\right\rangle_{i_{0}, j_{0}}=0 .
$$

Si $\left(i_{0}, j_{0}\right) \in \Gamma_{M, L}$, en utilisant l'expression (5.1.14), on obtient

$$
\begin{aligned}
\left\langle\partial(w) \mathcal{T}_{M, N, L_{M, N}}(\phi)\right\rangle_{i_{0}, j_{0}}=\varepsilon(L) & \prod_{\substack{i \in I_{M, L} \\
i \neq i_{0}}} \widetilde{\mathcal{B}}_{L(i), M \circ L(i)}^{i, \widehat{M}(i)} \prod_{i \in I_{N, L}} \widetilde{\mathcal{A}}_{L(i), N \circ L(i)}^{i, \widehat{N}(i),-\operatorname{sign}\left(V_{i}^{\prime}\right), \operatorname{sign}\left(V_{\widehat{N}(i)}^{\prime}\right)} \\
& \prod R_{M, N, L}\left(\left\langle\partial(w) \widetilde{\mathcal{B}}_{L\left(i_{0}\right), L\left(j_{0}\right)}^{i_{0}, j_{0}}\right\rangle_{i_{0}, j_{0}}(\phi)\right) .
\end{aligned}
$$

On souhaite comparer cette expression avec

$$
\mathcal{T}_{M_{1}, N, L_{1}}^{V_{a, b}, V_{i_{0}, j_{0}}^{\prime}}\left(\left\langle\partial(w) \widetilde{\mathcal{B}}_{L\left(i_{0}\right), L\left(j_{0}\right)}^{i_{0}, j_{0}}\right\rangle_{i_{0}, j_{0}}(\phi)\right)
$$

où les éléments $M_{1}$ et $L_{1}$ sont déduits de $M$ et $L$ (ils seront définis plus loin).

Pour $M, N \in \Omega(I \backslash\{a\}, J \backslash\{b\})$ disjoints, on notera les ensembles $I_{M, L}^{V_{a, b}, V_{i_{0}, j_{0}}^{\prime}}$, $I_{N, L}^{V_{a, b}, V_{i_{0}, j_{0}}^{\prime}}$ pour éviter l'ambiguïté avec les ensembles $I_{M, L}, I_{N, L}$; en effet, on a 
$\Omega(I \backslash\{a\}, J \backslash\{b\}) \subset \Omega(I, J)$. La relation d'équivalence sur les bijections $L, L^{\prime}$ de $\{1, \ldots, \mathrm{n}\} \backslash\left\{i_{0}, j_{0}\right\}$ dans $\{1, \ldots, \mathrm{n}\} \backslash\{a, b\}$ relative aux éléments $M$ et $N$ est notée $L \simeq_{M, N}^{V_{a, b}, V_{i_{0}, j_{0}}^{\prime}} L^{\prime}$. On a les équivalence suivantes pour des bijections $L, L^{\prime}$ : $\{1, \ldots, \mathrm{n}\} \backslash\left\{i_{0}, j_{0}\right\} \stackrel{\simeq}{\rightrightarrows}\{1, \ldots, \mathrm{n}\} \backslash\{a, b\}$

$$
\begin{aligned}
& L \simeq_{M, N}^{V_{a, b}, V_{i_{0}, j_{0}}^{\prime}} L^{\prime} \Longleftrightarrow L+\left[i_{0}, a\right]+\left[j_{0}, b\right] \simeq_{M+[a, b], N} L^{\prime}+\left[i_{0}, a\right]+\left[j_{0}, b\right], \\
& L \in \operatorname{Pr}^{V_{a, b}, V_{i_{0}, j_{0}}^{\prime}}(M, N) \Longleftrightarrow L+\left[i_{0}, a\right]+\left[j_{0}, b\right] \in \operatorname{Pr}(M+[a, b], N) .
\end{aligned}
$$

On obtient les relations suivantes entre ensembles :

$$
\begin{aligned}
I_{M+[a, b], L+\left[i_{0}, a\right]+\left[j_{0}, b\right]} & =I_{M, L}^{V_{a, b}, V_{i_{0}, j_{0}}^{\prime}} \amalg\left\{i_{0}\right\} \\
J_{M+[a, b], L+\left[i_{0}, a\right]+\left[j_{0}, b\right]} & =J_{M, L}^{V_{a}, V_{i_{0}, j_{0}}^{\prime}} \amalg\left\{j_{0}\right\} \\
I_{N, L+\left[i_{0}, a\right]+\left[j_{0}, b\right]} & =I_{N, L}^{V_{a, b}, V_{i_{0}, j_{0}}^{\prime}} \\
J_{N, L+\left[i_{0}, a\right]+\left[j_{0}, b\right]} & =J_{N, L}^{V_{a, b}, V_{i_{0}, j_{0}}^{\prime}} \\
I_{M+[a, b], N, L+\left[i_{0}, a\right]+\left[j_{0}, b\right]} & =I_{M,, N, V_{i_{0}, j_{0}}^{\prime}}^{V_{M, L}} \\
J_{M+[a, b], N, L+\left[i_{0}, a\right]+\left[j_{0}, b\right]} & =J_{M, N, L}^{V_{a, b}, V_{i_{0}, j_{0}}^{\prime}} .
\end{aligned}
$$

D'après ces relations, $\widehat{M}_{L}^{V_{a, b}, V_{i_{0}, j_{0}}^{\prime}}$ est la restriction de $\left.\widehat{M+[a}, b\right]_{L}$ à $I_{M, L}^{V_{a, b}, V_{i_{0}, j_{0}}^{\prime}}$ et $\widehat{N}_{L}^{V_{a, b}, V_{i_{0}, j_{0}}^{\prime}}$ est égal à $\widehat{N}_{L}$. L'égalité (5.1.14) associée aux sous-espaces $V_{a, b}$ et $V_{i_{0}, j_{0}}^{\prime}$ s'écrit

$$
\begin{aligned}
& \mathcal{T}_{M, N, \bar{L}_{M, N}^{V_{a, b}, V_{i_{0}, j_{0}}^{\prime}}}^{V_{a, V_{i}}^{\prime}, j_{0}}(\psi) \\
& =\varepsilon(L) \prod_{\substack{V_{a, b}, V_{i_{0}, j_{0}}^{\prime} \\
i \in I_{M, L}}} \widetilde{\mathcal{B}}_{L(i), M \circ L(i)}^{i, \widehat{M+[a, b}]_{L}(i)} \prod_{\substack{V_{a, b}, V_{i_{0}, j_{0}}^{\prime} \\
I_{N, L}}} \widetilde{\mathcal{A}}_{L(i), N \circ L(i)}^{i, \widehat{N}_{L}(i),-\operatorname{sign}\left(V_{i}^{\prime}\right), \operatorname{sign}\left(V_{\widehat{N}}^{\prime}(i)\right)} \prod R_{M, N, L}^{V_{a, b}, V_{i_{0}, j_{0}}^{\prime}}(\psi)
\end{aligned}
$$

où $\psi \in \widetilde{\mathcal{W}}_{c}\left(\mathfrak{h}_{V_{a, b}, M}, d, \Gamma_{N}\right)$ et $L \in \operatorname{Pr}^{V_{a, b}, V_{i_{0}, j_{0}}^{\prime}}(M, N)$. En comparant les applications linéaires $R_{M, N, L}^{V_{a, b}, V_{i_{0}, j_{0}}^{\prime}}$ et $R_{M+[a, b], N, L+\left[i_{0}, a\right]+\left[j_{0}, b\right]}$, d'après les relations (5.1.17), on a

$$
R_{M, N, L}^{V_{a, b}, V_{i_{0}, j_{0}}^{\prime}}=R_{M+[a, b], N, L+\left[i_{0}, a\right]+\left[j_{0}, b\right]},
$$

on en déduit l'égalité suivante

$$
\begin{aligned}
& \mathcal{T}_{M, N, \bar{L}_{M, N}^{V_{a, b}, V_{i_{0}, j_{0}}^{\prime}}}^{V_{a, b}, V_{i_{0}}^{\prime}}(\psi) \\
& =\varepsilon(L) \prod_{i \in I_{M, L}^{V_{a, b}, V_{i_{0}, j_{0}}^{\prime}}} \widetilde{\mathcal{B}}_{L(i), M \circ L(i)}^{i, \widehat{M+[a, b]}]_{L}(i)} \prod_{i \in I_{N, L+\left[i_{0}, a\right]+\left[j_{0}, b\right]}} \widetilde{\mathcal{A}}_{L(i), N \circ L(i)}^{i, \widehat{N}_{L}(i),-\operatorname{sign}\left(V_{i}^{\prime}\right), \operatorname{sign}\left(V_{\widehat{N}}^{\prime}(i)\right)} \\
& \prod R_{M+[a, b], N, L+\left[i_{0}, a\right]+\left[j_{0}, b\right]}(\psi) .
\end{aligned}
$$


On remarque ensuite que $\left\langle\partial(w) \widetilde{\mathcal{B}}_{M, a, b}^{i_{0}, j_{0}}\right\rangle_{i_{0}, j_{0}}(\phi) \in \widetilde{\mathcal{W}}_{c}\left(\mathfrak{h}_{V_{a, b}, M}, d, \Gamma_{N}\right)$ et d'après (5.1.17), on a $I_{M, L}^{V_{a, b}, V_{i_{0}, j_{0}}^{\prime}}=I_{M+[a, b], L+\left[i_{0}, a\right]+\left[j_{0}+a\right]}$. On en déduit l'égalité

$$
\begin{aligned}
\left\langle\partial(w) \mathcal{T}_{M+[a, b], N, \overline{L+\left[i_{0}, a\right]+\left[j_{0}, b\right]}}(\phi)\right\rangle_{i_{0}, j_{0}} & \\
= & \varepsilon\left(L+\left[i_{0}, a\right]+\left[j_{0}, b\right]\right) \varepsilon(L) \mathcal{T}_{M, N, \bar{L}_{M, N}^{V_{a}, V_{i_{0}, j_{0}}}}^{V_{a, b}, V_{i_{0}, j_{0}}^{\prime}}\left(\left\langle\partial(w) \widetilde{\mathcal{B}}_{a, b}^{i_{0}, j_{0}}\right\rangle_{i_{0}, j_{0}}(\phi)\right) .
\end{aligned}
$$

D'après le lemme 5.1.29, on a $\varepsilon\left(L+\left[i_{0}, a\right]+\left[j_{0}, b\right]\right) \varepsilon(L)=(-1)^{a+b+i_{0}+j_{0}}$, ainsi la relation précédente s'écrit

$$
\begin{aligned}
&\left\langle\partial(w) \mathcal{T}_{M+[a, b], N, \overline{L+\left[i_{0}, a\right]+\left[j_{0}, b\right]}}(\phi)\right\rangle_{i_{0}, j_{0}} \\
&=(-1)^{a+b+i_{0}+j_{0}} \mathcal{T}_{M, N, \bar{L}_{M, N}^{V_{a, b}, V_{i_{0}, j_{0}}}}^{V_{a, b}, V_{i_{0}, j_{0}}^{\prime}}\left(\left\langle\partial(w) \widetilde{\mathcal{B}}_{a, b}^{i_{0}, j_{0}}\right\rangle_{i_{0}, j_{0}}(\phi)\right) . \\
& \underbrace{}_{M,}
\end{aligned}
$$

$S i\left(i_{0}, j_{0}\right) \in \Gamma_{N, L}$, comme précédemment, on obtient

$$
\begin{aligned}
\left\langle\partial(w) \mathcal{T}_{M, N, L}\right\rangle_{i_{0}, j_{0}}=\varepsilon(L) & \prod_{i \in I_{M, L}} \widetilde{\mathcal{B}}_{L(i), M \circ L(i)}^{i, \widehat{M}(i)} \prod_{\substack{i \in I_{N, L} \\
i \neq i_{0}}} \widetilde{\mathcal{A}}_{L(i), N \circ L(i)}^{i, \widehat{N}(i),-\operatorname{sign}\left(V_{i}^{\prime}\right), \operatorname{sign}\left(V_{\widehat{N}(i)}^{\prime}\right)} \\
& \prod R_{M, N, L}\left(\left\langle\partial(w) \widetilde{\mathcal{A}}_{L\left(i_{0}\right), L\left(j_{0}\right)}^{i_{0}, j_{0},-\operatorname{sign}\left(V_{i_{0}}^{\prime}\right), \operatorname{sign}\left(V_{j_{0}}^{\prime}\right)}\right\rangle_{i_{0}, j_{0}}(\phi)\right) .
\end{aligned}
$$

On procède ensuite comme dans le cas précédent et on utilise les mêmes notations. On a les équivalences suivantes pour deux bijections $L, L^{\prime}:\{1, \ldots, \mathrm{n}\} \backslash\left\{i_{0}, j_{0}\right\} \stackrel{\simeq}{\rightrightarrows}$ $\{1, \ldots, \mathrm{n}\} \backslash\{a, b\}$

$$
L \simeq_{M, N}^{V_{a, b}, V_{i_{0}, j_{0}}^{\prime}} L^{\prime} \Longleftrightarrow L+\left[i_{0}, a\right]+\left[j_{0}, b\right] \simeq_{M, N+[a, b]} L^{\prime}+\left[i_{0}, a\right]+\left[j_{0}, b\right]
$$

et

$$
L \in \operatorname{Pr}^{V_{a, b}, V_{i_{0}, j_{0}}^{\prime}}(M, N) \Longleftrightarrow L+\left[i_{0}, a\right]+\left[j_{0}, b\right] \in \operatorname{Pr}(M, N+[a, b]) .
$$

Puis, on obtient les égalités suivantes entre ensembles

$$
\begin{aligned}
I_{M, L+\left[i_{0}, a\right]+\left[j_{0}, b\right]} & =I_{M, L}^{V_{a, b}, V_{i_{0}, j_{0}}^{\prime}} \\
J_{M, L+\left[i_{0}, a\right]+\left[j_{0}, b\right]} & =J_{M, L}^{V_{a, b}, V_{i_{0}, j_{0}}^{\prime}} \\
I_{N+[a, b], L+\left[i_{0}, a\right]+\left[j_{0}, b\right]} & =I_{N, L}^{V_{a}, V_{i_{0}, j_{0}}^{\prime}} \amalg\left\{i_{0}\right\} \\
J_{N+[a, b], L+\left[i_{0}, a\right]+\left[j_{0}, b\right]} & =J_{N, L}^{V_{a, b}, V_{i_{0}, j_{0}}^{\prime}} \amalg\left\{j_{0}\right\} \\
I_{M, N+[a, b], L+\left[i_{0}, a\right]+\left[j_{0}, b\right]} & =I_{M, b, V_{i_{0}, j_{0}}^{\prime}}^{V_{a, L}} \\
J_{M, N+[a, b], L+\left[i_{0}, a\right]+\left[j_{0}, b\right]} & =J_{M, N, L}^{V_{a, b}, V_{i_{0}, j_{0}}^{\prime}}
\end{aligned}
$$


D'après ces relations, $\widehat{N}_{L}^{V_{a, b}, V_{i_{0}, j_{0}}^{\prime}}$ est la restriction de $\widehat{N}_{L}$ à $I_{M, N, L}^{V_{a, b}, V_{i_{0}, j_{0}}^{\prime}}$ et $\widehat{M}_{L}^{V_{a, b}, V_{i_{0}, j_{0}}^{\prime}}=\widehat{M}_{L}$. On en déduit la relation

$$
\begin{aligned}
& \left\langle\partial(w) \mathcal{T}_{M, N+[a, b], \overline{L+\left[i_{0}, a\right]+\left[j_{0}, b\right]}}(\phi)\right\rangle_{i_{0}, j_{0}} \\
& =(-1)^{a+b+i_{0}+j_{0}} \mathcal{T}^{V_{a, b}, V_{i_{0}, j_{0}}^{\prime}}\left(\left\langle\partial(w) \widetilde{\mathcal{A}}_{a, b}^{i_{0}, j_{0},-\operatorname{sign}\left(V_{i_{0}}^{\prime}\right), \operatorname{sign}\left(V_{j_{0}}^{\prime}\right)}\right\rangle_{i_{0}, j_{0}}(\phi)\right),
\end{aligned}
$$

en utilisant à nouveau le lemme 5.1.29.

On peut à présent en déduire le saut de $\mathcal{R}_{M}(\phi)\left(x^{\prime}\right)$. On fixe $N \in \Omega$ disjoint avec $M$. On rappelle que $\operatorname{Pr}(M, N)$ est en bijection canonique avec les classes d'équivalence de $\simeq_{M, N}$. Par définition de $\mathcal{R}_{M}$, on a

$$
\begin{aligned}
& \left\langle\partial(w) \mathcal{R}_{M}(\phi)\left(x^{\prime}\right)\right\rangle_{i_{0}, j_{0}}=\sum_{L \in \operatorname{Pr}(M, N)}\left\langle\partial(w) \mathcal{T}_{M, N, L}\right\rangle_{i_{0}, j_{0}} \\
& =\sum_{\substack{L \in \operatorname{Pr}(M, N) \\
\operatorname{avec}\left(i_{0}, j_{0}\right) \in \Gamma_{M, L}}}\left\langle\partial(w) \mathcal{T}_{M, N, L}(\phi)\right\rangle_{i_{0}, j_{0}}+\sum_{\begin{array}{c}
L \in \operatorname{Pr}(M, N) \\
\operatorname{avec}\left(i_{0}, j_{0}\right) \in \Gamma_{N, L}
\end{array}}\left\langle\partial(w) \mathcal{T}_{M, N, L}(\phi)\right\rangle_{i_{0}, j_{0}} \\
& =\sum_{\substack{L \in \operatorname{Pr}(M, N) \\
\text { avec }\left(i_{0}, j_{0}\right) \in \Gamma_{M, L}}}(-1)^{i_{0}+j_{0}+L\left(i_{0}\right)+L\left(j_{0}\right)} \mathcal{T}_{M_{1}, N, L_{1}}^{V_{L\left(i_{0}\right), L\left(j_{0}\right)}, V_{i_{0}, j_{0}}^{\prime}}\left(\left\langle\partial(w) \widetilde{\mathcal{B}}_{L\left(i_{0}\right), L\left(j_{0}\right)}^{i_{0}, j_{0}}(\phi)\right\rangle_{i_{0}, j_{0}}\right) \\
& +\sum_{\substack{L \in \operatorname{Pr}(M, N) \\
\operatorname{avec}\left(i_{0}, j_{0}\right) \in \Gamma_{N, L}}}(-1)^{i_{0}+j_{0}+L\left(i_{0}\right)+L\left(j_{0}\right)} \mathcal{T}_{M, N_{1}, L_{1}}^{V_{L\left(i_{0}\right), L\left(j_{0}\right)}, V_{i_{0}, j_{0}}^{\prime}} \\
& \left.\qquad\left\langle\partial(w) \widetilde{\mathcal{A}}_{L\left(i_{0}\right), L\left(j_{0}\right)}^{i_{0}, j_{0},-\operatorname{sign}\left(V_{i_{0}}^{\prime}\right), \operatorname{sign}\left(V_{j_{0}}^{\prime}\right)}(\phi)\right\rangle_{i_{0}, j_{0}}\right)
\end{aligned}
$$

où l'application $M_{1}$ (resp. $\left.N_{1}, L_{1}\right)$ est la restriction de $M($ resp. $N, L)$ à $\mathrm{A}_{M} \backslash\left\{L\left(i_{0}\right)\right\}$ (resp. $\left.\mathrm{A}_{N} \backslash\left\{L\left(i_{0}\right)\right\},\{1, \ldots, n\} \backslash\left\{L\left(i_{0}\right), L\left(j_{0}\right)\right\}\right)$. On a les égalités suivantes

$$
\begin{aligned}
& \sum_{\substack{L \in \operatorname{Pr}(M, N) \\
\text { avec }\left(i_{0}, j_{0}\right) \in \Gamma_{M, L}}}(-1)^{i_{0}+j_{0}+L\left(i_{0}\right)+L\left(j_{0}\right)} \mathcal{T}_{M_{1}, N, L_{1}}^{V_{L\left(i_{0}\right), L\left(j_{0}\right)}, V_{i_{0}, j_{0}}^{\prime}}\left(\left\langle\partial(w) \widetilde{\mathcal{B}}_{L\left(i_{0}\right), L\left(j_{0}\right)}^{i_{0}, j_{0}}(\phi)\right\rangle_{i_{0}, j_{0}}\right) \\
= & \sum_{a \in \mathbb{A}_{M}}(-1)^{i_{0}+j_{0}+a+M(a)} \sum_{L \in \operatorname{Pr}^{V} V_{a, M(a)}, v_{i_{0}, j_{0}}^{\prime}\left(M_{1}, N\right)} \mathcal{T}_{M_{1}, N, L}^{V_{a, M(a)}, V_{i_{0}, j_{0}}^{\prime}}\left(\left\langle\partial(w) \widetilde{\mathcal{B}}_{a, M(a)}^{i_{0}, j_{0}}(\phi)\right\rangle_{i_{0}, j_{0}}\right) \\
= & \sum_{a \in \mathbb{A}_{M}}(-1)^{i_{0}+j_{0}+a+M(a)} \mathcal{R}_{M_{1}}^{V_{a, M(a)}, V_{i_{0}, j_{0}}^{\prime}}\left(\left\langle\partial(w) \widetilde{\mathcal{B}}_{a, M(a)}^{i_{0}, j_{0}}(\phi)\right\rangle_{i_{0}, j_{0}}\right) .
\end{aligned}
$$

De même, on a

$$
\begin{aligned}
\sum_{\substack{L \in \operatorname{Pr}(M, N) \\
\operatorname{avec}\left(i_{0}, j_{0}\right) \in \Gamma_{N, L}}}(-1)^{i_{0}+j_{0}+L\left(i_{0}\right)+L\left(j_{0}\right)} \mathcal{T}_{M, N_{1}, L_{1}}^{V_{L\left(i_{0}\right), L\left(j_{0}\right)}, V_{i_{0}, j_{0}}^{\prime}} & \left(\left\langle\partial(w) \widetilde{\mathcal{A}}_{L\left(i_{0}\right), L\left(j_{0}\right)}^{i_{0}, j_{0},-\operatorname{sign}\left(V_{i_{0}}^{\prime}\right), \operatorname{sign}\left(V_{j_{0}}^{\prime}\right)}(\phi)\right\rangle_{i_{0}, j_{0}}\right) \\
& \left(\langle)^{\prime}\right)
\end{aligned}
$$




$$
\begin{aligned}
=\sum_{a \in \mathrm{A}_{N}}(-1)^{i_{0}+j_{0}+a+N(a)} \sum_{L \in \operatorname{Pr}{ }_{a, N(a)}, V_{i_{0}, j_{0}}^{\prime}\left(M, N_{1}\right)} \mathcal{T}_{M, N_{1}, L}^{V_{a, N(a)}, V_{i_{0}, j_{0}}^{\prime}} \\
\left(\left\langle\partial(w) \widetilde{\mathcal{A}}_{a, N(a)}^{i_{0}, j_{0},-\operatorname{sign}\left(V_{i_{0}}^{\prime}\right), \operatorname{sign}\left(V_{j_{0}}^{\prime}\right)}(\phi)\right\rangle_{i_{0}, j_{0}}\right) \\
=\sum_{a \in \mathrm{A}_{N}}(-1)^{i_{0}+j_{0}+a+N(a)} \mathcal{R}_{M}^{V_{a, N(a)}, V_{i_{0}, j_{0}}^{\prime}} \\
\left(\left\langle\partial(w) \tilde{\mathcal{A}}_{a, N(a)}^{i_{0}, j_{0},-\operatorname{sign}\left(V_{i_{0}}^{\prime}\right), \operatorname{sign}\left(V_{j_{0}}^{\prime}\right)}(\phi)\right\rangle_{i_{0}, j_{0}}\right) .
\end{aligned}
$$

L'expression (5.1.18) devient

$$
\begin{aligned}
& \left\langle\partial(w) \mathcal{R}_{M}(\phi)\left(x^{\prime}\right)\right\rangle_{i_{0}, j_{0}} \\
& =\sum_{a \in \mathrm{A}_{M}}(-1)^{i_{0}+j_{0}+a+M(a)} \mathcal{R}_{M_{1}}^{V_{a, M(a)}, V_{i_{0}, j_{0}}^{\prime}}\left(\left\langle\partial(w) \widetilde{\mathcal{B}}_{a, M(a)}^{i_{0}, j_{0}}(\phi)\right\rangle_{i_{0}, j_{0}}\right) \\
& +\sum_{a \in \mathrm{A}_{N}}(-1)^{i_{0}+j_{0}+a+N(a)} \mathcal{R}_{M}^{V_{a, N(a)}, V_{i_{0}, j_{0}}^{\prime}} \\
& \left(\left\langle\partial(w) \widetilde{\mathcal{A}}_{a, N(a)}^{i_{0}, j_{0},-\operatorname{sign}\left(V_{i_{0}}^{\prime}\right), \operatorname{sign}\left(V_{j_{0}}^{\prime}\right)}(\phi)\right\rangle_{i_{0}, j_{0}}\right) .
\end{aligned}
$$

Comme $\phi \in \widetilde{\mathcal{W}}_{c}\left(\mathfrak{h}_{M}, d, \Gamma_{N}\right)$, pour $(a, b) \in I_{M} \times J_{M}$ tel que $b \neq N(a)$, on a d'après le lemme 5.1.28

$$
\left\langle\partial(w) \widetilde{\mathcal{A}}_{a, b}^{i_{0}, j_{0},-\operatorname{sign}\left(V_{i_{0}}^{\prime}\right), \operatorname{sign}\left(V_{j_{0}}^{\prime}\right)}(\phi)\right\rangle_{i_{0}, j_{0}}=0 .
$$

Cela permet d'écrire la relation (5.1.19) ainsi

$$
\begin{aligned}
& \left\langle\partial(w) \mathcal{R}_{M}(\phi)\left(x^{\prime}\right)\right\rangle_{i_{0}, j_{0}} \\
& =\sum_{a \in A_{M}}(-1)^{i_{0}+j_{0}+a+M(a)} \mathcal{R}_{M_{1}}^{V_{a, M(a)}, V_{i_{0}, j_{0}}^{\prime}}\left(\left\langle\partial(w) \widetilde{\mathcal{B}}_{a, M(a)}^{i_{0}, j_{0}}(\phi)\right\rangle_{i_{0}, j_{0}}\right) \\
& +\sum_{(a, b) \in I_{M} \times J_{M}}(-1)^{i_{0}+j_{0}+a+b} \mathcal{R}_{M}^{V_{a, b}, V_{i_{0}, j_{0}}^{\prime}}\left(\left\langle\partial(w) \widetilde{\mathcal{A}}_{a, b}^{i_{0}, j_{0},-\operatorname{sign}\left(V_{i_{0}}^{\prime}\right), \operatorname{sign}\left(V_{j_{0}}^{\prime}\right)}(\phi)\right\rangle_{i_{0}, j_{0}}\right) .
\end{aligned}
$$

Comme cette relation est indépendante de $N$ et vérifiée pour tout $N$ disjoint de $M$, d'après le théorème 4.3 .11 , elle est vérifiée pour $\phi \in \widetilde{\mathcal{W}}_{c}\left(\mathfrak{h}_{M}, d, I_{M} \times J_{M}\right)$.

Pour une injection $L$ d'une partie $P$ de $\mathbb{N}$ dans $\mathbb{N}$, on note

$$
\varepsilon(L)=(-1)^{\operatorname{Card}\left\{(i, j) \in P^{2} \mid i<j \text { et } L(i)>L(j)\right\}} .
$$

Pour $L \in \mathfrak{S}(P)$, on retrouve la définition de la signature de $L$.

Lemme 5.1.29. - Soient $L$ une bijection de $\{1, \ldots, \mathrm{n}\}$ dans $\{1, \ldots, \mathrm{n}\}$ et $i_{0}, a \in$ $\{1, \ldots, \mathrm{n}\}$ tels que $L\left(i_{0}\right)=a$ On note $L_{1}$ la restriction de $L \grave{a}\{1, \ldots, \mathrm{n}\} \backslash\left\{i_{0}\right\}$. On a alors la relation:

$$
\varepsilon(L)=\varepsilon\left(L_{1}\right)(-1)^{a+i_{0}} .
$$


Démonstration. - On rappelle la définition suivante :

$$
\varepsilon\left(L_{1}\right)=(-1)^{\operatorname{Card}\left\{(i, j) \in\{1, \ldots, n\} \backslash\left\{i_{0}\right\}^{2} \mid i<j \text { et } L_{1}(i)>L_{1}(j)\right\}}
$$

On a les relations modulo 2 suivantes sur les cardinaux :

$$
\begin{aligned}
& \operatorname{Card}\left\{i<i_{0} \mid L(i)>a\right\}+\operatorname{Card}\left\{i>i_{0} \mid L(i)<a\right\} \\
& =\operatorname{Card}\{i \mid L(i)>a\}+\operatorname{Card}\left\{i>i_{0} \mid L(i)>a\right\}+\operatorname{Card}\left\{i>i_{0} \mid L(i)>a\right\} \\
& +\operatorname{Card}\left\{i>i_{0}\right\} \\
& =a+i_{0} .
\end{aligned}
$$

On en déduit le résultat.

Pour le prochain théorème, on a besoin d'introduire les notations suivantes :

Soient $\phi \in \mathcal{D}(\mathfrak{g})$ et $M, N \in \Omega$ disjoints. On rappelle que

$$
V=\underset{i \in\{1, \ldots, \mathrm{n}\}}{\stackrel{\perp}{\oplus}} \mathbb{C} f_{i} \text { avec }\left(f_{i}, f_{i}\right)= \begin{cases}1 & \text { si } i \leqslant \mathrm{p} ; \\ -1 & \text { si } i>\mathrm{p} .\end{cases}
$$

On considère les sous-espaces de $V$

$$
U_{N}=\underset{i \notin \mathrm{A}_{N} \cup \operatorname{im}(N)}{\stackrel{1}{\oplus}} \mathbb{C} f_{i}, \quad X_{N}=\underset{i \in \mathrm{A}_{N}}{\oplus} \mathbb{C}\left(f_{i}-f_{N(i)}\right), \quad Y_{N}=\underset{i \in \mathrm{A}_{N}}{\oplus} \mathbb{C}\left(f_{i}+f_{N(i)}\right) .
$$

On note $\mathfrak{p}_{N}$ la sous-algèbre parabolique de $\mathfrak{g}$

$$
\mathfrak{p}_{N}=\left\{u \in \mathfrak{g} \mid u\left(X_{N}\right) \subset X_{N}\right\}
$$

et $\mathfrak{n}_{N}$ le radical unipotent de $\mathfrak{p}_{N}$. La restriction du produit hermitien $($,$) à U_{N}$ est non dégénérée. On considère la sous-algèbre de Lie de $\mathfrak{g}$

$$
\mathfrak{g}\left(U_{N}\right)=\left\{u \in \mathfrak{g l}_{\mathbb{C}}\left(U_{N}\right) \mid(u(x), y)+(x, u(y))=0 \forall x, y \in U_{N}\right\} .
$$

Les espaces $X_{N}$ et $Y_{N}$ sont totalement isotropes; de plus la restriction de (, ) à $X_{N} \oplus Y_{N}$ est non dégénérée. L'isomorphisme d'espaces vectoriels

$$
\begin{aligned}
\theta_{N}: X_{N} & \longrightarrow Y_{N} \\
f_{i}-f_{N(i)} & \longmapsto f_{i}+f_{N(i)}
\end{aligned}
$$

est tel que la forme bilinéaire

$$
\begin{aligned}
(,)_{N}: X_{N} \times X_{N} & \longrightarrow \mathbb{C} \\
(x, y) & \longmapsto\left(x, \theta_{N}(y)\right)
\end{aligned}
$$

est un produit scalaire sur $X_{N}$. Pour $u \in \mathfrak{g l}_{\mathbb{C}}\left(X_{N}\right)$, on note $u^{*} \in \mathfrak{g l}_{\mathbb{C}}\left(X_{N}\right)$ l'adjoint de $u$ défini par

$$
(u(x), y)_{N}=\left(x, u^{*}(y)\right)_{N} \forall x, y \in X_{N}
$$

On montre alors que l'application $\mathbb{R}$-linéaire

$$
\begin{aligned}
\mathfrak{g l}_{\mathbb{C}}\left(X_{N}\right) & \longrightarrow \mathfrak{g l}_{\mathbb{C}}\left(X_{N}\right) \oplus \mathfrak{g l}_{\mathbb{C}}\left(Y_{N}\right) \\
u & \longmapsto u \oplus-\theta_{N} \circ u^{*} \circ \theta_{N}^{-1}
\end{aligned}
$$


définit une injection de $\mathfrak{g l}_{\mathbb{C}}\left(X_{N}\right)$ dans $\mathfrak{p}_{N}$. On identifie ainsi $\mathfrak{g l}_{\mathbb{C}}\left(X_{N}\right)$ à une sousalgèbre de $\mathfrak{p}_{N}$ et $G L\left(X_{N}\right)$ à un sous-groupe de $G$. La restriction de la forme $\kappa_{\mathfrak{g}}$ aux espaces $\mathfrak{g}\left(U_{N}\right)$ et $\mathfrak{g l}_{\mathbb{C}}\left(X_{N}\right)$ est non dégénérée. Cela permet de considérer les mesures $d g_{1}$ et $d g_{2}$ respectivement sur $G\left(U_{N}\right)$ et $G L\left(X_{N}\right)$. On observe que la sous-algèbre de Cartan $\mathfrak{h}_{M+N}$ de $\mathfrak{g}$ stabilise $X_{N}$ et $Y_{N}$. Elle se décompose naturellement sous la forme

$$
\mathfrak{h}_{M+N}=\mathfrak{h}_{U_{N}, M} \oplus \mathfrak{h}_{X_{N}}
$$

où $\mathfrak{h}_{U_{N}, M} \in \operatorname{Car}\left(\mathfrak{g}\left(U_{N}\right)\right)$ et $M$ s'identifie à un système de racines fortement orthogonales pour cette sous-algèbre; de même $\mathfrak{h}_{X_{N}} \in \operatorname{Car}\left(\mathfrak{g l}_{\mathbb{C}}\left(X_{N}\right)\right)$. La sous-algèbre $\mathfrak{g}\left(U_{N}\right) \oplus \mathfrak{g l}_{\mathbb{C}}\left(X_{N}\right)$ est un facteur de Levi de $\mathfrak{p}_{N}$, que l'on note $\mathfrak{l}_{N}$. Il existe une unique mesure $d n$ sur $\mathfrak{n}_{N}$ telle que

$$
\begin{aligned}
\left|\operatorname{det}\left(\operatorname{ad}\left(x_{1}+x_{2}\right) \mid \mathfrak{n}_{N}\right)\right| \int_{G / H_{M+N}} \phi\left(g \cdot\left(x_{1}+x_{2}\right)\right) d \dot{g} & \\
\quad= & \int_{G\left(U_{N}\right) / H_{U_{N}, M}} \int_{G L_{\mathbb{C}}\left(X_{N}\right) / H_{X_{N}}} \phi_{\mathfrak{n}_{N}}^{K}\left(g_{1} \cdot x_{1}+g_{2} \cdot x_{2}\right) d \dot{g_{1}} d \dot{g_{2} .} .
\end{aligned}
$$

où

$$
\phi_{\mathfrak{n}_{N}}^{K}(x)=\int_{K \times \mathfrak{n}_{N}} \phi(k \cdot(x+n)) d k d n
$$

pour $x \in \mathfrak{l}_{N}$. L'ensemble des racines de $\mathfrak{h}_{U_{N}, M}$ positives en tant que racines de $\mathfrak{h}_{M+N}$ constitue un système de racines positives pour $\mathfrak{h}_{U_{N}, M}$ que l'on note $\Phi\left(\mathfrak{h}_{U_{N}, M}\right)$. De même, on note $\Phi\left(\mathfrak{h}_{X_{N}}\right)$ l'ensemble des racines de $\mathfrak{h}_{X_{N}}$ positives en tant que racines de $\mathfrak{h}_{M+N}$. Pour une fonction $\phi \in \mathcal{D}(\mathfrak{g})$, on rappelle la définition de l'intégrale invariante de Harish-Chandra de $\phi$ définie sur $\mathfrak{h}_{M+N}^{\text {reg }}$ :

$$
\phi_{M+N}(x)=\overline{\pi_{\mathfrak{h} \varnothing}}\left(c_{M+N}^{-1}(x)\right) \mathbf{e}_{M+N}(x) \int_{G / H_{M+N}} \phi\left(g \cdot\left(x_{1}+x_{2}\right)\right) d \dot{g} .
$$

De même, pour $\psi \in \mathcal{D}\left(\mathfrak{g}\left(U_{N}\right)\right)$, l'intégrale invariante de Harish-Chandra de $\psi$ sur $\mathfrak{h}_{U_{N}, M}^{\text {reg }}$ est définie par

$$
\phi_{M}^{U_{N}}(x)=\overline{\pi_{\mathfrak{h}_{N}, \varnothing}}\left(c_{M}^{-1}(x)\right) \mathbf{e}_{M}(x) \int_{G\left(U_{N}\right) / H_{U_{N}, M}} \phi\left(g \cdot\left(x_{1}+x_{2}\right)\right) d \dot{g}
$$

où

$$
\pi_{\mathfrak{h}_{U_{N}, \varnothing}}(x)=\prod_{\alpha \in \Phi\left(\mathfrak{h}_{\left.U_{N}, \varnothing\right)}\right.} \alpha(x)
$$

Et enfin, pour une fonction $\psi \in \mathcal{D}\left(\mathfrak{g l}_{\mathbb{C}}\left(X_{N}\right)\right)$, on pose pour $x \in \mathfrak{h}_{X_{N}}^{\text {reg }}$

où

$$
\psi^{G L\left(X_{N}\right)}(x)=D_{X_{N}}(x) \int_{G L\left(X_{N}\right) / H_{X_{N}}} \psi(g \cdot x) d \dot{g}
$$

$$
D_{X_{N}}(x)=\left|\prod_{\alpha \in \Phi\left(\mathfrak{h}_{X_{N}}\right)} \alpha(x)\right| .
$$


Pour $x \in \mathfrak{h}_{M+N}^{\mathrm{reg}}$, on pose

$$
\mathbf{e}_{N}(x)=\operatorname{sign}\left(\prod_{\alpha \in \mathcal{S}_{N}} \bar{\alpha}(x)\right) .
$$

ThÉorème 5.1.30. - Pour $\phi \in \mathcal{D}(\mathfrak{g}), w \in \operatorname{Sym}\left(\mathfrak{h}_{\varnothing, \mathbb{C}}^{\prime}\right)$ et $\left(i_{0}, j_{0}\right) \in\{1, \ldots, \mathrm{n}\}^{2}$ avec $i_{0}<j_{0}$, on $a$

$$
\left\langle\partial(w) \operatorname{Chc}(\phi)_{\varnothing}\right\rangle_{i_{0}, j_{0}}\left(x^{\prime}\right)=0 \text { si } \alpha=e_{i_{0}}-e_{j_{0}} \text { est une racine compacte }
$$

et

$$
\begin{aligned}
\left\langle\partial(w) \operatorname{Chc}(\phi)_{\varnothing}\right\rangle_{i_{0}, j_{0}}( & \left.x^{\prime}\right)=\mathrm{i} \pi^{2}(-1)^{i_{0}+j_{0}+\mathrm{n}} 16^{\mathrm{n}-1} \\
& \mathbf{C h c}^{V_{\mathrm{p}, \mathrm{n}}, V_{i_{0}, j_{0}}^{\prime}}\left(\partial\left(c_{\mathrm{p}, \mathrm{n}} t r_{i_{0}, j_{0}}^{\mathrm{p}, \mathrm{n}}(w)\right)\left(\phi_{\mathfrak{n}_{\mathrm{p}, \mathrm{n}}}^{K}\right)^{G L\left(X_{[\mathrm{p}, \mathrm{n}]}\right)}\left(v^{\prime}\right)\right)_{\varnothing}\left(y^{\prime}\right)
\end{aligned}
$$

si $\alpha$ est une racine non compacte pour $x^{\prime} \in \mathfrak{h}_{\varnothing}^{\prime\left(i_{0}, j_{0}\right)}$ où $y^{\prime}$ (resp. $\left.v^{\prime}\right)$ désigne la composante dans $\mathfrak{h}_{V_{i_{0}, j_{0}}, \varnothing}^{\prime}$ (resp. $\left.\mathbb{R}\left(\mathrm{i} E_{i_{0}, i_{0}}+\mathrm{i} E_{j_{0}, j_{0}}\right)\right)$ de $x^{\prime}$ par rapport à la décomposition

$$
\left\{x \in \mathfrak{h}_{\varnothing}^{\prime} \mid \alpha(x)=0\right\}=\mathfrak{h}_{V_{i_{0}, j_{0}}, \varnothing} \oplus \mathbb{R}\left(\mathrm{i} E_{i_{0}, i_{0}}+\mathrm{i} E_{j_{0}, j_{0}}\right) .
$$

La démonstration de ce théorème nécessite les lemmes suivants :

Lemme 5.1.31. - Pour $\phi \in \mathcal{D}(\mathfrak{g})$ et $M, N \in \Omega$ disjoints, on a pour $x \in \mathfrak{h}_{M+N}^{\text {reg }}$

$$
\phi_{M+N}(x)=\varepsilon(N) \mu(N)\left(\left(\phi_{\mathfrak{n}_{N}}^{K}\right)_{M}^{U_{N}}\right)^{G L\left(X_{N}\right)} ;
$$

où

$$
\mu(N)=(-1)^{\operatorname{Card}\left\{(i, a) \in I \times \mathbf{A}_{N} \mid i<a\right\}+\operatorname{Card}\left\{(j, a) \in J \times \mathbf{A}_{N} \mid j>N(a)\right\}+\frac{\operatorname{Card}\left(\mathrm{A}_{N}\right)\left(\operatorname{Card}\left(\mathrm{A}_{N}\right)-1\right)}{2} .}
$$

Dans le cas particulier où $N=[a, b]$, on a le résultat suivant

Corollaire 5.1.32. - Pour $N=[a, b]$, avec $(a, b) \in I_{M} \times J_{M}$, on a pour $x \in$ $\mathfrak{h}_{M+[a, b]}^{\text {reg }}$

$$
\phi_{M+[a, b]}=(-1)^{a+b+n}\left(\left(\phi_{\mathfrak{n}_{[a, b]}}^{K}\right)^{G L\left(X_{[a, b]}\right)}\right)_{M}^{U_{[a, b]}} .
$$

Démonstration du lemme 5.1.31. - Pour $x_{1} \in \mathfrak{h}_{U_{N}, M}$ et $x_{2} \in \mathfrak{h}_{X_{N}}$ tel que $x_{1}+x_{2} \in$ $\mathfrak{h}_{M+N}^{\text {reg }}$, on a

$$
\begin{aligned}
\left|\operatorname{det}\left(\operatorname{ad}\left(x_{1}+x_{2}\right) \mid \mathfrak{n}_{N}\right)\right| & \int_{G / H_{M+N}} \phi\left(g \cdot\left(x_{1}+x_{2}\right)\right) d \dot{g} \\
& =\int_{G\left(U_{N}\right) / H_{U_{N}, M}} \int_{G L_{\mathbb{C}}\left(X_{N}\right) / H_{X_{N}}} \phi_{\mathfrak{n}_{N}}^{K}\left(g_{1} \cdot x_{1}+g_{2} \cdot x_{2}\right) d \dot{g_{1}} d \dot{g_{2}} .
\end{aligned}
$$


Soit $E$ l'ensemble des racines $\alpha \in \Phi\left(\mathfrak{h}_{M+N}\right)$ (système de racines positives) qui ne sont pas des racines de $\mathfrak{h}_{U_{N}, M}$. On remarque que $\mathbf{e}_{M+N}(x)=\mathbf{e}_{U_{N}, M}\left(x_{1}\right) \mathbf{e}_{N}(x)$. La relation (5.1.21) permet d'obtenir

$$
\begin{aligned}
& \phi_{M+N}\left(x_{1}+x_{2}\right) \\
& \quad=\mathbf{e}_{N}(x) \frac{\left.\prod_{\alpha \in E} \bar{\alpha}\left(x_{1}+x_{2}\right)\right)}{D_{X_{N}}\left(x_{2}\right)\left|\operatorname{det}\left(\left.\operatorname{ad}\left(x_{1}+x_{2}\right)\right|_{\mathfrak{n}_{N}}\right)\right|}\left(\left(\phi_{\mathfrak{n}_{N}}^{K}\right)^{G L\left(X_{N}\right)}\right)_{M}^{U N}\left(x_{1}+x_{2}\right) .
\end{aligned}
$$

On montre que la transformée de Cayley $c_{M+N}$ vérifie pour $j \in\{1, \ldots, \mathrm{n}\}$

$$
\overline{e_{j}}\left(c_{M+N}^{-1}(x)\right)= \begin{cases}-u_{j}-\mathrm{i} v_{j} & \text { si } j \in \mathrm{A}_{M+N} \\ u_{(M+N)^{-1}(j)}-\mathrm{i} v_{(M+N)^{-1}(j)} & \text { si } j \in \operatorname{im}(M+N) \\ -\mathrm{i} x_{j} & \text { sinon }\end{cases}
$$

Dans la suite de cette démonstration, pour deux parties finies $\mathcal{Q}_{1}, \mathcal{Q}_{2}$ de $\mathbb{N}$, on pose

$$
\prec \mathcal{Q}_{1}, \mathcal{Q}_{2} \succ=\operatorname{Card}\left\{(a, b) \in \mathcal{Q}_{1} \times \mathcal{Q}_{2} \mid a<b\right\} \text { modulo } 2
$$

Pour $j \in \mathrm{A}_{M}$ et $a \in \mathrm{A}_{N}$, on a

$$
\begin{aligned}
& \overline{e_{a}-e_{M(j)}}\left(c_{M+N}^{-1}(x)\right)=-u_{a}-\mathrm{i} v_{a}-u_{j}+\mathrm{i} v_{j} \\
& \overline{e_{j}-e_{N(a)}}\left(c_{M+N}^{-1}(x)\right)=-u_{a}+\mathrm{i} v_{a}-u_{j}-\mathrm{i} v_{j}
\end{aligned}
$$

ainsi

$$
\overline{e_{a}-e_{M(j)}}\left(c_{M+N}^{-1}(x)\right) \overline{e_{j}-e_{N(a)}}\left(c_{M+N}^{-1}(x)\right)>0
$$

et

$$
\operatorname{sign}\left\{\prod_{\substack{j \in A_{M} \\ a \in \mathrm{A}_{N}}}\left(\overline{e_{a}-e_{M(j)}}\left(c_{M+N}^{-1}(x)\right) \overline{e_{j}-e_{N(a)}}\left(c_{M+N}^{-1}(x)\right)\right)\right\}=1 .
$$

On obtient aussi

$$
\operatorname{sign}\left\{\prod_{\substack{a, j \in \mathrm{A}_{N} \\ a<j}}\left(\overline{e_{a}-e_{N(j)}}\left(c_{M+N}^{-1}(x)\right) \overline{e_{j}-e_{N(a)}}\left(c_{M+N}^{-1}(x)\right)\right)\right\}=1 .
$$

Par définition, on a

$$
\operatorname{sign}\left\{\prod_{a \in \mathrm{A}_{N}} \overline{e_{a}-e_{N(a)}}\left(c_{M+N}^{-1}(x)\right)\right\}=e_{N}(x) .
$$

Pour $j \in \mathrm{A}_{M}$ et $a \in \mathrm{A}_{N}$, on a

$$
\begin{aligned}
\overline{e_{j}-e_{a}}\left(c_{M+N}^{-1}(x)\right) & =-u_{j}-\mathrm{i} v_{j}+u_{a}+\mathrm{i} v_{a} \\
\overline{e_{M(j)}-e_{N(a)}}\left(c_{M+N}^{-1}(x)\right) & =u_{j}-\mathrm{i} v_{j}-u_{a}+\mathrm{i} v_{a}
\end{aligned}
$$

ainsi

$$
\overline{e_{j}-e_{a}}\left(c_{M+N}^{-1}(x)\right) \overline{e_{M(j)}-e_{N(a)}}\left(c_{M+N}^{-1}(x)\right)<0
$$


et

$$
\begin{aligned}
& \operatorname{sign}\left\{\prod_{\substack{j \in \mathrm{A}_{M}, a \in \mathrm{A}_{N} \\
j<a, M(j)<N(a)}}\left(\overline{e_{j}-e_{a}}\left(c_{M+N}^{-1}(x)\right) \overline{e_{M(j)}-e_{N(a)}}\left(c_{M+N}^{-1}(x)\right)\right)\right. \\
& \prod_{\substack{j \in \mathrm{A}_{M}, a \in \mathrm{A}_{N} \\
j>a, M(j)<N(a)}}\left(\overline{e_{a}-e_{j}}\left(c_{M+N}^{-1}(x)\right) \overline{e_{M(j)}-e_{N(a)}}\left(c_{M+N}^{-1}(x)\right)\right) \\
& \prod_{\substack{j \in \mathrm{A}_{M}, a \in \mathrm{A}_{N} \\
j<a, M(j)>N(a)}}\left(\overline{e_{j}-e_{a}}\left(c_{M+N}^{-1}(x)\right) \overline{e_{N(a)}-e_{M(j)}}\left(c_{M+N}^{-1}(x)\right)\right) \\
& \left.\quad \prod_{\substack{j \in \mathrm{A}_{M}, a \in \mathrm{A}_{N} \\
j>a, M(j)>N(a)}}\left(\overline{e_{a}-e_{j}}\left(c_{M+N}^{-1}(x)\right) \overline{e_{N(a)}-e_{M(j)}}\left(c_{M+N}^{-1}(x)\right)\right)\right\} \\
& =(-1)^{\operatorname{Card}\left\{(j, a) \in \mathrm{A}_{M} \times \mathrm{A}_{N} \mid j<a, M(j)<N(a)\right\}+\operatorname{Card}\left\{(j, a) \in \mathrm{A}_{M} \times \mathrm{A}_{N} \mid j>a, M(j)>N(a)\right\}} \\
& =(-1)^{\prec \mathrm{A}_{M}, \mathrm{~A}_{N}} \succ+\prec \operatorname{im}(N), \operatorname{im}(M) \succ .
\end{aligned}
$$

Ce calcul permet aussi en prenant $M=N$ d'obtenir

$$
\begin{gathered}
\operatorname{sign}\left\{\prod_{\substack{j \in \mathrm{A}_{N}, a \in \mathrm{A}_{N} \\
j<a, N(j)<N(a)}}\left(\overline{e_{j}-e_{a}}\left(c_{M+N}^{-1}(x)\right) \overline{e_{N(j)}-e_{N(a)}}\left(c_{M+N}^{-1}(x)\right)\right)\right. \\
\left.\quad \prod_{\substack{j \in \mathrm{A}_{N}, a \in \mathrm{A}_{N} \\
j>a, N(j)<N(a)}}\left(\overline{e_{a}-e_{j}}\left(c_{M+N}^{-1}(x)\right) \overline{e_{N(j)}-e_{N(a)}}\left(c_{M+N}^{-1}(x)\right)\right)\right\} \\
=(-1)^{\operatorname{Card}\left\{(j, a) \in \mathrm{A}_{N} \times \mathrm{A}_{N} \mid j<a, N(j)<N(a)\right\}} \\
=(-1)^{\operatorname{Card}\left(\mathrm{A}_{N}\right)\left(\operatorname{Card}\left(\mathrm{A}_{N}\right)-1\right) / 2} \varepsilon(N) .
\end{gathered}
$$

Puis, pour $j \in I_{M+N} \cup J_{M+N}{ }^{(2)}$ et $a \in \mathrm{A}_{N}$, on a

$$
\begin{aligned}
\overline{e_{j}-e_{a}}\left(c_{M+N}^{-1}(x)\right) & =-\mathrm{i} x_{j}+u_{a}+\mathrm{i} v_{a} \\
\overline{e_{j}-e_{N(a)}}\left(c_{M+N}^{-1}(x)\right) & =-\mathrm{i} x_{j}-u_{a}+\mathrm{i} v_{a}
\end{aligned}
$$

ainsi

$$
\overline{e_{j}-e_{a}}\left(c_{M+N}^{-1}(x)\right) \overline{e_{j}-e_{N(a)}}\left(c_{M+N}^{-1}(x)\right)<0
$$

$\overline{{\text { (2) } I_{M+N} \cup J_{M+N}}}=\left(I_{M} \cup J_{M}\right) \cap\left(I_{N} \cup J_{N}\right)$ 
et

$$
\begin{gathered}
\operatorname{sign}\left\{\prod_{\substack{j \in I_{M+N}, a \in A_{N} \\
j<a}}\left(\overline{e_{j}-e_{a}}\left(c_{M+N}^{-1}(x)\right) \overline{e_{j}-e_{N(a)}}\left(c_{M+N}^{-1}(x)\right)\right)\right. \\
\prod_{\substack{j \in I_{M+N}, a \in A_{N} \\
j>a}}\left(\overline{e_{a}-e_{j}}\left(c_{M+N}^{-1}(x)\right) \overline{e_{j}-e_{N(a)}}\left(c_{M+N}^{-1}(x)\right)\right) \\
\quad \prod_{\substack{j \in J_{M+N}, a \in \mathrm{A}_{N} \\
j<N(a)}}\left(\overline{e_{a}-e_{j}}\left(c_{M+N}^{-1}(x)\right) \overline{e_{j}-e_{N(a)}}\left(c_{M+N}^{-1}(x)\right)\right) \\
\left.\quad \prod_{\substack{j \in J_{M+N}, a \in \mathrm{A}_{N} \\
j>N(a)}}\left(\overline{e_{a}-e_{j}}\left(c_{M+N}^{-1}(x)\right) \overline{e_{N(a)}-e_{j}}\left(c_{M+N}^{-1}(x)\right)\right)\right\} \\
=(-1)^{\operatorname{Card}\left\{(j, a) \in I_{M+N} \times \mathrm{A}_{N} \mid j<a\right\}+\operatorname{Card}\left\{(j, a) \in J_{M+N} \times \mathrm{A}_{N} \mid j>N(a)\right\}} \\
=(-1)^{\prec I_{M+N}, \mathbf{A}_{N} \succ+\prec \operatorname{im}(N), J_{M+N} \succ}
\end{gathered}
$$

On a l'égalité

$$
\begin{aligned}
\prec \mathrm{A}_{M}, \mathrm{~A}_{N} \succ+ & \prec \operatorname{im}(N), \operatorname{im}(M) \succ+\prec I_{M+N}, \mathrm{~A}_{N} \succ+\prec \operatorname{im}(N), J_{M+N} \succ \\
=\prec \mathrm{A}_{M}, \mathrm{~A}_{N} \succ & +\prec \operatorname{im}(N), \operatorname{im}(M) \succ+\prec I, \mathrm{~A}_{N} \succ+\prec \mathrm{A}_{M}, \mathrm{~A}_{N} \succ \\
& +\prec \mathrm{A}_{N}, \mathrm{~A}_{N} \succ \\
+ & \prec \operatorname{im}(N), J \succ+\prec \operatorname{im}(N), \operatorname{im}(M) \succ \\
& +\prec \operatorname{im}(N), \operatorname{im}(N) \succ+\prec I, \mathrm{~A}_{N} \succ+\prec \operatorname{im}(N), J \succ
\end{aligned}
$$

Ainsi le produit des expressions $(5.1 .23),(5.1 .24),(5.1 .25)$ et (5.1.26) est égal à

$$
\varepsilon(N)(-1)^{\operatorname{Card}\left(\mathbf{A}_{N}\right)\left(\operatorname{Card}\left(\mathbf{A}_{N}\right)-1\right) / 2}(-1)^{\prec I, \mathbf{A}_{N}} \succ+\prec \operatorname{im}(N), J \succ \mathbf{e}_{N}(x)
$$

et cette expression représente

$$
\operatorname{sign}\left(\prod_{\alpha \in E} \bar{\alpha}\left(x_{1}+x_{2}\right)\right) .
$$

On en déduit le résultat en considérant (5.1.22).

Le lemme 5.1.31 est équivalent à :

Lemme 5.1.33. - Pour $M, N \in \Omega$ disjoints et $x \in \mathfrak{h}_{N+M}^{\text {reg }}$, on a

$$
\overline{\pi_{\mathfrak{h}_{N+M}}}\left(c_{N+M}^{-1}(x)\right)=\varepsilon(N) \mu(N) \overline{\pi_{\mathfrak{h}_{N}, M}}\left(c_{M}^{-1}\left(x_{1}\right)\right) D_{X_{N}}\left(x_{2}\right) \mid \operatorname{det}\left(\left.\operatorname{ad}(x)\right|_{\mathfrak{n}_{N}} \mid\right.
$$

où $x=x_{1}+x_{2}$ avec $x_{1} \in \mathfrak{h}_{U_{N}, M}$ et $x_{2} \in \mathfrak{h}_{X_{N}}$.

On rappelle que

$$
C_{\mathfrak{g}, \mathfrak{g}^{\prime}}^{\prime}=\frac{(-1)^{\mathrm{n}(\mathrm{n}-1) / 2} C_{\mathfrak{g}, \mathfrak{g}^{\prime}}}{\mathrm{p} ! \mathrm{q} !}
$$


Lemme 5.1.34. - On a l'égalité

$$
\frac{C_{\mathfrak{g}, \mathfrak{g}^{\prime}}^{\prime}}{C_{\mathfrak{g}\left(V_{a, b}\right), \mathfrak{g}^{\prime}\left(V_{i_{0}, j_{0}}^{\prime}\right)}^{\prime}}=\frac{16^{\mathrm{n}-1}}{\mathrm{pq}} .
$$

Démonstration. - Par définition, on a

$$
C_{\mathfrak{g}\left(V_{a, b}\right), \mathfrak{g}^{\prime}\left(V_{i_{0}, j_{0}}^{\prime}\right)}=2^{(\mathrm{n}-2)^{2}} \operatorname{sign}\left(V_{a, b}\right)^{\mathrm{n}-2} \operatorname{sign}\left(V_{i_{0}, j_{0}}^{\prime}\right)^{\mathrm{n}-2} \mathbf{i}^{(\mathrm{n}-2)^{2}} .
$$

ainsi,

et

$$
C_{\mathfrak{g}\left(V_{a, b}\right), \mathfrak{g}^{\prime}\left(V_{i_{0}, j_{0}}^{\prime}\right)}=\frac{2^{\mathrm{n}^{2}}}{16^{\mathrm{n}-1}} \operatorname{sign}\left(V^{\prime}\right)^{\mathrm{n}} \operatorname{sign}(V)^{\mathrm{n}} \mathrm{i}^{\mathrm{n}^{2}+4-4 \mathrm{n}}=\frac{C_{\mathfrak{g}, \mathfrak{g}^{\prime}}}{16^{\mathrm{n}-1}},
$$

$$
\frac{C_{\mathfrak{g}, \mathfrak{g}^{\prime}}^{\prime}}{C_{\mathfrak{g}\left(V_{a, b}\right), \mathfrak{g}^{\prime}\left(V_{i_{0}, j_{0}}^{\prime}\right)}^{\prime}}=\frac{16^{\mathrm{n}-1}}{\mathrm{pq}} .
$$

Démonstration du théorème 5.1.30. - On rappelle que l'on a la relation pour $x^{\prime} \in$ $\mathfrak{h}_{\varnothing}^{\prime \text { reg }}$ et $\phi \in \mathcal{D}(\mathfrak{g})$

$$
\operatorname{Chc}(\phi)_{\varnothing}\left(x^{\prime}\right)=C_{\mathfrak{g}, \mathfrak{g}^{\prime}}^{\prime} \sum_{M \in \Omega} \mathcal{R}_{M}\left(\phi_{M}\right) . \quad \text { cf. égalité (5.1.15), p. } 110
$$

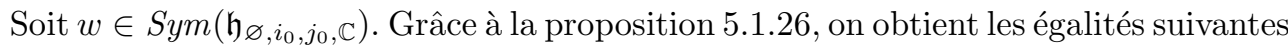

$$
\begin{aligned}
& \left\langle\partial(w) \mathbf{C h c}(\phi)_{\varnothing}\left(x^{\prime}\right)\right\rangle_{i_{0}, j_{0}}=C_{\mathfrak{g}, \mathfrak{g}^{\prime}}^{\prime} \sum_{M \in \Omega}\left\langle\partial(w) \mathcal{R}_{M}\left(\phi_{M}\right)\right\rangle_{i_{0}, j_{0}} \\
& =C_{\mathfrak{g}, \mathfrak{g}^{\prime}}^{\prime} \sum_{M \in \Omega}\left(\sum_{a \in \mathrm{A}_{M}}(-1)^{i_{0}+j_{0}+a+M(a)} \mathcal{R}_{M_{1}}^{V_{a, M(a)}, V_{i_{0}, j_{0}}^{\prime}}\left(\left\langle\partial(w) \widetilde{\mathcal{B}}_{a, M(a)}^{i_{0}, j_{0}}\left(\phi_{M}\right)\right\rangle_{i_{0}, j_{0}}\right)\right. \\
& \left.+\sum_{(a, b) \in I_{M} \times J_{M}}(-1)^{i_{0}+j_{0}+a+b} \mathcal{R}_{M}^{V_{a, b}, V_{i_{0}, j_{0}}^{\prime}}\left(\left\langle\partial(w) \widetilde{\mathcal{A}}_{a, b}^{i_{0}, j_{0},-\operatorname{sign}\left(V_{i_{0}}^{\prime}\right), \operatorname{sign}\left(V_{j_{0}}^{\prime}\right)}\left(\phi_{M}\right)\right\rangle_{i_{0}, j_{0}}\right)\right) .
\end{aligned}
$$

On rappelle que $M_{1}$ représente la restriction de $M$ à $\mathrm{A}_{M} \backslash\{a\}$. L'expression précédente s'écrit ainsi

$$
\begin{aligned}
& C_{\mathfrak{g}, \mathfrak{g}^{\prime}}^{\prime} \sum_{\substack{M \in \Omega \\
(a, b) \in I_{M} \times J_{M}}}(-1)^{i_{0}+j_{0}+a+b} \mathcal{R}_{M}^{V_{a, b}, V_{i_{0}, j_{0}}^{\prime}}\left(\left\langle\partial(w) \widetilde{\mathcal{B}}_{a, b}^{i_{0}, j_{0}}\left(\phi_{M+[a, b]}\right)\right\rangle_{i_{0}, j_{0}}\right) \\
+ & \sum_{\substack{M \in \Omega \\
(a, b) \in I_{M} \times J_{M}}}(-1)^{i_{0}+j_{0}+a+b} \mathcal{R}_{M}^{V_{a, b}, V_{i_{0}, j_{0}}^{\prime}}\left(\left\langle\partial(w) \widetilde{\mathcal{A}}_{a, b}^{i_{0}, j_{0},-\operatorname{sign}\left(V_{i_{0}}^{\prime}\right), \operatorname{sign}\left(V_{j_{0}}^{\prime}\right)}\left(\phi_{M}\right)\right\rangle_{i_{0}, j_{0}}\right) \\
= & \sum_{(a, b) \in I \times J}(-1)^{i_{0}+j_{0}+a+b} \sum_{M \in \Omega(I \backslash\{a\}, J \backslash\{b\})}
\end{aligned}
$$

$$
\mathcal{R}_{M}^{V_{a, b}, V_{i_{0}, j_{0}}^{\prime}}\left(\left\langle\partial(w) \widetilde{\mathcal{B}}_{a, b}^{i_{0}, j_{0}}\left(\phi_{M+[a, b]}\right)+\partial(w) \widetilde{\mathcal{A}}_{a, b}^{i_{0}, j_{0},-\operatorname{sign}\left(V_{i_{0}}^{\prime}\right), \operatorname{sign}\left(V_{j_{0}}^{\prime}\right)}\left(\phi_{M}\right)\right\rangle_{i_{0}, j_{0}}\right)
$$


Si $\alpha$ est une racine compacte, on a $\operatorname{sign}\left(V_{i_{0}}^{\prime}\right)=\operatorname{sign}\left(V_{j_{0}}^{\prime}\right)$ et on sait d'après la proposition 5.1.11 que

$$
\left\langle\partial(w) \widetilde{\mathcal{B}}_{a, b}^{i_{0}, j_{0}}\left(\phi_{M+[a, b]}\right)+\partial(w) \widetilde{\mathcal{A}}_{a, b}^{i_{0}, j_{0},-\operatorname{sign}\left(V_{i_{0}}^{\prime}\right), \operatorname{sign}\left(V_{j_{0}}^{\prime}\right)}\left(\phi_{M}\right)\right\rangle_{i_{0}, j_{0}}=0
$$

ainsi on a le point (1).

Notation. - Dans la fin de cette démonstration, on utilise par exemple la notation $v_{i_{0}}$ (resp. 0$)$ ) pour signifier que l'on évalue en $v_{a}$ par $v_{i_{0}}$ (resp. en $u_{a}$ par 0 ). La notation $\ldots, 0, v_{u_{a}}, \ldots$ signifie que l'on effectue les opérations définies ci-dessus sur $u_{a}$ et $v_{a}$ sans intervenir sur les autres variables.

Si $\alpha$ est une racine non compacte, on $\operatorname{argn}\left(V_{i_{0}}^{\prime}\right)=-\operatorname{sign}\left(V_{j_{0}}^{\prime}\right)$ et on sait par la même proposition que

$$
\begin{aligned}
\left\langle\partial(w) \widetilde{\mathcal{B}}_{a, b}^{i_{0}, j_{0}}\left(\phi_{M+[a, b]}\right)+\right. & \left.\partial(w) \widetilde{\mathcal{A}}_{a, b}^{i_{0}, j_{0},-\operatorname{sign}\left(V_{i_{0}}^{\prime}\right), \operatorname{sign}\left(V_{j_{0}}^{\prime}\right)}\left(\phi_{M}\right)\right\rangle_{i_{0}, j_{0}} \\
& =2\left|\operatorname{sign}\left(V_{j_{0}}^{\prime}\right)-\operatorname{sign}\left(V_{i_{0}}^{\prime}\right)\right| \pi^{2}\left\langle\partial\left(\operatorname{tr}_{i_{0}, j_{0}}^{a, b}(w)\right) \phi_{M}\right\rangle_{a, b}\left(v_{i_{0}}\right) \\
v_{a} & \\
& =4 \pi^{2} \mathrm{i} \partial\left(c_{a, b} t r_{i_{0}, j_{0}}^{a, b}(w)\right) \phi_{M+[a, b]}\left(\underset{u_{a}}{0, v_{i_{0}}}\right) .
\end{aligned}
$$

D'après le lemme 5.1.32, l'expression précédente s'écrit

$$
4(-1)^{a+b+n} \mathbf{i} \pi^{2}\left(\partial\left(c_{a, b} t r_{i_{0}, j_{0}}^{a, b}(w)\right)\left(\phi_{\mathfrak{n}_{[a, b]}}^{K}\right) G L\left(X_{[a, b]}\right)\right)_{M}^{V_{a, b}}\left(\underset{u_{a}}{0, v_{i_{0}}}\right) .
$$

L'expression (5.1.27) devient

$$
\begin{aligned}
& 4 \mathbf{i} \pi^{2}(-1)^{i_{0}+j_{0}+n} C_{\mathfrak{g}, \mathfrak{g}^{\prime}}^{\prime} \\
& \sum_{\substack{(a, b) \in I \times J \\
M \in \Omega(I \backslash\{a\}, J \backslash\{b\})}} \mathcal{R}_{M}^{V_{a, b}, V_{i_{0}, j_{0}}^{\prime}}\left(\partial\left(c_{a, b} t r_{i_{0}, j_{0}}^{a, b}(w)\right)\left(\phi_{\mathfrak{n}_{[a, b]}}^{K}\right) G L\left(X_{[a, b]}^{\prime}\right)\left(\underset{u_{a}}{0, v_{v_{0}}}\right)\right)_{M}^{V_{a, b}} \\
& =4 \mathrm{i} \pi^{2}(-1)^{i_{0}+j_{0}+n} \sum_{(a, b) \in I \times J} \frac{C_{\mathfrak{g}, \mathfrak{g}^{\prime}}^{\prime}}{C_{\mathfrak{g}\left(V_{a, b}\right), \mathfrak{g}^{\prime}\left(V_{i_{0}, j_{0}}^{\prime}\right)}^{\prime}} \\
& \operatorname{Chc}^{V_{a, b}, V_{i_{0}, j_{0}}^{\prime}}\left(\partial\left(c_{a, b} t r_{i_{0}, j_{0}}^{a, b}(w)\right)\left(\phi_{\mathfrak{n}_{[a, b]}}^{K}\right) G L\left(X_{[a, b]}\right)\left(\underset{u_{a} v_{a}}{0, v_{i_{0}}}\right)\right)_{\varnothing}
\end{aligned}
$$

où $x_{1}^{\prime}$ est la composante de $x^{\prime}$ dans $\mathfrak{h}_{V_{i_{0}, j_{0}}^{\prime}, \varnothing}^{\prime}$. D'après le lemme 5.1.34, on a

$$
C_{\mathfrak{g}, \mathfrak{g}^{\prime}}^{\prime} C^{\prime-1} \mathfrak{g}\left(V_{a, b}\right), \mathfrak{g}^{\prime}\left(V_{i_{0}, j_{0}}^{\prime}\right)=\frac{16^{\mathrm{n}-1}}{\mathrm{pq}}
$$

De plus, on remarque que

$$
\text { Chc }^{V_{a, b}, V_{i_{0}, j_{0}}^{\prime}}\left(\partial\left(c_{a, b} t r_{i_{0}, j_{0}}^{a, b}(w)\right)\left(\phi_{\mathfrak{n}_{[a, b]}}^{K}\right)^{G L\left(X_{[a, b]}\right)}\left(\ldots, \underset{u_{a}}{\left.0, v_{i_{0}}, \ldots\right)}\right)_{\varnothing}\left(x_{1}^{\prime}\right)\right.
$$


est indépendant du couple $(a, b) \in I \times J$. En effet, si on considère $\left(a^{\prime}, b^{\prime}\right) \in I \times J$, on note l'isométrie $u$ de $V$ suivante

$$
\left.u\right|_{V_{a, b} \cap V_{a^{\prime}, b^{\prime}}}=i d, u\left(x_{a}\right)=x_{a}^{\prime}, u\left(x_{a}^{\prime}\right)=x_{a}, u\left(x_{b}\right)=x_{b}^{\prime} \text { et } u\left(x_{b}^{\prime}\right)=x_{b} .
$$

On remarque alors que $u: V_{a, b} \rightarrow V_{a^{\prime}, b^{\prime}}$ est une isométrie, $u$ induit un automorphisme de $G$ et $\mathfrak{g}$ que l'on note $u$ qui vérifie $u(K)=K, u\left(\mathfrak{p}_{a, b}\right)=\mathfrak{p}_{a^{\prime}, b^{\prime}}$ et $u\left(\mathfrak{n}_{[a, b]}\right)=\mathfrak{n}_{\left[a^{\prime}, b^{\prime}\right]}$. De plus, $u$ induit un isomorphisme de $G\left(V_{a, b}\right)$ sur $G\left(V_{a^{\prime}, b^{\prime}}\right)$. On obtient pour $x \in$ $\mathfrak{g}\left(V_{a, b}\right) \oplus \mathfrak{g l}\left(X_{[a, b]}\right)$

$$
\phi_{\mathfrak{n}_{[a, b]}}^{K}(x)=\phi_{\mathfrak{n}_{\left[a^{\prime}, b^{\prime}\right]}^{K}}^{K}(u(x)) .
$$

Puis, pour $x \in \mathfrak{h}_{V_{a, b}^{\perp},[a, b]}^{\text {reg }}$

$$
\left(\phi_{\mathfrak{n}_{[a, b]}}^{K}\right)^{G L\left(X_{[a, b]}\right)}(x)=\left(\phi_{\mathfrak{n}_{\left[a^{\prime}, b^{\prime}\right]}^{K}}^{K}\right)^{G L\left(X_{\left[a^{\prime}, b^{\prime}\right]}\right)}(u(x))
$$

D'où

$$
\begin{aligned}
& \partial\left(c_{a, b} t r_{i_{0}, j_{0}}^{a, b}(w)\right)\left(\phi_{\mathfrak{n}_{[a, b]}}^{K}\right)^{G L\left(X_{[a, b]}\right)}\left(\ldots, \underset{u_{a}}{0}, v_{v_{a}}, \ldots\right) \\
& =\partial\left(c_{a^{\prime}, b^{\prime}} t r_{i_{0}, j_{0}}^{a^{\prime}, b^{\prime}}(w)\right)\left(\phi_{\mathfrak{n}_{\left[a^{\prime}, b^{\prime}\right]}^{K}}^{K}\right)^{G L\left(X_{\left[a^{\prime}, b^{\prime}\right]}\right)}\left(\ldots, \underset{u_{a}^{\prime}}{0, v_{i_{a}}^{\prime}}, \ldots\right) \circ u .
\end{aligned}
$$

On en déduit l'indépendance par rapport au couple $(a, b) \in I \times J$. En posant $(a, b)=$ $(p, n)$, l'expression (5.1.28) devient

$$
\begin{aligned}
& \mathbf{i} \pi^{2}(-1)^{i_{0}+j_{0}+n} 4^{2 \mathrm{n}-1} \\
& \mathbf{C h c} \mathbf{c}^{V_{p, n}, V_{i_{0}, j_{0}}^{\prime}}\left(\partial\left(c_{p, n} t r_{i_{0}, j_{0}}^{p, n}(w)\right)\left(\phi_{\mathbf{n}_{[p, n]}^{K}}^{K}\right)^{G L\left(X_{[p, n]}^{\perp}\right)}\left(\underset{u_{\mathrm{p}}}{0}, v_{v_{\mathrm{p}}}\right)\right)_{\varnothing}\left(x_{1}^{\prime}\right) .
\end{aligned}
$$

On en déduit le résultat.

\subsection{Pour une sous-algèbre de Cartan quelconque}

On note $I^{\prime}=\{1, \ldots, \mathrm{r}\}, J^{\prime}=\{\mathrm{r}+1, \ldots, \mathrm{n}\}$ et on rappelle que $\Omega^{\prime}$ désigne l'ensemble des injections d'une partie de $I^{\prime}$ dans une partie de $J^{\prime}$.

Soient $N \in \Omega^{\prime}, \mathcal{S}_{N}$ le système de racines fortement orthogonales associé et $\mathfrak{h}_{N}^{\prime}$ la sous-algèbre de Cartan de $\mathfrak{g}^{\prime}$ correspondante. Dans cette section, on étudie les propriétés de $\mathbf{C h c}(\phi)_{N}$ (définition en début de partie), on obtient les principaux résultats de ce travail, les théorèmes 5.2.4 et 5.2.5.

On considère les sous-espaces de $V^{\prime}$ suivants :

$$
V_{N, s}^{\prime}=\left\{w \in V^{\prime} \mid w_{i}^{\prime}=0 \text { si } i \notin \mathrm{A}_{N} \cup \operatorname{im}(N)\right\} \text { et } V_{N, c}^{\prime}=V_{N, s}^{\prime}{ }^{\perp} .
$$

On vérifie que $V_{N, c}^{\prime}=\left\{w \in V^{\prime} \mid a \cdot w=w \forall a \in A_{N}^{\prime}\right\}$ où $A_{N}^{\prime}$ est la partie déployée du sous-groupe de Cartan $H_{N}^{\prime}$ associé à $\mathfrak{h}_{N}^{\prime}$. Les sous-espaces $X_{N}^{\prime}$ et $Y_{N}^{\prime}$ de $V_{N, s}^{\prime}$ définis 
par

$$
X_{N}^{\prime}=\oplus_{i \in \mathrm{A}_{N}} \mathbb{C}\left(f_{i}^{\prime}-f_{N(i)}^{\prime}\right) \text { et } Y_{N}^{\prime}=\oplus_{i \in \mathrm{A}_{N}} \mathbb{C}\left(f_{i}^{\prime}+f_{N(i)}^{\prime}\right)
$$

sont des lagrangiens de $V_{N, s}^{\prime}, H_{N}^{\prime}$-invariants et complémentaires, c'est à dire qu'ils vérifient

$$
V_{N, s}^{\prime}=X_{N}^{\prime} \oplus Y_{N}^{\prime}
$$

La sous-algèbre de cartan $\mathfrak{h}_{N}^{\prime}$ se décompose ainsi :

$$
\mathfrak{h}_{N}^{\prime}=\mathfrak{a}_{N}^{\prime} \oplus \mathfrak{t}_{N}^{\prime}
$$

où $\mathfrak{a}_{N}^{\prime}\left(\right.$ resp. $\left.\mathfrak{t}_{N}^{\prime}\right)$ représente la partie déployée (resp. la partie compacte) de $\mathfrak{h}_{N}^{\prime}$.

Remarque. - On observe que $\operatorname{dim}_{\mathbb{C}} X_{N}^{\prime}=\operatorname{Card}\left(\mathbf{A}_{N}\right)=\operatorname{dim}\left(\mathfrak{a}_{N}^{\prime}\right)$.

On rappelle que $V$ est un espace de signature $(\mathrm{p}, \mathrm{q})$ avec $\mathrm{p} \leqslant \mathrm{q}$ ainsi index $(V)=\mathrm{p}$.

ThÉORÈme 5.2.1. - Si $\operatorname{index}(V)<\operatorname{dim}\left(\mathfrak{a}_{N}^{\prime}\right)$ alors pour $\phi \in \mathcal{D}(\mathfrak{g})$ et $x^{\prime} \in \mathfrak{h}_{N}^{\text {/reg }}$, on a

$$
\operatorname{Chc}(\phi)_{N}\left(x^{\prime}\right)=0 \text {. }
$$

Démonstration. - Cela découle directement de la remarque précédente et de la proposition 2.2.6.

On suppose maintenant que $\operatorname{dim}\left(\mathfrak{a}_{N}^{\prime}\right) \leqslant \operatorname{index}(V)$.

On sait que pour $N_{1}, N_{2} \in \Omega^{\prime}, \mathfrak{h}_{N_{1}}^{\prime}$ est conjuguée à $\mathfrak{h}_{N_{2}}^{\prime}$ si et seulement si $\operatorname{Card}\left(\mathrm{A}_{N_{1}}\right)=\operatorname{Card}\left(\mathrm{A}_{N_{2}}\right)$. Ainsi à conjugaison près, on peut supposer que $N$ vérifie $\mathrm{A}_{N} \subset I$ et $\operatorname{im}(N) \subset J$. L'injection $N$ est alors un élément de $\Omega, V_{s, N}^{\prime}$ est un sousespace de $V$ et $X_{N}^{\prime}, Y_{N}^{\prime}$ sont des lagrangiens de $V_{s, N}^{\prime}$ en tant que sous-espace de $V$. On pose $U_{N}=V_{s, N}^{\prime \perp} \subset V$. On remarque que $X_{N}^{\prime}=X_{N}, Y_{N}^{\prime}=Y_{N}$ et que cette définition de $U_{N}$ est cohérente avec la précédente définition (p.117). On note $\mathfrak{n}_{N}$ $\left(\right.$ resp. $\mathfrak{n}_{N}^{\prime}$ ) le radical unipotent de la sous-algèbre parabolique de $\mathfrak{g}$ (resp. $\mathfrak{g}^{\prime}$ ) préservant $X_{N}^{\prime}, n_{1}=\operatorname{dim}_{\mathbb{C}}\left(U_{N}\right)$ et $n_{1}^{\prime}=\operatorname{dim}_{\mathbb{C}}\left(V_{N, c}^{\prime}\right)$. Enfin, on pose

$$
D_{X_{N}}(x)=\left|\operatorname{det}\left(\left.\operatorname{ad}(x)\right|_{\mathfrak{g l}\left(X_{N}\right)}\right)\right|^{1 / 2}
$$

pour $x \in \mathfrak{a}_{N}$ et

$$
\psi^{G L\left(X_{N}\right)}(x)=D_{X_{N}}(x) \int_{G L\left(X_{N}\right) / H_{s, N}^{\prime}} \psi(g \cdot x) d \dot{g} .
$$

pour $\psi \in \mathcal{D}\left(\mathfrak{g l}\left(X_{N}\right)\right)$ et $x \in \mathfrak{a}_{N}^{\text {reg }}$ (les éléments réguliers de $\mathfrak{a}_{N}$ en tant que sous-algèbre de Cartan de $\mathfrak{g l}\left(X_{N}\right)$ ). L'égalité du théorème 2.2.7 s'écrit alors avec les notations introduites ci-dessus :

$$
\begin{aligned}
& \operatorname{Chc}(\phi)\left(x^{\prime}\right) \\
& \quad=\frac{\mathfrak{C}_{\mathfrak{g}, \mathfrak{g}^{\prime}, N}}{\mid \operatorname{det}\left(\left.\operatorname{ad}\left(x^{\prime}\right)\right|_{\left.\mathfrak{n}_{N}^{\prime}\right) \mid}\right.} \operatorname{Chc}^{U_{N}, V_{N, c}^{\prime}}\left(\int_{G L\left(X_{N}\right) / H_{s, N}^{\prime}} \phi_{\mathbf{n}_{N}}^{K}\left(g \cdot x_{s}^{\prime}+.\right) d \dot{g}\right)\left(x_{c}^{\prime}\right),
\end{aligned}
$$


où $H_{s, N}^{\prime}=H_{N}^{\prime}\left|X_{N}^{\prime}, x_{c}^{\prime}=x^{\prime}\right| V_{N, c}^{\prime}, x_{s}^{\prime}=x^{\prime} \mid X_{N}^{\prime}$. Le lemme suivant permet d'obtenir une nouvelle égalité.

Lemme 5.2.2. - Avec les notations introduites ci-dessus, on a

$\operatorname{Chc}(\phi)_{N}\left(x^{\prime}\right)=\mathfrak{C}_{\mathfrak{g}, \mathfrak{g}^{\prime}, N} \mu(N) \varepsilon(N) \mathbf{C h c}^{U_{N}, V_{N, c}^{\prime}}\left(\left(\phi_{\mathfrak{n}_{N}}^{K}\left(x_{s}^{\prime}+.\right)\right)^{G L\left(X_{N}\right)}\right)_{\varnothing}\left(x_{c}^{\prime}\right)$.

Démonstration. - D'après le lemme 5.1.33, on a pour $N, M$ disjoints et $x \in \mathfrak{h}_{N+M}^{\text {reg }}$

$$
\overline{\pi_{\mathfrak{h} \varnothing}}\left(c_{N+M}^{-1}(x)\right)=\mu(N) \varepsilon(N) \overline{\pi_{U_{N}, \varnothing}}\left(x_{1}\right) D_{X_{N}}\left(x_{2}\right)\left|\operatorname{det}\left(\operatorname{ad}(x)_{\mathfrak{n}_{N}}\right)\right|
$$

où $x=x_{1}+x_{2}$ avec $x_{1} \in \mathfrak{h}_{U_{N}, M}$ et $x_{2} \in \mathfrak{h}_{X_{N}}$. Ainsi, on obtient

$$
\begin{aligned}
\operatorname{Chc}(\phi)_{N}\left(x^{\prime}\right)=\mathfrak{C}_{\mathfrak{g}, \mathfrak{g}^{\prime}, N} \mu(N) \varepsilon(N) \mathbf{C h c}^{U_{N}, V_{N, c}^{\prime}} \\
\qquad\left(D_{X_{N}}\left(x_{s}^{\prime}\right) \int_{G L\left(X_{N}\right) / H_{s, N}^{\prime}} \phi_{\mathbf{n}_{N}}^{K}\left(g \cdot x_{s}^{\prime}+.\right) d \dot{g}\right)_{\varnothing}\left(x_{c}^{\prime}\right) .
\end{aligned}
$$

Pour $\mathfrak{h} \in \operatorname{Car}(\mathfrak{g})$, on note $\mathcal{W}_{c}(\mathfrak{h})$ l'espace topologique $\mathcal{H}_{c}\left(\mathfrak{h}^{\text {reg }}\right)$. On a le résultat suivant dû à Harish-Chandra :

Proposition 5.2.3 ([2, Lemme 3.2.2]). - Pour $M, N \in \Omega$, l'application

$$
\begin{aligned}
\mathcal{D}(\mathfrak{g}) & \longrightarrow \mathcal{W}_{c}\left(\mathfrak{h}_{M}\right) \\
\phi & \longmapsto \phi_{M}
\end{aligned}
$$

est continue. De même, l'application

$$
\begin{aligned}
\mathcal{D}\left(\mathfrak{g l}\left(X_{N}\right)\right) & \longrightarrow \mathcal{W}_{c}\left(\mathfrak{a}_{N}^{\prime}\right) \\
\theta & \longmapsto \theta^{G L\left(X_{N}\right)}
\end{aligned}
$$

est continue.

Remarque. - Comme la sous-algèbre de Cartan $\mathfrak{a}_{N}^{\prime} \in \operatorname{Car}\left(\mathfrak{g r}\left(X_{N}\right)\right)$ n'a pas de racines imaginaires, on a

$$
\theta^{G L\left(X_{N}\right)} \in \mathcal{D}\left(\mathfrak{a}_{N}^{\prime}\right) .
$$

Le but de cette sous-section est de montrer le résultat suivant :

ThÉORÈme 5.2.4. - Pour $\phi \in \mathcal{D}(\mathfrak{g})$, on a les propriétés suivantes :

(1) La fonction $\mathbf{C h c}(\phi)\left(x^{\prime}\right)$ est définie, lisse sur $\mathfrak{g}^{\text {/reg }}$ et $G^{\prime}$-invariante.

(2) Pour $N \in \Omega^{\prime}, w \in \operatorname{Sym}\left(\mathfrak{h}_{N, \mathbb{C}}^{\prime}\right)$ et $\mathcal{K}^{\prime}$ une partie compacte de $\mathfrak{h}_{N}^{\prime}$, on a

$$
\sup _{x^{\prime} \in \mathcal{K}^{\prime} \cap \mathfrak{h}_{N}^{\text {reg }}}\left|\partial(w) \operatorname{Chc}(\phi)_{N}\left(x^{\prime}\right)\right|<\infty .
$$

(3) Pour $N \in \Omega^{\prime}$, l'application

$$
\begin{aligned}
\mathcal{D}(\mathfrak{g}) & \longrightarrow \mathcal{W}\left(\mathfrak{h}_{N}^{\prime}\right) \\
\phi & \longmapsto \mathbf{C h c}(\phi)_{N}\left(x^{\prime}\right)
\end{aligned}
$$

est continue. 
Démonstration. - Montrons la $G^{\prime}$-invariance. Soient $\mathfrak{h}_{1}^{\prime}, \mathfrak{h}_{2}^{\prime} \in \operatorname{Car}\left(\mathfrak{g}^{\prime}\right)$ deux sousalgèbres de Cartan conjuguées, il existe alors $g \in G^{\prime}$ tel que $\mathfrak{h}_{1}^{\prime}=g \cdot \mathfrak{h}_{2}^{\prime}$. Avec les notations introduites dans la sous-section 2.1.1, on a $\mathfrak{a}_{1}^{\prime \prime}=g \cdot \mathfrak{a}_{2}^{\prime \prime}$ et $A_{1}^{\prime \prime \prime}=g \cdot A_{2}^{\prime \prime \prime}$. Pour une fonction $\psi$ définie sur $\mathfrak{a}_{1}^{\prime \prime}$, on pose $\psi^{g}$ la fonction définie sur $\mathfrak{a}_{2}^{\prime \prime}$ par $\psi^{g}(x)=$ $\psi(g \cdot x)$. Pour une distribution $T$ sur $\mathfrak{a}_{1}^{\prime \prime}$, on note $T^{g}$ la distribution définie sur $\mathfrak{a}_{2}^{\prime \prime}$ par $T^{g}(\psi)=T\left(\psi^{g^{-1}}\right)$. On obtient avec les notations de la proposition 1.1.6 :

$$
C h c_{\mathfrak{a}_{1}^{\prime \prime}}^{g}=C h c_{\mathfrak{a}_{2}^{\prime \prime}} .
$$

Pour $x^{\prime} \in \mathfrak{h}_{i}^{\text {reg }}$ avec $i \in\{1,2\}$, on note $I_{i, x^{\prime}}$ l'injection $x \in \mathfrak{g} \hookrightarrow x+x^{\prime} \in \mathfrak{a}_{i}^{\prime \prime}$. Par définition, la distribution $C h c_{x^{\prime}}$ est la restriction de $C h c$ par $I_{i, x^{\prime}}$ si $x^{\prime} \in \mathfrak{h}_{i}^{\text {reg }}$. Pour $\psi \in \mathcal{D}(\mathfrak{g})$ et $x^{\prime} \in \mathfrak{h}_{2}^{\text {reg }}$, on obtient les égalités suivantes

$$
\begin{aligned}
C h c_{x^{\prime}}(\psi) & =I_{2, x^{\prime}}^{*} C h c_{\mathfrak{a}_{2}^{\prime \prime}}(\psi) \\
& =I_{2, x^{\prime}}^{*} C h c_{\mathfrak{a}_{1}^{\prime \prime}}^{g}(\psi)=\left(g \cdot I_{2, x^{\prime}}\right)^{*} C h c_{\mathfrak{a}_{1}^{\prime \prime}}(\psi) \\
& =I_{1, g \cdot x^{\prime}}^{*} C h c_{\mathfrak{a}_{1}^{\prime \prime}}(\psi)=C h c_{g \cdot x^{\prime}}(\psi)
\end{aligned}
$$

Par définition, on a $\operatorname{Chc}(\psi)\left(x^{\prime}\right)=C h c_{x^{\prime}}(\psi)$, ainsi on en déduit la $G^{\prime}$-invariance de la fonction $\mathbf{C h c}(\psi)$.

On considère $w^{\prime}=w_{1}^{\prime} w_{2}^{\prime} \in \operatorname{Sym}\left(\mathfrak{h}_{N, \mathbb{C}}^{\prime}\right)$ avec $w_{1}^{\prime} \in \operatorname{Sym}\left(\mathfrak{a}_{N, \mathbb{C}}^{\prime}\right)$ et $w_{2}^{\prime} \in \operatorname{Sym}\left(\mathfrak{t}_{N, \mathbb{C}}^{\prime}\right)$ et une partie compacte $\mathcal{K}^{\prime}$ de $\mathfrak{h}_{N}^{\prime}$. Il existe alors des parties compactes $\mathcal{K}_{1}^{\prime}$ et $\mathcal{K}_{2}^{\prime}$ de $\mathfrak{a}_{N}^{\prime}$ et $\mathfrak{t}_{N}^{\prime}$ telles que $\mathcal{K}^{\prime} \subset \mathcal{K}_{1}^{\prime}+\mathcal{K}_{2}^{\prime}$. D'après le lemme 5.2 .2 , on a la relation

$$
\begin{aligned}
\partial(w) & \operatorname{Chc}(\psi)_{N}\left(x^{\prime}\right) \\
& =\mathfrak{C}_{\mathfrak{g}, \mathfrak{g}^{\prime}, N} \mu(N) \varepsilon(N) \partial\left(w_{2}\right) \mathbf{C h c}^{U_{N}, V_{N, c}^{\prime}}\left(\partial\left(w_{1}\right) \phi^{G L\left(X_{N}^{\prime}\right)}\left(x_{s}^{\prime}+.\right)\right)_{\varnothing}\left(x_{c}^{\prime}\right)
\end{aligned}
$$

où $\phi=\psi_{\mathfrak{n}_{N}}^{K} \in \mathcal{D}\left(\mathfrak{g l}\left(X_{N}^{\prime}\right) \oplus \mathfrak{g}\left(U_{N}\right)\right)$. Soit $\mathcal{K}$ une partie compacte de $\mathfrak{g}$. Il existe alors une partie compacte $\mathcal{K}_{1}$ de $\mathfrak{g l}\left(X_{N}^{\prime}\right) \oplus \mathfrak{g}\left(U_{N}\right)$ telle que l'application

$$
\begin{aligned}
\mathcal{D}_{\mathcal{K}}(\mathfrak{g}) & \longrightarrow \mathcal{D}_{\mathcal{K}_{1}}\left(\mathfrak{g l}\left(X_{N}^{\prime}\right) \oplus \mathfrak{g}\left(U_{N}\right)\right) \\
\psi & \longmapsto \psi_{\mathfrak{n}_{N}}^{K}
\end{aligned}
$$

soit définie et continue. On fixe ensuite deux compacts $\mathcal{K}_{2}$ et $\mathcal{K}_{3}$ respectivement de $\mathfrak{g l}\left(X_{N}^{\prime}\right)$ et $\mathfrak{g}\left(U_{N}\right)$ tels que $\mathcal{K}_{1} \subset \mathcal{K}_{2}+\mathcal{K}_{3}$. D'après la proposition 5.1.25, l'application

$$
\begin{aligned}
\mathcal{D}\left(\mathfrak{g}\left(U_{N}\right)\right) & \longrightarrow \mathcal{W}\left(\mathfrak{t}_{N}^{\prime}\right) \\
\theta & \longmapsto x_{c}^{\prime} \in \mathfrak{t}_{N}^{\text {'reg }} \mapsto \mathbf{C h c}^{U_{N}, V_{N, c}^{\prime}(\theta)_{\varnothing}\left(x_{c}^{\prime}\right)}
\end{aligned}
$$

est continue, où $\mathfrak{t}_{N}^{\text {reg }}$ désigne l'ensemble des éléments réguliers de $\mathfrak{t}_{N}^{\prime}$ en tant que sousalgèbre de Cartan de $\mathfrak{g}^{\prime}\left(V_{N, c}^{\prime}\right)$. Ainsi, il existe une famille d'éléments $t_{1}, \ldots, t_{m}$ de $\operatorname{Sym}\left(\mathfrak{g}\left(U_{N}\right)_{\mathbb{C}}\right)$ telle que

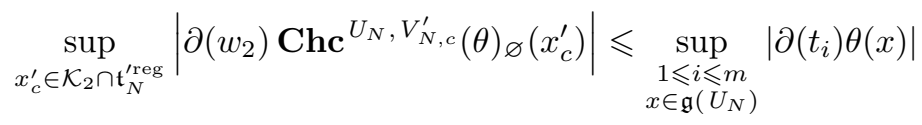


pout tout $\theta \in \mathcal{D}_{\mathcal{K}_{3}}\left(\mathfrak{g}\left(U_{N}\right)\right)$ Comme $\partial\left(w_{1}\right) \phi_{N}^{G L\left(X_{N}^{\prime}\right)}\left(x_{s}^{\prime}+.\right) \in \mathcal{D}_{\mathcal{K}_{3}}\left(\mathfrak{g}\left(U_{N}\right)\right)$, on obtient l'inégalité suivante

$$
\begin{gathered}
\sup _{x_{c}^{\prime} \in \mathcal{K}_{2} \cap \mathfrak{t}_{N}^{\prime \text { reg }}}\left|\partial\left(w_{2}\right) \mathbf{C h c}^{U_{N}, V_{N, c}^{\prime}}\left(\partial\left(w_{1}\right) \phi^{G L\left(X_{N}^{\prime}\right)}\left(x_{s}^{\prime}+.\right)\right)_{\varnothing}\left(x_{c}^{\prime}\right)\right| \\
\leqslant \sup _{\substack{1 \leqslant i \leqslant m \\
x \in \mathfrak{g}\left(U_{N}\right)}}\left|\partial\left(t_{i} w_{1}\right)\left(\phi^{G L\left(X_{N}^{\prime}\right)}\right)\left(x_{s}^{\prime}+x\right)\right|
\end{gathered}
$$

pour $x_{s}^{\prime} \in \mathfrak{a}_{N}^{\prime}$. On sait ensuite d'après la proposition 5.2.3 que l'application

$$
\begin{aligned}
\mathcal{D}_{\mathcal{K}_{2}}\left(\mathfrak{g l} l\left(X_{N}\right)\right) & \longrightarrow \mathcal{W}_{c}\left(\mathfrak{a}_{N}\right) \\
\theta & \longmapsto \theta^{G L\left(X_{N}\right)}
\end{aligned}
$$

est continue, ainsi il existe des éléments $y_{1}, \ldots, y_{q}$ de $\operatorname{Sym}\left(\mathfrak{g l}\left(X_{N}\right)_{\mathbb{C}}\right)$ tels que

$$
\sup _{x \in \mathfrak{a}_{N}^{\text {reg }}}\left|\partial\left(w_{1}\right) \theta^{G L\left(X_{N}\right)}(x)\right| \leqslant \sup _{\substack{1 \leqslant j \leqslant q \\ x \in \mathfrak{g l}\left(X_{N}\right)}}\left|\partial\left(y_{j}\right) \theta(x)\right|
$$

pour tout $\theta \in \mathcal{D}_{\mathcal{K}_{2}}\left(\mathfrak{g l}\left(X_{N}\right)\right)$. Cette dernière inégalité permet d'obtenir avec (5.2.4), la majoration

$$
\begin{aligned}
& \sup _{x^{\prime} \in \mathcal{K}^{\prime} \cap \mathfrak{h}_{N}^{\prime \text { reg }}}\left|\partial\left(w_{2}\right) \mathbf{C h c}^{U_{N}, V_{N, c}^{\prime}}\left(\partial\left(w_{1}\right) \phi^{G L\left(X_{N}\right)}\left(x_{s}^{\prime}+.\right)\right)_{\varnothing}\left(x_{c}^{\prime}\right)\right| \\
& \leqslant \sup _{x_{s}^{\prime} \in \mathfrak{a}_{N}^{\prime \text { reg }} \cap \mathcal{K}_{1}}\left(\sup _{\substack{1 \leqslant i \leqslant m \\
x \in \mathfrak{g}\left(U_{N}\right)}}\left|\partial\left(t_{i} w_{1}\right)\left(\phi^{G L\left(X_{N}\right)}\right)\left(x_{s}^{\prime}+x\right)\right|\right) \\
& \leqslant \sup _{\substack{1 \leqslant i \leqslant m \\
1 \leqslant j \leqslant q \\
x \in \mathfrak{g}\left(U_{N}\right) \oplus \mathfrak{g l}\left(X_{N}\right)}}\left|\partial\left(t_{i} y_{j}\right)(\phi)(x)\right|
\end{aligned}
$$

Cette inégalité montre le point (2). Il existe d'après (5.2.3) une famille d'éléments $z_{1}, \ldots, z_{l}$ de $\operatorname{Sym}\left(\mathfrak{g}_{\mathbb{C}}\right)$ telle que

$$
\sup _{\substack{1 \leqslant i \leqslant m \\ 1 \leqslant j \leqslant q \\ \in \mathfrak{g}\left(U_{N}\right)+\mathfrak{g}\left(V_{s}^{\prime}\right)}}\left|\partial\left(t_{i} y_{j}\right) \phi(x)\right| \leqslant \sup _{\substack{1 \leqslant k \leqslant l \\ x \in \mathfrak{g}}}\left|\partial\left(z_{k}\right) \psi(x)\right| .
$$

On obtient ainsi avec (5.2.5), l'inégalité

$$
\sup _{x^{\prime} \in \mathcal{K}^{\prime} \cap \mathfrak{h}_{N}^{\prime \text { reg }}}\left|\partial(w) \mathbf{C h c}(\psi)_{N}\left(x^{\prime}\right)\right| \leqslant \sup _{\substack{1 \leqslant k \leqslant l \\ x \in \mathfrak{g}}}\left|\partial\left(z_{k}\right) \psi(x)\right| .
$$

On en déduit le point (3). 
Le théorème suivant complète les propriétés locales.

ThÉORÈme 5.2.5. - Pour $\phi \in \mathcal{D}(\mathfrak{g})$, on a les propriétés suivantes

(1) Pour $N \in \Omega^{\prime}$ et $w \in \operatorname{Sym}\left(\mathfrak{h}_{N, \mathbb{C}}^{\prime}\right)$, la fonction

$$
\partial(w) \mathbf{C h c}(\phi)_{N}\left(x^{\prime}\right)
$$

se prolonge par continuité sur $\mathfrak{h}_{N}^{\text {,nc }}$.

(2) Pour $\alpha=e_{i_{0}}-e_{j_{0}} \in \Sigma^{i}\left(\mathfrak{h}_{N}^{\prime}\right)$ (racines imaginaires) non compacte et $w \in$ $\operatorname{Sym}\left(\mathfrak{h}_{N, \mathbb{C}}^{\prime}\right)$, on a

(a) $S i \operatorname{index}(V)>\operatorname{dim}\left(\mathfrak{a}_{N}^{\prime}\right)$,

$$
\left\langle\partial(w) \mathbf{C h c}(\phi)_{N}\right\rangle_{i_{0}, j_{0}}=\mathbf{i} \pi^{2} 4^{2 \mathbf{n}-4 m-1} \frac{\mathfrak{C}_{\mathfrak{g}, \mathfrak{g}^{\prime}, N}}{\mathfrak{C}_{\mathfrak{g}, \mathfrak{g}^{\prime}, N+\left[i_{0}, j_{0}\right]}} \partial\left(c_{i_{0}, j_{0}}(w)\right) \mathbf{C h c}(\phi)_{N+\left[i_{0}, j_{0}\right]}
$$

où $m=\operatorname{Card}\left(\mathrm{A}_{N}\right)$.

(b) $S i \operatorname{index}(V)=\operatorname{dim}\left(\mathfrak{a}_{N}^{\prime}\right)$,

$$
\left\langle\partial(w) \operatorname{Chc}(\phi)_{N}\right\rangle_{i_{0}, j_{0}}=0 .
$$

Démonstration. - On souhaite montrer le point (1). $\operatorname{Si} \operatorname{Card}\left(\mathrm{A}_{N}\right)>\operatorname{index}(V)$, le point (1) est vérifié. Sinon, on peut supposer comme $\mathbf{C h c}(\psi)$ est $G^{\prime}$-invariante que $N \in \Omega \cap \Omega^{\prime}$. L'égalité du lemme 5.2.2 permet d'obtenir $w \in \operatorname{Sym}\left(\mathfrak{h}_{N, \mathbb{C}}^{\prime}\right)$

$$
\begin{aligned}
\partial(w) \operatorname{Chc}(\psi)_{N}\left(x^{\prime}\right) & \\
\quad & =\mathfrak{C}_{\mathfrak{g}, \mathfrak{g}^{\prime}, N} \mu(N) \varepsilon(N) \partial\left(w_{2}\right) \mathbf{C h c}^{U_{N}, V_{N, c}^{\prime}}\left(\partial\left(w_{1}\right) \phi^{G L\left(X_{N}\right)}\left(x_{s}^{\prime}+.\right)\right)_{\varnothing}\left(x_{c}^{\prime}\right) .
\end{aligned}
$$

où $w_{1} \in \operatorname{Sym}\left(\mathfrak{a}_{N, \mathbb{C}}^{\prime}\right), w_{2} \in \operatorname{Sym}\left(\mathfrak{t}_{N, \mathbb{C}}^{\prime}\right)$ et $w=w_{1} w_{2}$. Comme $X_{N}^{\prime}=X_{N}$, on utilise $X_{N}$ uniquement. Pour $z \in \mathfrak{g}\left(U_{N}\right)$ et $w_{3} \in \operatorname{Sym}\left(\mathfrak{g}\left(U_{N}\right)_{\mathbb{C}}\right)$, la fonction

$$
x_{s}^{\prime} \in \mathfrak{a}_{N}^{\text {'reg }} \mapsto \partial\left(w_{1}\right) \partial\left(w_{3}\right) \phi^{G L\left(X_{N}\right)}\left(x_{s}^{\prime}+z\right)
$$

se prolonge par continuité sur $\mathfrak{a}_{N}^{\prime}$. Pour $x_{s}^{\prime}, y_{s}^{\prime} \in \mathcal{K}_{4}$ où $\mathcal{K}_{4}$ est une partie convexe compacte de $\mathfrak{a}_{N}^{\prime}$, il existe une famille d'éléments $v_{1}, \ldots, v_{m}$ de $\operatorname{Sym}\left(\mathfrak{a}_{N, \mathbb{C}}^{\prime}\right)$ telle que

$$
\begin{aligned}
& \left|\partial\left(w_{1} w_{3}\right) \phi^{G L\left(X_{N}\right)}\left(x_{s}^{\prime}+z\right)-\partial\left(w_{1} w_{3}\right) \phi^{G L\left(X_{N}\right)}\left(y_{s}^{\prime}+z\right)\right| \\
& \leqslant\left\|x_{s}^{\prime}-y_{s}^{\prime}\right\| \sup _{\substack{x^{\prime} \in \mathcal{K}_{4} \\
1 \leqslant i \leqslant m}}\left|\partial\left(v_{i} w_{1} w_{3}\right) \phi^{G L\left(X_{N}\right)}\left(x^{\prime}+z\right)\right|
\end{aligned}
$$

pour une norme donnée sur $\mathfrak{a}_{N}^{\prime}$ en utilisant l'inégalité des accroissements finis. On sait qu'il existe une famille d'éléments $z_{1}, \ldots, z_{r}$ de $\operatorname{Sym}\left(\mathfrak{g r}\left(X_{N}\right)_{\mathbb{C}}\right)$ telle que

$$
\sup _{\substack{x^{\prime} \in \mathcal{K}_{4} \\ 1 \leqslant i \leqslant m}}\left|\partial\left(v_{i} w_{1} w\right) \phi^{G L\left(X_{N}\right)}\left(x^{\prime}+z\right)\right| \leqslant \sup _{\substack{1 \leqslant j \leqslant r \\ x \in \mathfrak{g l}\left(X_{N}\right)}}\left|\partial\left(z_{j} w\right) \phi(x+z)\right| .
$$


On en déduit alors l'inégalité suivante

$$
\begin{aligned}
\sup _{z \in \mathfrak{g}\left(U_{N}\right)} \mid \partial(w)\left(\partial\left(w_{1}\right) \phi^{G L\left(X_{N}\right)}\left(x_{s}^{\prime}+z\right)\right. & \left.-\partial\left(w_{1}\right) \phi^{G L\left(X_{N}\right)}\left(y_{s}^{\prime}+z\right)\right) \mid \\
& \leqslant\left\|x_{s}^{\prime}-y_{s}^{\prime}\right\| \sup _{\substack{1 \leqslant j \leqslant r \\
y \in \mathfrak{g}\left(U_{N}\right)+\mathfrak{g}\left(X_{N}\right)}}\left|\partial\left(z_{j} w\right) \phi(y)\right| .
\end{aligned}
$$

On a ainsi montré que l'application

$$
\begin{aligned}
\mathfrak{a}_{N}^{\prime} & \longrightarrow \mathcal{D}_{\mathcal{K}_{3}}\left(\mathfrak{g}\left(U_{N}\right)\right) \\
x_{s}^{\prime} & \longmapsto \partial\left(w_{1}\right) \phi^{G L\left(X_{N}\right)}\left(x_{s}^{\prime}+.\right)
\end{aligned}
$$

est continue. On en déduit en utilisant le point (3) du théorème 5.2.4 que l'application

$$
\begin{aligned}
\mathfrak{a}_{N}^{\prime} & \longrightarrow \mathcal{W}\left(\mathfrak{t}_{N}^{\prime}\right) \\
x_{s}^{\prime} & \longmapsto \mathbf{C h c}^{U_{N}, V_{N, c}^{\prime}}\left(\partial\left(w_{1}\right) \phi^{G L\left(X_{N}\right)}\left(x_{s}^{\prime}+.\right)\right)_{\varnothing}
\end{aligned}
$$

est continue. On note $\mathfrak{t}_{N}^{\prime n c}$ les éléments de $\mathfrak{t}_{N}$ qui ne s'annulent pour aucune racine imaginaire de $\mathfrak{t}_{N}$. On remarque que les racines imaginaires de $\mathfrak{t}_{N}$ sont les restrictions des racines imaginaires de $\mathfrak{h}_{N}$. Soient $P$ l'adhérence d'une composante connexe de $\mathfrak{t}_{N}^{\prime \text { nc }}$, $Q$ une partie compacte convexe de $P$ et $R$ une partie compacte de $\mathfrak{a}_{N}^{\prime}$. On considère $x^{\prime}, y^{\prime} \in \mathfrak{h}_{N}^{\prime}$ tels que $x_{c}^{\prime}, y_{c}^{\prime} \in Q$ et $x_{s}^{\prime}, y_{s}^{\prime} \in R$. On sait d'après la proposition 5.1.25 que l'application

$$
x_{c}^{\prime} \in P \longmapsto \partial\left(w_{2}\right) \mathbf{C h c}^{U_{N}, V_{N, c}^{\prime}}\left(\partial\left(w_{1}\right) \phi^{G L\left(X_{N}\right)}\left(x_{s}^{\prime}+.\right)\right)_{\varnothing}\left(x_{c}^{\prime}\right)
$$

est continue pour tout $w_{2} \in \operatorname{Sym}\left(\mathfrak{t}_{N, \mathbb{C}}^{\prime}\right)$. Il existe donc une famille d'éléments $t_{1}, \ldots, t_{m} \in \operatorname{Sym}\left(\mathfrak{t}_{N, \mathbb{C}}\right)$ tels que

$$
\begin{aligned}
& \mid \partial\left(w_{2}\right) \mathbf{C h c}^{U_{N}, V_{N, c}^{\prime}}\left(\partial\left(w_{1}\right) \phi^{G L\left(X_{N}\right)}\left(x_{s}^{\prime}+.\right)\right)_{\varnothing}\left(x_{c}^{\prime}\right) \\
& -\partial\left(w_{2}\right) \mathbf{C h c}^{U_{N}, V_{N, c}^{\prime}}\left(\partial\left(w_{1}\right) \phi^{G L\left(X_{N}\right)}\left(x_{s}^{\prime}+.\right)\right)_{\varnothing}\left(y_{c}^{\prime}\right) \mid \\
& \leqslant\left\|x_{c}^{\prime}-y_{c}^{\prime}\right\| \sup _{\substack{1 \leqslant i \leqslant m \\
z_{c}^{\prime} \in Q}}\left|\partial\left(t_{i}\right) \mathbf{C h c}^{U_{N}, V_{N, c}^{\prime}}\left(\partial\left(w_{1}\right) \phi^{G L\left(X_{N}\right)}\left(x_{s}^{\prime}+.\right)\right)_{\varnothing}\left(z_{c}^{\prime}\right)\right|
\end{aligned}
$$

D'après (5.2.7), il existe une constante $M>0$ telle que

$$
\sup _{\substack{1 \leqslant i \leqslant m \\ z_{c}^{\prime} \in Q \\ x_{s}^{\prime} \in R}}\left|\partial\left(t_{i}\right) \mathbf{C h c}^{U_{N}, V_{N, c}^{\prime}}\left(\partial\left(w_{1}\right) \phi^{G L\left(X_{N}\right)}\left(x_{s}^{\prime}+.\right)\right)_{\varnothing}\left(z_{c}^{\prime}\right)\right| \leqslant M
$$

ainsi, on a

$$
\begin{aligned}
\mid \partial\left(w_{2}\right) \mathbf{C h c}^{U_{N}, V_{N, c}^{\prime}}\left(\partial\left(w_{1}\right) \phi^{G L\left(X_{N}\right)}\left(x_{s}^{\prime}+.\right)\right)_{\varnothing}\left(x_{c}^{\prime}\right) & \\
& -\partial\left(w_{2}\right) \mathbf{C h c}^{U_{N}, V_{N, c}^{\prime}}\left(\partial\left(w_{1}\right) \phi^{G L\left(X_{N}\right)}\left(x_{s}^{\prime}+.\right)\right)_{\varnothing}\left(y_{c}^{\prime}\right) \mid \leqslant\left\|x_{c}^{\prime}-y_{c}^{\prime}\right\| M .
\end{aligned}
$$


Cette inégalité permet avec (5.2.7) d'obtenir que l'application

$$
\begin{aligned}
Q \times R & \longrightarrow \mathbb{C} \\
\left(x_{c}^{\prime}, x_{s}^{\prime}\right) & \longmapsto \partial\left(w_{2}\right) \mathbf{C h c}^{U_{N}, V_{N, c}^{\prime}}\left(\partial\left(w_{1}\right) \phi^{G L\left(X_{N}\right)}\left(x_{s}^{\prime}+.\right)\right)_{\varnothing}\left(x_{c}^{\prime}\right)
\end{aligned}
$$

est continue. On en déduit que $\partial(w) \mathbf{C h c}(\psi)_{N}$ est continue sur l'ensemble $\mathfrak{a}_{N}^{\prime}+\mathfrak{t}_{N}^{\prime \text { nc }}$. Comme on a

$$
\mathfrak{h}^{\prime \mathrm{nc}}=\mathfrak{a}_{N}^{\prime}+\mathfrak{t}_{N}^{\prime \mathrm{nc}}
$$

on en déduit le point (1).

On considère la racine imaginaire non compacte $\alpha=\left(e_{i_{0}}-e_{j_{0}}\right) c_{N}^{-1}$ de $\mathfrak{h}_{N}^{\prime}$ (avec $i_{0} \in I_{N}^{\prime}$ et $j_{0} \in J_{N}^{\prime}$ ). On remarque que $\alpha$ est aussi une racine imaginaire non compacte de $\mathfrak{h}_{V_{N, c}^{\prime}, \varnothing}^{\prime}=\mathfrak{t}_{N}^{\prime}$. L'égalité (5.2.2) permet d'obtenir

$$
\begin{aligned}
&\langle\partial(w)\left.\operatorname{Chc}(\psi)_{N}\right\rangle_{i_{0}, j_{0}}\left(x^{\prime}\right) \\
& \quad=\mathfrak{C}_{\mathfrak{g}, \mathfrak{g}^{\prime}, N} \mu(N) \varepsilon(N)\left\langle\partial\left(w_{2}\right) \mathbf{C h c}^{U_{N}, V_{N, c}^{\prime}}\left(\partial\left(w_{1}\right) \phi^{G L\left(X_{N}\right)}\left(x_{s}^{\prime}+.\right)\right)_{\varnothing}\right\rangle_{i_{0}, j_{0}}\left(x_{c}^{\prime}\right)
\end{aligned}
$$

où $x^{\prime} \in \mathfrak{h}_{\varnothing}^{\prime}{ }^{\left(i_{0}, j_{0}\right)}, x^{\prime}=x_{s}^{\prime}+x_{c}^{\prime}$ avec $x_{s}^{\prime} \in \mathfrak{h}_{X_{N}}^{\prime}$ et $x_{c}^{\prime} \in \mathfrak{h}_{V_{N, c}^{\prime}, \varnothing}^{\prime}=\mathfrak{t}_{N}^{\prime}$. Si $\operatorname{index}(V)=$ $\operatorname{dim}\left(\mathfrak{a}_{N}^{\prime}\right)$, on a $\operatorname{index}\left(U_{N}\right)=\operatorname{index}(V)-\operatorname{dim}\left(\mathfrak{a}_{N}^{\prime}\right)=0$, ainsi $G\left(U_{N}\right)$ est un groupe compact et d'après le théorème 5.1 .30 , on a

$$
\left\langle\partial\left(w_{2}\right) \mathbf{C h c}^{U_{N}, V_{N, c}^{\prime}}\left(\partial\left(w_{1}\right) \phi^{G L\left(X_{N}\right)}\left(x_{s}^{\prime}+.\right)\right)_{\varnothing}\right\rangle_{i_{0}, j_{0}}=0
$$

On en déduit le résultat dans le cas $\operatorname{index}(V)=\operatorname{dim}\left(\mathfrak{a}_{N}\right)$. Sinon, si $\operatorname{index}(V)>$ $\operatorname{dim}\left(\mathfrak{a}_{N}\right)$, on peut supposer que

$$
\mathrm{A}_{N+\left[i_{0}, j_{0}\right]} \subset I \text { et } \operatorname{im}\left(N+\left[i_{0}, j_{0}\right]\right) \subset J .
$$

On suppose de plus que $w_{2}$ peut s'écrire sous la forme $w_{2}=w_{3} w_{4}$ avec $w_{3}$ indépendant de $x_{i_{0}}^{\prime}$ et $x_{j_{0}}^{\prime}$ et $w_{4} \in \operatorname{Sym}\left(\mathfrak{t}_{N, i_{0}, j_{0}, \mathbb{C}}^{\prime}\right)$ où $\mathfrak{t}_{N, i_{0}, j_{0}}^{\prime}$ désigne le sous-espace de $\mathfrak{t}_{N}^{\prime}$ défini par

$$
\mathfrak{t}_{N, i_{0}, j_{0}}^{\prime}=\left\{x^{\prime} \in \mathfrak{t}_{N}^{\prime} \mid x_{i_{0}}^{\prime}=x_{j_{0}}^{\prime}=0\right\} .
$$

On remarque que

$$
\mathfrak{t}_{N}^{\prime}=\mathfrak{t}_{N, i_{0}, j_{0}}^{\prime} \oplus \mathbb{R i} E_{i_{0}, i_{0}} \oplus \mathbb{R i} E_{j_{0}, j_{0}}
$$

On écrit $x_{c}^{\prime}=x_{1}^{\prime}+x_{2}^{\prime}$ suivant cette décomposition. D'après le théorème 5.1.30, Pour $\theta \in \mathcal{D}\left(\mathfrak{g}^{\prime}\left(V_{N, c}^{\prime}\right)\right)$, on a

$$
\begin{aligned}
& \left\langle\partial\left(w_{2}\right) \mathbf{C h c}^{U_{N}, V_{N, c}^{\prime}}(\theta)_{\varnothing}\right\rangle_{i_{0}, j_{0}}\left(x_{c}^{\prime}\right) \\
& =\mathbf{i} \pi^{2}(-1)^{i_{0}+j_{0}+\mathbf{n}-2 m} 4^{2 \mathrm{n}-4 m-1} \partial\left(w_{3}\right) \mathbf{C h c}^{U_{N, a, b}, V_{N, c, i_{0}, j_{0}}^{\prime}} \\
& \left(\partial\left(c_{a, b} t r_{i_{0}, j_{0}}^{a, b}\left(w_{4}\right)\right)\left(\theta_{\mathfrak{n}_{[a, b]}}^{\left.K_{G\left(U_{N}\right)}\right)}\right)^{G L\left(X_{[a, b]}\right)}\left(x_{2}^{\prime}\right)\right)_{\varnothing}\left(x_{1}^{\prime}\right)
\end{aligned}
$$


où $a$ est un élément de $I_{N}, b$ un élément de $J_{N}, K_{G\left(U_{N}\right)}$ est un sous-groupe compact maximal de $G\left(U_{N}\right)$ et

$$
\begin{aligned}
U_{N, i_{0}, j_{0}} & =\left\{x \in U_{N} \mid x_{i_{0}}=x_{j_{0}}=0\right\} \\
V_{N, c, i_{0}, j_{0}}^{\prime} & =\left\{x \in V_{N, c}^{\prime} \mid x_{i_{0}}=x_{j_{0}}=0\right\} .
\end{aligned}
$$

On a

$$
V_{N, c}^{\prime}=V_{N, c, i_{0}, j_{0}}^{\prime} \oplus \mathbb{R i} e_{i_{0}} \oplus \mathbb{R i} e_{j_{0}}
$$

et

$$
V_{N, c, i_{0}, j_{0}}^{\prime}=V_{N+\left[i_{0}, j_{0}\right], c^{\prime}}^{\prime}
$$

On peut prendre $a=i_{0}$ et $b=j_{0}$ puisque $N+\left[i_{0}, j_{0}\right] \in \Omega$. Il reste à présent à poser

$$
\theta=\partial\left(w_{1}\right) \phi^{G L\left(X_{N}\right)}\left(x_{s}^{\prime}+.\right)
$$

dans l'expression précédente. On remarque que l'on a l'égalité

$$
U_{N, i_{0}, j_{0}}=U_{N+\left[i_{0}, j_{0}\right]}=\left\{x \in V \mid x_{i}=0 \quad \forall i \in \mathrm{A}_{N+\left[i_{0}, j_{0}\right]} \cup \operatorname{im}\left(N+\left[i_{0}, j_{0}\right]\right)\right\} .
$$

On considère un élément $M \in \Omega\left(I_{N+\left[i_{0}, j_{0}\right]}, J_{N+\left[i_{0}, j_{0}\right]}\right)$ et la sous-algèbre de Cartan associée $\mathfrak{h}_{M}$ de $\mathfrak{g}\left(U_{N+\left[i_{0}, j_{0}\right]}\right)$. On observe que $\operatorname{tr}_{i_{0}, j_{0}}^{i_{0}, j_{0}}\left(w_{4}\right)=w_{4}$. On a alors l'égalité suivante grâce au corollaire 5.1.32

$$
\left(\partial\left(c_{i_{0}, j_{0}}\left(w_{4}\right)\right)\left(\theta_{\mathfrak{n}_{\left[i_{0}, j_{0}\right]}}^{K_{G\left(U_{N}\right)}}\right)^{G L\left(X_{\left[i_{0}, j_{0}\right]}\right)}\right)_{M}^{U_{N+\left[i_{0}, j_{0}\right]}}=(-1)^{i_{0}+j_{0}+\mathbf{n}-2 m} \partial\left(c_{i_{0}, j_{0}}\left(w_{4}\right)\right) \theta_{M+\left[i_{0}, j_{0}\right]}^{U_{N}}
$$

Puis, en utilisant le lemme 5.1.31 à deux reprises, on a les égalités

$$
\left(\partial\left(w_{1}\right) \phi^{G L\left(X_{N}\right)}\left(x_{s}^{\prime}+.\right)\right)_{M+\left[i_{0}, j_{0}\right]}^{U_{N}}\left(x_{2}^{\prime}\right)=\mu(N) \varepsilon(N) \partial\left(w_{1}\right) \phi_{M+N+\left[i_{0}+j_{0}\right]}\left(x_{s}^{\prime}+x_{2}^{\prime}\right)
$$

et

$$
\phi_{M+N+\left[i_{0}, j_{0}\right]}=\mu\left(N+\left[i_{0}, j_{0}\right]\right) \varepsilon\left(N+\left[i_{0}, j_{0}\right]\right)\left(\phi^{G L\left(X_{N+\left[i_{0}, j_{0}\right]}\right)}\right)_{M}^{G\left(U_{N+\left[i_{0}, j_{0}\right]}\right)} .
$$

On rappelle la définition de $\mu(N)$ :

$$
\mu(N)=(-1)^{\operatorname{Card}\left\{(i, a) \in I \times \mathbf{A}_{N} \mid i<a\right\}+\operatorname{Card}\left\{(i, a) \in J \times \mathbf{A}_{N} \mid j>N(a)\right\}+\frac{\operatorname{Card}\left(\Lambda_{N}\right)\left(\operatorname{Card}\left(\Lambda_{N}\right)-1\right)}{2}}
$$

On pose $m=\operatorname{Card}\left(\mathbf{A}_{N}\right)$. On obtient les relations suivantes :

$$
\mu\left(N+\left[i_{0}, j_{0}\right]\right) \mu(N)=(-1)^{\operatorname{Card}\left\{i \in I \mid i<i_{0}\right\}+\operatorname{Card}\left\{i \in J \mid j>j_{0}\right\}+m}=(-1)^{i_{0}+j_{0}+\mathbf{n}+m+1} .
$$

et

$$
\mu\left(N+\left[i_{0}, j_{0}\right]\right) \varepsilon\left(N+\left[i_{0}, j_{0}\right]\right) \mu(N) \varepsilon(N)(-1)^{i_{0}+j_{0}+\mathrm{n}}=(-1)^{m} .
$$


Ainsi, on obtient les relations

$$
\begin{aligned}
\left(\partial\left(c_{i_{0}, j_{0}}\left(w_{4}\right)\right)\right. & \left.\left(\theta_{\left.\mathfrak{n}_{\left[i_{0}, j_{0}\right]}\right)}^{K_{G\left(U_{N}\right)}}\right)^{G L\left(X_{\left[i_{0}, j_{0}\right]}\right)}\right)_{M}^{U_{N+\left[i_{0}, j_{0}\right]}} \\
& =\mu(N) \varepsilon(N)(-1)^{i_{0}+j_{0}+\mathrm{n}-2 m} \partial\left(w_{1} c_{i_{0}, j_{0}}\left(w_{4}\right)\right) \phi_{M+N+\left[i_{0}, j_{0}\right]}\left(x_{s}^{\prime}+.\right) \\
& =(-1)^{m} \partial\left(w_{1} c_{i_{0}, j_{0}}\left(w_{4}\right)\right)\left(\phi^{G L\left(X_{N+\left[i_{0}, j_{0}\right]}\right)}\left(x_{s}^{\prime}+.\right)\right)_{M}^{U_{N+\left[i_{0}, j_{0}\right]}} .
\end{aligned}
$$

On en déduit

$$
\begin{aligned}
& \left\langle\partial(w) \operatorname{Chc}(\psi)_{N}\right\rangle_{i_{0}, j_{0}}\left(x^{\prime}\right) \\
& =\mathfrak{C}_{\mathfrak{g}, \mathfrak{g}^{\prime}, N} \delta(N) \varepsilon(N)\left\langle\partial\left(w_{2}\right) \mathbf{C h c}^{U_{N}, V_{N, c}^{\prime}}\left(\partial\left(w_{1}\right) \phi^{G L\left(X_{N}\right)}\left(x_{s}^{\prime}+.\right)\right)\right\rangle_{i_{0}, j_{0}}\left(x_{c}^{\prime}\right) \\
& =A \partial\left(w_{3}\right) \mathbf{C h c}^{U_{N, i_{0}, j_{0}}, V_{N, c, i_{0}, j_{0}}^{\prime}}\left(\partial\left(w_{1}\right) c_{i_{0}, j_{0}}\left(w_{4}\right) \phi^{G L\left(X_{\left.N+i_{0}, j_{0}\right]}\right)}\left(x_{s}^{\prime}+x_{2}^{\prime}+.\right)\right)_{\varnothing}\left(x_{1}^{\prime}\right)
\end{aligned}
$$

où

$$
A=\mathfrak{C}_{\mathfrak{g}, \mathfrak{g}^{\prime}, N} \mu(N) \varepsilon(N) i \pi^{2}(-1)^{i_{0}+j_{0}+\mathrm{n}+m} 4^{2 \mathrm{n}-4 m-1}
$$

Puis, en utilisant l'expression (5.2.2) avec $N+\left[i_{0}, j_{0}\right]$, on obtient

$$
\mathrm{i} \pi^{2} B \partial\left(w_{3} w_{1} c_{i_{0}, j_{0}}\left(w_{4}\right)\right) \mathbf{C h c}(\psi)_{N+\left[i_{0}, j_{0}\right]}\left(x^{\prime}\right)
$$

où

$$
B=(-1)^{i_{0}+j_{0}+\mathrm{n}+m} 4^{2 \mathrm{n}-4 m-1} \frac{\mathfrak{C}_{\mathfrak{g}, \mathfrak{g}^{\prime}, N} \mu(N) \varepsilon(N)}{\mathfrak{C}_{\mathfrak{g}, \mathfrak{g}^{\prime}, N+\left[i_{0}, j_{0}\right]} \mu\left(N+\left[i_{0}, j_{0}\right]\right) \varepsilon\left(N+\left[i_{0}, j_{0}\right]\right)} .
$$

D'après l'égalité (5.2.8), on a

$$
B=4^{2 \mathrm{n}-4 m-1} \frac{\mathfrak{C}_{\mathfrak{g}, \mathfrak{g}^{\prime}, N}}{\mathfrak{C}_{\mathfrak{g}, \mathfrak{g}^{\prime}, N+\left[i_{0}, j_{0}\right]}}
$$

Ainsi, en remarquant que

$$
c_{i, j}(w)=w_{3} w_{1} c_{i, j}\left(w_{4}\right)
$$

on obtient la relation de saut

$$
\begin{aligned}
\left\langle\partial(w) \operatorname{Chc}(\psi)_{N}\right\rangle_{i_{0}, j_{0}} & \left(x^{\prime}\right) \\
= & \mathrm{i} \pi^{2} 4^{2 \mathfrak{n}-4 m-1} \frac{\mathfrak{C}_{\mathfrak{g}, \mathfrak{g}^{\prime}, N}}{\mathfrak{C}_{\mathfrak{g}, \mathfrak{g}^{\prime}, N+\left[i_{0}, j_{0}\right]}} \partial\left(c_{i_{0}, j_{0}}(w)\right) \operatorname{Chc}(\psi)_{N+\left[i_{0}, j_{0}\right]}\left(x^{\prime}\right) .
\end{aligned}
$$

Le point (2) est démontré.

Corollaire 5.2.6. - Pour $\phi \in \mathcal{D}(\mathfrak{g})$ et $N \in \Omega^{\prime}$ tel que $\operatorname{index}(V)>\operatorname{dim}\left(\mathfrak{a}_{N}^{\prime}\right)$, la fonction $\mathbf{C h c}(\phi)_{N}$ vérifie les relations de sauts

$$
\left\langle\partial(w) \mathbf{C h c}(\phi)_{N}\left(x^{\prime}\right)\right\rangle_{i_{0}, j_{0}}\left(x^{\prime}\right)=\mathrm{i} \partial\left(c_{i_{0}, j_{0}}(w)\right) \mathbf{C h c}(\phi)_{N+\left[i_{0}, j_{0}\right]}\left(x^{\prime}\right)
$$

pour $w \in \operatorname{Sym}\left(\mathfrak{h}_{\mathbb{C}}^{\prime}\right)$ si et seulement si

$$
\mathfrak{C}_{\mathfrak{g}, \mathfrak{g}^{\prime}, N+\left[i_{0}, j_{0}\right]}=\pi^{2} 4^{2 \mathfrak{n}-4 m-1} \mathfrak{C}_{\mathfrak{g}, \mathfrak{g}^{\prime}, N}
$$


Remarque. - Pour $N \in \Omega$, on considère une fonction polynomiale de $\operatorname{Sym}\left(\mathfrak{h}_{N, \mathbb{C}}^{\prime}\right)$ telle que $\operatorname{deg}_{x_{i}^{\prime}}(P) \leqslant 1$ pour $i \in I_{N}^{\prime} \cup J_{N}^{\prime}$ alors on peut montrer que la fonction

$$
x^{\prime} \longmapsto P\left(x^{\prime}\right) \operatorname{Chc}(\phi)_{N}\left(x^{\prime}\right)
$$

est bornée $\operatorname{sur} \mathfrak{h}_{N}^{\prime}$ 



\section{BIBLIOGRAPHIE}

[1] F. Bernon - «Propriétés de l'intégrale de Cauchy Harish-Chandra pour certaines paires duales d'algèbres de Lie», C. R. Acad. Sci. Paris Sér. I Math. 334 (2002), p. 945-948.

[2] A. Bouaziz - «Intégrales orbitales sur les algèbres de Lie réductives », Invent. Math. 115 (1994), p. 163-207.

[3] L. Hormander - The Analysis of Linear Partial Differential Operator I, Springer-Verlag, 1983.

[4] R. Howe - «Transcending Classical Invariant Theory », J. Amer. Math. Soc. 2 (1989), p. 535-552.

[5] C. Moeglin, M.-F. Vignéras \& J.-L. Waldspurger - Correspondance de Howe sur un corps p-adique, Lect. Notes in Math., vol. 1291, Springer-Verlag, 1987.

[6] T. Przebinda - «A Cauchy Harish-Chandra Integral, for a real reductive dual pair», Invent. Math. 141 (2000), p. 299-363.

[7] W. Schmid - « On the Character of the Discrete Series. The Hermitian Symmetric Case», Invent. Math. 30 (1975), p. 47-144.

[8] M. Sigiura - « Conjugate classes of Cartan subalgebras in real semisimple Lie algebras », J. Math. Soc. Japan 11 (1959), p. 374-434.

[9] J.-C. Tougeron - Idéaux de fonctions différentiables, Springer-Verlag, 1972.

[10] F. Treves - Topological Vector Spaces, Distributions and Kernels, Academic Press, 1967.

[11] V.S. VARADARAJAN - Harmonic Analysis on Real Reductive Groups, Lect. Notes in Math., vol. 576, Springer-Verlag, 1977. 